\title{
Polymer Confined Self Assembly
}

\author{
By \\ Molla Rafiquel Islam \\ BSc (Hons.), MSc.
}

A thesis submitted to the Faculty of Graduate and Postdoctoral Affairs

in partial fulfillment of the requirements for the degree of

\section{Doctor of Philosophy}

In Chemistry

Carleton University

Ottawa, Ontario

February, 2011

(C) 2011, Molla Rafiquel Islam 
Library and Archives

Canada

Published Heritage

Branch

395 Wellington Street

Ottawa ON K1A ON4

Canada
Bibliotheqque et

Archives Canada

Direction du

Patrimoine de l'édition

395 , rue Wellington

Ottawa ON K1A ON4

Canada
Your file Votre référence

ISBN: 978-0-494-81575-5

Our file Notre référence

ISBN: $978-0-494-81575-5$
NOTICE:

The author has granted a nonexclusive license allowing Library and Archives Canada to reproduce, publish, archive, preserve, conserve, communicate to the public by telecommunication or on the Internet, loan, distribute and sell theses worldwide, for commercial or noncommercial purposes, in microform, paper, electronic and/or any other formats.

The author retains copyright ownership and moral rights in this thesis. Neither the thesis nor substantial extracts from it may be printed or otherwise reproduced without the author's permission.
AVIS:

L'auteur a accordé une licence non exclusive permettant à la Bibliothèque et Archives Canada de reproduire, publier, archiver, sauvegarder, conserver, transmettre au public par télécommunication ou par l'Internet, prêter, distribuer et vendre des thèses partout dans le monde, à des fins commerciales ou autres, sur support microforme, papier, électronique et/ou autres formats.

L'auteur conserve la propriété du droit d'auteur et des droits moraux qui protège cette thèse. $\mathrm{Ni}$ la thèse ni des extraits substantiels de celle-ci ne doivent être imprimés ou autrement reproduits sans son autorisation.
In compliance with the Canadian Privacy Act some supporting forms may have been removed from this thesis.

While these forms may be included in the document page count, their removal does not represent any loss of content from the thesis.
Conformément à la loi canadienne sur la protection de la vie privée, quelques formulaires secondaires ont été enlevés de cette thèse.

Bien que ces formulaires aient inclus dans la pagination, il n'y aura aucun contenu manquant.

\section{Canadä}


The undersigned hereby recommend to the faculty of Graduate Studies and Research

For the acceptance of the thesis

\section{Polymer Confined Self Assembly}

Submitted by

\section{Molla Rafiquel Islam}

in partial fulfillment of the requirements for the degree of

Doctor of Philosophy

Christopher J. Barrett, External Examiner

Dr. P. R. Sundararajan, Thesis Supervisor

Dr. Bob Burk, Chair, Department of Chemistry

Carleton University

February, 2011 


\section{ABSTRACT}

Self assembly is ubiquitous in nature and design of synthetic systems by imparting the self assembly codes is currently an active area of research. In this thesis, we study the effect of chemically or physically confining a self-assembling molecule on its association in solution and morphology in the solid state. For the case of chemical confinement, the self assembly and morphology of amphiphilic perylene diimides have been studied by covalently attaching hydrophilic Jeffamine ${ }^{(3)}$ (a polyether amine) to the imide nitrogen of the perylene diimide. on either on side or on both sides. In the first case, the molecule is a pseudo block copolymer of the rod-coil type. It can also be considered a macromolecular inverse surfactant, since the "head group" is a water insoluble aromatic segment, and the tail (Jeffamine ${ }^{(3)}$ ) is hydrophilic. When the Jeffamine ${ }^{(8)}$ is attached to both sides of the perylene diimide, it is a Gemini surfactant. We also designed a molecule with Jeffamine ${ }^{(B)}$ attached to one side of the perylene diimide and a short alkyl chain to the other end. While attaching a hydrophilic group gives aqueous based self assembly, the bydrophobic group enables self assembly in aqueous solvent mixtures. We have shown that the mode of aggregation and morphology are different for these diimides and the extent of aggregation can be controlled by changing the concentration and solvent mixtures. The aggregates of diimides show high thermal and $\mathrm{pH}$ stability and, due to their fluorescent properties these water soluble perylene diimides may find application in biological probes. We have also studied the change in morphology from bundles of needles to hollow tubes and stability of amphiphilic perylene diimide as a function of the length of hydrophobic alkyl group. Nonaqueous based self assembly is also important for applications in optoelectronic devices. lasers and molecular sensors etc. where perylene diimide shows high fluorescent intensity 
but low tendency to aggregate. In this respect we have studied the of perylene diimide with polystyrene attached to both sides in non-aqueous solvents. We have characterized the morphology, extent of aggregation and stability. This diimide shows spherical or web like morphologies from nano to micrometer size.

For the case of physical confinement. we studied the self assembly of a phthalocyanine ( $\pi$ interaction mediated self assembly) and a series of biscarbamates (hydrogen bond enabled self assembly) dispersed in polymer matrices. Depending on the casting solvent. the morphology of phthalocyanine dispersed in polycarbonate and PMMA matrix has either tubular or subsurface droplet like morphology. In the case of biscarbamates with two $\mathrm{H}$ - bonding moieties, the morphology depends on the side chain length. A donut-like morphology forming a meandering chain for short chain biscarbamates and needles for long side chain biscarbamates in polycarbonate was seen, which is dependent on concentration and thermal treatment. Normally, when a small molecule phase separates from a polymer matrix, it diffuses to the surface and crystallizes. However, in the case of a self assembling molecule, when the association due to self assembly is faster than the diffusion to the surface, we see sub-surface aggregation.

This study enables us to control the morphology of photo-functional molecule with $\pi$-bonding moiety or small organic molecule with $\mathrm{H}$-bonding moiety in a polymer confined state in solution and solid state and hence paves the way for controlling the properties to get enhanced or new applications. 


\section{ACKNOWLEDGEMENTS}

I am very glad to have had an opportunity to do Ph.D. in Chemistry under the supervision of Professor P. R. Sundararajan. During the last few years, he has grown me as a researcher. I was impressed by his thought and guidance throughout my research. He supported me immensely. I am grateful to almighty Allah for helping me to complete my PhD.

I would like to thank Mohammad Mostofa Kamal Khan and Dr. Sundar Saimani for their help during my initial set up in the lab. I would be happy to name Elianne Dahan, Anita Chan and all other visiting scholars and post docs worked here during the last few years. I would like to give special thanks to the Shah Jalal university of Science and Technology authority for granting me a study leave. NSERC for financial support and chemistry department, organizations or persons for various scholarships (Molecular Recognition and Inclusion Scholarship, Emmett Dunne Scholarship in Chemistry. International Tuition Scholarship for Academic Excellence etc.) during my $\mathrm{PhD}$.

I do mention the desire of my parents for their youngest son to be a chemist from my childhood which actually guided and inspired me to take chemistry as my major.

Last but not the least I would like to thank my wife Shammi for her support and understanding the difficulty of sharing time in the lab and with family and my daughter Farishta for her smile that helps me always to reduce the stress of doing research. and organizing all of them as a thesis at last. 


\section{TABLE OF CONTENTS}

Abstract

iii

Acknowledgements

v

Table of Contents

vi

List of Schemes, Tables and Figures

xiv

List of Abbreviations

xxviii

List of Appendices

$\mathrm{xxx}$

\section{Chapter I: Introduction}

$\begin{array}{lll}1.1 & \text { Self assembly } & 2\end{array}$

1.2 Current Research on Self Assembly and Morphology 4

1.3 Self Assembly in a Confined State 5

1.4 Materials Used and Their Properties $\quad 7$

1.4.1 Perylene and Perylene Bisimides 7

1.4.1.1 Synthesis of Perylene Diimides 9

1.4.1.2 Crystal Structure and Packing $\quad 12$

1.4.1.2.1 Perylene Diimide Aggregation in Solid State 12

1.4.1.2.2 Perylene Diimide Aggregation in Solution 13

1.4.1.2.3 Optical, Electrochemical and Liquid Crystalline $\begin{array}{ll}\text { Properties of Perylene Diimides } & 13\end{array}$

1.4.2 1,4,8.11,15.18,22.25-Octabutoxy-29H,31H-phthalocyanine 


\section{TABLE OF CONTENTS Continued}

$\begin{array}{ll}\text { 1.4.3 Biscarbamates } & 16\end{array}$

1.5 Concepts and Terms Used in Self Assembly 17

1.5.1 Electronic Energy Level, Absorption and Emission 17

1.5.2 Definition of H and J Aggregates: Mode of aggregation 20

1.5.2.1 Pattern of Aggregates in the Solution 20

$\begin{array}{ll}\text { 1.5.2.2 Absorption Spectrum of Aggregates } & 21\end{array}$

$\begin{array}{ll}1.5 .3 \text { Theory } & 22\end{array}$

1.5.3.1 The Exciton Theory 22

1.5.3.2 The Nature and Origin of $\pi-\pi$ Interaction 23

$\begin{array}{ll}\text { 1.6 Thesis Objective } & 25\end{array}$

$\begin{array}{ll}\text { 1.7 Thesis Outline } & 26\end{array}$

$\begin{array}{ll}1.8 \text { References } & 30\end{array}$

\section{Chapter II: Experimental}

$\begin{array}{ll}2.1 \text { Introduction } & 41\end{array}$

2.2 Instruments and Methods $\quad 41$

2.2.1 FTIR Spectroscopy $\quad 41$

2.2.2 UV-Visible Spectroscopy 43

2.2.3 Fluorescent Spectroscopy $\quad 45$

$\begin{array}{ll}\text { 2.2.4 NMR Spectroscopy } & 47\end{array}$

2.2.5 Differential Scanning Calorimetry, DSC 48 
TABLE OF CONTENTS Continued

2.2.6 Polarizing Optical Microscopy, OM $\quad 50$

2.2.7 Scanning Electron Microscopy, SEM 52

2.2.8 Dynamic Light Scattering, DLS 53

2.2.9 X-ray Diffraction, XRD 55

$\begin{array}{ll}2.4 \text { References } & 59\end{array}$

Chapter III: Self Assembly of Rod-Coil and Coil-Rod-Coil Amphiphilic Polymers Based on Perylene Tetracarboxylic Diimide in Water, Mixed Solvents and in Bulk $\quad 61$

$\begin{array}{ll}3.1 \text { Introduction } & 62\end{array}$

$\begin{array}{ll}3.2 \text { Experimental Section } & 65\end{array}$

3.2.1 Synthesis of N-isobutyl-3, 4, 9, 10-perylenetetracarboxylic $\begin{array}{ll}\text { monoanhydride monoimide } & 67\end{array}$

3.2.2 Synthesis of MJ-PTCDI $\quad 68$

3.2.3 Synthesis of DJ PTCDI $\quad 69$

$\begin{array}{ll}3.3 \text { Results and Discussion } & 70\end{array}$

3.3.1 UV-Vis and Fluorescence Spectroscopy $\quad 70$

$\begin{array}{ll}\text { 3.3.2 Effect of Solvent } & 77\end{array}$

3.3.3 Aggregate Stability $\quad 86$

$\begin{array}{ll}3.3 .4 \text { Microscopy } & 95\end{array}$

$\begin{array}{ll}\text { 3.3.5 Thermal Analysis } & 104\end{array}$ 


\section{TABLE OF CONTENTS Continued}

3.3.6 X-Ray Analysis 106

$\begin{array}{ll}3.4 \text { Conclusions } & 108\end{array}$

$\begin{array}{ll}3.5 \text { References } & 109\end{array}$

Chapter IV: Tuning the Morphology of Amphiphilic Pseudo Block

Copolymer of Perylene Diimide From Folded Sheet to Tube by Modulating

the Hydrophobic Chain Length 113

4.1 Introduction $\quad 114$

4.2 Experimental 116

4.2.1 Synthesis of Perylene Monoimide Monoanhydride $\quad 118$

4.2.2 Synthesis of Asymmetric Perylene Diimide $\quad 120$

$\begin{array}{ll}4.3 \text { Results and Discussion } & 121\end{array}$

$\begin{array}{lr}\text { 4.3.1 UV-Vis and Fluorescent Spectroscopy } & 121\end{array}$

$\begin{array}{ll}\text { 4.3.2 Effect of Polarity } & 133\end{array}$

$\begin{array}{ll}\text { 4.3.3 Effect of Temperature } & 137\end{array}$

$\begin{array}{ll}\text { 4.3.4 Microscopy } & 140\end{array}$

4.3.5 Thermal Analysis $\quad 151$

$\begin{array}{ll}\text { 4.3.6 XRD Analysis } & 152\end{array}$

$\begin{array}{ll}4.4 \text { Conclusion } & 155\end{array}$

$\begin{array}{lr}4.5 \text { References } & 157\end{array}$ 
TABLE OF CONTENTS Continued

Chapter V: Self assembly of Perylene Bisimides Jacketed by Polystyrene

5.1 Introduction

5.2 Experimental

5.2.1 Synthesis of $N, N$ '-polystyrene -3, 4, 9, 10-perylene

tetracarboxylic diimide

5.3 Results and Discussion

5.3.1 Self assembly in Solution Studied by Absorption and Fluorescence Spectroscopy

5.3.2 Effect of Polarity on Self Assembly

176

5.3.3 Effect of Temperature

Chapter VI: Tubular or Sub-Surface Morphology of a Substituted

Phthalocyanine Upon Self Assembly in Polymer Matrices: 


\section{TABLE OF CONTENTS Continued}

$\begin{array}{ll}6.1 \text { Introduction } & 195\end{array}$

$\begin{array}{ll}6.2 \text { Experimental } & 198\end{array}$

$\begin{array}{ll}6.3 \text { Results and Discussion } & 200\end{array}$

$\begin{array}{ll}\text { 6.3.1 Microscopy } & 201\end{array}$

$\begin{array}{ll}\text { 6.3.2 Film Thickness } & 215\end{array}$

$\begin{array}{ll}\text { 6.3.3 NMR Spectra } & 217\end{array}$

$\begin{array}{ll}\text { 6.3.4 UV-Vis Spectra } & 218\end{array}$

$\begin{array}{ll}\text { 6.3.5 X-ray Diffraction } & 224\end{array}$

6.3.6 Viscosity of the Solutions and Growth Morphology 230

$\begin{array}{ll}6.4 \text { Conclusions } & 231\end{array}$

$\begin{array}{ll}6.5 \text { References } & 233\end{array}$

Chapter VII: Morphology of a Hydrogen Bond Mediated Self-Assembling

Small Molecule in a Polycarbonate Matrix 236

$\begin{array}{ll}7.1 \text { Introduction } & 237\end{array}$

$\begin{array}{ll}7.2 \text { Experimental } & 239\end{array}$

$\begin{array}{ll}\text { 7.2.1 Materials } & 239\end{array}$ 
TABLE OF CONTENTS Continued

$\begin{array}{ll}\text { 7.2.2 Making of Films } & 239\end{array}$

$\begin{array}{ll}\text { 7.2.3 Characterization Methods } & 240\end{array}$

$\begin{array}{ll}7.3 \text { Results and Discussion } & 241\end{array}$

$\begin{array}{ll}\text { 7.3.1 Polarizing Optical Microscopy } & 241\end{array}$

$\begin{array}{ll}\text { 7.3.2 Scanning Electron Microscopy } & 247\end{array}$

$\begin{array}{ll}\text { 7.3.3 Thermal Reversibility } & 248\end{array}$

$\begin{array}{ll}7.4 \text { Conclusions } & 251\end{array}$

$\begin{array}{ll}7.5 \text { References } & 253\end{array}$

Chapter VIII: Conclusions and Recommendations for Future Work. 255

$\begin{array}{ll}8.1 \text { Conclusions } & 256\end{array}$

8.2 Recommendations for Future Work $\quad 260$ 
TABLE OF CONTENTS Continued

Appendix A: FTIR spectra.

Appendix B: NMR spectra.

Appendix C: Photographs of solutions. 


\section{List of Schemes, Tables and Figures}

Scheme 1.1: Synthesis of symmetrical perylene diimides.

Scheme 1.2: Non-symmetrically substituted perylene diimide precursor.

Scheme 1.3: Synthesis of asymmetric diimide.

Figure 1.1: a) SEM images of Nano-fiber ${ }^{5 a}$ and b) Nano-sphere morphologies from amphiphilic perylene diimides c) SEM images showing the morphogenesis of helical patterns, from the first-order unclustered epoxy nanobristle to the fourth-order coiled bundle. ${ }^{\text {10a }}$ d) Assembly of dipeptide-naphthalene diimide into left-handed fibers (top) and ribbons (Bottom). ${ }^{12 \mathrm{e}}$

Figure 1.2: Schematic of comparison of block copolymer approach and chemical confinement approach without covalent bond (upper) and confined by covalent bond (lower).

Figure 1.3: Schematic of polymer confined state. A chromophore is (a) confined in polymer matrix by covalent bond and (b) confined without covalent bond.

Figure 1.4: Perylene diimide skeleton highlighting bay and imide positions.

Figure1.5: Projection of molecular model of 1,4,8,11.15,18.22,25-Octabutoxy- 


\section{List of Schemes, Tables and Figures continued}

29H,31H-phthalocyanine drawn by Accelrys MatStudio ver 3.0. Oxygen

and nitrogen atoms are marked with red and blue respectively.

Figure 1.6: Chemical structure of Biscarbamate. Here $R=C_{4}$ to $C_{18}$

Figure 1.7: Schematic of Electronic energy levels.

Figure 1.8: Schematic of absorption and emission process and corresponding

spectra.

Figure 1.9: One dimensional assembly in solution by planar molecule.

Figure 1.10: $\mathbf{H}$ - and $\mathbf{J}$ - type stacking, geometry and absorption pattern.

Figure 2.1: Energy levels.

Figure 2.2: Jablonski diagram.

Figure 2.3: Schematic representation of Differential Scanning Calorimeter.

Figure 2.4: A typical DSC plot.

Figure 2.5: Principle of diffraction. Figure shows a plane of beam of parallel xrays penetrating a stack of planes of spacing $d$, at a glancing angle of incidence $\theta$. Each plane must be pictured as reflecting a fraction of the incident beam. ${ }^{9}$

Figure 2.6: Schematic of the Bragg diffraction with the powder X-ray diffractometer used for the present study [redrawn from Alexander $\left.{ }^{17}\right]$. Here, 


\section{List of Schemes, Tables and Figures continued}

$S_{1}, S_{2}$ and $S_{3}$ are the divergence slit, receiving slit and scatter slit respectively.

Scheme 3.1: Synthesis of DJ-PTCDI and MJ-PTCDI. ${ }^{23}$

Figure 3.1: UV absorption spectra of (a) DJ-PTCDI and (b) MJ-PTCDI at increasing concentrations at the direction of the arrow from $1 \times 10^{-7}$ to $9 \times 10^{-5}$

M.

Figure 3.2: Fluorescence spectra of DJ-PTCDI in water with different

concentrations.

Figure 3.3: Fluorescence spectra of MJ-PTCDI in water with different concentrations.

Figure 3.4: Absorption spectra of (a) DJPTCDI and (b) MJPTCDI in different solvents at $10^{-4} \mathrm{M}$; (c): in solid state from drop-cast films.

Figure 3.5: UV Visible spectra of (a) DJ-PTCDI and MJ-PTCDI (b) in solvent $\mathrm{CH}_{2} \mathrm{Cl}_{2}$ : Hexane mixtures at $10^{-5} \mathrm{M}$.

Figure 3.6: UV-Vis absorption spectra of (a) DJ-PTCDI and

(b) MJ-PTCDI in Acetone/Water mixture at $10^{-5} \mathrm{M}$.

Figure 3.7: Emission spectra of (a) DJ-PTCDI and (b) MJ-PTCDI

in acetone-water binary mixtures at $10^{-5} \mathrm{M}$. 


\section{List of Schemes, Tables and Figures continued}

Figure 3.8: Mole fraction of aggregates $\left(\alpha_{\mathrm{agg}}\right)$ as a function of solvent

composition (water/acetone) for MJ-PTCDI and DJ-PTCDI.

Figure 3.9: Absorption spectra of (a) DJPTCDI and (b) MJPTCDI at different $\mathrm{pH}$ at $10^{-5} \mathrm{M}$.

Figure 3.10: Variable temperature UV-Vis spectra of (a) DJ-PTCDI and

(b) MJ-PTCDI in water and in acetone-water (1:9) mixture (c and d respectively).

Figure 3.11: Mole fraction of aggregates vs temperature curve for MJ-PTCDI and DJ-PTCDI in acetone-water (1:9) mixture.

Figure 3.12: ${ }^{1} \mathrm{HNMR}$ spectra of (a) DJ-PTCDI and (b) MJ-PTCDI in $\mathrm{CDCl}_{3}$

and at various temperatures in $\mathrm{D}_{2} \mathrm{O}$.

Figure 3.13: (a) DJ-PTCDI as is (b) MJ-PTCDI as is (c), DJ-PTCDI drop-cast

(d) MJ-PTCDI drop-cast (e) SEM of DJ-PTCDI freeze-dried and

(f) SEM of MJ-PTCDI freeze-dried. Here a, b. c, and d are of same scale.

Figure 3.14: Log normal distribution of the DLS data of (a) DJPTCDI and

(b) MJPTCDI in water at $10^{-4} \mathrm{M}$ concentration.

Figure 3.15: SEM and OM of drop-cast films of aged aqueous solutions 


\section{List of Schemes, Tables and Figures continued}

of DJ-PTCDI ( $a$ and $c$ ) and MJ-PTCDI ( $b$ and $d)$. OM images $c$ and $d$ are

of same scale. An enlargement of a part of (d) is shown in (e).

Figure 3.16: SEM Images of drop-cast films of (a) DIPTCDI and (b) MJPTCDI from an aged $\mathrm{pH}=6$ solution.

Figure 3.17: Optical and Scanning electron micrographs of drop-cast solutions

of DJ-PTCDI from various solvents. All the OM images are of same scale.

Figure 3.18: Optical and Scanning electron micrographs of drop-cast solutions

of MJ-PTCDI from various solvents. All the OM images are of same scale.

Figure 3.19: DSC of DJ-PTCDI (a) and MJ-PTCDI (b).

Figure 3.20: X-ray diffraction profile of (a) DJ-PTCDI and (b)MJ-PTCDI.

Scheme 4.1: Synthesis of monoimide monoanhydride from PTDA.

Scheme 4.2: Synthesis of asymmetric perylene diimide.

Table 4.1: $d$-spacing values from X-ray profile of PJ-PTCDI, HJ-PTCDI and

OJ-PTCDI. Here strong, medium and weak reflections are marked as

$s, m$, and w respectively.

Figure 4.1: The UV-Vis of (a) PJ-PTCDI, (b) HJ-PTCDI and (c) OJ-PTCDI in different solvents at $1 \times 10^{-5} \mathrm{M}$. 


\section{List of Schemes, Tables and Figures continued}

Figure 4.2: The UV-Vis spectra of (a) PJ-PTCDI,

(b) HJ-PTCDI and (c) OJ-PTCDI in water from $1 \times 10^{-7}-1 \times 10^{-4} \mathrm{M}$.

Figure 4.3: $\mathrm{H}$ and $\mathrm{J}$ - stacking.

Figure 4.4: Fluorescent spectra of (a) PJPTCDI and (b) H.JPTCDI

in aqueous solution.

Figure 4.5: Molecular model of the perylene diimides PJ-PTCDI,

HJ-PTCDI and OJ-PTCDI drawn by Hyperchem 7.

Figure 4.6: (a) UV-Vis and (b) Fluorescent spectra of PJ-PTCDI in acetone-water mixture at $1 \times 10^{-5} \mathrm{M}$.

Figure 4.7: (a) UV-Vis and (b) fluorescent spectra of PJ-PTCDI in methanol-water mixture at $1 \times 10^{-5} \mathrm{M}$.

Figure 4.8: Temperature dependent UV-Vis spectra of MJ-PTCDI (a) water,

(b) acetone-water (c) methanol-water (c) and (d) HJ-PTCDI in water at $10^{-5}$

M.

Figure 4.9: The optical micrographs of (a) PJ-PTCDI, (b) H.I-PTCDI

and (c) OJ-PTCDI respectively.

Figure 4.10: The SEM of as such (a) PJ-PTCDI, (b) HJ-PTCDI and 


\section{List of Schemes, Tables and Figures continued}

(c) OJ-PTCDI

Figure 4.11: The SEM of PJ-PTCDI (a), HJ-PTCDI (b) and

OJ-PTCDI ( $c, d$, e, and f) respectively in water solution. The samples

were prepared by dropping the solutions on the SEM glass slides and

then dried by ambient condition. Here $d$, e and f images were taken

by tilting the slide of the sample by $47^{\circ}$ to see the tube nature of the

packing from OJ-PTCDI.

Figure 4.12: SEM images of slowly formed self assembled structures

of different diimides from $10^{-5} \mathrm{M}$ aqueous solution.

Figure 4.13: SEM images of PJPTCDI (a), HJPTCDI (b) and

(c) OJPTCDI self assembled from different solvents.

Figure 4.14: The DSC of (a) PJ-PTCDI, (b) HJ-PTCDI and (c) OJ-PTCDI .

Figure 4.15: a) The XRD of (a) PJ-PTCDI, (b) HJ-PTCDI and (c) OJ-PTCDI.

The XRD patterns were recorded by taking the pure compounds

on the glass slides.

Scheme 5.1: Reaction of amino terminated polystyrene with perylene

dianhydride to give coil-rod-coil block copolymer, PS-b-Perylene-b-PS. 


\section{List of Schemes, Tables and Figures continued}

Scheme 5.2: (a) $\mathrm{C}-\mathrm{H}^{\cdots} \pi$ interaction between a phenyl group of a polystyrene segment and the PTCDI of an adjacent molecule in a good solvent for polystyrene (b) $\pi$ stacking between neighboring PTCDI segments when the polystyrene segment is coiled (collapsed).

Scheme 5.3: Schematic of the formation of vesicles of PS-PTCDI-PS.

Figure 5.1: UV-Vis and fluorescent spectra of PS-PTCDI-PS in different solvents at $10^{-4} \mathrm{M}$. The inset of each figure shows the solution without and with excitation at $366 \mathrm{~nm}$.

Figure 5.2: UV-Vis and fluorescent spectra of PS-PTCDI-PS in chloroform from $10^{-7}$ to $10^{-4} \mathrm{M}$.

Figure 5.3: (a) UV-Vis spectra of PS-PTCDI-PS drop cast and dried on glass slides from different solvents at $10^{-4} \mathrm{M}$. (b) Absorption spectra of such films from mixtures of solvents. (c) photographs of the glass slides with PS-PTCDIPS from different solvents without excitation and (d) excited at $365 \mathrm{~nm}$.

Figure 5.4: Proposed model of (a) $\mathrm{C}-\mathrm{H}^{*} \pi$ interaction between a phenyl group of a polystyrene segment and the PTCDI of an adjacent molecule in a good solvent for polystyrene. (b) $\pi$-stacking between neighboring PTCDI 


\section{List of Schemes, Tables and Figures continued}

segments when the polystyrene segment is coiled (collapsed).

Figure 5.5: (a) UV-Vis and (b) fluorescent spectra of PSPTCDIPS in

Chloroform-Hexane mixtures at $10^{-5} \mathrm{M}$. The inset of both of the figures shows the solutions with different chloroform and hexane ratio without and with

excitation at $365 \mathrm{~nm}$.

Figure 5.6: (a) UV-Vis and (b) fluorescent spectra of PSPTCDIPS in chloroform-methanol mixtures at $10^{-5} \mathrm{M}$.

Figure 5.7: (a) UV-Vis spectra of PS-PTCDI-PS in Acetone-water mixtures at $10^{-5} \mathrm{M}$. Photographs of solutions before (b) and (c) after excitation, respectively.

Figure 5.8: UV-Vis spectra of PS-PTCDI-PS in acetone--methanol mixtures $\left(10^{-5} \mathrm{M}\right)$

Figure 5.9: Temperature dependent UV-Vis spectra of PS-PTCDI-PS in Chloroform-Methanol : 1/9 and Acetone-Water system : $1 / 9$ system at $10^{-5} \mathrm{M}$.

Figure 5.10: Optical micrographs of PS-PTCDI-PS in drop cast films from different solvents. The scale bar is the same for all the images.

Figure 5.11: SEM micrographs of PS-PTCDI-PS drop cast films from different 


\section{List of Schemes, Tables and Figures continued}

solvents. $(a, b)$ : acetone; $(c, d)$ : chloroform; $(e, f)$ : toluene; $(g)$ : cyclohexane;

(h) hexane

Figure 5.12: Schematic of the formation of vesicles of PS-PTCDI-PS.

Figure 5.13: SEM micrographs of PS-PTCDI-PS drop cast films from

chloroform/hexane and THF/ water mixtures.

Figure 5.14: X-ray diffraction traces of PS-PTCDI-PS and PTDA.

Figure 6.1: Chemical structure of Pc, BPAPC and PMMA.

Figure 6.2: Optical micrographs of Pc/BPAPC/TCE films with (a-e):

0.85 to $5 \%$ concentration of Pc. The scale is the same for (a-e).

Figure 6.3: a) Average length of the crystals in films with different

wt $\%$ of Phthalocyanine b) Variation of the length of the crystals with time

in situ in films made with tetrachloroethane with different concentrations of

phthalocyanine in BPAPC.

Figure 6.4: SEM of Pc/BPAPC/TCE films with (a-e): $0.85-5 \%$ concentrations

of Pc. A higher magnification image of 5/Pc/BPAPC/TCE is shown in (f).

Figure 6.5: Tube formation of Phthalocyanine.

Figure 6.6: Projection of the phthalocyanine molecule after energy 


\section{List of Schemes, Tables and Figures continued}

minimization (Accelrys Materials Studio ver 3.0) showing the

non-planarity of the molecule with octabutoxy substituents.

208

Figure 6.7: Crystals of phthalocyanine that slowly formed from chloroform

and TCE solutions. (a) Saturated solutions were prepared to determine

the molar solubility. Over time (weeks) crystals formed on the walls of the

bottles, as above. SEM images of the crystals that formed inside the vial

from TCE (b) and from chloroform (c) are shown.

Figure 6.8: Optical micrographs of Pc/BPAPC/Cl films (a-e): $0.85-5 \%$.

The scale bar is the same for a-e. Confocal microscopic image of

5PcBPAPCCHL is also given ( $\mathrm{f})$.

Figure 6.9: $\mathrm{SEM}$ of $\mathrm{Pc} / \mathrm{BPAPC} / \mathrm{Cl}$ films (a-c): $3-5 \%(w t)$.

Figure 6.10: Optical micrographs of Pc/PMMA/TCE films with (a-e): 0.85 to

$5 \%$ concentration of $\mathrm{Pc}$. The scale bar is the same for a-e. Figure $\mathrm{f}$ and $\mathrm{g}$ are

bright and dark field OM images of 5Pc/PMMA/TCE films respectively to

highlight the tube formation (marked with arrow).

Figure 6.11: SEM of 5Pc/PMMA/TCE.

Figure 6.12: Optical micrographs of $5 \mathrm{Pc} / \mathrm{BPAPC} / \mathrm{Cl}$ (top) and 


\section{List of Schemes, Tables and Figures continued}

5Pc/BPAPC/TCE films prepared with spin coater at two different speeds.

The scale bar is same for all.

Figure 6.13: ' $\mathrm{H}$ NMR of Phthalocyanine in TCE and Chloroform.

Figure 6.14: ${ }^{13} \mathrm{CNMR}$ of Phthalocyanine in TCE and Chloroform.

Figure 6.15: UV-Vis spectra of Pc in (a) TCE and (b) chloroform;

(c) films of Pc/BPAPC/TCE and (d) Pc/PMMA/TCE with increasing $\mathrm{c}$

oncentration of Pc. (e) and (f) show the UV-Vis spectra of Pc/BPAPC/ Cl

and $\mathrm{Pc} / \mathrm{PMMA} / \mathrm{Cl}$ films respectively with $1 \%$ of $\mathrm{Pc}$ only. In this case,

the intensity goes beyond the scale as the concentration increases.

Figure 6.16: Photograph of PCBPAPC solution in TCE and Chloroform

Figure 6.17: Photograph of Pc solution in different solvents after exciting at $366 \mathrm{~nm}$. Pc dissolved with high concentration doesn't show fluorescence.

High concentration in TCE refers to $1 \mathrm{mg} / \mathrm{mL}$ and low concentration

denotes $0.01 \mathrm{mg} / \mathrm{mL}$.

Figure 6.18: The color change of Pc dissolved in TCE (concentration increases in the direction of the arrow). The lower photograph was taken by exciting the solutions at $366 \mathrm{~nm}$. As we lower the concentration the solution in 


\section{List of Schemes, Tables and Figures continued}

TCE starts fluorescing and with very low concentration fluorescent intensity goes down. For all the other solvents we don`t see either any color change or fluorescence with concentration.

Figure 6.19: X-Ray powder diffraction profiles of (a) Pc as received (b) Pc crystallized from TCE and (c) from chloroform. (b) and (c) are from the bottles shown in Figure 5.

Figure 6.20: X-Ray powder diffraction profiles of (a) 5PcBPAPCTCE and 5Pc/PMMA/TCE and (b) 5Pc/BPAPC/CHL and 5Pc/PMMA/CHL films.

Figure 7.1: The structures of simple and biscarbamates.

Figure 7.2: Optical micrographs of polycarbonate films

containing (3 wt \%) biscarbamates with different alkyl chain lengths.

Figure 7.3: Optical micrographs of films that were dried at $50^{\circ} \mathrm{C}$.

Figure 7.4: Optical micrographs of polycarbonate films with different concentrations of $\mathrm{C}_{4}$ : (a) $0.75 \%$ (b) $2 \%$ (c) $4 \%$ (d) $6 \%$ (e) $8 \%$ and (f) $10 \%$ respectively.

Figure 7.5: SEM images of the films made with different biscarbamates.

Figure 7.6: Optical micrographs of the films heated to the melting 


\section{List of Schemes, Tables and Figures continued}

temperature of the respective biscarbamate and cooled. The images for samples

that were kept at $T_{m}$ for 2 minutes (left) and 30 minutes (right) are shown. 


\section{List of Abbreviations}

\begin{tabular}{ll} 
DLS & Dynamic Light Scattering \\
MatStudio & Material Studio \\
PDMS & Polydimethyl siloxane \\
PTCDI & Perylene-3,4.9,10-tetracarboxylic diimide \\
MJ-PTCDI & MonoJeffaminemonoisopropyl Perylene tetracarboxylicacid diimide \\
DJ-PTCDI & Jeffamine perylene tetracarboxylicacid diimide \\
LCST & Lowest Critical Solution Temperature \\
DMAc & N,N-dimethylacetamide \\
DMF & Dimethyl Formamid \\
THF & Tetrahydrofuran \\
TCE & Tetrachloroethane \\
$\alpha_{\text {agg }}$ & Mole Fraction of aggregates \\
LC & Liquid Crystal \\
PTDA & 3,4,9.10-Perylene tetracarboxylic dianhydride \\
PJ-PTCDI & MonoJeffaminemonopropyl Perylene tetracarboxylicacid diimide \\
HJ-PTCDI & MonoJeffaminemonohexyl Perylene tetracarboxylicacid diimide \\
OJ-PTCDI & MonoJeffaminemonooctyl Perylene tetracarboxylicacid diimide \\
$\AA$ & angstrom or 1x10-10 meters \\
PS & Polystyrene \\
PS-PTCDI-PS & Polystyrene perylene tetracarboxylicacid diimide \\
& $1,4,8,11,15,18,22.25-$ Octabutoxy-29H.31H-phthalocyanine \\
\hline
\end{tabular}




$\begin{array}{ll}\text { BPAPC } & \text { Bisphenol A polycarbonate } \\ \text { PMMA } & \text { Polymethylmethacrylate } \\ \text { CHL } & \text { Chloroform } \\ \text { HBC } & \text { Hexa benzene coronene } \\ \text { M }_{\mathrm{w}} & \text { Weight average molecular weight } \\ \text { OM } & \text { Optical Microscopy } \\ \text { SEM } & \text { Scanning Electron Microscopy } \\ \mu \mathrm{m} & \text { micrometer } \\ \text { rpm } & \text { rotation per minute } \\ \text { cp } & \text { centipoise } \\ \text { TPD } & \text { N,N'-diphenyl-N.N'-bis(3-methylphenyl)-[1,1 '-biphenyl]-4.4'- } \\ \text { TTA } & \text { biphenyl]-4.4'-diamine diamine } \\ & \text { tri-p-tolylamine }\end{array}$




\section{List of Appendices}

Appendix A FTIR

Appendix B NMR

Appendix C Photographs of Solutions 


\section{Chapter I Introduction}




\subsection{Self-assembly}

Self-assembly is the key process in nature by which various levels of organization are maintained to control the different functionalities very selectively. Learning from the nature, researchers all over the world have been using the same concept of utilizing secondary interaction moieties. ( $\mathrm{H}$ - bonding, $\pi-\pi$ interaction, dipole-dipole, columbic and van der Waals etc.) known as self assembling codes, to control the morphology to achieve control over the properties for a specific application. ${ }^{1}$ While a covalent bond breaking associates with a few hundred $\mathrm{kcal} / \mathrm{mole}$ of energy, the above mentioned secondary interaction only counts 1 to $5 \mathrm{kcal} /$ mole of energy. ${ }^{2}$ Two types of selfassembly codes have been used prevalently for designing synthetic systems, namely, $\pi-\pi$ and $\mathrm{H}$-bonding interactions.

Morphology of a self assembling system is the final state of stable interaction originated and directed by the self assembling codes of different molecular species. Since, self-assembly is a process of multilevel organization of the interacting entity, the direction and growth of organization are guided by the molecular design, conformation, interaction with the solvent and the presence and variation of self assembling codes. The beauty of controlling the morphology comes from the importance of controlling the properties of the self assembled systems. This also gives enhanced property of the same molecular entity. Self-assembly often associates with new functionalities. The remarkable and diversified applications with new and unexplored dimensions are being made each day by self-assembly. Another aspect of controlling the self-assembly is related to the length scale of morphology which can be varied from nano to micrometer in all the 
dimensions. So, designing the molecular entity to guide the interaction to a specific morphology is easily obtainable through self-assembly which is not possible to execute by any other physical or chemical methods. Current research on self-assembly displays an unbelievable achievements of controlled morphologies (figure 1.1) by various self assembling organic and biological molecules to fibers ${ }^{5,6}$,

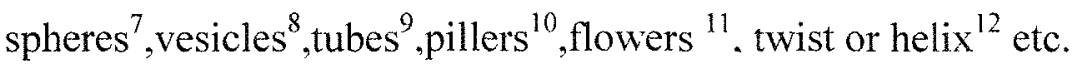
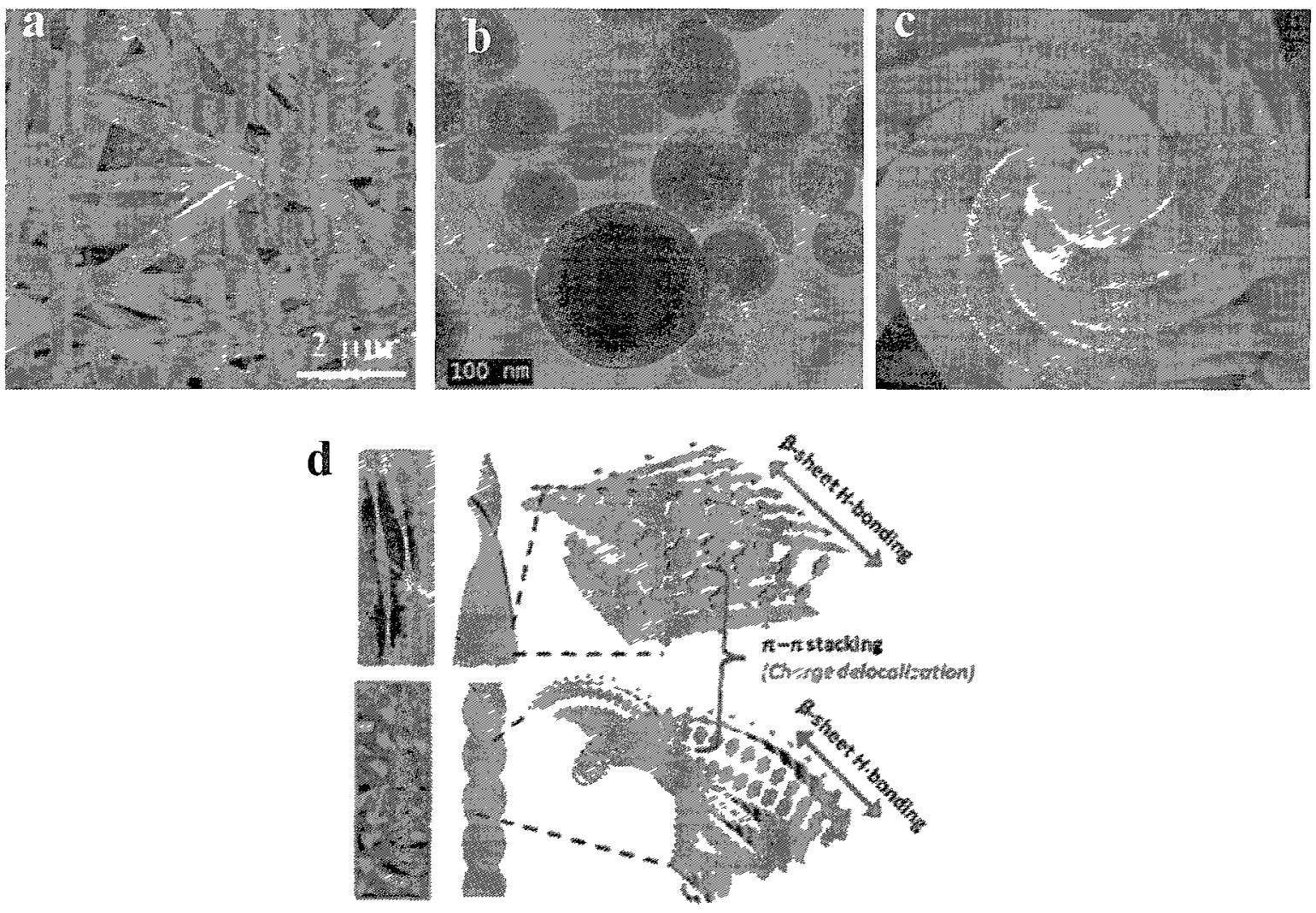

Figure 1.1: a) SEM images of Nano-fiber ${ }^{5 a}$ and b) Nano-sphere morphologies from amphiphilic perylene diimides c) SEM images showing the morphogenesis of helical patterns from epoxy nano-bristle ${ }^{10 a}$ d) Assembly of dipeptide-naphthalene diimide into lefthanded fibers (top) and ribbons (bottom) ${ }^{12 \mathrm{e}}$. 


\subsection{Current Research on Self-assembly and Morphology}

Apart from the hydrogen bonding systems, the most studied systems in the field of self-assembly are the extended planar, linear conjugated $\pi$-systems. The derivatives of perylene and phthalocyanine are the materials of most interest in this aspect due to their potential applications in electronics, biological imaging and others. ${ }^{3,4}$ They are well known for their electronic properties along with their thermal and photolytic stability. Their semiconductor and liquid crystalline properties add extra value to study the selfassembly and morphology. A planar molecule with $\pi$-conjugated system can be considered a disc-like molecule and can assemble into a long, one dimensional aggregates through their strong $\pi-\pi$ interaction. Due to the use of $\pi$-conjugated system in optoelectronic systems and the desire to fabricate molecular devices the detailed understanding of the supramolecular interaction of the individual $\pi$-system is the most active area of research in material science today. ${ }^{1}$ The self-assembly of these $\pi$-systems can be controlled either in solid state or in solution to get different morphologies by attaching side groups or chains of different types. There have been a number of studies on the self-assembly of perylene diimides with different types of small groups attached at its imide nitrogen or bay position.

Another area of intense activity is the self-assembly of amphiphilic block copolymers, with hydrophilic and hydrophobic blocks, e.g., polyethylene oxide (PEO) and polystyrene (PS) respectively, as shown in figure 1.2. When a good solvent for one of the blocks is used, the other block aggregates to form various morphologies such as 
micelles, vesicles, lamellar vesicle etc, depending on the relative lengths of the blocks, types of solvent etc. This is a well studied field in the past couple of decades. ${ }^{7 b}$

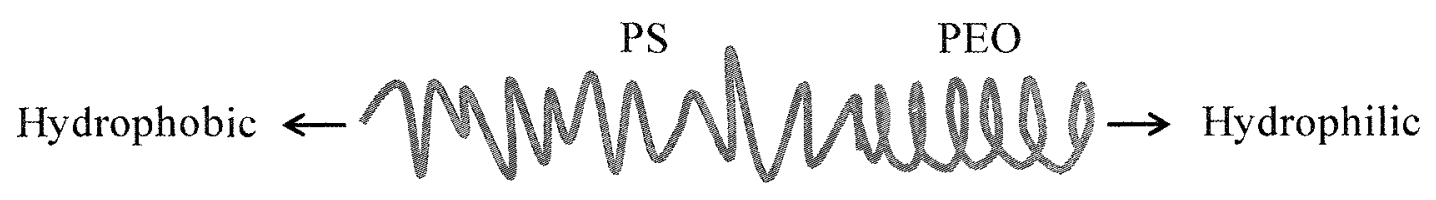

Figure 1.2: Schematic of comparison of block copolymer approach and chemical confinement approach.

Studies on morphologies in confined geometries have been developing during the past decade. Reactions that are "confined" in the molecular flasks have been reviewed." Thin films of polymers and block copolymers are considered confined, as they are restricted to the plane two dimensionally, the third (thickness) being of the same order as the coil dimensions. ${ }^{16-22}$ Other example includes crystallization in ultra thin polymer films ${ }^{16}$ and in small nanodomains ${ }^{17}$ along with the study on polymer diffusion ${ }^{18}$ and dynamics ${ }^{19}$ under confinement. conformation and molecular mobility of polymers confined in nanochannels ${ }^{11}$ (e.g., in urea), etc.

\subsection{Self-assembly in a Confined State}

In this thesis, we combined the elements of self-assembly, amphiphilic polymers and the confined state. While studies on amphiphilic block copolymers employed hydrophilic and hydrophobic segments, we replaced one of the segments with a large aromatic molecule, namely perylene diimide. Thus, the self-assembly of perylene diimide via $\pi$ interactions would occur when it is chemically confined to the polymer. We use either a water soluble polyether amine $\left(\right.$ Jeffamine $^{\mathbb{B}}$ ) or polystyrene as the polymer 
segment. The perylene diimide does not dissolve in any common solvents. With the molecular weight of polymers used here, the relative molecular weight of the aromatic segment is about one-fifth that of the polymer. This may be termed a pseudo diblock rodcoil polymer. It is also an inverse macromolecular surfactant, since the 'aromatic head group' is not water soluble, and the tail (Jeffamine ${ }^{(\mathrm{RB})}$ ) is (figure 1.3a). We also chemically confined the perylene diimide by attaching Jeffamine ${ }^{\circledR}$ to both of its sides. This is then a pseudo triblock coil-rod-coil polymer. With Jeffamine ${ }^{\text {俉 }}$ attached to both sides of the aromatic segments, this is also a Gemini Surfactant.

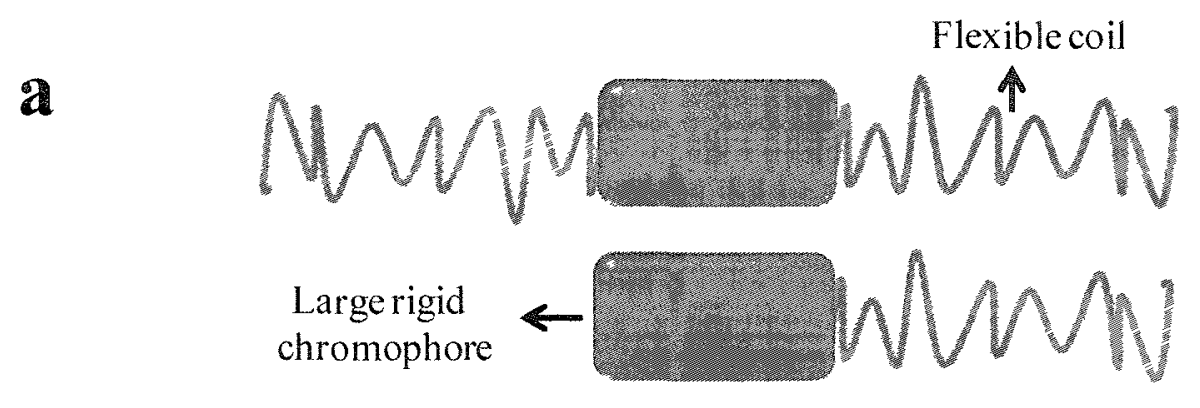

Chemically confined chromophore

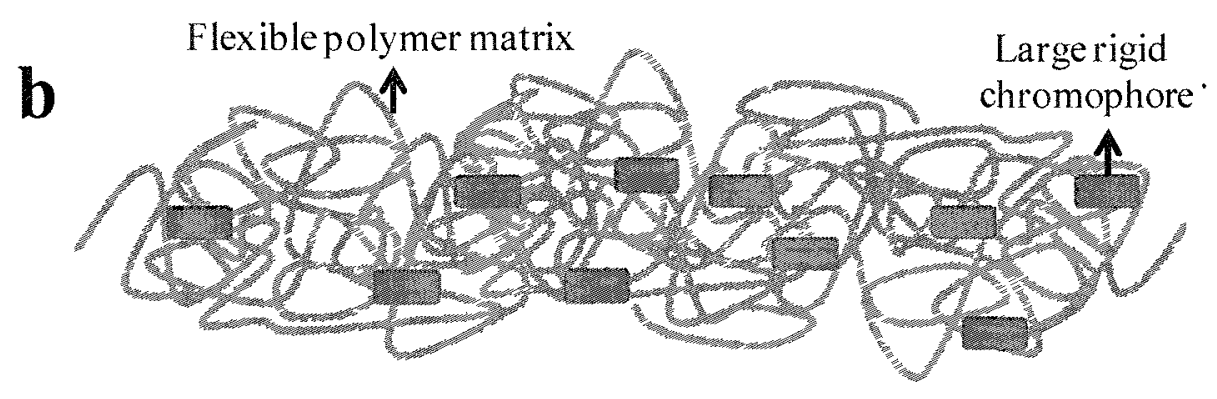

Physically confined chromophore

Figure 1.3: Schematic of polymer confined state. A chromophore is (a) confined in polymer matrix by covalent bond and (b) confined without covalent bond.

We also studied self-assembly under physical confinement. Dispersing functional small molecules is a polymer matrix (figure 1.3b) is a well known method of device 
fabrication for opto-electronic applications. ${ }^{13}$ For example, a typical photoreceptor in a copier/printer consists of photogenerating as well as hole transporting molecules dispersed in a polymer matrix. ${ }^{14}$ These are physical mixtures and normally, no specific interactions occur between the polymer and the small molecules. The hole transport molecule for example, is of triphenyl amine type, and does not possess any self assembling code mentioned above. In the present study, we dispersed self assembling molecules in polymer matrices, and studied their self-assembly and morphology. Thus, the self-assembly occurs when the molecule is physically confined in a polymer. We used a soluble phthalocyanine ( $\pi$ interaction code) and biscarbamates (hydrogen bonding code) with polycarbonate and poly (methyl methacrylate) as matrices and during phase separation occur, the small molecule diffuse to the surface and crystallize.

We found that the self-assembly of functional molecules with $\pi$ - or H-bonding moiety can be induced, influenced or controlled to get various morphologies in a polymer confined state by concentrations, solvent systems, temperature and $\mathrm{pH}$. The research work described here highlights the potential fine-tuning of phase separation of photo functional molecules in a polymer matrix. This work represents an exciting contribution of interest for a variety of researchers from materials science to organic chemistry for finding applications in drug delivery by host-guest relation, biological imaging and optoelectronic devices.

\subsection{Materials Used and Their Properties}

\subsubsection{Perylene and Perylene Diimides}

There has been a considerable interest in the development of highly conjugated organic materials for use in molecular sensors and electronics, flat panel displays, solid- 
state lighting, and light weight photovoltaic. Perylene and their derivatives, primarily perylene 3,4,9,10-tetracarboxylic diimides are one class of conjugated system that has received particular attention for these applications. These compounds absorb and emit in the visible region of the spectrum and have large photoluminescence and electroluminescence efficiencies, making them well suited for many of the applications mentioned.

The chemistry of the perylene bisimides started with the work of Kardos in 1913. ${ }^{24,25}$ After 1950, high grade perylene bisimides were found for industrial applications as pigments (especially in automotive finishes) due to the favorable combination of insolubility and superior stability, light- and weather-fastness, thermal stability and chemical inertness.

Perylene diimides combine a strong absorption in the visible region with a fluorescence quantum yield near unity and a high stability towards photooxidation. ${ }^{26-29}$ Furthermore, perylene diimides feature a relatively low reduction potential, which enables their use as an $n$-type semiconductor and as an electron acceptor in photoinduced charge transfer reactions. ${ }^{30,31}$ Because of their appealing properties, perylene monoimide and perylene diimide derivatives have been utilized in various electronic and optical applications such as field-effect transistors, $^{32}$ fluorescent solar collectors, ${ }^{26,27}$ electrophotographic devices, ${ }^{28}$ dye lasers. ${ }^{29.30}$ photovoltaic cells, ${ }^{31}$ and light-emitting diodes (LEDs). ${ }^{33}$ 
During the last three decades, considerable research is going on specially on controlling the morphology of perylene dimide in various aqueous and non-aqueous solvents. The only way to do this in solution is to balance the solubility of perylene by functionalizing in bay or imide nitrogen position with different hydrophilic and hydrophobic groups or chains (figure 1.4). Even after attaching different groups in perylene the control over morphology is still a big challenge both in solution and in solid phase.

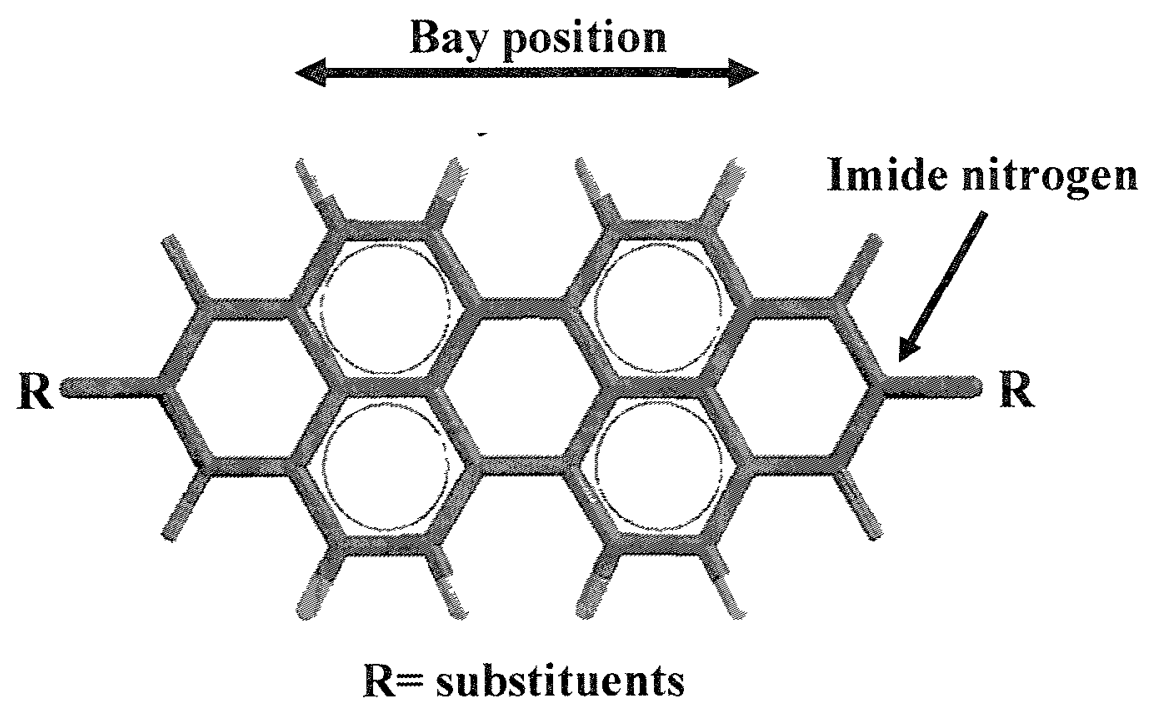

Figure 1.4: Perylene diimide skeleton highlighting bay and imide positions.

\subsubsection{Synthesis of Perylene Diimides}

The most common procedure to synthesize symmetric perylene diimides 2 is the condensation reaction of 3,4,9,10-perylenetetracarboxylic dianhydride 1 with an amine (scheme 1.1). ${ }^{35-38}$ There is a whole range of solvent for this reaction depending on the reactivity of the amine. Water, water-alcohol mixture or DMF ${ }^{37}$. DMAc ${ }^{39}$ can be used at $100-160{ }^{\circ} \mathrm{C}$ for the case of reactive amines. Solvents as quinoline or molten imidazole 
are necessary for less reactive amines such as aromatic amines with reaction temperatures of $160-180^{\circ} \mathrm{C}$.

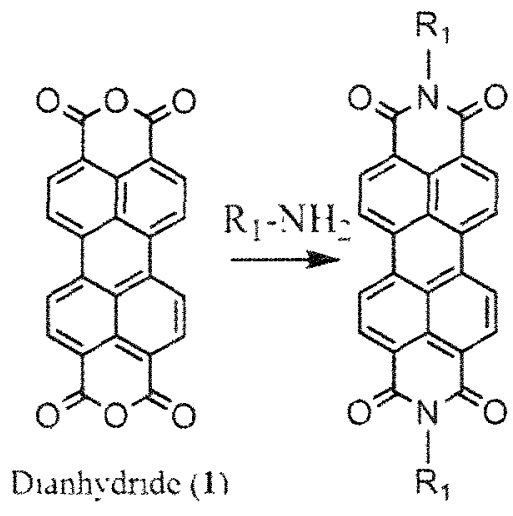

Dimide (2)

Scheme 1.1: Synthesis of symmetrical perylene diimides.

Synthesis of asymmetrically substituted perylene diimides 4 by performing the reaction with two different amines together usually does not take place because of the differences in reactivity of the two amines. Even if the reaction happens its hard to separate and purify them. Non-symmetrically substituted perylene diimides $\mathbf{3}$ are generally obtained in a multistep procedure (scheme 1.2 ). ${ }^{37,}{ }^{40}$ In this case perylene diimide can be prepared first. Diimide can be converted to mono imide mono anhydride by two different methods. Firstly, symmetrically substituted perylene diimide 2 is converted into perylene monoimide monoanhydride 3 . This can be done by reaction diimide 2 with $\mathrm{KOH}$ in tert-butanol, and subsequent treatment with acid to get monoimide monoanhydride 3 . The obtained monoimide monoanhydride 3 can be reacted with another amine to form the non-symmetrically substituted diimide 4 (scheme 1.3). Another method involves the reaction of diimide with conc. $\mathrm{H}_{2} \mathrm{SO}_{4}$ to form monoimide monoanhydride and then subsequent conversion to diimide by reacting with different amine. 


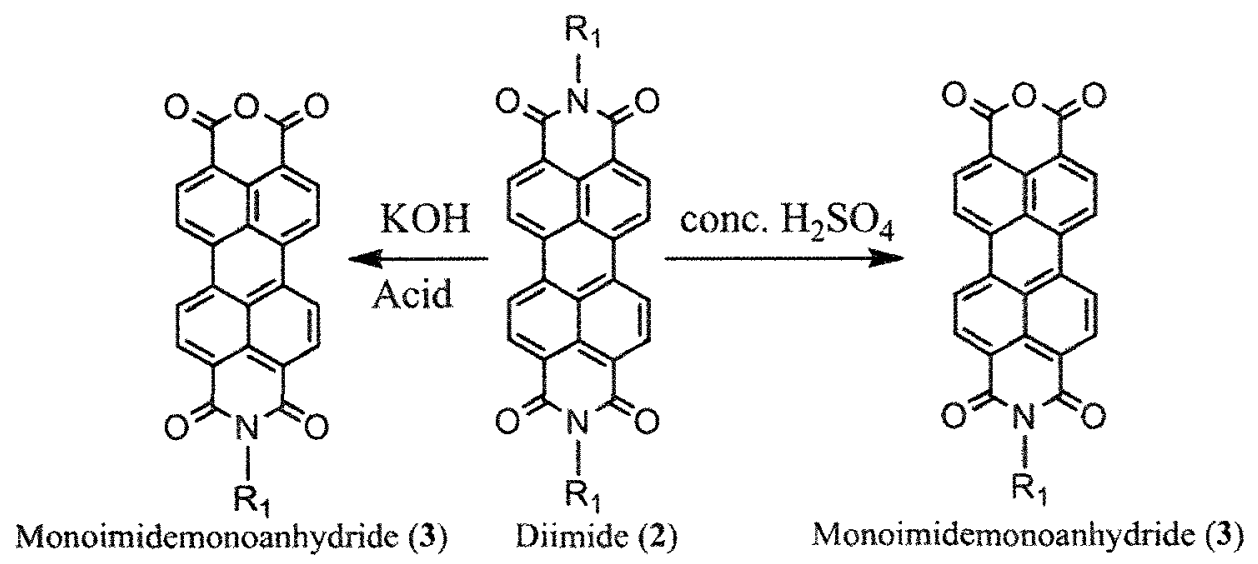

Scheme 1.2: Non-symmetrically substituted perylene diimide precursor.

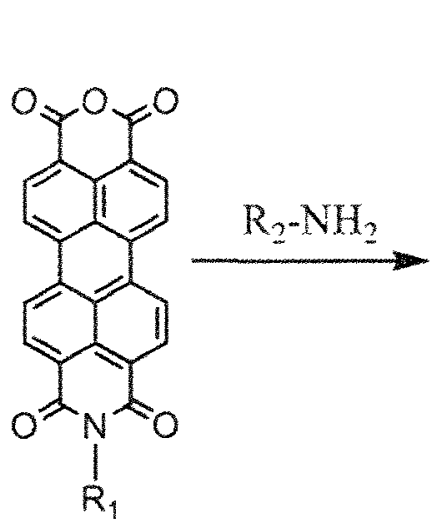<smiles>[R]N1C(=O)c2ccc3c4ccc5c6c(ccc(c7ccc(c2c37)C1=O)c64)C(=O)N([R])C5=O</smiles>

Monoimidemonoanhydride (3) Asymmetric Diimide (4)

Scheme 1.3: Synthesis of asymmetric diimide.

An alternative route to non-symmetrically substituted perylene diimides that does not require perylene bisimide as a starting material is less popular..$^{37.40}$ The monopotassium salt of dianhydride reacts in a condensation reaction with ammonia to monoimide monoanhydride. ${ }^{41}$ After reaction with another amine it gives asymmetric diimide. Another option is to react the monoanhydride monopotassium salt with an amine to get monoimide monoanhydride. This can react with another amine to give asymmetric diimide. 
Since perylene dianhydride is weakly reactive, the use of a catalyst is necessary. Typical catalysts are isoquinoline, imidazole, or even zinc salts such as zinc acetate or zinc chloride. The role of the zinc salts may be as a dehydrating agent, but is not clear. One interpretation is that the zinc salts might act as solubilizers since they may complex with the anhydride. Their solubility of dianhydride is strongly increased by the addition of zinc salts. ${ }^{37}$ The use of high boiling point solvents such as $m$-cresol. DMF, DMAc or chlorophenol etc.. is also necessary.

\subsubsection{Crystal Structure and Packing}

\subsection{Perylene Diimide Aggregation in Solid State}

The parent perylene diimide has flat $\pi$-system ${ }^{42-44}$ and can be considered as being composed of two naphthalene half units, each of which is attached to an imide unit and connected to the other naphthalene unit by two $\mathrm{Csp}^{2}-\mathrm{Csp}^{2}$ single bonds.

In the solid state, these flat platelet-like molecules then pack with each other by some longitudinal, transverse or rotational displacement. Their crystal structure shows crystallochromy i.e., color changes resulting from the different interaction of the $\pi$ systems in the crystal lattice but exhibit similar color and almost identical absorption spectra in solution. The color of a perylene dye is not only determined by the electronic properties of the individual molecule, but also is influenced by the electronic interactions with the vicinal molecules in the crystal. The crystal structure of many different perylene diimides and their derivatives has been fully studied by Graser. Hädicke and Klebe ${ }^{45}$ and Zugenmaier et $\mathrm{al}^{46}$ The distance between the $\pi-\pi$ interacting planes is 3.3 to $3.6 \AA$ (for comparison, the distance between parallel planes of graphite is $3.35 \AA$ ). ${ }^{45,47}$ 


\subsection{Perylene Diimide Aggregation in Solution}

Although the stacking of the planar $\pi$-systems of perylene diimide dyes is well documented in the crystal structures of the perylene pigments, ${ }^{42,43.45}$ only recently has the aggregation of these dyes been studied in solution. For these studies it is necessary to equip the imide nitrogen with solubilizing substituents to afford dissolution of these dyes in the polar or non polar solvents. Upon aggregation, dyes experience significant spectral changes with defined isosbestic points. The $\pi$-stacking energy is strongly reduced in more polar aromatic, chlorinated, and dipolar solvents.

In perylene pigment, it is the packing of the substituents at the imide nitrogen that controls the type and the degree of slipping of the co-facially aggregated rigid perylene unit. For one-dimensional columnar aggregates in solution these packing restrictions are absent and, therefore, the aggregation of the perylene dye is only governed by the chromophore-chromophore interactions themselves and spectral response changes with aggregation. Normally $\pi-\pi$ stacking interactions between perylene diimide dyes become stronger in alcohol and water which are equally little polarizable solvents and, therefore, cannot solvate the $\pi$-surfaces well.

\subsection{Optical, Electrochemical and LC properties}

Since the discovery of the intense yellow-green photoluminescence of the parent perylene diimides, numerous perylene diimide dyes have been synthesized for their application as fluorescent standards, in fluorescent light collectors, or as laser dyes. ${ }^{35}$ Because of the nodes of the HOMO (Highest Occupied Molecular Orbital) and 
LUMO (Lowest Unoccupied Molecular Orbital) orbital at the imide nitrogen substitution at this position has a negligible influence on the absorption and emission properties of perylene diimides. Therefore, perylene diimides can be regarded as a closed chromophoric system whose intensity and position remain unaltered by the respective imide substituents. ${ }^{48}$ Accordingly, imide substituents are well suited to tailor applicationdirected properties like solubility and to prevent aggregation that has a pronounced influence on the optical spectra.

Perylene imides are characterized by a vibronically structured strong absorption in the visible region of the spectrum and exhibit a similarly strong fluorescence. The fluorescence quantum yield is near one. Perylene diimides are fairly electron deficient dyes and classified as n-type semiconductors ${ }^{30,31}$ which are easy to reduce and rather difficult to oxidize. For most compounds, two reversible reduction waves and one reversible oxidation wave are found in cyclic voltammetry. Substituents in the bay-area have a pronounced effect on the respective redox potentials. Liquid crystallinity is another important property of perylene diimide. Only a few liquid crystalline perylene derivatives have been synthesized so far..$^{39,49}$ The electron-deficient character of perylene bisimide dyes is also a prerequisite for the high photochemical stability of these

\subsection{2 $1,4,8,11,15,18,22,25$-Octabutoxy-29H,31H-phthalocyanine}

Phthalocyanine molecules act as p-type semiconductor with a typical energy gap of less than $2.0 \mathrm{eV}$. Their semi-conducting and structural properties resemble those of 
doped polyacetylenes, and the characteristic forms of phthalocyanine molecules are monomer or aggregated units, ring stacked or polymeric sheet type. Phthalocyanines have a high light-collecting property in the visible spectrum and have the potential ability to accept or release electrons because of the extended delocalized system in its structure. They show polymorphism by taking different forms in their crystal structure. ${ }^{50}$ Taking into account the fact that phthalocyanines possess intense absorption in the visible and near-IR regions (600-900 $\mathrm{nm}$ ) and exhibit excellent chemical, light and thermal stability compared to other porphyrins, it is a very promising material in the field of opto electronic applications. ${ }^{51}$ Phthalocyanines are structurally related to other macrocyclic pigments, especially the porphyrins. Phthalocyanine skeleton has four pyrrole-like subunits linked to form a 16 -membered ring. The pyrrole-like rings within phthalocyanine are closely related to isoindole. Phthalocyanines are well known for their non solubility in almost all solvents and thus needs to be substituted either in its peripheral or non peripheral positions. ${ }^{52-60}$ Phthalocyanine can be present as substituted or unsubstituted and can be metalized or metal free.

The existing and potential applications of organic functional molecules in electronic ${ }^{51 \mathrm{e}}$ and light emitting devices ${ }^{5 / f}$ require an understanding of the self-assembly of organic small molecules in polymer matrices. The phase separation and association characteristics of these self assembling molecules from polymer matrices are different from the behavior of other molecules which do not possess the self-assembling code. In our present study we used 1,4,8,11,15,18,22.25-Octabutoxy-29H.31H-phthalocyanine which is a non planar molecule and shows a saddle conformation (figure 1.5). 

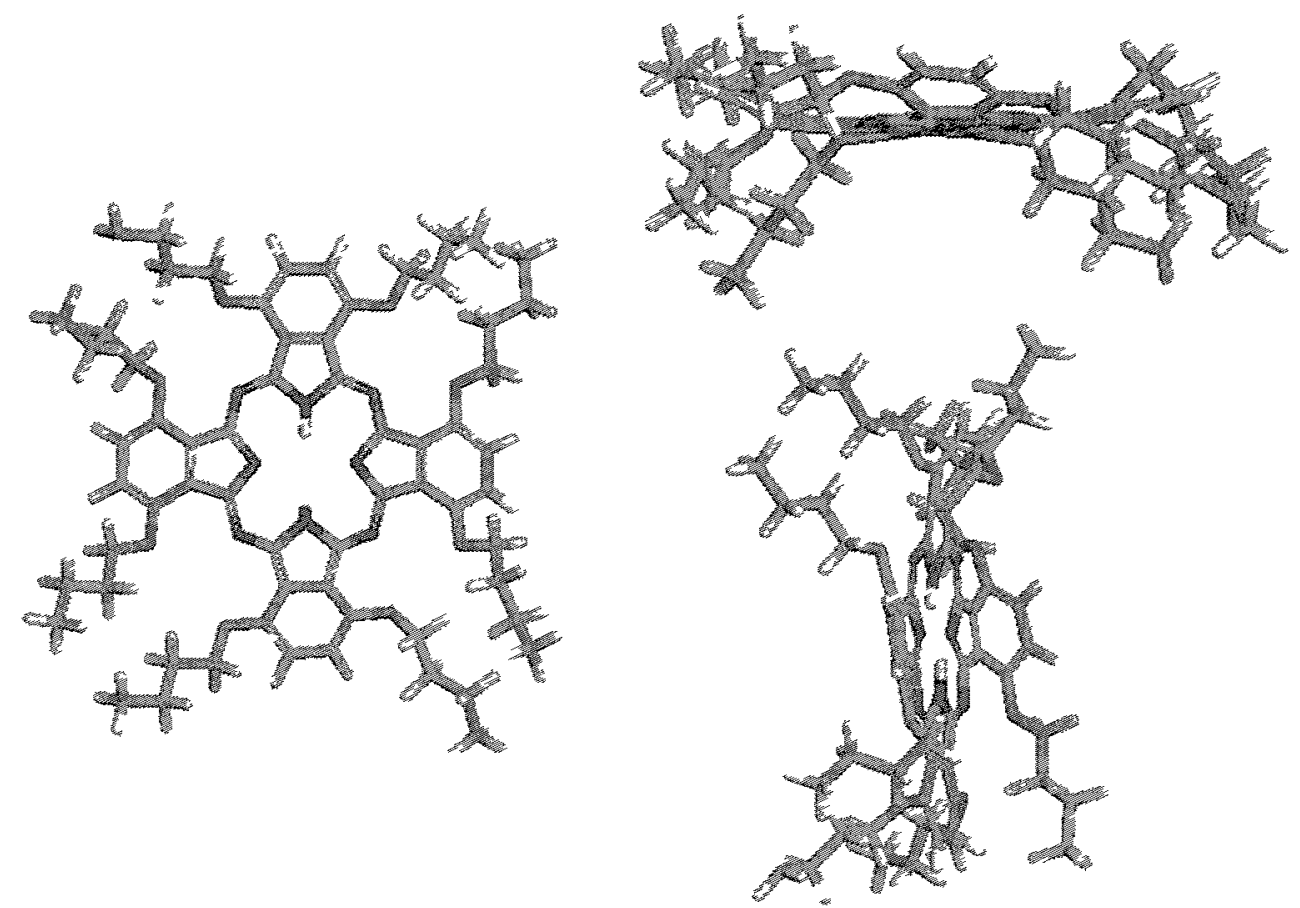

Figure1.5: Molecular Model of 1.4,8,11,15,18,22.25-Octabutoxy-29H,31H-phthalocyanine drawn by Accelrys MatStudio 3.0. Oxygen and nitrogen atoms are marked with red and blue respectively.

\subsubsection{Biscarbamates}

In recent years, there has been a growing interest to study the gelation of low molecular weight organic gelators (LMOGs, molecules whose molecular mass is $\leq 3000$ ) and their organogels in terms of morphology, properties and potential applications in membrane and separation technology, catalysis, drug delivery, fuel engineering etc. ${ }^{61}$

Carbamates and biscarbamates, as a model compound of polyurethanes, are an important class of self assembling compounds which forms gels. They can be used for increasing the abrasion resistance of polyurethane compositions, ${ }^{62}$ improving the hardness of urethane-based adhesives and sealants, ${ }^{63}$ and adjusting viscosity values of oils 
and greases when used as additives. ${ }^{64,65}$ Some carbamates and thiocarbamates have biological activity and can be utilized as insecticides. ${ }^{66}$ The association process in carbamates is similar to biological self-assembly involving hydrogen bonding. Their melt viscosity is usually of the order of a few centipoise, and they crystallize rapidly. Hence, carbamates with alkyl side chains have potential applications as vehicles in reprography and ink jet printing technologies. ${ }^{67-69}$ It has been reported that the gelation is dependent on the length of alkyl chain, spacer, method of preparation (slow cooled and quenched), and concentration etc. ${ }^{70}$

Carbamates contain single $\mathrm{H}$-bonding moiety whereas biscabamates contain two hydrogen bonding sites separated by a $\left(\mathrm{CH}_{2}\right)_{6}$ spacer and symmetrical substitution of the alkyl chains on either side (figure 1.6).<smiles>[R]OC(=O)NC(C)(C)CC(C)(C)NC(=O)O[R]</smiles>

Figure 1.6: Chemical structure of Biscarbamate. Here $R=C_{4}$ to $C_{18}$

In our present case we used biscarbamates of different length to study their selfassembly in a polycarbonate matrix.

\subsection{Concepts and Terms used in Self-assembly}

\subsubsection{Electronic Energy Level, Absorption and Emission}

Outer orbital electrons of a molecule possess higher energy than inner shell electrons and the energies are quantized. During the process of electronic transition a photon can be absorbed when its energy corresponds to the excitation of an electron to a 
more energetic state and a photon of an appropriate wavelength can be emitted as well when an excited electron drops back to a lower state. The fraction of light that absorbs during electronic transition is called absorbance and is proportional to concentration and path length. The transitions (figure 1.7) from lowest energy ground state $\left(S_{0}\right)$ to excited states $\left(\mathrm{S}_{0}, \mathrm{~S}_{1}, \mathrm{~S}_{2} \ldots \ldots .\right.$. etc $)$ are associated with photon wavelength of IR, Visible and UV band. If the electronic transition occurs in the visible range of wavelength we see color. Electronic energy levels are composed of vibrational and rotational energy levels. So, an electronic transition is always accompanied with vibrational and rotational level transition and gives a broad absorption band resulting from the superposition of vibrational and rotational transition. The emission spectrum originates from emission of photon by excited electron to get back to ground state and often a mirror image of absorption spectra (figure 1.8).

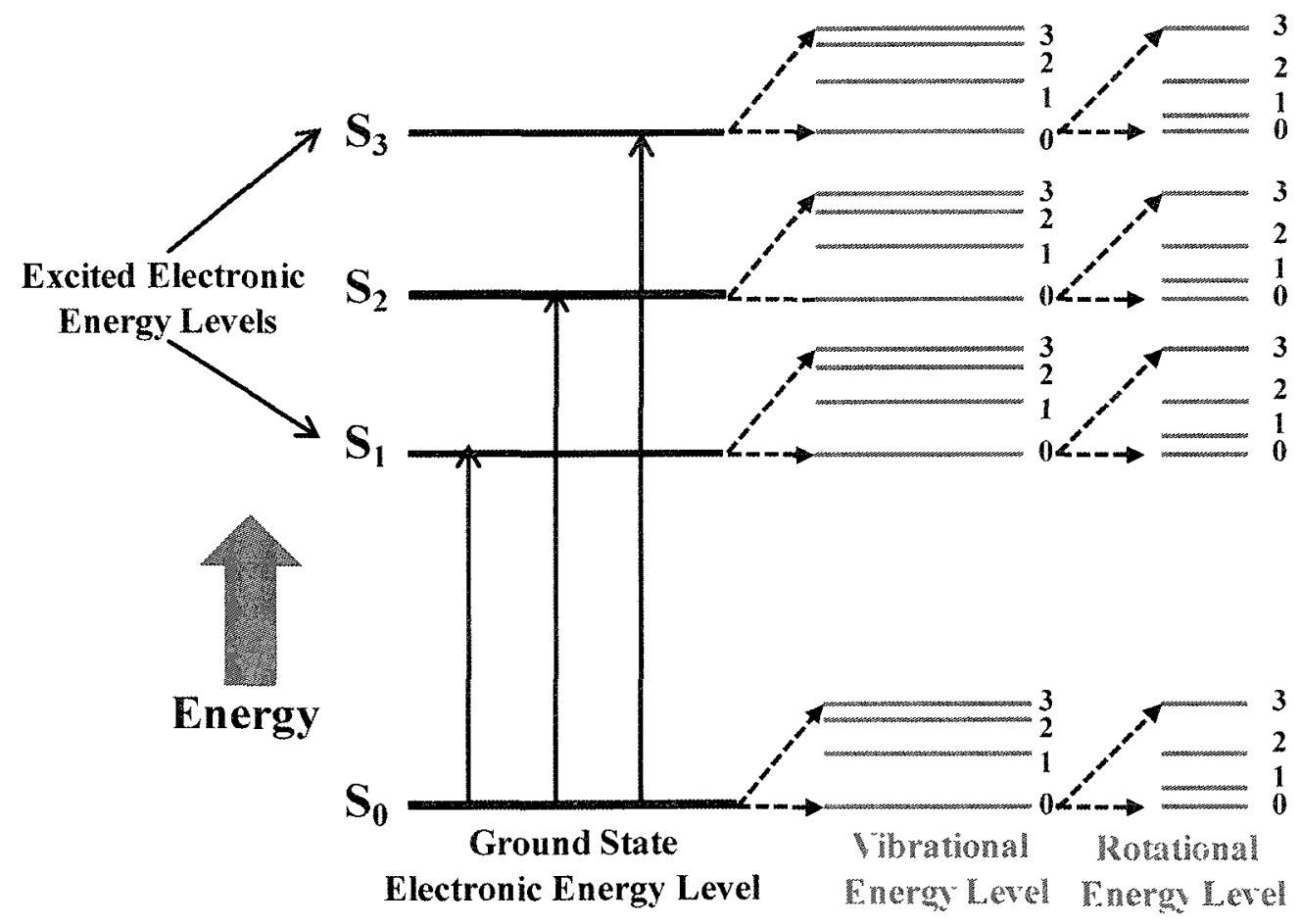

Figure 1.7: Schematic of Electronic energy levels. 

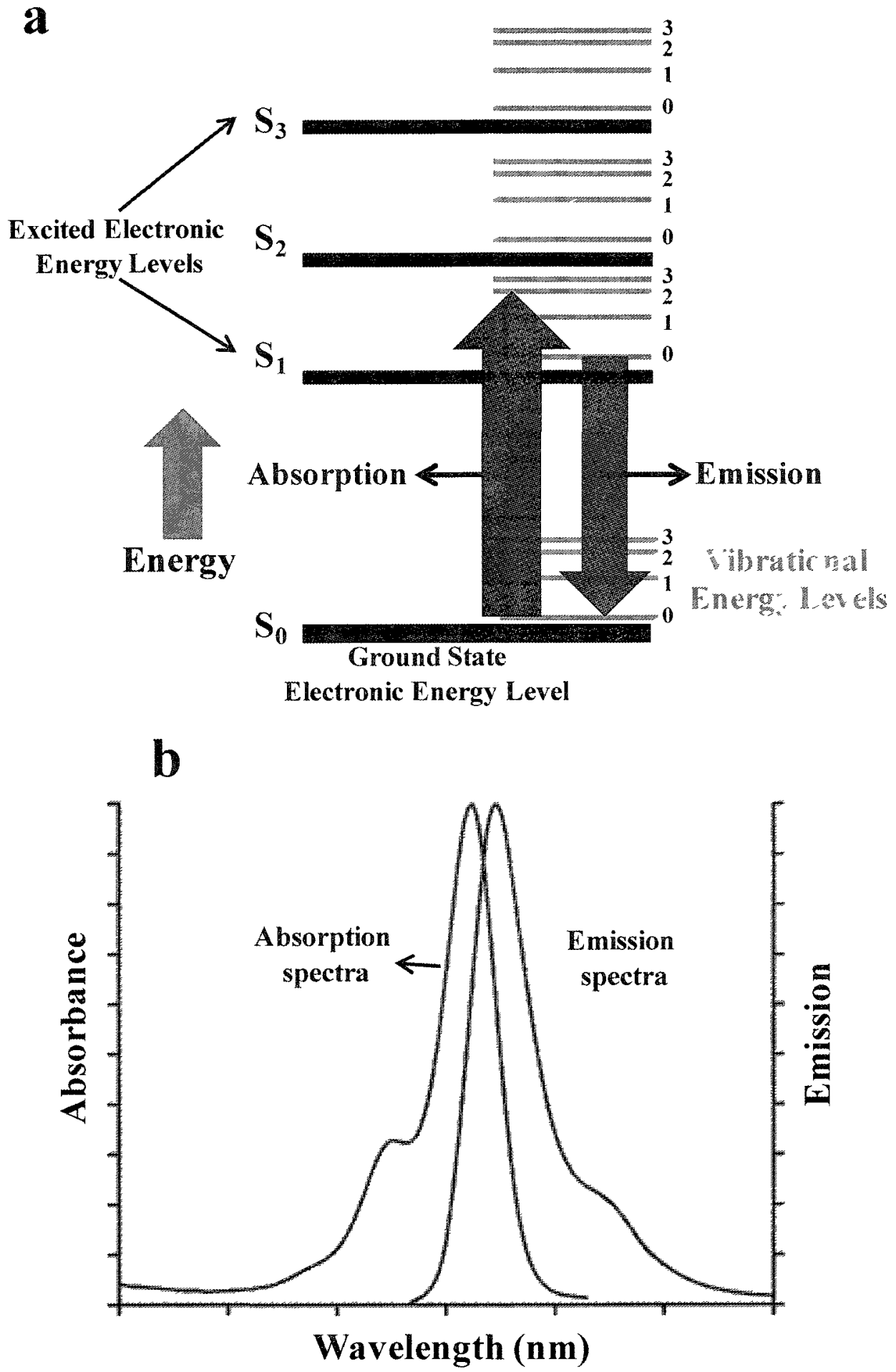

Figure 1.8: Schematic of (a) absorption and emission process and (b) corresponding spectra. 


\subsubsection{Definition of $\mathrm{H}$ and $\mathrm{J}$ Aggregates: Mode of Aggregation}

The self-association of dyes in solution or at the solid-liquid interface is a frequently encountered phenomenon in dye chemistry owing to strong intermolecular van der Waals-like attractive forces between the molecules. The aggregates in solution exhibit distinct changes in the absorption band as compared to the monomeric species. From the spectral shifts, various aggregation patterns of the dyes in different media have been proposed. The bathochromically shifted $\mathbf{J}$-bands ( $\mathrm{J}$ for Jelly, one of the first workers who investigated these shifts) and hypsochromically shifted $\mathbf{H}$-bands ( $\mathrm{H}$ for hypsochromic) of the aggregates have been explained in terms of molecular exciton coupling theory, i.e., coupling of transition moments of the constituent dye molecules. The aggregates that exhibit $\mathrm{J}$-bands in their absorption spectrum are called $\mathrm{J}$ aggregates while $\mathrm{H}$ aggregates exhibit H-bands. ${ }^{71}$

\subsubsection{Pattern of Aggregates in the Solution}

Extensive studies on J-and H-aggregates have resulted in the proposal that these aggregates exist as a one-dimensional assembly in solution that could be in (a) brickwork, (b) ladder, or (c) staircase type of arrangement. The pattern of the assembly is shown in the figure 1.9.

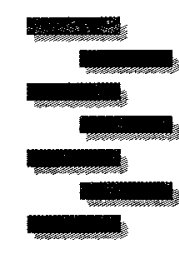

Ladder

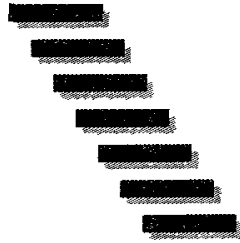

Staircase

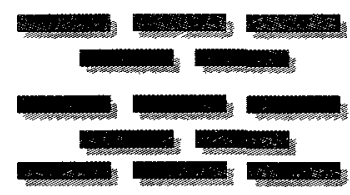

Brickwork

Figure 1.9: One dimensional assembly in solution by planar molecule. 


\subsubsection{Absorption Spectra of Aggregates}

Bathochromic shift is a change of spectral band position in the absorption, reflectance, transmittance, or emission spectrum of a molecule to a longer wavelength (lower frequency). This can occur because of a change in environmental conditions: for example, a change in solvent polarity will result in solvatochromism. Solvatochromism is the ability of a chemical substance to change color due to a change in solvent polarity. Negative solvatochromism corresponds to hypsochromic shift, positive solvatochromism corresponds to bathochromic shift with increasing solvent polarity. The sign of the solvatochromism depends on the difference in dipole moment of the molecule of the dye between its ground state and excited state. A series of structurally related molecules in a substitution series can also show a bathochromic shift. Bathochromic shift is a phenomenon seen in molecular spectra, not atomic spectra - it is thus more common to speak of the movement of the peaks in the spectrum rather than lines.

Bathochromic shifts are often referred to as red shifts. Although this usage is considered informal, it is often used in the literature. It has no relation to Doppler shift or other wavelength-independent meanings of red shift. Hypsochromic shifts are often referred to as blue shifts in photochemistry. Blue shift occurs when the wavelength with maximum intensity $\left(\lambda_{\max }\right)$ moves to the shorter wavelength and red shift occurs when $\lambda_{\max }$ moves to the longer wavelength. 
There are some other distinguishing features between $\mathrm{J}$ and $\mathrm{H}$ type stacking: in $\mathrm{H}(\mathrm{J})$-aggregates the ratio of the first two vibronic peak intensities in the absorption spectrum decreases (increases) with increasing excitonic coupling, while the ratio of the $S_{0 \text { - }}$ ${ }_{0}$ to $\mathrm{S}_{0-1}$ emission intensities increases (decreases) with disorder and increases (decreases) with increasing temperature. ${ }^{72}$

\subsubsection{Theory}

\subsubsection{The Exciton Theory}

According to exciton theory, the dye molecule is regarded as a point dipole and the excitonic state of the dye aggregate splits into two levels through the interaction of transition dipoles. The dye molecules may aggregate in a parallel way (plane-to-plane stacking) to form a sandwich-type arrangement (H-aggregate) or in a head-to-tail arrangement (end-to-end stacking) to form a J-aggregate. A transition to the upper state in parallel aggregates having parallel transition moments and to a lower state in a head-to-tail arrangement with perpendicular transition moments leads to hypsochromic (red) and bathochromic (blue) shifts, respectively (figure 1.10). 


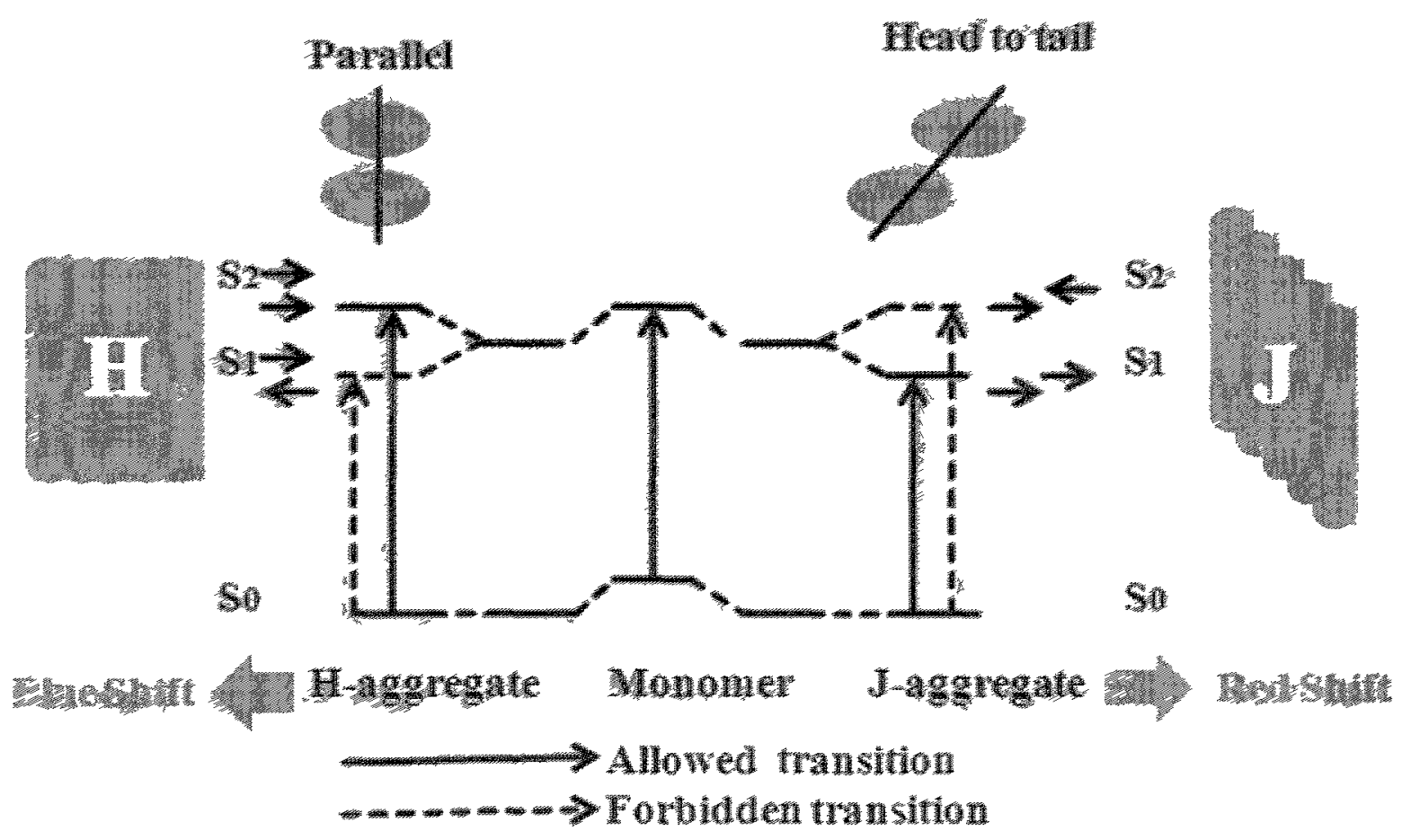

Figure 1.10: $\mathbf{H}-$ and $\mathbf{J}-$ type stacking, geometry and absorption pattern.

The angle between the line of centers of a column of dye molecules and the long axis of any one of the parallel molecules is called the 'angle of slippage'(R), Large molecular slippage $\left(\mathrm{R}<54.7^{\circ}\right)$ results in a bathochromic shift ( $\mathrm{J}$-aggregate). and small slippage $\left(\mathrm{R}>54.7^{\circ}\right)$ results in a hypsochromic shift (H-aggregate). Important point to note is that, the exciton theory is valid only when the interaction between orbitals of constituent molecules is negligible.

\subsubsection{The Nature and Origin of $\pi-\pi$ Interaction}

Attractive interactions between $\pi$-systems have been known for the last few decades. This interaction controls diverse phenomena such as the vertical base-base 
interactions which stabilize the double helical structure of DNA, the intercalation of drugs into DNA, the packing of aromatic molecules in crystal, the tertiary structures of proteins, the conformational preferences and binding properties of polyaromatic macro cycle, complexation in many host-guest systems and porphyrin aggregations.

Strong attractive interactions between two porphyrins lead to aggregation in solution. Even if we say it as $\pi-\pi$ interaction, its not as simple as it suggests. There are some previous models ${ }^{73}$ which describe the origin and nature of $\pi-\pi$ interaction but those models are not either complete or case specific namely, solvophobic model (attraction is considered as entropic), electron donor-acceptor model and atomic charge model etc. (attraction from uneven charge distribution across the $\pi$-system). The most successful and comprehensive understanding comes from the electrostatic model ${ }^{74}$ which covers almost all aspect of $\pi-\pi$ interaction.

Electrostatic model describes that the major contributions to the interaction energy come from the electrostatic and van der Waals components where its considers induction as a second-order term. ${ }^{74}$ The apparent energy of interaction between two molecules in solution includes association of the two molecules and displacement of solvent. In non-polar organic solvents, the electrostatic interactions with the solvent will be negligible, and so the dominant electrostatic interaction will come from the association energy. However, both the association and desolvation energies are likely to be associated with significant van der Waals interactions. This model indicates that the geometries of $\pi-\pi$ 
interactions are controlled by electrostatic interactions but that the major energetic contribution comes from other factors. It is a $\pi-\sigma$ attraction rather than a $\pi-\pi$ electronic interaction which leads to favorable interactions. These electrostatic effects determine the geometry of interaction, while van der Waals interactions (and solvophobic effects) make the major contribution to the magnitude of the observed interaction.

\subsection{Thesis Objectives}

The overall objective of this thesis was to investigate the self-assembly and morphology of small functional organic molecule with self assembling codes such as $\pi$ - or $\mathrm{H}$ - bonding moiety in a polymer matrix where a chromophore was confined chemically by attaching hydrophilic and hydrophobic polymer or short alkyl groups to study the selfassembly in solution. The confinement was done physically without covalent bond by spreading self assembling molecule in a polymer matrix to study their self-assembly in solid state films as well. We know that the properties of the molecule can be controlled if we can control the morphology and the morphology can be controlled through self assembly. This study of self-assembly in a confined state by small functional molecules with $\pi$ - or H-bonding moiety would find applications in fabricating photo-generating devices for optoelectronic and solar cell applications. fluorescence probes in biological applications, drug delivery by incorporating guest molecule in hydrophobic $\pi$-system by supramolecular interaction. So, based on the main objectives of the research our initial aim was set to study the self assembly and morphologies of a highly fluorescent photoconducting blocks with $\pi$-bonding moiety in a polymer matrix. We explored the selfassembly in confined state by the following sequences: 
1. Synthesis and morphology of water soluble pseudo-block copolymer of rod-coil type of perylene diimide by attaching jeffamine and alkyl groups.

2. The effect of alkyl chain length of amphiphilic block copolymers of perylene diimide on the morphology.

3. Synthesis and morphology of non aqueous self-assembly of polystyrene jacketed symmetric perylene diimide.

4. Self-assembly in a physically confined state by substituted metal free phthalocyanine with $\pi$-bonding moiety in polymer matrix.

5. H- bonding mediated self-assembly of symmetric biscarbamate in a polycarbonate matrix.

\subsection{Thesis Outline}

This thesis is divided into eight chapters. In chapter I, we have discussed about the self-assembly, its importance and current trend of research on self-assembly and how the idea of self assembly in a polymer confined state can be considered as a pseudo block copolymer system. We have also discussed about the synthesis of perylene diimide and general information and properties of the materials used in the thesis.

Chapter II describes the common instrumental techniques used to characterize and analyze self-assembly. 
In Chapter III, the morphology and packing of perylene diimides (PTCDI), with alkyl group in one side and Jeffamine ${ }^{\mathbb{B}}$ in the other side (MJ-PTCDI) and Jeffamine ${ }^{\mathbb{B}}$ on both sides of perylene (DJ-PTCDI) are described. Here the alkyl group is isobutyl and Jeffamine $^{(\mathbb{R}}$ is block copolymer of polyethylene oxide and polypropylene oxide $\left(M_{\mathrm{W}}=\right.$ 2000). We report here that the aggregation of both of the diimides is dependent on concentration and solvent. The aggregation in aqueous media starts at $10^{-5} \mathrm{M}$ for M.JPTCDI and at $10^{-7} \mathrm{M}$ for DJ-PTCDI. These diimides don't give any aggregation in non aqueous solvent but evaporation induced self-assembly is reported. Their absorption spectra shows that the packing mode of DJ-PTCDI is J-type whereas MJ-PTCDI aggregates as H-type. X-ray diffraction patterns show changes in the number of reflections. Their aggregate structure shows high thermal and pH stability.

Chapter IV describes the effect of side chain length of hydrophobic alkyl group on the self-assembly of amphiphilic perylene diimide. The target was to change the hydrophilic-hydrophobic ratio to control the self-assembly of perylene diimides in water. They are all water soluble and their stacking is concentration dependent. For shorter alkyl group folded sheet morphology is seen which changes to tube for longer chain. Their crystal structure is different giving different X-ray profile. Annealing doesn't change the packing profile for PJ-PTCDI and OJ-PTCDI but changes for HJ-PTCDI which may be due to crystal-LC transition. The DSC doesn't give any idea of LC transition because of long chain polymer. Non aqueous solvent induces different morphology during evaporation for all the diimides. 
In chapter $\mathrm{V}$, the self-assembly and morphology of perylene diimide jacketed by polystyrene was studied in non aqueous solvents in line with our previous work on perylene diimide based on PDMS either in one side or both sides. PS-PTCDI-PS does not show any sign of aggregation in non aqueous solvents but self assembles in drop cast films showing spherical or web like morphologies. It also indicates the formation of aggregate structures in mixed solvent systems.

Chapter VI describes the self-assembly of octabutoxy substituted phthalocyanine (Pc) which is controlled by solvent, polymer matrix and process of film making. Here we used phthalocyanine with $\pi$-bonding motif as a functional organic molecule to disperse in polymer matrix. We prepared films with PMMA, BPAPC (Bisphenol A polycarbonate) and PS by dissolving Pc in into two different solvents. TCE and Chloroform. We report here the formation of needle in PMMA and BPAPC with TCE. Whereas films made with chloroform by PMMA or BPAPC show sub-surface droplet like morphology.

In chapter VII, we explored the self-assembly of another set of molecules with different side chain length. Here we used biscarbamate, a synthetic analogue of polyurethane as a self assembling entity. These molecules have two H-bonding moieties separated by a spacer of constant length. The films were made by dissolving them in chloroform and BPAPC. We report that with shorter side chain length they form donut shape morphology which turns to needle with higher concentration whereas with intermediate chain length a protrusion of crystal is formed with domain and with very long 
chain they form needle like crystals. The crystals formed in polymer matrix are thermally nonreversible.

Chapter VIII represents the outcome of the research on self-assembly in a confined state with some potential applications and recommendations of some unexplored future directions. 


\subsection{References:}

1. (a) Rep, D. B. A.; R. Roelfsema, van Esch. J. H.; Schoonbeek, F. S.; Kellogg, R. M.;

Feringa, B. L.; Palstra, T. T. M.; Klapwijk, T. M. Adv. Mater. 2000, 12, 563. (b)

Brunsveld, L.; Zhang, H.; Glasbeek, M.; Vekemans, J. A. J. M.; Meijer. E. W. J. Am.

Chem. Soc. 2000, 122, 6175. (c) Schenning. A. P. H. J.; Jonkheijm, P.; Peeters, E.;

Meijer, E. W. J. Am. Chem. Soc. 2001, 123. 409. (d) Peeters. E.; van Hal, P. A.; Meskers,

S. C. J.; Janssen, R. A. J.; Meijer. E.W. Chem. Eur. J. 2002, 8, 4470. (e) Wurthner, F.;

Thalacker, C.; Sautter, A.; Schartl, W.; Ibach, W.; Hollricher, O. Chem. Eur. J. 2000, 6, 3871.

2. (a) Hoeben, F. J. M.; Jonkheijm, P.; Meijer, E. W.; Schenning, A. P. H. J. Chem. Rev. 2005, 105, 1491. (b) Lehn, J.-M. Science 2002, 295, 2400. (c) Steed, J. W.; Atwood, J. L. Supramolecular Chemistry: Wiley \& Sons: Chichester, 2000. (d) Sinnokrot, M. O.; Cherrill, C. D. J. Am. Chem. Soc. 2004, 126, 7690.

3. (a) Bourroughes. J. H.; Bradley, D. D. C.: Brown, A. R.; MacKay, R. N.; Friend, R. H.;

Burn, P. L.; Homes, A. B. Nature 1990, 347, 539. (b) Sariciftci, S.; Smilowitz, L.;

Heeger, A. J.; Wudl, F. Science 1992, 258, 1474. (c) Halls, J. J. M.; Walsh, C. A.; Greenham, N. C.; Marseglia, E. A.; Friend, R. H. Nature 1995, 376, 498. (d) Sirringhaus, H.; Brown, P. J.; Friend, R. H.; Nielsen, M. M.; Bechgaard, K.; Langeveld-Voss, B. M. 
W.; Spiering, A. J. H.; Janssen. R. A.; Meijer, E. W.; Herwig. P.; de Leeuw, D. M. Nature 1999, 401, 685.

4. (a) van de Craats, A. M.; Warman, J. M. Adv. Mater 2001. 13, 130. (b) van de Craats. A. M.; Warman. J. M.; de Haas, M. P.; Adam, D.; Simerer, J.; Haarer, D.; Schuhmacher, P. Adv. Mater. 1996, 8, 823. (c) van Nostrum, C. F.: Nolte, R. J. M. Chem. Commun. 1996. 21, 2385. (d) Smolenyak. P.; Peterson. R.; Nebesny, K.; Torker. M.: OBrien, D. F.; Armstrong. N. R. J. Am. Chem. Soc. 1999, 121, 8628. (e) van de Craats. A. M.; Warman, J. M.; Mullen, K.; Geerts, Y.; Brand, J. D. Adv. Mater. 1998, 10, 36. (f) Liu, C.-Y.; Fechtenkotter, A.; Watson, M. D.: Mullen. K.; Bard, A. J. Chem. Mater. 2003, 15, 124. (g) Liu, C. Y.: Pan, H.-L.; Fox, M. A.; Bard. A. J. Science, 1993, 261. 897. (h) SchmidtMende, L.; Fechtenkotter, A.; Mullen. K.; Moons. E.; Friend, R. H.; MacKenzie, J. D. Science 2001. 293. 1119.

5. (a) Balakrishnan, K.; Datar. A.; Naddo, T.; Huang, J.; Oitker, R.; Yen, M.; Zhao, J.; Zang, L. J. Am. Chem. Soc. 2006. 128, 7390. (b) Briseno, A. L.: Mannsfeld, S. C. B.; Reese, C.; Hancock. J. M.; Xiong. Y.; Jenekhe. S. A.: Bao, Z.: Xia, Y. Nano Lett. 2007, 7, 668. (c) Ryu, J.-H.; Hong, D.-J.; Lee, M. Chem. Commun. 2008, 9,1043.

6. (a) Balakrishnan, K.; Datar, A.; Oitker. R.; Chen, H.: Zuo, J.; Zang, L. J. Am. Chem. Soc. 2005, 127, 10496. (b) Kang. S. H.; Pokroy, B.; Mahadevan, L.; Aizenberg, J. ACS Nano, 2010, 4, 6323. (c) Carneiro, K.; Sleiman, H. F. J. Am. Chem. Soc. 2009. 132, 679. 
7. (a) Lu, J.; Wu, L.; Jiang, J.; Zhang, X. Eur. J. Inorg. (hem. 2010, 4000. (b) Discher, D. E.; Eisenberg, A. Science 2002, 297.

8. (a) Yao, D.: Bender, T. P.; Gerroir, P. J.; Sundararajan. P. R. Macromolecules 2005. 38, 6972. (b) Metera, K.; Sleiman. H. F. Macromolecules, 2007, 40, 3733. (c) Zhang, X.; Rehm, S.; Safont-Sempere, M. M.: Wurthner, F. Nature Chemistry, 2009. 1.623 .

9. (a) Wilner. O. I.; Henning. A.; Shlyahovsky, B.; Willner, I. Nano Lett., 2010, 10, 1458.

(b) Alday F. A.; Lo. P. K.; Karam, P.: McLaughlin. C. K.: Cosa, G.; Sleiman, H. F. Nature Nanotechnology, 2009, t, 349 .

10. Pokroy, B.; Kang, S. H.; Mahadevan. L.; Aizenberg, J. Science, 2009, 323, 237.

11. Karan, S.; Mallik, B. J. Phys. Chem. C 2008. 112, 2436.

12. (a) Yang, L. Y.; Shi, M. M.; Wang, M.; Chen. H. Z. Chinese Chemical Letters, 2008.

19, 1260. (b) Kimura. M.; Hatanaka,T.; Nomoto, H.: Takizawa, J.; Fukawa. T.; Tatewaki, Y.; Shirai, H. Chem. Mat. 2010, 22, 5732. (c) Li, C.; Deng, K.; Tang, Z.; Jiang, L. J. Am.

Chem. Soc. 2010, 132, 8202. (d) Rao, K. V.; George, S. J. Organic Letters. 2010, 12, 2656. (e) Shao, H.; Nguyen. T.; Romano, N.C.; Modarelli, D.A.; Parquette, J. R. J. Am. Chem. Soc., 2009. 131.16374.

13. Dudowick, J.; Douglas, J.F.; Freed. K. F.; J. Phys. Chem. B. 2009, 113, 3920.

14. (a) Khan, F.; Khanna, S.; Hor , A-M.; Sundararajan, P. R. Can. J. Chem. 2010, 88 , 247. (b) Khan, F.; Hor, A.-M.; Sundararajan. P. R. Synthetic Met. 2005.150,199. (c) 
Khan, F.; Hor, A.-M.; Sundararajan, P. R. Pure Appl. Chem. 2004, 76, 1467. (d) Khan,

F.; Hor, A.-M.: Sundararajan, P. R. J. Phys. Chem. B. 2004, 108, 117.

15. (a) Murase, T.; Horiuchi, S.; Fujita, M. J. Am. Chem. Soc. 2010, 132, 2866. (b)

Yoshizawa, M.; Klosterman, J. K.; Fujita, M. Angew. Chem. Int. Ed. 2009, 48, 3418. (c)

Kawamichi, T.: Kodama, T.; Kawano, M.; Fujita, M. Angew. Chem. Int. Ed. 2008, 47, 8030. (d) Yoshizawa, M.; Fujita, M. Pure Appl. Chem. 2005, 77, 1107. (e) Yoshizawa,

M.; Takeyama, Y.; Okano, T.; Fujita, M. J. Am. Chem. Soc. 2003, 125, 3243.

16. (a) Zhu. J.; Wang, M. J. Macromol. Sci. B, 2008, 47, 401. (b) Chen, Y.-F.; Woo, E.M.; Li, S.-H. Langmuir, 2008, 2ł, 11880.

17. (a) Miura, T.; Mikami. M. Phys. Rev. E. 2007, 75. 031804/(1-12). (b) Mataz, A.; Gregory, B. M.: J. Phys. Condens Mat. 2005, 17, 461.

18. (a) Shin, K.; Obukhov. S.; Chen, J.-T.; Huh, J.; Hwang,Y.; Mok, S.; Dobriyal, P.;

Thiyagarajan, P.; Russell. T. P. Nature Materials, 2007, 6. 961. (b) Clifford L. H.; Lovejeet, S.; Peter J. L. Polym. Mater. Sci. Eng, 2004, 90, 350.

19.(a) Echeverria, C.; Kapral. R.; J. Chem. Phys. 2010, 132, 104902-(1-9). (b) Kalogeras, I. M.; Neagu, E.R. Eur. Phys. J. Part E. 2004, 14, 193.

20. (a) Yorulmaz, M.; Kiraz, A.; Demirel, A. L. J. Phys. Chem. B. 2009, 113, 9640. (b) Ye. H.-M.; Peng, M.; Xu, J.; Guo. B.-H.; Chen, Q.; Yun, T.-L.; Ma, H. Polymer, 2007, 48, 7364. (c) Lu, J.: Mirau, P.A.; Tonelli, A.E. Prog. Polym. Sci. 2002, 27, 357. (d) 
Rallabandi, P.S.; Ford, D. M. J. Membrane Sci. 2000, 171, 239. (e) Alan, E.T. Makromol.

Chem. Macromol. Symp. 1993, 65, 133.

21. Gensel, J.; Liedel, C.; Schoberth, H.G.; Tsarkova, L. Soft Matter, 2009, 5, 2534.

22. Tuteja, B.; Khan, F.; Sundararajan, P. Macromol. Chem. Phys. 2005, 206, 354.

23. (a) Islam, M. R.; Sundararajan, P. R. Can. J. Chem. 2008. 86, 600. (b) Tuteja, B.;

Moniruzzaman, M.; Sundararajan, P. R. Langmuir. 2007, 23, 4709.

24. DRP 276357 (1913), inv.: M. Kardos, Chem. Abstr. 1914, 8, 22317.

25. DRP 276956 (1913), inv.: M. Kardos, Chem. Abstr. 1915, 9, 6398.

26. Roncali, J.; Garnier, F. Appl. Opt. 1984, 23, 2809.

27. Seybold, G.; Wagenblast, G. Dyes Pigm. 1989, 11, 303.

28. Law, K.-Y. Chem. Rev: 1993, 93, 449.

29. Sadrai, M.; Bird, G. R. Opt. Commun. 1984, 51, 62.

30. Sadrai, M.; Hadel. L.; Sauers, R. R.; Husain, S.; Krogh-Jespersen, K.; Westbroo, J.

D.; Bird, G.R. J. Phys. Chem. 1992, 96, 7988.

31. Tang, C.W. Appl. Phys. Let1. 1986, t8, 183.

32. Horowitz, G.; Kouki, F.; Spearman, P.; Fichou, D.: Nogues, C.; Pan, X.; Garnier, F. Adv.Mater. 1996, 8, 242.

33. Suzuki, H.; Hoshino, S. J. Appl. Phys. 1996, 79. 8816. 
34. Wasielewski M. R.; Johnson. D. G.; Niemczyk, M. P.; Gaines „G. L.; O'Neil, M. P.;

Svec, W. A. Advances in Chemistry 1991, 228, 133.

35. Langhals, H. Heterocycles 1995, 40, 477.

36. Nagao, Y. Prog. Org. Coat. 1997, 31, 43.

37. Nagao, Y.; Naito, T.; Abe, Y.; Misono, T. Dyes Pigm. 1996, 32, 71.

38. Nagao, Y.; Misono, T. Bull. Chem. Soc. Jpn. 1981. 5t, 1191.

39. Baby, J.; Asha, S.K. J. Phys. Chem. B 2006, 110, 20937.

40. Kaiser, H.; Lindner, J.: Langhals, H. Chem. Ber. 1991, 124. 529.

41. Tröster. H. Dyes Pigm. 1983, 4, 171.

42. F. (a) Graser, F.; Hädicke, E. Liebigs Ann. ('hem., 1980, 1994. (b) Graser F.; Hadicke, E. Liebigs Ann. Chem., 1984. 483. (c) Hadicke. E.; Graser, F. Acta Crystallogr.. Sect. C, 1986, 42, 189. (d) Hadicke, E.: Graser, F. Acta Crystallogr., Sect. C, 1986, 42, 195. (e)

Klebe, G.; Graser, F.; Hädicke, E.; Berndt, J. Acta Crystallogr., Sect. B, 1989, 45, 69.

43. Zugenmaier, P.: Duff, J.; Bluhm, T. L. Cryst. Res. Technol., 2000. 35, 1095.

44. Wurthner, F. Chem Commun. $2004,14.1564$.

45. Ford, W. E.; Kamat, P. V. J. Phys. Chem., 1987. 9l, 6373.

46. (a) Seybold.G.; Wagenblast. G. Dyes Pigm. 1989, 11, 303. (b) Seybold, G.; Stange.

A. (BASF AG), Ger. Pat., DE 3545 004, 1987 (Chem. Abstr., 1988, 108, 77134. c). 
47. Gvishi, R.; Reisfeld. R.; Burshtein, Z. Chem. Phys. Lett., 1993, $213,338$.

48. Langhals, H.; Demmig, S.; Huber, H. Spectrochim. Acta, 1988, 44 A. 1189.

49. (a) Sui, G.; Orbulescu, J.; Mabrouki, M.; Leblanc, R.M.: Liu, S.; Gregg, B.A. Chem phys chem 2002,3,1041. (b) Chen. Z.; Baumeister, U.; Tschierske, C.; Wurthner, F. Chem. Eur. J. 2007, 13, 450. (c) Brendel, J.; Lu. Y.; Thelakkat, M. J. Mater. Chem., 2010, 20,7255. (d) Wicklein, A.; Lang, A.; Muth. M.A.; Thelakkat, M. J. Am. Chem. Soc. 2009, 131,14442. (e) Wicklein. A.: Lang. A.: Muth, M.A.: J. Am. Chem. Soc. 2009, 131, 14442 .

50. (a) Gould, R. D. Coord. Chem. Rev. 1996. 156, 237. (b) Iwatsu, F. J. Phys. Chem. 1988. 92, 1678. (c) Bayliss, S. M.; Heutz. S.; Rumbles, G.; Jones, T. S. Phys. Chem.Chem. Phys. 1999. 1, 3673.

51. (a) Duzhko, V.; Singer, K. D. J. Phys. Chem. C, 2007, 111, 27. (b) Prince, B.J.;

Williamson, B.E.: Reeves, R. J. J. Lumin. 2001, 93, 293. (c) Ribeiro, A.O.; Biazzotto, J.C.; Serra, O.A. J. Non-Cryst. Solids. 2000, 273, 198. (d) Cook, M. J. J. Mater. Chem. 1996, 6, 677. (e) Philp, D.; Stoddart. J. F. Angew. Chem. Int. Ed. 1996, 35,1154. (f) Lawrence, D. S.; Jiang, T.: Levett, M. Chem. Rev. 1995, 95, 2229.

52. Engel, M.K. in The Porphyrin Handbook, Kadish, K. M.; Smith, K.M.; Guilard, R. Eds, 2003, volume 20, Chapter 122.

53. Chambrier, I.; Cook, M.J.; Helliwell. M.; Powell, A. K. Chem. Commun. 1992,5, 444. 
54. Cook, M.J.; McMurdo, J.; Powell A. K. Chem. Commun. 1993,11. 903.

55. Hammond, R. B.; Roberts, K.J.: Docherty, R.; Edmondson, M.; Gairns, R. J. Chem. Soc., Perkin Trans. 2, 1996, 8. 1527.

56. Chambrier, I.; Cook, M.J.; Wood, P.T. Chem. Commun. 2000, 21, 2133.

57. Bergesen, K.; Haugland, L.; Cook, M. J.: Bryant, G.C. J. Porphyrins Phthalocyanines. 2000, $4,665$.

58. Cai, J.; Wang, J.; Huang, J.; Chen. N. Chinese Sci. Bull. 2002, 47. 644.

59. Wang, J.; Huang, J.; Cai, J.; Chen, N. Chinese. J. Struct. Chem. 2002. 21, 617.

60. Gao, Y.; Chen, Y.; Li, R.; Bian, Y.; Li, X.; Jiang, J. Chem. Eur. J. 2009, 15, 13241

61. (a) Ajaygosh. A.; George, S. J.; Praveen, V. K. Angew. Chem., Int. Ed. 2003, 42, 332.

(b) Urabe, H.; Jung, J. H.; Ono, Y.; Shinkai, S.; Soai, K. Tetrahedron Let1. 2003, 47. 721.

(c) Tiller, J. C. Angew. Chem., Int. Ed. 2003, 42, 3072. (d) Friggeri. A.; Feringa, B.; van

Esch, J. J. Controlled Release 2004, 97, 241. (e) Roubeau, O.; Colin, A.; Schmitt, V.;

Clerac, R. Angew. Chem. Int. Ed 2004, 43, 3283. (f) Yang. Z.; Gu, H.; Fu, D.; Gao, P.;

Lam, J. K.; Xu, B. Adv. Mater. 2004, 16. 1440. (g) Sugiyasu, K.; Fujita, N.: Shinkai, S. Angew. Chem., Int. Ed. 2004, 43, 1229. (h) Yao, S.; Begnin, U.; Gress, T.; Lysetska, M.; Wurthner, F. J. Am. Chem. Soc. 2004, 126, 8336. (i) Kiyonaza, S.; Sada, K.; Yoshimura, I.; Shinkai, S.; Kato. N.; Hamachi, I. Nat. Mater. 2004, 3, 58. (j) Henisch. H. K. Crystal 
Growth in Gels; Pennsylvania State University Press: University Park, PA, 1970. (k)

Srivastava, S. P.; Saxena, A. K.: Tendon, R. S.: Shekhar, V. Fuel 1997, 76, 625.

62. Steichele, K. Belg. Patent 882,922, 1980. Chem. Abstr. 1980, 94, 66768d.

63. Saka, K.; Noda, K. Jpn. Kokai Tokkyo Koho JP 62179 584, 1987; Chem. Abstr. 1987, 108, 39820r.

64. Tanaka, K.; Kano, Y.; Yoshida. K. Chem. Abstr. 1988, 110, 79137w.

65. Kinoshita, H.; Sekiya. M.; Mishima, M. Eur. Pat. EP 274 756, 1988; Chem. Abstr. 1988, 109, 233979k.

66. Kochansky, J.; Cohen, C. F. J. Agric. Ent. 1990. 7, 293.

67. Jpn. Kokai Tokkyo Koho JP 58201 758, 1983; Chem. Abstr. 1983, 100, 174289z.

68. Tanaka, T.; Yoshitomi, T.; Hanada, Y.; Ohashi, M.; Takeda, Y. Chem. Abstr. $1985,107,20896 b$.

69. Goodbrand, B.; Boils, D.; Sundararajan. P. R.; Wong, R.; Malhotra, S. U. S. Patent 6,187,082, 2001.

70. (a) Moniruzzaman, M.; Sundararajan. P. R. Langmuir 2005, 21, 3802. (b) Khanna, S.; Moniruzzaman, M.; Sundararajan. P. R. J. Phys. Chem Part B 2006, 110, 15251. (c) Khanna, S.; Khan, M. K.; Sundararajan, P. Langmuir 2009, 25, 13183. (d) Khan, M. K.; Sundararajan, P. R. J. Phys. Chem. B 2008, 112, 4223. (e) Khan, M. K.; Sundararajan, P. R. Chem. Eur. J. 2010, 17, 1184. 
71. (a) Jelly, E. E. Nature 1936, 138. 1009. (b) Scheibe, G. Angew. Chem. 1936, 49, 563.

(c) Mishra, A.; Behera, R. K.; Behera P. K.; Mishra. B. K.; Behera, G. B. Chem. Rev. 2000, 100. 1973.

72. Spano, F.C. Acc. Chem. Res. $2010,43,429$.

73. (a) Askew, B.; Ballester, P.; Buhr, C.; Jeong, K. S.; Jones. S.; Parris, K.; Williams. K.; Rebek, J., Jr. J. Am. Chem. Soc. 1989, 111 .1082. (b) Zimmerman, S. C.: VanZyl, C. M.; Hamilton, G. S. J. Am. Chem. Soc. 1989, 111,1373. (c) Schneider. H.-J.; Philippi, K.; Pohlmann, J. Angew. Chem. Int. Ed. 1984, 23. 908. (d) Canceill, J.; Lacombe, L.; Collet, A. Chem. Commun. 1987, 3, 219. (e) Muehldorf, A. V.; Van Engen, D.; Warner, J. C.; Hamilton, A. D. J. Am. Chem. Soc. 1988, 110, 6561.

74. (a) Langlet, J.; Claverie, P.; Caron, F.: Boeuve, J. C. Int. J. Quantum Chem. 1981, 19, 299. (b) Price, S. L.; Stone, A. J. J. Chem. Phys. 1987, 86, 2859. (c) Hunter, C. A.; Sanders, J. K. M. J. Am. Chem. Soc. 1990, 112, 5525. 


\section{Chapter II Experimental}




\section{1 Introduction}

Self assembly as a process of association creates different morphologies which often show new and improved property than that of the single molecule. The self assembly of a system can be tuned by incorporating structural change or by solvents, temperature etc. These changes can be assessed by using a combination of experimental techniques such as Differential Scanning Calorimetry (DSC), X-ray Diffraction (XRD), Optical Microscopy (OM), Scanning Electron Microscopy (SEM) and Dynamic Light Scattering (DLS). This chapter describes these techniques, used in this thesis to study the morphological and thermal behavior of different organic functional molecules by itself and in polymer matrix. It also discusses the synthesis of these compounds used here. In this study, the characterization of the synthesized products in different intermediate or final steps was carried out by FTIR and ${ }^{1}$ HNMR spectroscopy. UV-Vis spectroscopy and DSC. The terms used in the morphology along with the concepts related in self assembly have also been described in brief.

\section{2 Instruments and Methods}

\section{2. 1 FTIR Spectroscopy}

Infrared (IR) spectroscopy is an extremely powerful analytical technique for both qualitative (fingerprinting of compounds; recognition of specific chemical bonds, or groups; gross structural features) and quantitative (measurements of reaction rates, determination of thermodynamic data, process and control) analysis. The underlying basis of applied IR spectroscopy is the fact that practically all organic compounds possess selective absorption at certain frequencies in the IR portion of the electromagnetic 
spectrum due to its vibrational degrees of freedom ${ }^{1.2}$. Molecular vibration with a permanent dipole moment creates a fluctuation of dipole moment which can be considered as a wave of electric dipole moment which interacts with the electromagnetic radiation. Organic applications of infrared spectroscopy are almost entirely concerned with the range of $650-4000 \mathrm{~cm}^{-1}$. The region of frequencies below $650 \mathrm{~cm}^{-1}$ is called the far infrared whereas the region above $4000 \mathrm{~cm}^{-1}$ is known as near infrared. The information regarding the functional group present in a molecule is obtained from the region between $1400-4000 \mathrm{~cm}^{-1}$. The region from $600-1400 \mathrm{~cm}^{-1}$ is known as fingerprint region because the pattern of absorption in this region is unique to any particular compound. ${ }^{3,4}$ A plot of transmission or absorption values versus frequency or wavelength units constitutes an IR spectrum, which is a characteristic of the sample being studied.

The absorption of infrared is a quantized process. The bonds between the atoms in a molecule vibrate with certain energy. When the molecules are exposed to the IR radiation, the frequencies of the radiation, which match the natural vibrational frequencies of the molecules, get absorbed. All the bonds cannot absorb IR radiation. To absorb radiation, bonds should have permanent dipole moments. A change in a dipole moment takes place with a change in the position of the centers of positive and negative charges due to the atomic motions. ${ }^{5-7}$ This changing electrical dipole moment of the bond then interacts with the changing electromagnetic field of the incoming radiation. The matching frequency is absorbed and this absorbed energy increases the amplitude of the vibrational motion of the bonds and the molecules are excited to a higher energy state. This gives rise to the absorption bands appearing on the spectrogram. Basically there are 
two different modes of vibrations in a molecule which are infrared active i.e. stretching and bending. Stretching vibrations can be symmetric or asymmetric while bending can be classified as scissoring, wagging, rocking. and twisting.

Michelson M129 BOMEN Fourier Transform Infrared (FTIR) spectrometer was used for the present studies. The experiments were run at ambient conditions. Data were collected using BOMEN GRAMS / 386 software. In some cases, the FTIR spectra of the samples were recorded in the form of transparent $\mathrm{KBr}$ pellets. A background FTIR spectrum was taken for each experiment with an identical sample holder. In some cases, pure samples or chloroform solution of the samples was drop cast on $\mathrm{NaCl}$ discs, dried and IR was taken.

\subsubsection{UV-visible Spectroscopy}

The UV-Visible spectrum corresponds to electronic excitation (figure 2.1) and the energy levels depend on the chemical bonds within the specimen. ${ }^{7}$ Sigma-electrons $(\sigma)$, involved in covalent bonds, absorb high energy photons in the UV region, where $\pi$ electrons absorb low energy photos at longer wavelengths, often in the visible region (figure 2.1). Although UV-visible spectroscopy is not capable alone of completely identifying an unknown compound, the advantage of using very small samples and rapid analysis makes it still a common routine analytical technique. One of the most useful applications of UV-visible spectroscopy to polymers is the measurement of the length of conjugation in unsaturated molecules. 

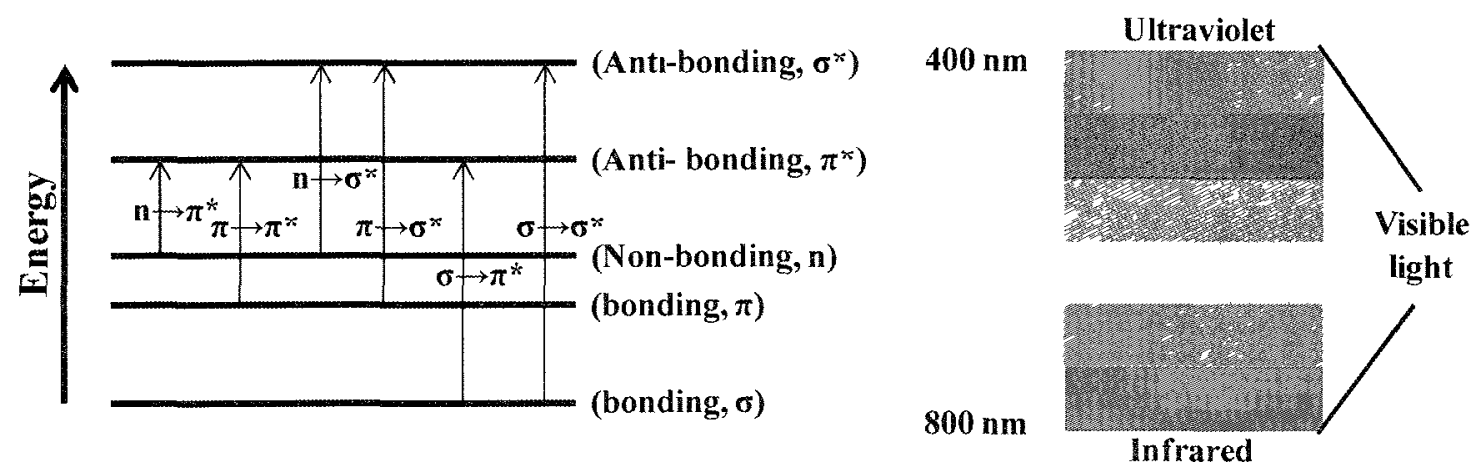

Figure 2.1: Energy levels.

A modern application is in the analysis of rigid rod molecules with liquid crystal properties. The stiffness of the molecule derives from the presence of cyclic structures along the backbone. The uninterrupted conjugation lengths can be obtained using UVvisible spectroscopy. The $\pi$-electrons in a conjugated system are delocalized, which means that they are not restricted to an atomic orbit, but can move freely within the conjugated region. The theory starts with the proposal that the behavior of a $\pi$-electron can be modeled by a particle in a square potential well with infinite side and whose length is chosen to coincide with the conjugation length within the molecule. Such a particle can exit anywhere within the well, but it cannot escape. This represents a good approximation because a $\pi$-electron has a very low probability of escape. Another application of the measurement of the conjugation length is in the study of cis-trans isomers. The other application is in the analysis of copolymers. If the two (or more) monomers process characteristic absorptions that do not overlap. then the relative intensities of the absorption measured on the copolymer formed from them can be used to estimate composition. 
The UV-Vis spectroscopic measurements were performed at ambient conditions using a Varian CARY 3 UV-Vis spectrophotometer. The path length of the quartz sample cube was $10 \mathrm{~mm}$. Temperature dependent UV-Vis spectra were recorded with a CARY 300 Bio UV-Vis spectrophotometer with a Varian temperature controller (operating temperature $-10^{\circ} \mathrm{C}$ to $100^{\circ} \mathrm{C}$ ). The data were processed by CARY WinUV Software version 3.00 .

\subsubsection{Fluorescence Spectroscopy}

Fluorescence occurs when some chromophores, after being electronically excited, reemit the energy. During the absorption of the UV photon, the chromophore is usually brought to a state that is excited both electronically and vibrationally. However, nonradiative relaxation very quickly brings the chromophore to its lowest vibronic level within the electronically excited state. When the remaining excitation energy is reemitted and the chromophore returns to the electronic ground state, the molecule usually retains some vibrational energy (which is subsequently also dissipated). Thus, the fluorescent radiation occurs at longer wavelengths than the exciting radiation; a whole fluorescence spectrum is observed because the molecules may end up in different vibronic states (figure 2.2). The re-radiation process is slow as electronic phenomena goes. The lifetimes of the excited states are measured in nanoseconds, while most transitions among electronic states are completed in picoseconds or faster. In macromolecular studies, the chromophore is either a part of the macromolecule itself or fluorescent probes are covalently attached to the macromolecules (e.g., on chain end). 
Since most fluorescence occurs in solution, the environmental factors must be considered. ${ }^{8}$ These factors include interactions with solvent and other dissolved compounds, temperature, $\mathrm{pH}$, and the concentration of fluorescent species. The effects that these four parameters have upon fluorescence vary from fluorescent moiety to moiety. Both absorption and emission spectra as well as the quantum efficiencies of fluorescent molecules are influenced by the above mentioned parameters. Normally absorption and fluorescence spectra are mirror images to each other.

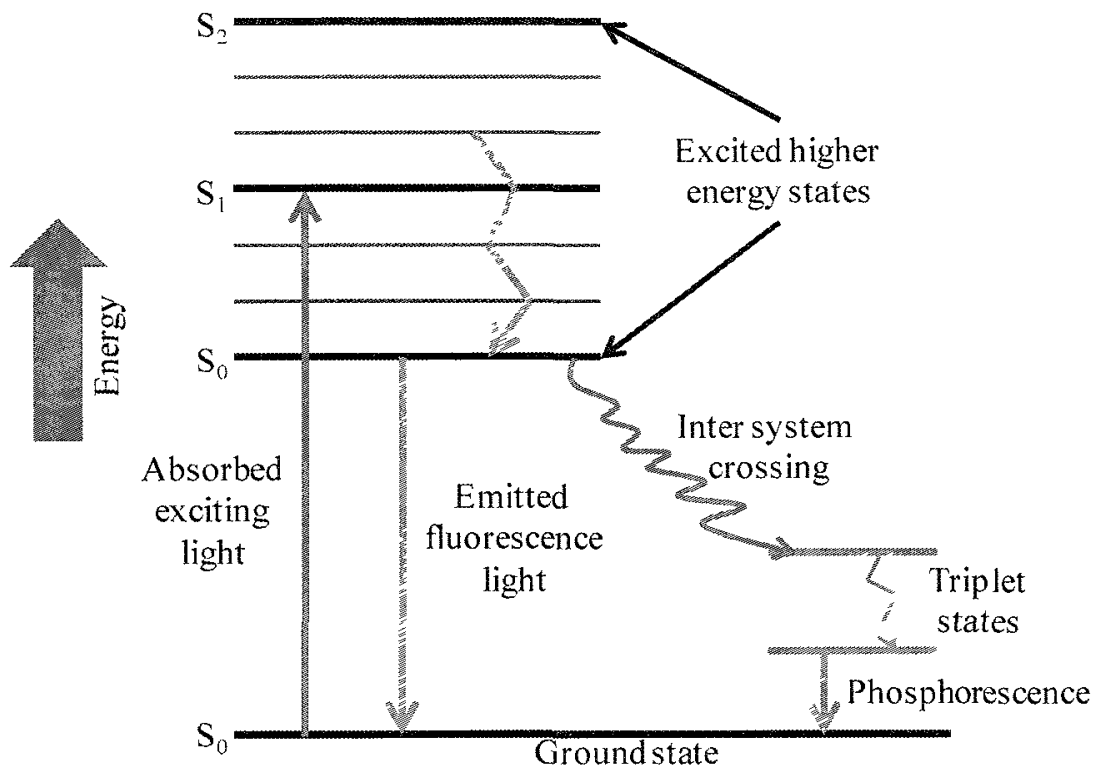

Figure 2.2: Jablonski diagram.

Fluorescent Spectra were recorded with a Varian CARY Fluorescent spectrophotometer at $\lambda_{\mathrm{ex}} 460 \mathrm{~nm}$ and $417 \mathrm{~nm}$ with a bandwidth of $5 \mathrm{~nm}$ for excitation and $5 \mathrm{~nm}$ for emission. Data collection and processing were done by Eclipse WinFLR Software (version 1.1). 


\subsubsection{NMR Spectroscopy:}

Over the past fifty years nuclear magnetic resonance spectroscopy, commonly referred to as NMR, has become the preeminent technique for determining the structure of organic compounds. ${ }^{5}$ Of all the spectroscopic methods, it is the only one for which a complete analysis and interpretation of the entire spectrum is normally expected. Although larger amounts of sample are needed than for mass spectroscopy, nmr is non-destructive, and with modern instruments good data may be obtained from samples weighing less than a milligram. To be successful in using $\mathrm{nmr}$ as an analytical tool, it is necessary to understand the physical principles on which the methods are based.

The nuclei of many elemental isotopes have a characteristic spin (I). Some nuclei have integral spins (e.g. $I=1,2,3 \ldots$ ), some have fractional spins (e.g. $I=1 / 2.3 / 2,5 / 2$ ....), and a few have no spin, $\mathrm{I}=0$ (e.g. ${ }^{12} \mathrm{C},{ }^{16} \mathrm{O},{ }^{32} \mathrm{~S}$ etc.). Isotopes of particular interest and use to organic chemists are ${ }^{1} \mathrm{H},{ }^{13} \mathrm{C},{ }^{19} \mathrm{~F}$ and ${ }^{31} \mathrm{P}$, all of which have $\mathrm{I}=1 / 2$. Since the analysis of this spin state is fairly straightforeward, our discussion of nmr will be limited to these and other $I=1 / 2$ nuclei. An element with fractional spin generates a magnetic field which magnetic moment is proportional to the spin. With the presence of an external magnet split the energy level to two spin states which are $+1 / 2$ and $-1 / 2$ parallel and anti parallel to the magnetic field respectively separated by an energy gap proportional to the external magnetic field. Transition between these spin states leads to absorption or emission of an energy. Irradiation of a sample with radio frequency ( $\mathrm{rf}$ ) energy corresponding exactly to the spin state separation of a specific set of nuclei will cause excitation of those nuclei in the $+1 / 2$ state to the higher $-1 / 2$ spin state. The energy change 
is observed as a resonance peak or line which is depends on external magnetic field strength, temperature etc. The position of the resonance line or peak tells the nature, location and conformation of the atomic groups in the vicinity of the protons. The change in the resonance, called chemical shift, is measured in parts per million in frequencies on a scale labeled $\delta$. The zero of the $\delta$ scale in a substance is a reference point provided by the single resonance of the equivalent protons in a substance showing minimum chemical, such as tetramethyl silane (TMS).

NMR technique can be used more additional information about the complex bonding, geometry using the coupling of the resonance of protons with adjacent carbon resulting the splitting of the resonance peaks or lines into $n+1$ multiplet, where $n$ is the number of equivalent neighboring protons.

In this thesis, a Bruker- 400 instrument was used to record the ${ }^{1}$ HNMR spectra at room temperature and at different temperatures above room temperature. Tetramethyl silane was used as an internal standard.

\subsubsection{Differential Scanning Calorimetry (DSC):}

Differential Scanning Calorimetry is a technique that is used very often for quantitative studies of thermal transitions in polymers, metals. ceramics, composites and organic materials. In this technique, the sample and an inert reference are heated, usually in a nitrogen atmosphere, and thermal transitions in the sample are detected and measured. 
An average-temperature circuit measures and controls the temperature of the sample and sample holders to conform to a predetermined time-temperature program. At the same time, a temperature-difference circuit compares the temperatures of the sample and reference holder and proportions power to the heater in each holder so that the temperature remains equal (figure 2.3). When the sample undergoes a thermal transition, the power to the two heaters is adjusted to maintain their temperatures. The temperature is plotted along one axis of a $x-y$ recorder and a signal proportional to the power difference is plotted along the second axis of the recorder (figure 2.4). This gives valuable information about the physical or chemical properties of the materials.

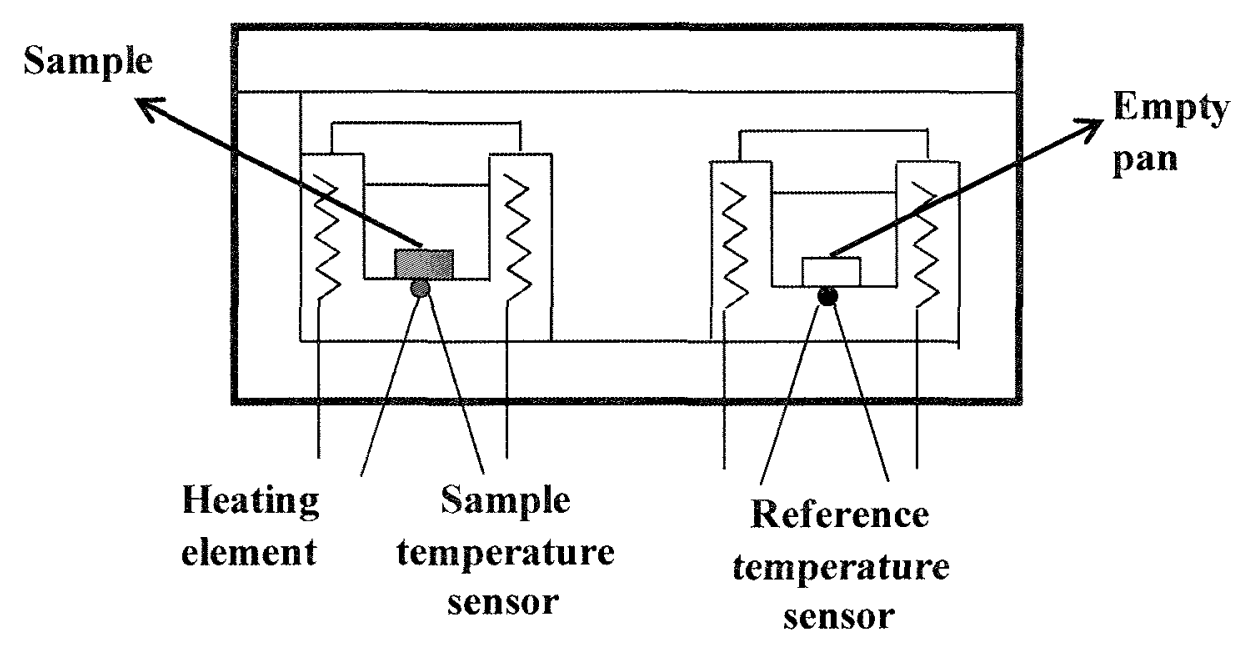

Figure 2.3: Schematic representation of Differential Scanning Calorimeter.

The area under the resulting curve is a direct measure of the heat of transition. The applications of DSC include determination of melting temperature $\left(T_{m}\right)$, the crystallization temperature $\left(T_{c}\right)$, glass transition temperatures $\left(T_{g}\right)$ and the specific heat capacity $(C p)$ of the materials. Liquid crystalline and polymorpohic transition can be detected from DSC as 
well. ${ }^{9.10}$ The heat flow is going to be shown in units of heat, $q$ supplied per unit time, $t$. The heating rate is temperature increase $T$ per unit time, $t$.

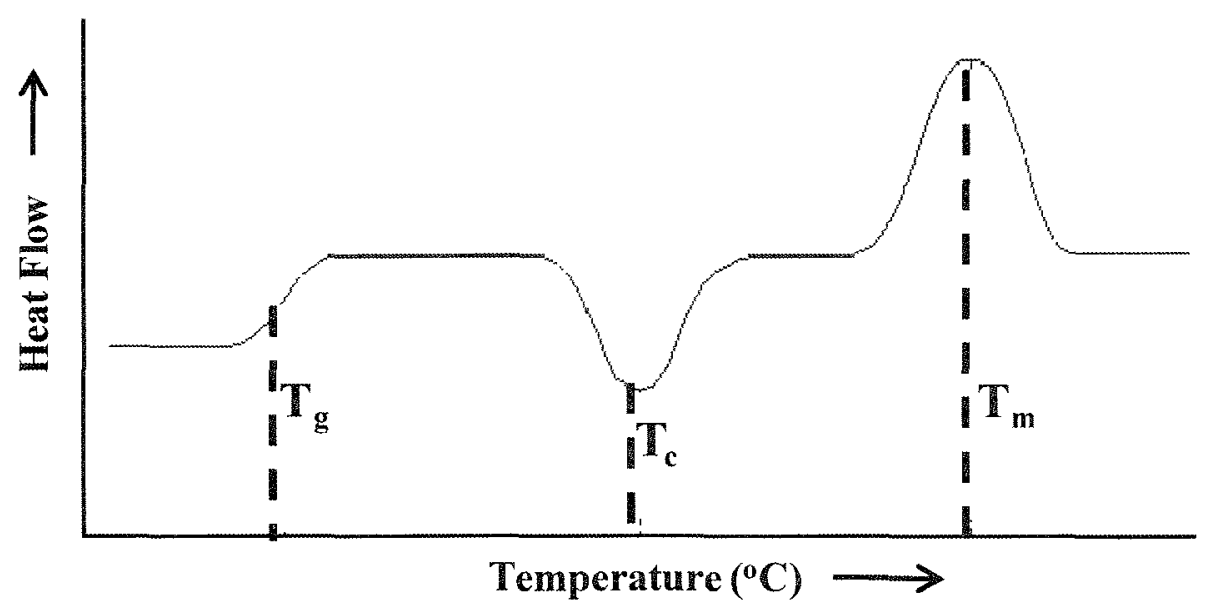

Figure 2.4: A typical DSC plot.

In this study, thermal analysis was performed using a TA instrument 2010 differential scanning calorimeter at a heating rate of $10^{\circ} \mathrm{C} /$ minute. The instrument was calibrated for temperature and energy with indium and tin reference samples. DSC traces were recorded in a nitrogen atmosphere. The uncertainty was $\pm 1{ }^{\circ} \mathrm{C}$ for the melting point and $\pm 2 \mathrm{~J} / \mathrm{g}$ for the heat of fusion. DSC can also tell us how much of a polymer is crystalline and how much is amorphous.

\subsubsection{Optical Microscopy (OM)}

Optical microscopy is the most widely used easy and convenient technique in the field of material science to study the surface and the bulk morphology which uses light and lenses to magnify the images of small sample to a large one. The optical components of a modern microscope are very complex and for a microscope to work well, the whole 
optical path has to be very accurately set up and controlled. Despite this, the basic optical principles of a microscope are quite simple. ${ }^{10,12}$

The objective lens is, at its simplest, a very high powered magnifying glass i.e. a lens with a very short focal length. This is brought very close to the specimen being examined so that the light from the specimen comes to a focus about $160 \mathrm{~mm}$ inside the microscope tube. This creates an enlarged image of the subject. This image is inverted and can be seen by removing the eyepiece and placing a piece of tracing paper over the end of the tube. By carefully focusing a brightly lit specimen, a highly enlarged image can be seen. It is this real image that is viewed by the eyepiece lens that provides further enlargement.

In most microscopes, the eyepiece is a compound lens, with one component lens near the front and one near the back of the eyepiece tube. This forms an air-separated couplet. In many designs, the virtual image comes to a focus between the two lenses of the eyepiece, the first lens bringing the real image to a focus and the second lens enabling the eye to focus on the virtual image.

The optical microscope with polarized light in the transmission mode and reflection mode was used to study the optical characteristics of the all the samples. In a crosspolarized setting the polarizer and analyzer are placed in such a way that their orientation axes are perpendicular to each other. 
A Zesis Axioplan polarised optical microscope (OM) was used for recording the optical micrographs of all the cases. The calibration of the microscope was done with a micro scale with $10 \mu \mathrm{m}$ division. Films were cut and attached with microscopic slides before taking the OM images. For the solution sample drop cast samples were used as such or dried on glass slide and used for taking the images. For hot stage experiment, a Linkam hot stage LTS 350 was used, with a Linkam TMS 94 controller. The samples were heated to a temperature $5-10{ }^{\circ} \mathrm{C}$ higher than their melting point, at the rate of $5{ }^{\circ} \mathrm{C}$ /minute and held there isothermally for about 10 minutes. They were then cooled at the rate of $0.5^{\circ} \mathrm{C}$ /minute up to the room temperature. Northern Eclipse (version 6.0) image processing software was used to capture the images and calculate the size of the crystals.

\subsubsection{Scanning Electron Microscopy (SEM)}

The scanning electron microscope (SEM) is a type of electron microscope that images the sample surface by scanning it with a high-energy beam of electrons. The electrons interact with the atoms that make up the sample producing signals that contain information about the sample's surface topography, composition and other properties. ${ }^{10,13}$ The types of signals produced by an SEM include secondary electrons, back-scattered electrons (BSE), characteristic X-rays, light (cathodoluminescence), specimen current and transmitted electrons. Secondary electron detectors are common in all SEMs.

The scanning electron microscope uses a high-energy electron beam obtained from the emission of tungsten filament, to form images. This beam is passed through a series of lenses and scanning coils, which place a small spot of electrons on the specimen. 
These electrons penetrate the surface and produce secondary electrons. Secondary electrons get liberated from the specimen surface and the detector collects these electrons and sends the signals to an amplifier and the image is recorded. The SEM can produce three-dimensional images of high resolution, which means that closely spaced features can be examined at a high magnification. ${ }^{14-16}$ SEM provides useful information on surface topology with a resolution of about $100 \AA$. The combination of large depth of focus, greater resolution, and higher magnification makes the SEM a very useful instrument in research areas today.

SEM images were obtained either by using a JEOL JSM-6400 scanning electron microscope or by Tesean Vega-II XMU VPSEM. The samples were coated with 60:40 $\mathrm{Au} / \mathrm{Pd}$ using a sputter coater (Hummer VII model by Anatech Ltd.). They were then placed inside the SEM under vacuum. The accelerating voltage of the beam was $20 \mathrm{kV}$.

\subsubsection{Dynamic Light Scattering (DLS):}

Dynamic light scattering (DLS), sometimes referred to as Photon Correlation Spectroscopy (PCS) or Quasi-Elastic Light Scattering (QELS), is a non-invasive, wellestablished technique for measuring the size of molecules and particles typically in the submicron region, and with the latest technology lower than 1 nanometer. Particles, emulsions and molecules in suspension undergo Brownian motion. This is the motion induced by the bombardment by solvent molecules that themselves are moving due to their thermal energy. 
If the particles or molecules are illuminated with a laser, the intensity of the scattered light fluctuates at a rate that is dependent upon the size of the particles as smaller particles are "kicked" further by the solvent molecules and move more rapidly. Analysis of these intensity fluctuations yields the velocity of the Brownian motion and hence the particle size using the Stokes-Einstein relationship.

The diameter that is measured in Dynamic Light Scattering is called the hydrodynamic diameter and refers to how a particle diffuses within a fluid. The diameter obtained by this technique is that of a sphere that has the same translational diffusion coefficient as the particle being measured.

The translational diffusion coefficient will depend not only on the size of the particle "core", but also on any surface structure, as well as the concentration and type of ions in the medium. This means that the size can be larger than measured by electron microscopy, for example, where the particle is removed from its native environment.

Particle size distributions can be calculated either assuming some standard form such as log-normal or without any such assumption. In the latter case. it becomes possible, within certain limitations, to characterize multimodal or skewed distributions. The size range for which dynamic light scattering is appropriate is typically submicron with some capability to deal with particles up to a few microns in diameter. The lower limit of particle size depends on the scattering properties of the particles concerned (relative refractive 
index of particle and medium), incident light intensity (laser power and wavelength) and detector / optics configuration.

The Dynamic Light Scattering (DLS) experiment was done with a NanoDLS particle size analyzer (Brookhaven Instruments Corp) at $25^{\circ} \mathrm{C}$. The wavelength of the laser was set at $637 \mathrm{~nm}$. The machine was calibrated with standard polystyrene nanoparticle of $90 \mathrm{~nm}$ diameter. The effective diameter is an average of 10 runs each time. The solutions were made in water at $10^{-3} \mathrm{M}$ concentration. Effective diameter was calculated using the NanoDLS Particle Sizing Software Ver. 4.11.

\subsubsection{X-ray Diffraction (XRD):}

$\mathrm{X}$-ray diffraction method is a very powerful technique to get the information about the orderly arrangement of the atoms or molecules in the crystal structure by the interaction of strong electromagnetic radiation to give interference with the structures comparable to the size of the wavelength of the radiation (Figure 2.5). ${ }^{13}$

A crystal is a complex, although orderly, arrangement of atoms. All atoms in the path of an X-ray beam scatter X-rays simultaneously. In general, the scattered waves interfere with and destroy one another, but in certain specific directions they combine to form new wave fronts. This cooperative scattering is known as diffraction. The directions of the possible diffraction depend only on the size and shape of the unit cell. 
Complete information on the molecular ordering in a crystalline or semi-crystalline material can be obtained from a single crystal by single crystal diffraction. The diffraction patterns at all possible angles are recorded by rotating the crystal about an axis perpendicular to the incident beam. Crystallographic information can be obtained from a powder sample as well without single crystal. In that case a powder X-ray diffractometer can be used. Valuable information can be extracted by using this instrument, such as interplanar distances between atomic planes ( $d$-spacing), crystallite size and percent crystallinity. In powder X-ray diffraction, the reflections are obtained from several microcrystals with random orientation.

The basic equation of X-ray diffraction is the Bragg formula:

$$
2 \mathrm{~d} \sin \theta=n \lambda
$$

Where $\mathrm{d}$, is the distance between the atomic planes, $\theta$ the angle of incidence of the $\mathrm{x}$-ray beam on the atomic planes, $\lambda$ the wavelength of $\mathrm{x}$-ray radiation and $\mathrm{n}(1,2, \cdots, \mathrm{n})$ is the order of reflections. 


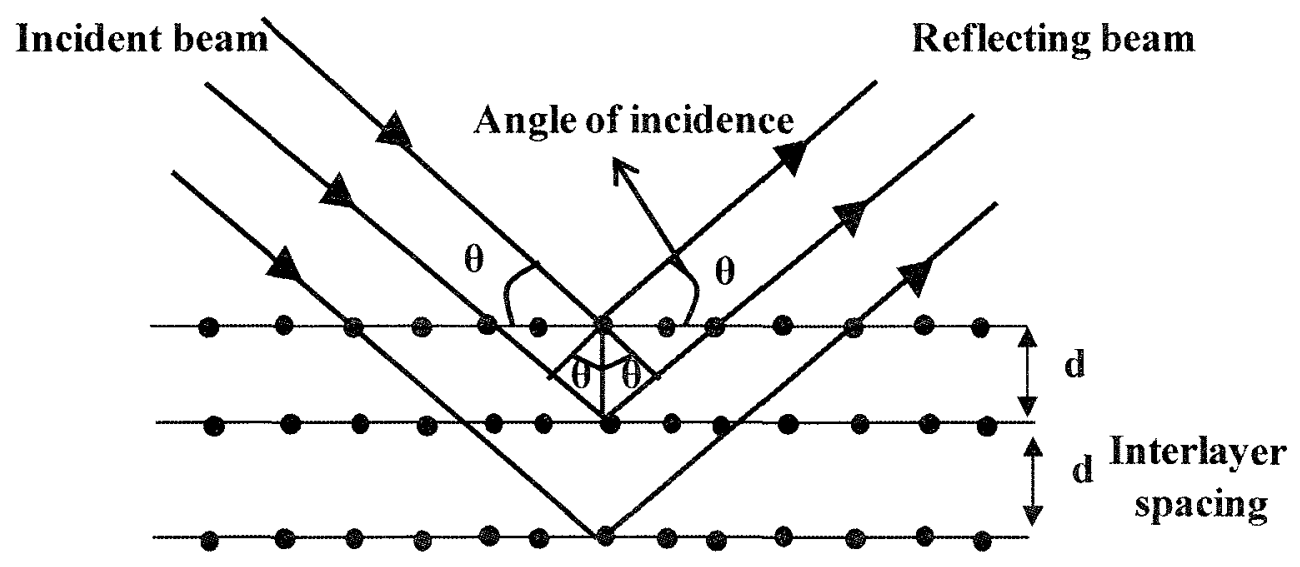

Figure 2.5: Principle of diffraction. Figure shows a plane of beam of parallel x-rays penetrating a stack of planes of spacing $d$, at a glancing angle of incidence $\theta$. Each plane must be pictured as reflecting a fraction of the incident beam. ${ }^{9}$

Rearrangement of Bragg's equation gives the d-spacing as:

$$
d=\lambda / 2 \sin \theta
$$

We see an inverse relation between the interlayer distance and scattering angle. The above equation (2.2) shows that when the distance between the two planes in the lattice is

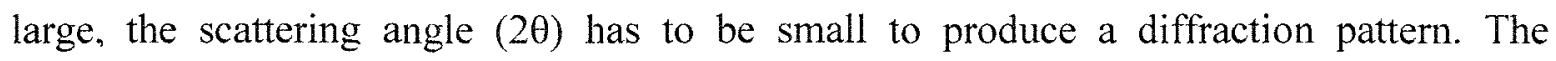
diffraction obtained at angles smaller than $2^{\circ}(d>\approx 50 \AA)$ is called Small Angle X-ray Scattering (SAXS). ${ }^{17}$ The diffraction obtained at all larger angles, theoretically extending up to $180^{\circ}$ is called Wide Angle X-ray Diffraction (WAXD). WAXD provides information about the spatial arrangement of the atoms, whereas, SAXS provides the information about domain arrangement (e.g. long spacing). 


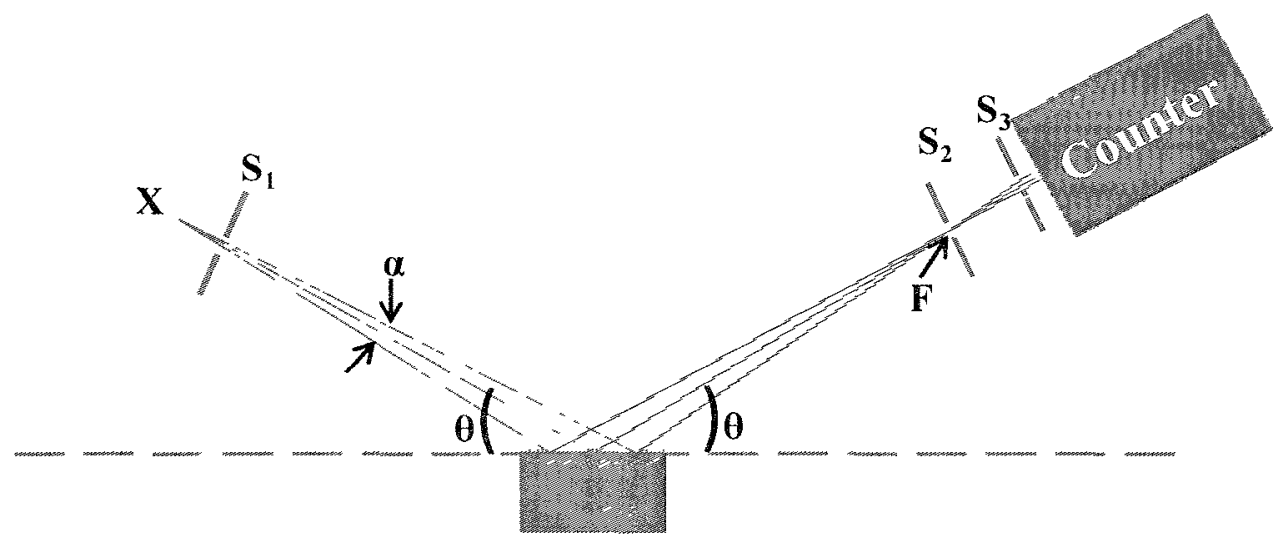

Figure 2.6: Schematic of the Bragg diffraction with the powder X-ray diffractometer used for the present study [redrawn from Alexander ${ }^{17}$ ]. Here, $S_{1}, S_{2}$ and $S_{3}$ are the divergence slit, receiving slit and scatter slit respectively.

In the present work, a Philips automated powder diffractometer (PW 1710) was used for X-ray diffraction studies with $\mathrm{Ni-filtered} \mathrm{Cu} \mathrm{K} \mathrm{K}_{\alpha}$ radiation $(\lambda=1.5418 \AA)$. Normally samples were put onto the glass slides or x-ray glass holder, pressed with second glass slide slightly for the X-ray diffraction. Diffraction data were recorded in the range of $2^{\circ} \leq 2 \theta \leq 50^{\circ}$, using the software MDI Datascan 3.2 (Materials Data Inc.. Livermore $\mathrm{CA}$ ). The possible absence of the reflection in some direction was checked by taking additional diffractograms with the sample turned in the plane of measurement by $90^{\circ}$. The results were analyzed using the MDI Jade 5.0 XRD pattern processing software. $\mathrm{X}$-ray scans were recorded at room temperature. 


\subsection{References}

1. Herzberg. G. Molecular Spectra and Molecular Structure, $2^{\text {nd }}$ ed., van Nostrand Reinhold Company: New York, 1950.

2. Steinfeld. J.I. Molecules and Radiation, An Introduction to Molecular Spectroscopy; The MIT Press: Cambridge, 1985.

3. Kalsi, P.S. Spectroscopy of Organic Chemistry. New Age International Ltd, 4th Ed., 2000, page 60-63.

4. Pavia, D.L.; Lampman, G.M.; Kriz, G.S. Introduction to Spectroscopy, Harcourt College Publisher, 3rd Ed., 2001, page 41.

5. Mirau, P.A. in Polymer Characterisation, Hunt, B.J.; James, M.I. (Eds), Blackie A \& P: Glasgow, 1993, Chapter 3.

6. Kemp, W. Organic spectroscopy, $3^{\text {rd }}$ ed., Macmillan Education Ltd.: London. 1991.

7. Rao, C.N.R. Ultra-Violet and Visible Spectroscopy: Chemical Applications, 3th Ed.. Butterworths: London. 1975, page 100.

8. Pesce, A.M.; Rosen, C.-G.; Pasby, T.L. Fluorescence Spectroscopy, Marcel Dekker Inc: New York, 1971.

9. Wendlandt, W. W. Thermal Analysis, Wiley Interscience: New York, 1986, page 667.

10. Stevens, M.P. Polymer Chemistry: an Introduction, $3^{\text {rd }}$ ed., Oxford University Press: New York, 1999. Chap. 5.

11. (a) Fox, T.G.; Flory, P.J. J. Appl. Phys. 1950, 21, 581. (b) Fox, T.G.; Flory, P.J. J. Polym. Sci. 1954, 17, 315.

12. Saville, B. P. In Applied Polymer Light Microscopy: Hemsley, D.A., Ed.; Elsevier Science Publishers Ltd.: New York, 1989, Chap. 4. 
13. Billmeyer, F.W. Textbook of Polymer Science, $3^{\text {rd }}$ ed., Wiley- Interscience: New York, 1984, Chap. 9.

14. Curson. A. D. Polymer Light Microscopy. Hemsley, D.A., Ed.; Elsevier Science Publishers Ltd.: New York, 1989, Chap 1.

15. Thornton, P.R. Scanning Electron Microscopy, Applications 10 Materials and Device Science, Chapman and Hall Ltd.: London. 1968, Chap. 2.

16. Temple. B. J. In Principles and Techniques of Scanning Electron Microscopy, Hayat, M.A., Ed.; Van Nostrand Reinhold Comp.: New York, 1974. Chap. 1.

17. Alexander, L.E. X-Ray Diffraction Methods in Polymer Science, Wiley-Interscience: New York, 1969. 


\section{Chapter III}

Self-assembly of Rod-Coil and Coil-Rod-Coil Amphiphilic Polymers Based on Perylene Tetracarboxylic Diimide in Water, Mixed Solvents and in Bulk.

Submitted to Chem. Eur. J. 2010(accepted). 


\subsection{Introduction}

Interest in rod-coil polymers has been evolving significantly during the past few years. ${ }^{1-11}$ These amphiphilic or solvent-selective block copolymers present various types of self-assembled morphologies, which result from the packing of the rod segments, either as crystalline or liquid crystalline structures. The large vesicular and spherical morphologies arising from these polymersomes have been considered for drug delivery and similar applications. Typically the rod segment consists of a helical polymer such as polypeptides or a liquid crystalline mesogen. A number of polymers which could qualify for such a rod segment have been reviewed. ${ }^{4}$ The area of rod-coil polymers evolved following studies on self-assembly and morphology of amphiphilic block copolymers which began a decade earlier. ${ }^{12-14}$ The blocks usually were of flexible or semi-flexible coil-coil type, with asymmetric block lengths, and one of the blocks being hydrophilic. By varying the relative lengths of the blocks (as in the case of e.g., polystyrene-copolyacrylic acid), as well as the polarity of the aqueous medium. several types of morphologies such as spheres, rods, vesicles. and lamellae have been observed. ${ }^{12 b .}$ 14a.c Thus such block copolymers are a means to create designer morphologies.

Organic chromophores, such as derivatives of perylene, phthalocyanine and porphyrin have been the focus of significant scientific interest due to their use in photoreceptors $^{15}$, field effect transistors ${ }^{15}$, as well as storage of optical data. ${ }^{16}$ Their semiconductor properties also find applications in solar cells and photovoltaic cells. ${ }^{17}$ These functional properties depend on the molecular aggregation of these chromophores, which enable the efficient conduction of electrons or holes within the aggregates and the 
properties of the semiconductor generally improve with increasing structural order. ${ }^{18}$ While the $\pi$ interactions enable the self-assembly of these chromophores, the nature of the assembly can be modulated by substitution of side groups. Most of the reported perylenediimides use short side groups. ${ }^{19}$ Short linear side groups give rise to liquid crystalline properties and crystallization results in morphologies consisting of nanowires. Two or three radiating side groups lead to discotic liquid crystalline assemblies or dumbbell or conical morphologies. ${ }^{19 c, d}$ Perylenebisimide-alkyl chain alternating copolymers (with $\mathrm{C}_{4}$ to $\mathrm{C}_{12}$ spacers) were found to be crystalline but notoriously insoluble. ${ }^{20}$ These copolymers, with $\mathrm{C}_{7}$ to $\mathrm{C}_{12}$ spacer lengths showed a crystal to smectic transition upon prolonged annealing at $300^{\circ} \mathrm{C}$.

Attachment of longer chains to perylenebisimide impedes liquid crystalline ordering or nanowire formation. In order to explore the effect of nonaqueous media on the rod-coil systems for such morphologies, we previously studied ${ }^{21}$ a rod-coil system consisting of a flexible polymer and a rigid, large aromatic chromophore viz., perylenediimide. We prepared polydimethylsiloxane-co-perylenediimide (PDMS-coPTCDI), which could be considered a pseudo di-block copolymer, in which the PTCDI forms the short segment. This can also be considered a macromolecular surfactant applicable to non-aqueous systems. Similar to the case of amphiphilic block copolymer systems, micellar or vesicular morphologies were observed, depending on the blockspecific solvent used. A similar pseudo tri-block copolymer, PDMS-co-PTCDI-coPDMS formed nanoweb morphology. ${ }^{22}$ This was also the first case of a solvent-selective block copolymer in which one of the segments is a planar aromatic chromophore. 
Attaching a polymer to perylenediimide also facilitates the assembly of the photogenerator molecule in situ in a polymer matrix.

Whereas most of the reports on perylenediimides are based on organic solvents to meet solubility criteria, the study of self-assembly of polymer substituted perylenediimide in aqueous media is very attractive. In this paper, we discuss a rod-coil system, and an effective method to modulate the aggregate formation of the perylene derivative in water as well as in solvent mixtures, by competitive hydrophilic and hydrophobic interactions arising from attachments on the perylene diimide. We attached a hydrophilic polymer either to one side or both sides of a perylenediimide unit: Jeffamine-co-PTCDI or Jeffamine-co-PTCDI-co-Jeffamine, in which PTCDI is the short segment of the copolymer, with an $M_{w}$ one-fifth of Jeffamine. In the present case the Jeffamine attached to the imide nitrogen is water soluble where as the iso-butyl and the PTCDI groups are hydrophobic. The case of Jeffamine-co-PTCDI can also be considered an inverse surfactant since, contrary to the usual definition of a surfactant, the head group is hydrophobic and the tail is hydrophilic. We have prepared perylene diimides having hydrophobic short alkane chain on one side and hydrophilic Jeffamine ${ }^{\mathbb{R}}\left(\mathrm{M}_{\mathrm{W}}=2000\right)$ on the other side (MJ-PTCDI) and those having hydrophilic Jeffamine on both sides (DJPTCDI) (Scheme 3.1). 


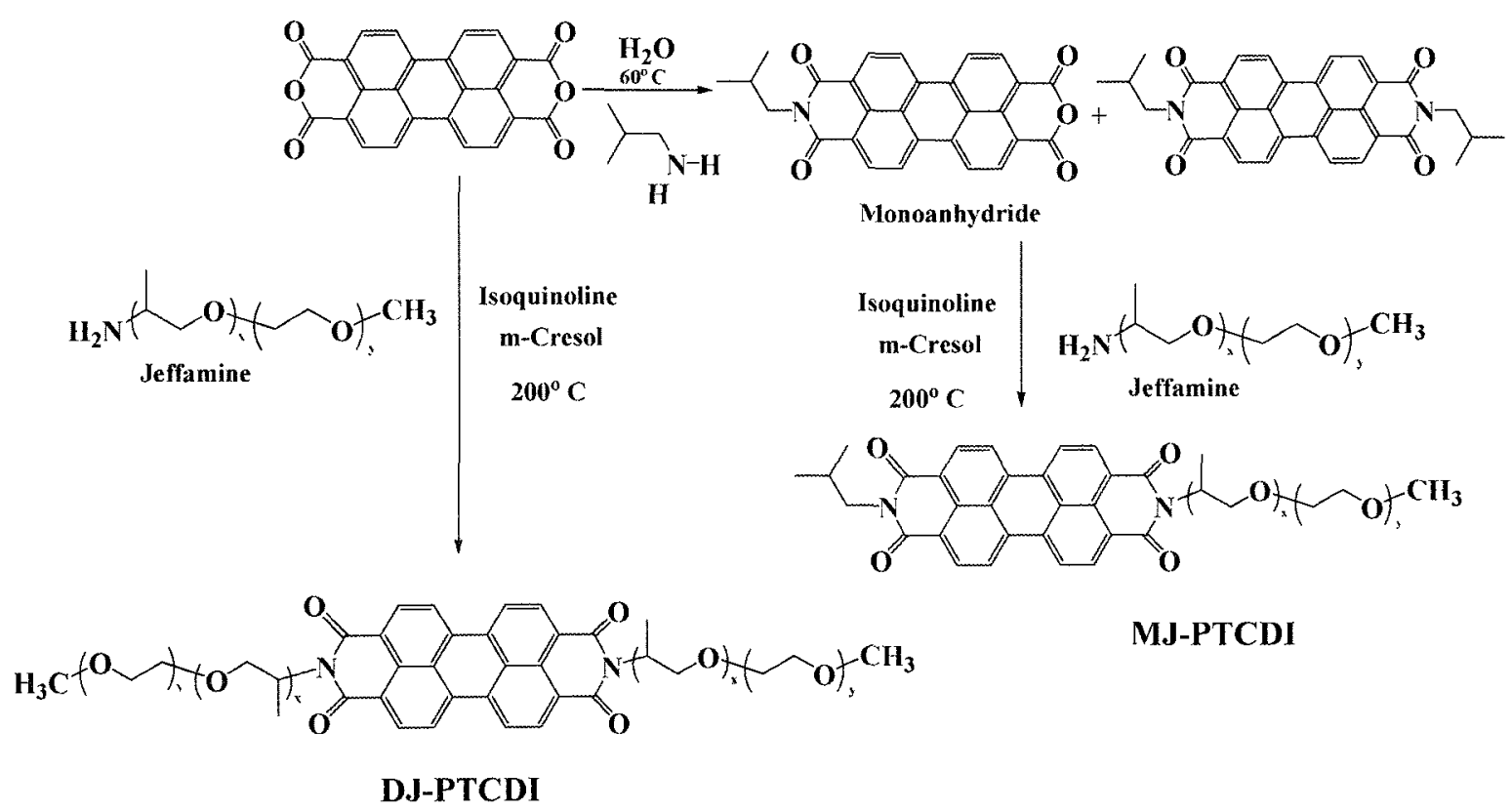

Scheme 3.1: Synthesis of DJ-PTCDI and MJ-PTCDI. ${ }^{23}$

\subsection{Experimental Section}

Perylene-3, 4, 9, 10-tertracarboxyl dianhydride, m-cresol, isoquinoline, and isobutyl amine were purchased from Aldrich Chemical Company and used as received. Mono functional, amine terminated ethylene oxide/propylene oxide copolymer (10/31 mole ratio) (Jeffamine ${ }^{(\mathbb{B})} \mathrm{M}-2070$ polyetheramine) of average molecular weight 2000 was purchased from Huntsman Corp. and used as received. Chloroform was purchased from Caledon Laboratories Ltd. and the other solvents were purchased from Aldrich Chemical Company Inc.

${ }^{1} \mathrm{H}$ NMR spectra were recorded on a Bruker-400 spectrometer using tetramethylsilane as an internal reference. Infrared measurements were performed either 
on a Michelson M120 BOMEM FTIR spectrometer or Varian 1000 Scimitar Series spectrometer in which $\mathrm{KBr}$ pellets were used for the powder samples of the synthesized monomer and $\mathrm{NaCl}$ discs were used for the waxy MJ-PTCDI and DJ- PTCDI. The waxy materials were dissolved in chloroform to make a dilute solution and then coated on $\mathrm{NaCl}$ discs. X-ray diffraction (XRD) data were collected using a Philips powder diffractometer (PW 1710) and nickel filtered $\mathrm{Cu} \mathrm{K}$ a radiation $(\lambda=1.542 \AA)$. The MDI Datascan 3.2 software (Materials Data Inc., Livermore, CA) was used for data collection. The results were analyzed with MDI Jade 5.0 XRD pattern processing software.

A Zeiss Axioplan 2 imaging universal optical microscope with Q-imaging Retiga digital camera was used to record optical micrographs in polarized modes, with Northern Eclipse (version 6.0) image processing software. The as-prepared samples were spread on glass slides. Samples from solutions were prepared by drop casting, as well as by freeze drying (The freeze drying was done in FREE ZONE ${ }^{\circledR} 4.5$ Liter Freeze Dry system by Labconco Corporation). A Tescan Vega-II XMU VP SEM was also used. The surface of the sample prepared for SEM was coated with gold $(80 \%)$ and palladium $(20 \%)$ alloy layer by HUMMER VII Sputtering System (ANATECH Ltd, Alexandria, VA.). The thickness of the conductive layer was 9-10 nm.

Thermal analysis was performed using a TA Instruments 2010 differential scanning calorimeter at a heating rate of $10^{\circ} \mathrm{C} / \mathrm{min}$. The samples were cooled to room temperature at a cooling rate of $5^{\circ} \mathrm{C} / \mathrm{min}$ or $2^{\circ} \mathrm{C} / \mathrm{min}$ in the second run. The instrument 
was calibrated for temperature and energy with indium and tin reference samples. DSC traces were recorded with about 7-10 $\mathrm{mg}$ of sample in an aluminum pan.

The UV-Vis spectroscopic measurements were performed at ambient conditions using a Varian CARY 3 UV-Vis spectrophotometer. The path length of the quartz sample cube was $10 \mathrm{~mm}$. Temperature dependent UV-Vis spectra were recorded with a CARY 300 Bio UV-Vis spectrophotometer with a Varian temperature controller (operating temperature $-10^{\circ} \mathrm{C}$ to $100^{\circ} \mathrm{C}$ ). The data were processed by CARY WinUV Software version 3.00. Fluorescent Spectra were recorded with a Varian CARY Fluorescent spectrophotometer at $\hat{\mathrm{c}}_{\mathrm{cx}} 460 \mathrm{~nm}$ and $417 \mathrm{~nm}$ with a bandwidth of $5 \mathrm{~nm}$ for excitation and $5 \mathrm{~nm}$ for emission. Data collection and processing were done by Eclipse WinFLR Software (version 1.1).

The Dynamic Light Scattering (DLS) experiment was done with a NanoDLS particle size analyzer (Brookhaven Instruments Corp) at $25^{\circ} \mathrm{C}$. The wavelength of the laser was set at $637 \mathrm{~nm}$. The machine was calibrated with standard polystyrene nanoparticle of $90 \mathrm{~nm}$ diameter. The effective diameter is an average of 10 runs each time. The solutions were made in water at $10^{-3} \mathrm{M}$ concentration. Effective diameter was calculated using the NanoDLS Particle Sizing Software Ver. 4.11.

\subsubsection{Synthesis of $\mathrm{N}$-isobutyl-3, 4, 9, 10-perylenetetracarboxylic}

\section{Monoanhydride Monoimide.}

The title compound was prepared by the procedure published by Y. Nagao et al

(Scheme 3.1$).{ }^{23}$ In a round-bottom flask, $7.6 \mathrm{mmol}$ of perylene-3,4.9,10-tetracarboxylic 
dianhydride was dispersed in water and slowly heated to $60^{\circ} \mathrm{C}$. Excess isobutyl amine (61.2 mmol), dissolved in water, was added to the reaction mixture at the same temperature and stirred vigorously for $4 \mathrm{hrs}$. The reaction mixture was then acidified with $10 \% \mathrm{HCl}$ and the precipitate formed was filtered and washed with water to remove excess amine. The collected precipitate was dissolved in $1 \%$ hot potassium hydroxide. The target material, which was soluble in hot $\mathrm{KOH}$, was separated from the insoluble diimides by vacuum filtration. To the filtrate $\mathrm{KCl}$ was added until $10 \%$ concentration and the precipitated potassium salt of perylene monoimide monoanhydride was filtered. The solid was dissolved in water and acidified with $10 \% \mathrm{HCl}$ and the precipitate formed was filtered, washed with water and dried to yield the desired product (yield $80 \%$ ). The FT-IR spectrum reveals the presence of both anhydride $\left(1700-1740 \mathrm{~cm}^{-1}\right)$ and imide carbonyl (1697 and $1654 \mathrm{~cm}^{-1}$ ) absorption frequencies confirming the formation of the compound (Appendix A, Figure A1). ${ }^{1}$ HNMR spectra also confirms the formation of perylene monoanhydride.

\subsubsection{Synthesis of MJ-PTCDI}

In a round-bottom flask, $0.1 \mathrm{mmol}$ of perylene monoimide monoanhydride, 0.1 mmol of Jeffamine, and $0.2 \mathrm{~g}$ of isoquinoline were dispersed in $15 \mathrm{~mL}$ of $m$-cresol, and the solution was slowly heated to $200{ }^{\circ} \mathrm{C}$ (Scheme 3.1). The reaction mixture was stirred at $200{ }^{\circ} \mathrm{C}$ for $4 \mathrm{~h}$, cooled to room temperature and poured into chloroform. The solution was filtered to remove the unreacted perylene monoimide mono anhydride, if any. The filtrate was washed with acidified water in a separating funnel to remove the unreacted Jeffamine, if any, and the organic layer was separated and dried to yield red waxy 
material (yield 78\%). FTIR (KBr, Chloroform): 2870 (-C-H stretching), 1702 (imide), 1653 (imide), 1597 (C-C stretching), 1456 (C-H bending), 1357 (C-H rocking). 1103 (CO bending), 948 (Ar-CH out of plane bending), 864 (Ar-CH out of plane bending), 745.2 (C-H rocking) $\mathrm{cm}^{-1}$ (Appendix A. Figure A3). In the FT-NMR spectroscopy, the resonance from the perylene moiety in aromatic region (8.8-8.6 ppm multiplet) and the ethylene oxide moiety in aliphatic region also confirmed the completion of reaction (Appendix B, Figure B2).

\subsubsection{Synthesis of DJ-PTCDI}

In a round-bottom flask, $0.1 \mathrm{mmol}$ of 3,4,9,10-perylenetetracarboxylic dianhydride, $0.2 \mathrm{mmol}$ of Jeffamine ${ }^{\sqrt{k}}$, and $0.2 \mathrm{~g}$ of isoquinoline were dispersed in $15 \mathrm{~mL}$ of m-cresol, and the solution was slowly heated to $200{ }^{\circ} \mathrm{C}$ (Scheme 3.1). The reaction mixture was stirred at $200{ }^{\circ} \mathrm{C}$ for $4 \mathrm{~h}$, cooled to room temperature and poured into chloroform. The solution was filtered to remove the unreacted $3,4,9,10$ perylenetetracarboxylic dianhydride if any. The filtrate was collected and it was washed with acidified water in a separating funnel to remove the unreacted Jeffamine, if any and the organic layer was separated and dried to yield a red waxy material (yield 85\%). FTIR (KBr, Chloroform): 2858 (-C-H stretching), 1698 (imide), 1656 (imide), 1594 (C-C stretching), $1457(\mathrm{C}-\mathrm{H}$ bending $) .1094(\mathrm{C}=\mathrm{O}$ stretching $), 850.1(\mathrm{Ar}-\mathrm{CH}$ out of plane bending), 747.3 (C-H rocking) $\mathrm{cm}^{-1}$ (Appendix A, figure A4). In the FT-NMR spectra, the resonance from the perylene moiety in the aromatic region (8.8-8.6 ppm. multiplet) and the ethylene oxide moiety in aliphatic region also confirmed the completion of reaction (Appendix B, figure B3). 


\subsection{Results and Discussion:}

\subsubsection{UV-Vis and Fluorescence Spectroscopy:}

Since the rod segment is a chromophore, absorption spectroscopy is a convenient technique to study the association in solution. Figure 3.1 shows the UV absorption spectra of both compounds at various concentrations in water. At low concentrations, when there is no aggregation, the typical perylene monomeric absorption pattern is seen with the intensities of the peaks $\mathrm{I}_{468}<\mathrm{I}_{495}<\mathrm{I}_{530} \mathrm{~nm}$ for DJ-PTCDI and at $\mathrm{I}_{472}<\mathrm{I}_{498}>\mathrm{I}_{547}$ $\mathrm{nm}$ for MJ-PTCDI. Upon increasing the concentration, the $495 \mathrm{~nm}$ peak shows higher intensity with DJ-PTCDI, and in the case of MJ-PTCDI, the intensity of the $472 \mathrm{~nm}$ peak increases relative to that of $498 \mathrm{~nm}$. Such intensity reversal is related to the formation of aggregates. $^{15 \mathrm{a}, 19 \mathrm{c}}$ Although both MJ-PTCDI and DJPTCDI formed aggregates in water, MJPTCDI exhibited stacking at a higher concentration $\left(10^{-5} \mathrm{M}\right)$ compared to the latter $\left(10^{-7} \mathrm{M}\right)$. The tendency in both cases would be to maximize the contact of the hydrophilic polyether chain with water. The ratios of molecular weights of hydrophilic and hydrophobic parts in MJ-PTCDI and DJ-PTCDI are 4.63 and 11.09 , respectively. The lower critical solution temperature (LCST) of Jeffamine is $30^{\circ} \mathrm{C}$. In the case of DJPTCDI, the hydrophilic Jeffamine ${ }^{\mathbb{B}}$ on both ends can be expected to be fully extended at room temperature due to hydrophilicity, which would promote close-stacking and aggregation of perylene cores at a lower concentration. This shows that the aggregation can be controlled by hydrophilic/ hydrophobic ratio. Figure 3.1 shows that with DJPTCDI the intensity reversal occurred between the peaks at 495 and $530 \mathrm{~nm}$ corresponding to $\mathrm{S}_{0-1}$ to $\mathrm{S}_{0-0}$ transitions, whereas in MJ-PTCDI the intensity reversal occurred between 472 and $498 \mathrm{~nm}$ peaks corresponding to $S_{0-2}$ to $S_{0-1}$ transitions. 

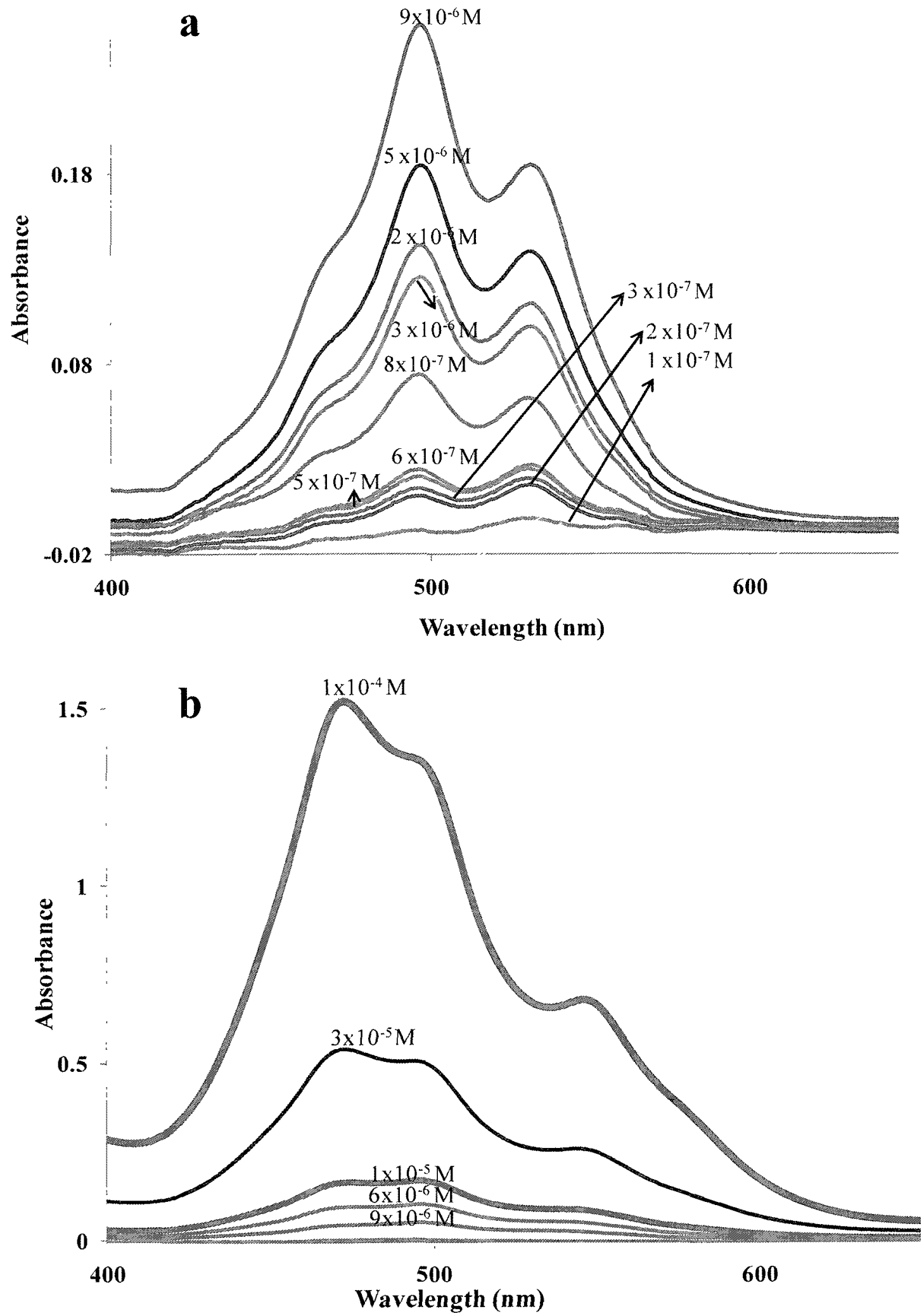

Figure 3.1: UV absorption spectra of DJ-PTCDI (a) and MJ-PTCDI (b) at increasing concentrations at the direction of the arrow from $1 \times 10^{-7}$ to $9 \times 10^{-5} \mathrm{M}$. 
Typically, the absorption maxima are blue shifted with an increase in concentration in the case of H-type aggregates, and the fluorescence is quenched. In contrast, in J-type aggregates the absorption maxima are red shifted and there is no fluorescence quenching. ${ }^{19 a, 9,24-26}$ However in the present case the absorption spectra did not indicate the type of packing since there was no shift in the absorption maxima with increase in concentration. The aggregation does not give rise to any new peak ${ }^{15 a}$ as reported for short chain compounds, rather quenching of fluorescence intensity beyond a certain aggregation range was observed. The emission spectra of the DJ-PTCDI and MJPTCDI exhibited different behavior (figures 3.2 and 3.3 respectively). Generally the reduction in intensity of the longer wavelength peak is correlated to the H-type aggregation. In the UV-Vis absorption spectra of DJ-PTCDI the aggregation occurred at a very low concentration, i.e. $10^{-7} \mathrm{M}$ but the decrease in fluorescence was observed only at $10^{-5} \mathrm{M}$. The absence of quenching in fluorescence intensity with the aggregation might be due to the formation of J-type aggregates and the observed quenching at higher concentration might be due to photo-induced electron transfer within the aggregates of higher order. ${ }^{24}$ MJ-PTCDI showed quenching in the fluorescence intensity with the beginning of aggregation observed in UV-Vis absorption spectra, possibly due to the formation of H-type aggregates. In DJ-PTCDI the attachment of long polymer chain on both sides of PTCDI prevented the H-type packing leading to J-type aggregates, where as in MJ-PTCDI, due to the chain attachment on one side the perylene formed H-type packing. In general, the fluorescence intensity of MJ-PTCDI was several folds lower than the DJ-PTCDI, which can be attributed to strong $\pi-\pi$ stacking. 

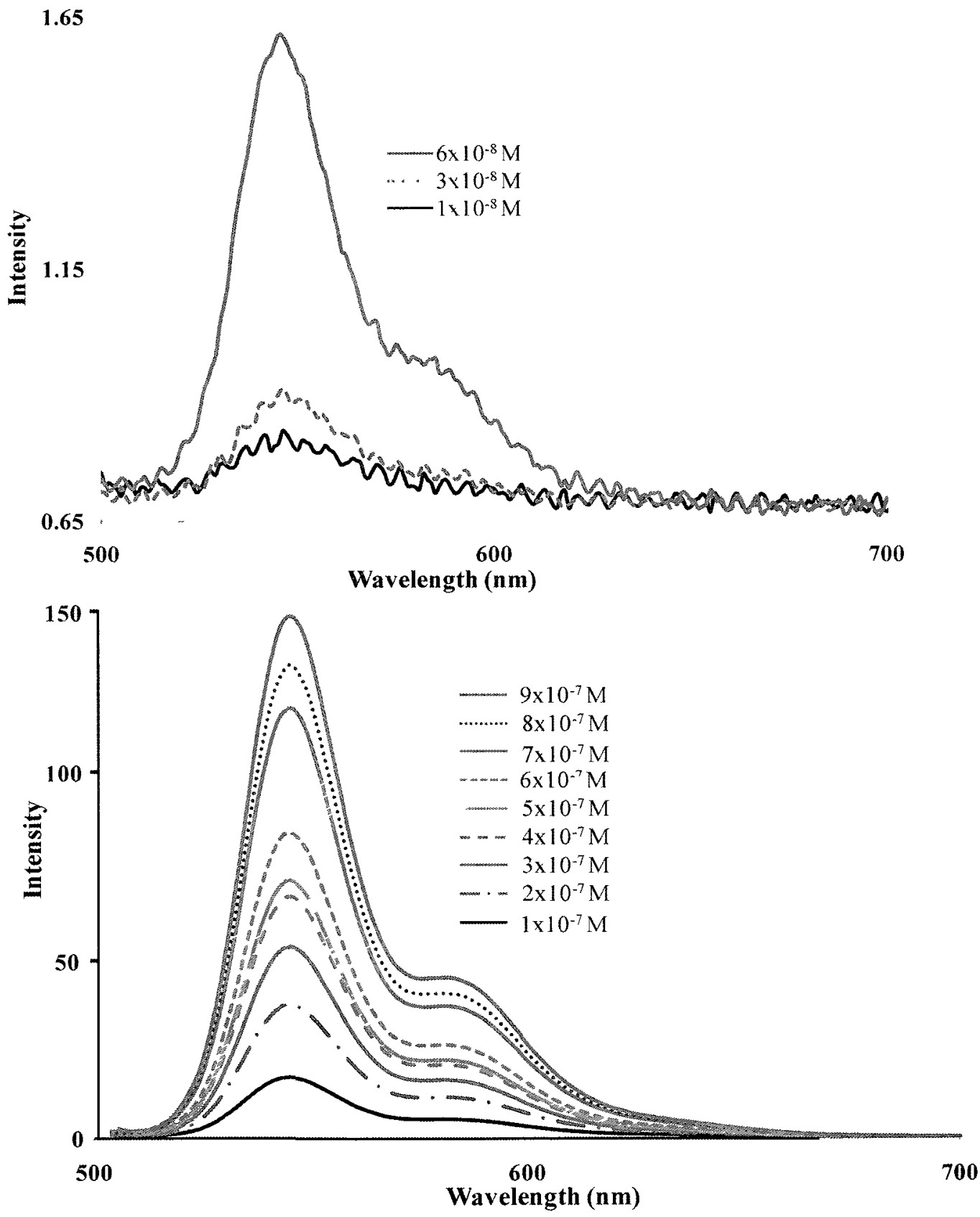

Figures continued in the next page 

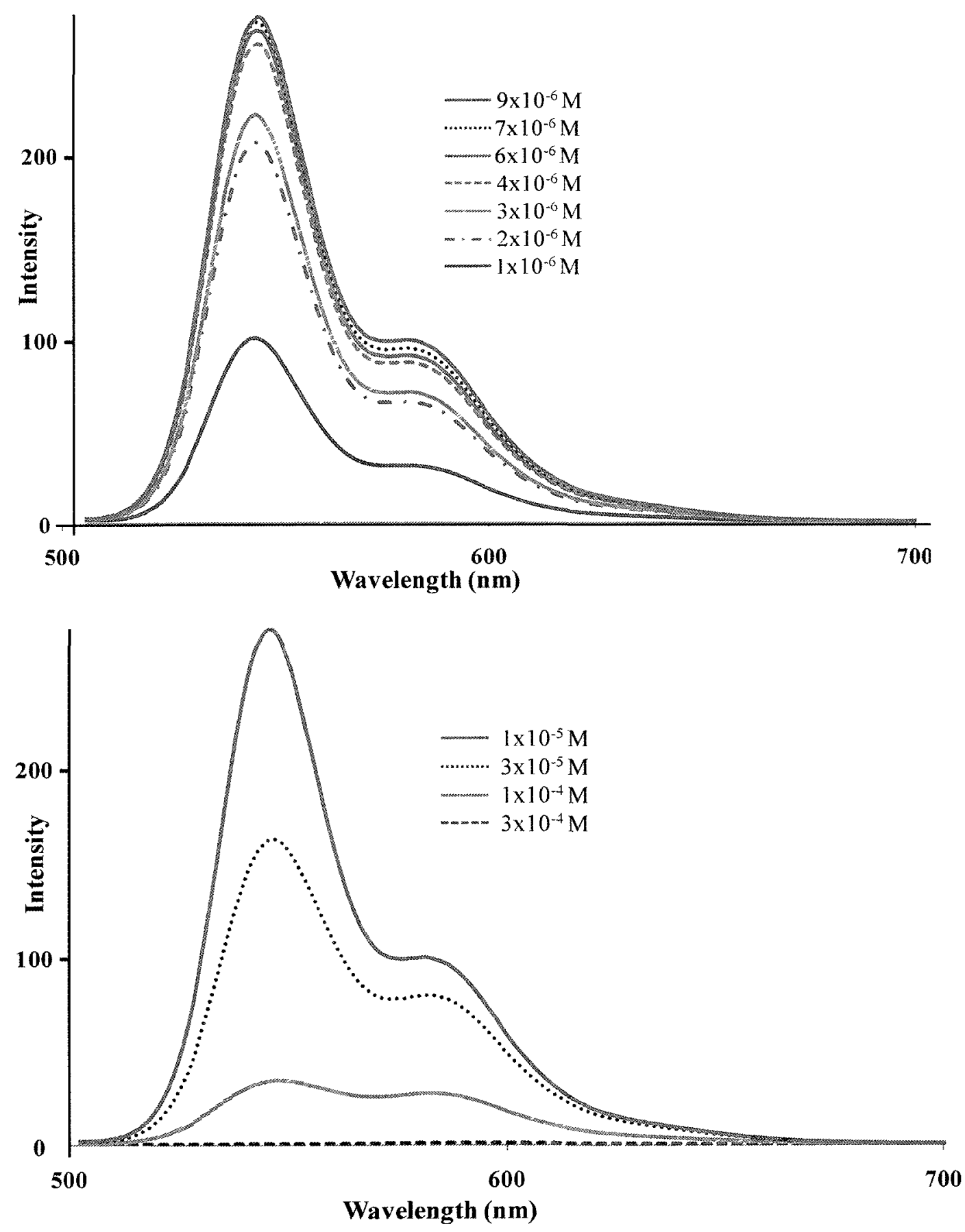

Figure 3.2: Concentration dependent fluorescence spectra of DJ-PTCDI in water. 

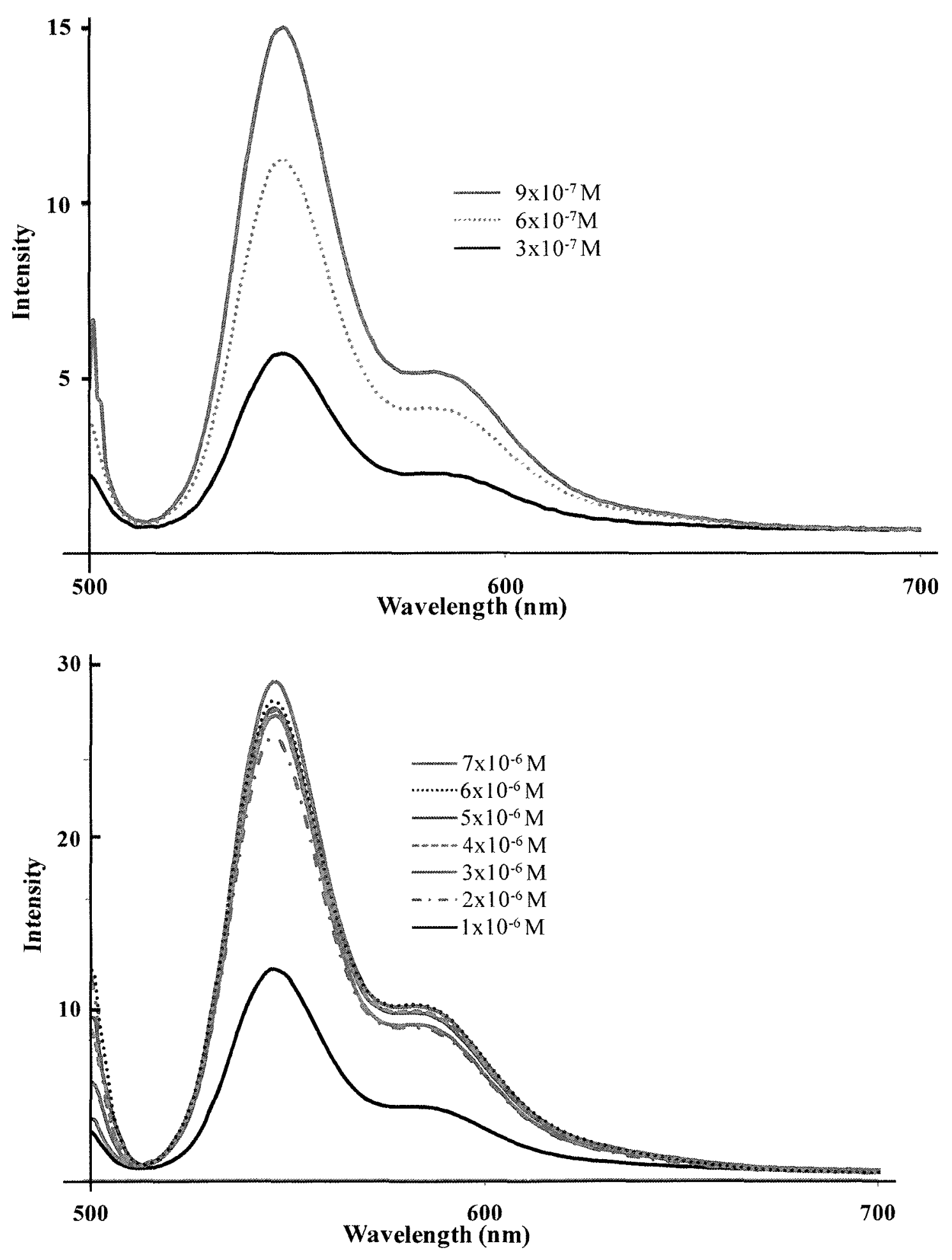

Figures continued in the next page 

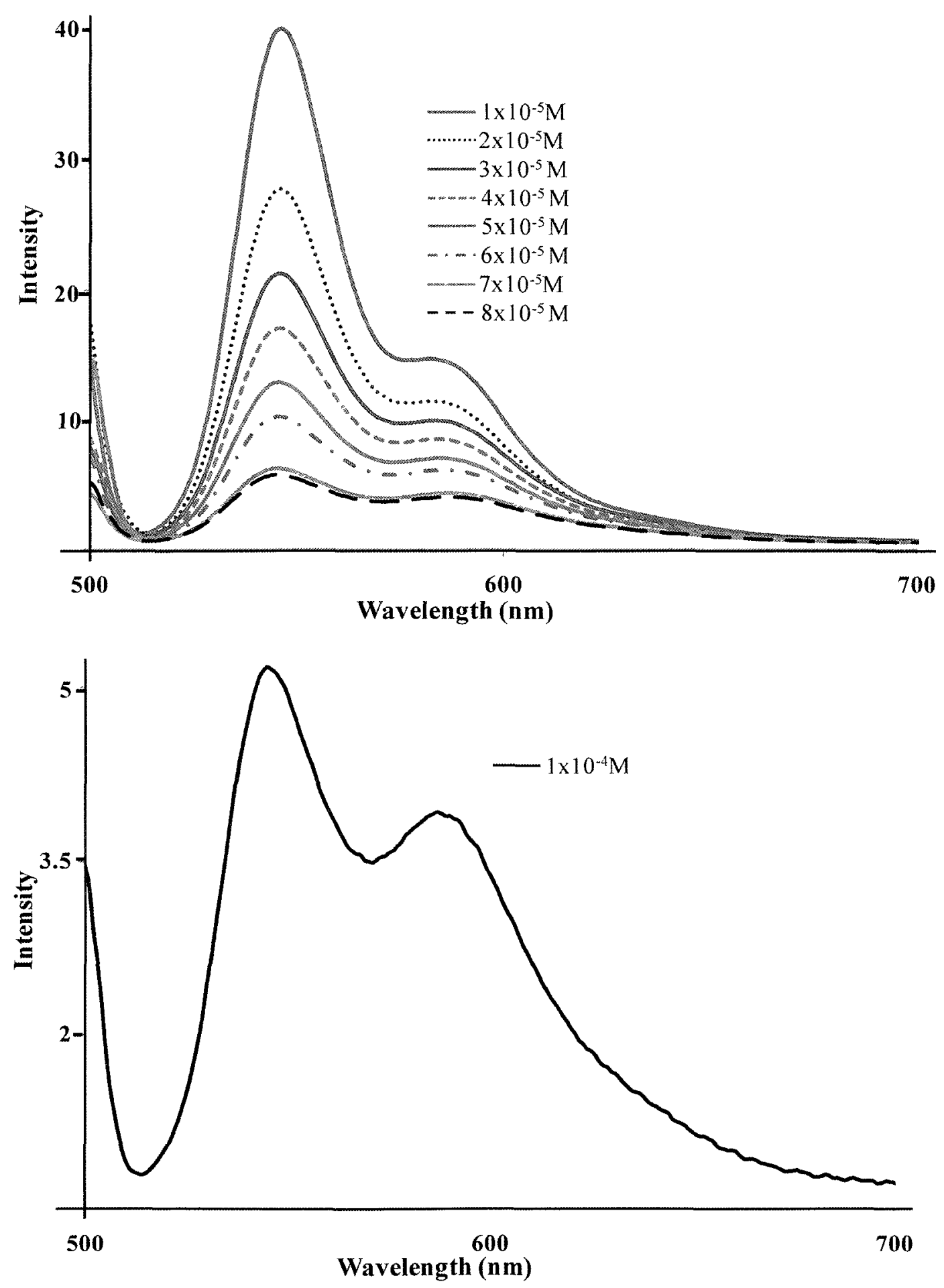

Figure 3.3: Concentration dependent fluorescence spectra of MJ-PTCDI in water. 


\subsubsection{Effect of Solvent}

Previous reports on perylene molecules with short-chain substituents indicate that stacking occurs with the addition of a non-solvent ${ }^{15 a}$ that cannot dissolve the $\pi$ aggregates (the effect of solubility of the hydrophobic short side chains is insignificant). But with the long polymer chains $\left(M_{u}=2000\right)$ compared to hydrophobic short chain, the side chain interaction with the solvents is expected to affect the aggregation. No stacking was observed with both MJ-PTCDI and DJ-PTCDI in solvents such as acetone and chlorinated hydrocarbons that can solvate both the $\pi$ aggregates and the side chain. Figure 3.4 for both MJ-PTCDI and DJ-PTCDI in acetone, $\mathrm{CH}_{2} \mathrm{Cl}_{2}$, chloroform, DMAc, DMF, methanol, and THF shows identical spectra (monomeric absorption pattern), with peaks at 453,482 , and $519 \mathrm{~nm}$ with the latter being most intense ( Excitation of these solutions at $366 \mathrm{~nm}$ gives intense fluorescence color which are shown in Appendix C, figure C1 and $\mathrm{C} 2$ ). 


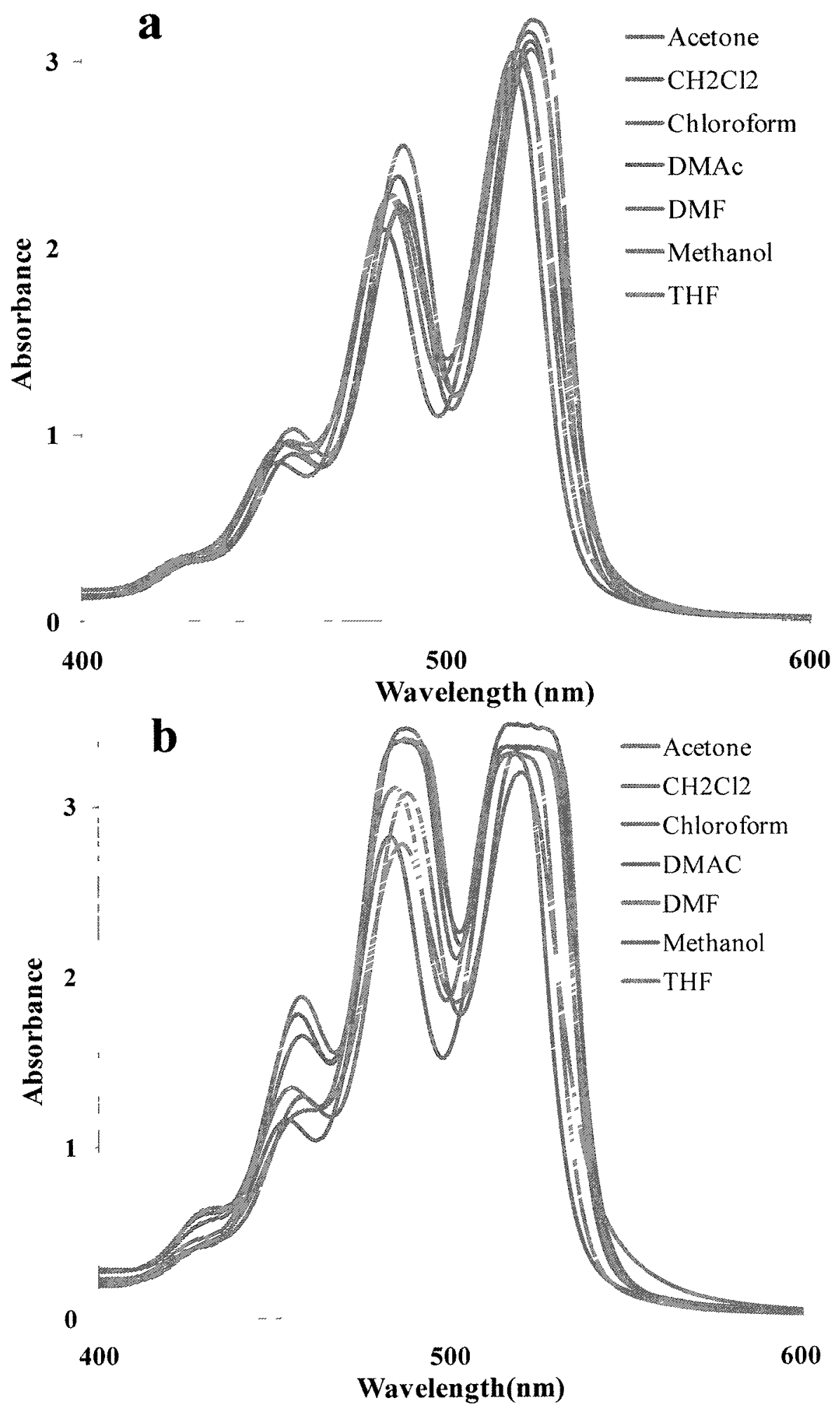

Figures continued in the next page 


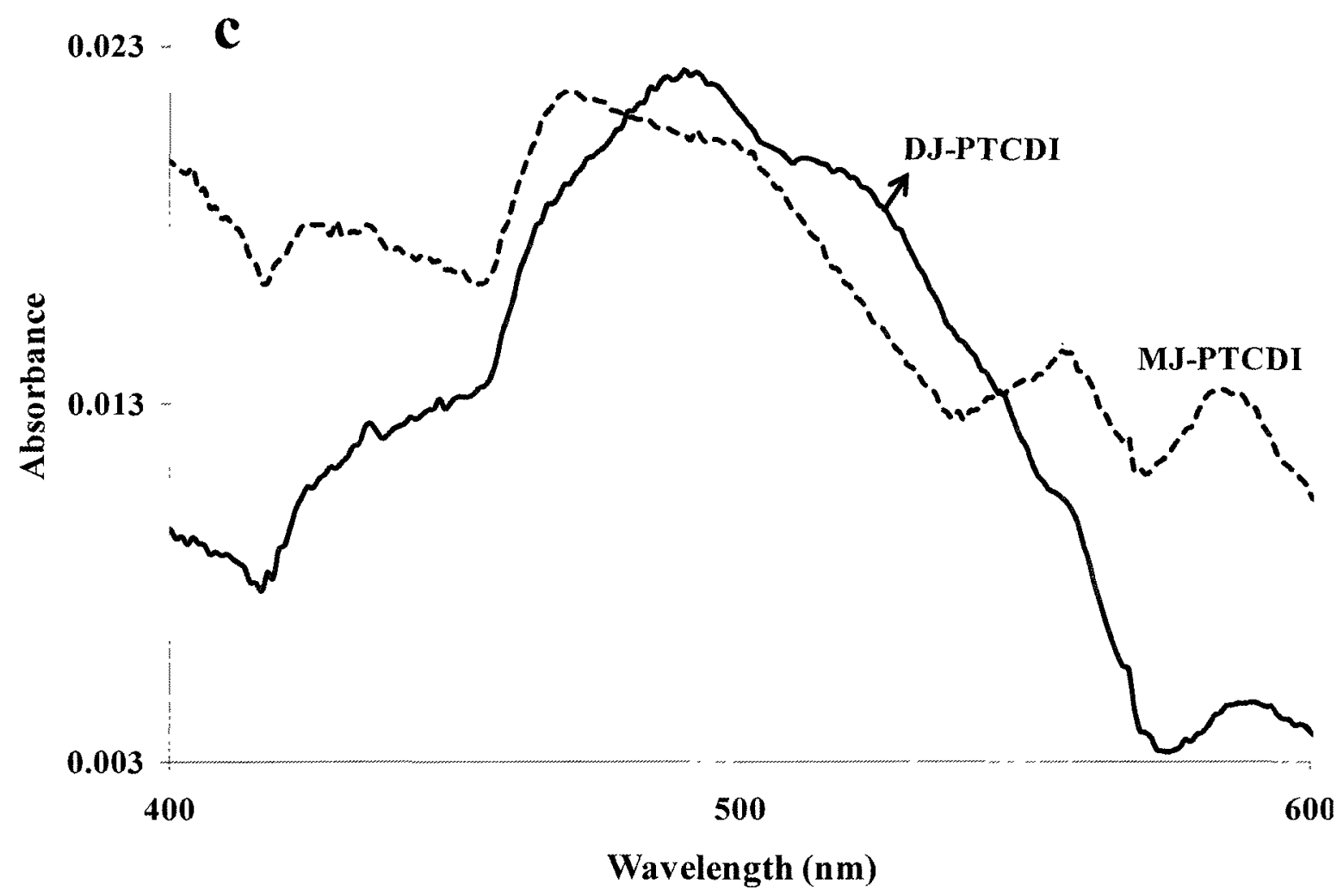

Figure 3.4: Absorption spectra of (a) DJPTCDI and (b) MJPTCDI in different solvents at $10^{-4} \mathrm{M}$; (c) in solid state from drop-cast films from $10^{-4} \mathrm{M}$ aqueous solution.

Figure 3.5 shows that when hexane, a non-solvent to both the rigid perylene and the side chain was added to the $\mathrm{CH}_{2} \mathrm{Cl}_{2}$ solution, no aggregation was observed even at high solvent/non solvent ratio. This might be due to the collapsed globular state of the polymer chain in the non-solvent which inhibited stacking. If the solvent (e.g., water) can solubilize the polymer chain and act as a non-solvent to the $\pi$ aggregates, it leads to selfassembly as observed earlier. In the absorption spectra of both compounds, typical intensity reversal was observed (figure 3.6) when water was added to the solution in 
acetone indicating the formation of aggregates. This shows that in case of perylene having long chain molecules the solvent interaction can be effectively used to control the self-assembly of perylene chromophores. As seen in figure 3.6, with the change in acetone/water ratio both the compounds exhibited gradual change to aggregated species indicating the dynamic formation of aggregates. ${ }^{27}$ The spectral response also becomes broader without vibronic fine structures with an increase in aggregation. ${ }^{28}$ Apart from intensity reversal, absorption maxima of both compounds exhibited a red shift with an increase in water content, indicating J-type aggregation. 

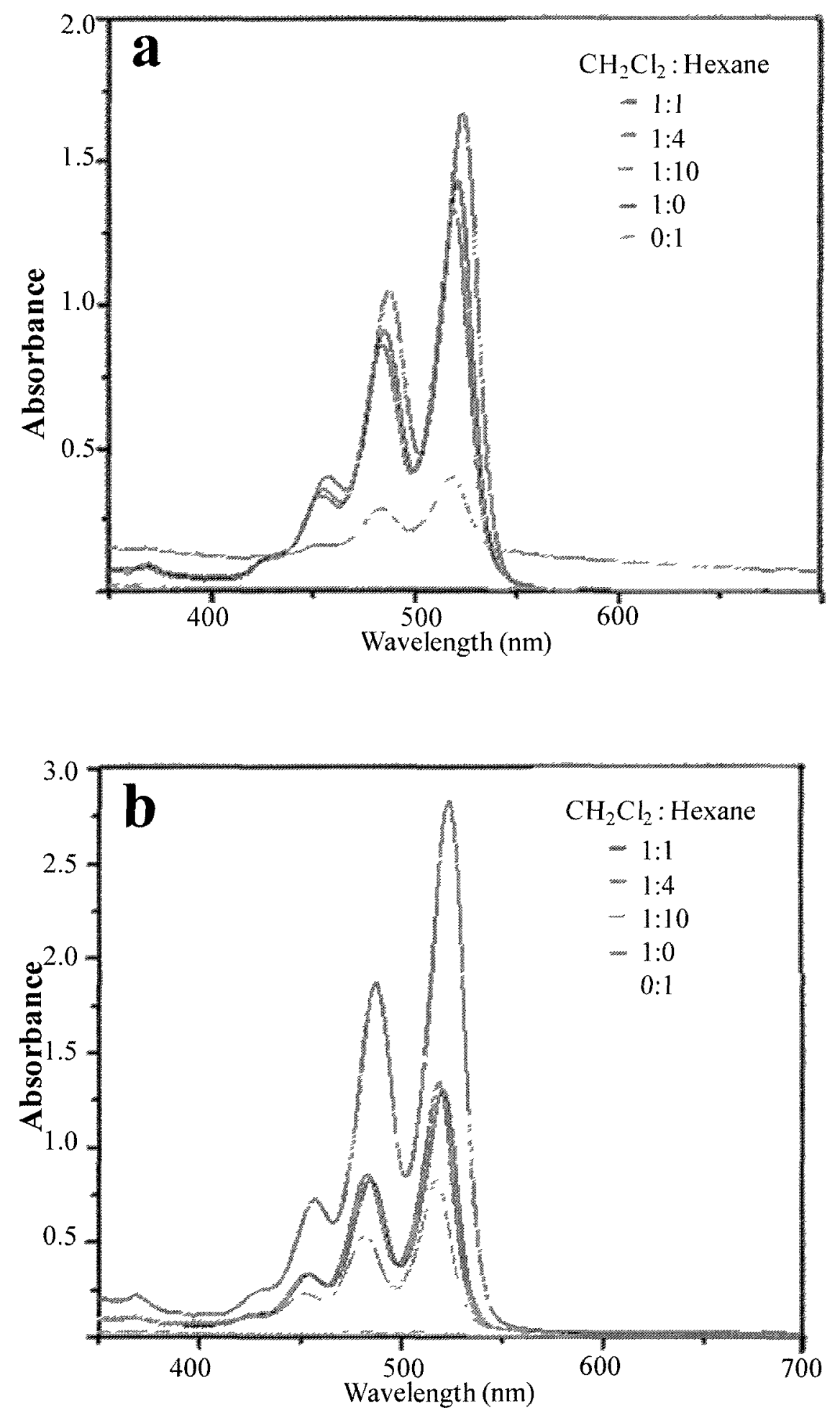

Figure 3.5: UV Visible spectra of DJ-PTCDI (a) and MJ-PTCDI in $\mathrm{CH}_{2} \mathrm{Cl}_{2}$ : Hexane solvent mixtures at $10^{-5} \mathrm{M}$. 


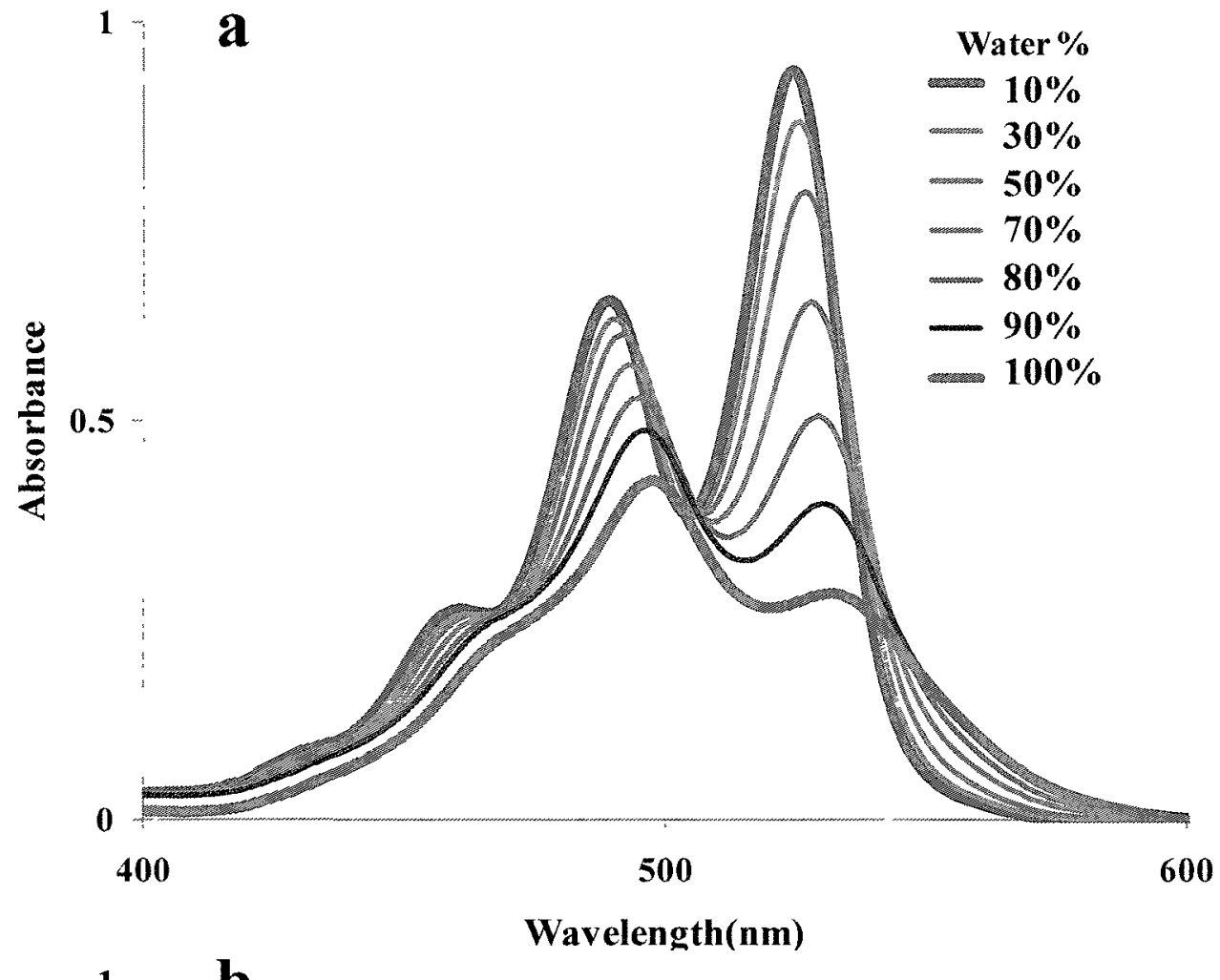

$1 \div \mathbf{b}$

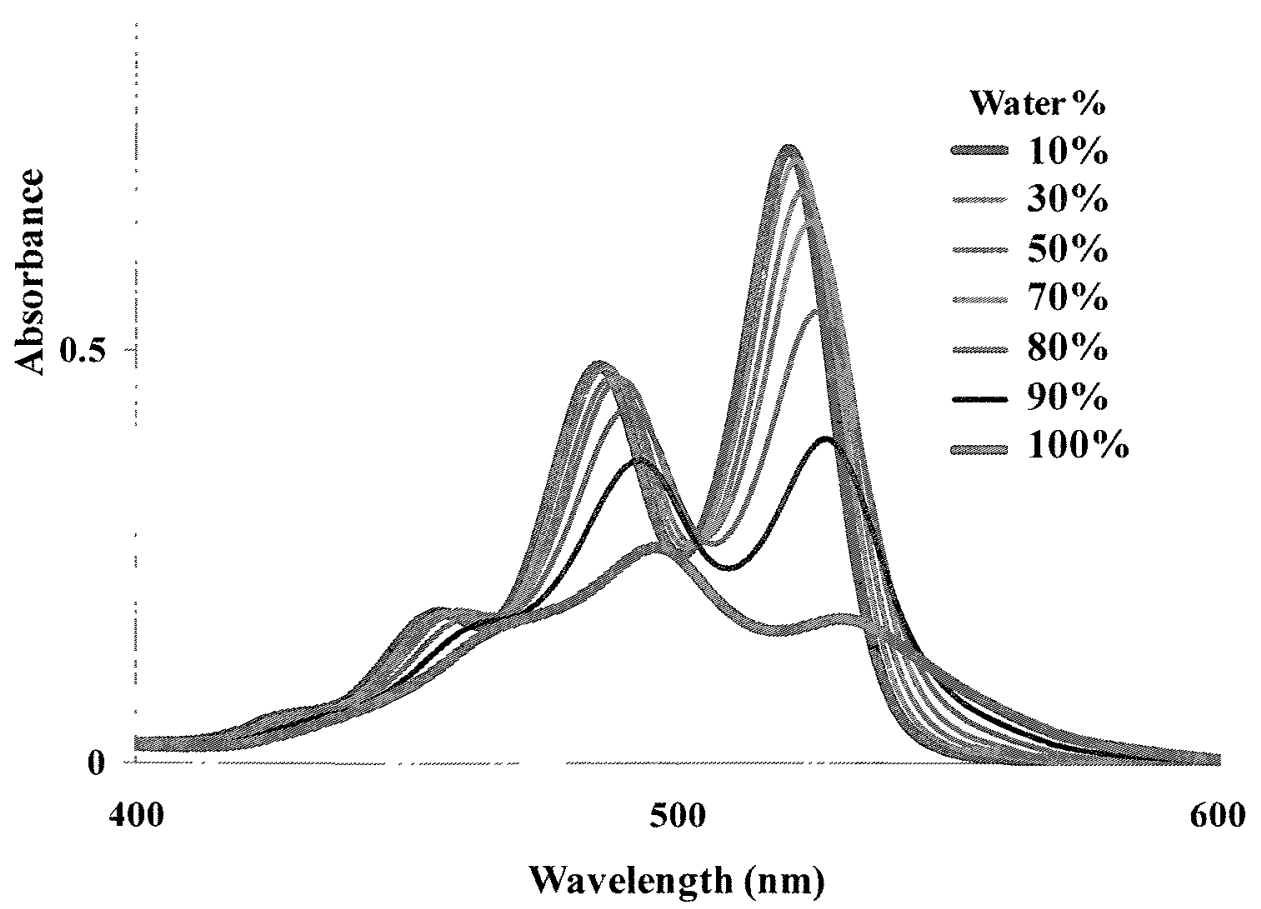

Figure 3.6: UV-Vis absorption spectra of DJ-PTCDI (a) and MJ-PTCDI (b) in Acetone/Water mixture at $10^{-5} \mathrm{M}$. 

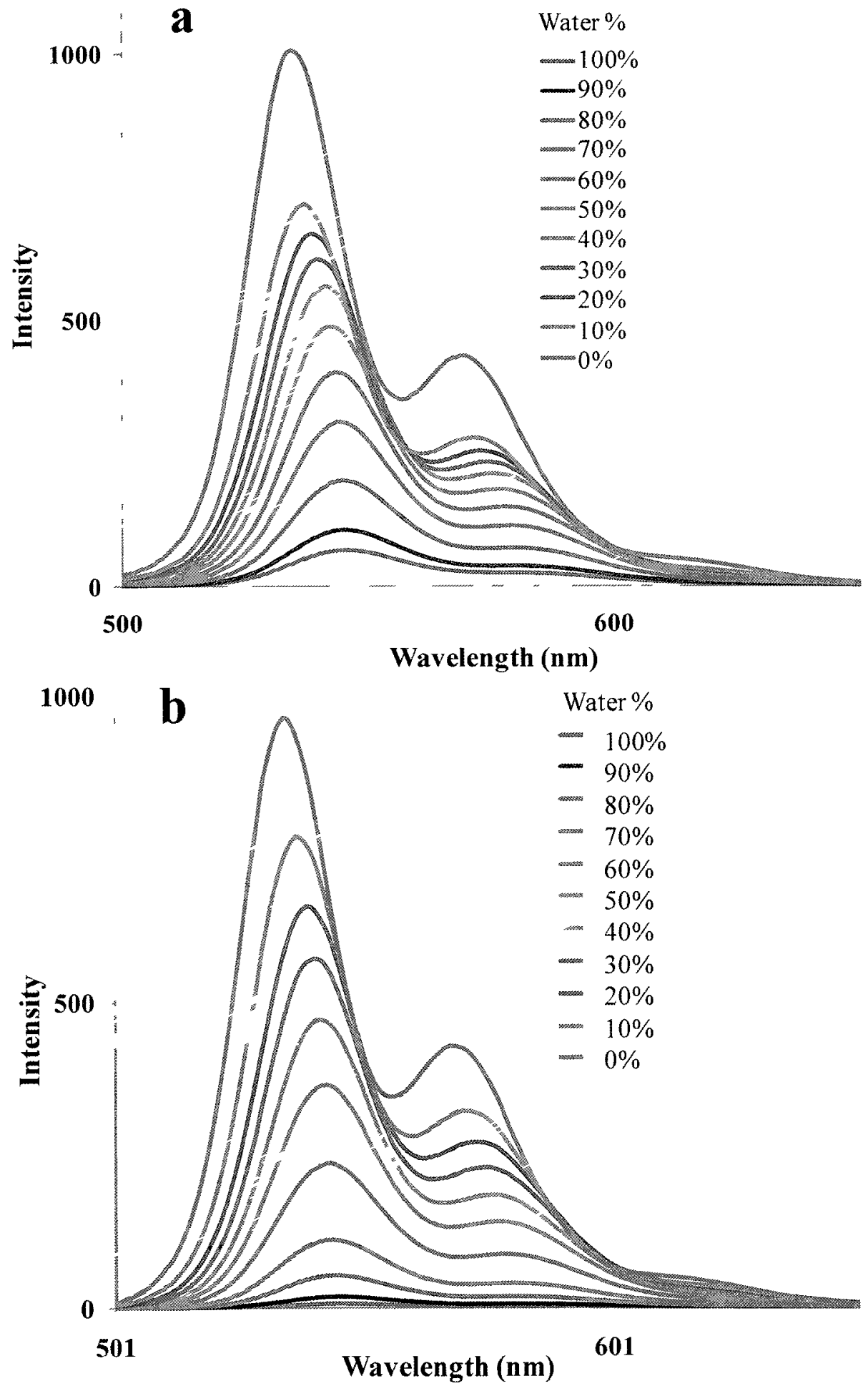

Figure 3.7: Fluorescence emission spectra of DJ-PTCDI (a) and MJ-PTCDI (b) in acetonewater binary mixtures at $10^{-5} \mathrm{M}$. 
However, the emission spectra (figure 3.7) in the binary solvents displayed fluorescence quenching despite the formation of J-type aggregates. This may be due to the formation of higher order aggregates. ${ }^{29}$ The emission color arising from excitation at $366 \mathrm{~nm}$ of the solutions of binary solvents with acetone: water 1:9 also shows diminishing intensity confirming the aggregate structure of DJPTCDI and MJPTCDI (Appendix C. figure $\mathrm{C} 3$ and $\mathrm{C} 4$ respectively). The fluorescent spectra of both DJ-PTCDI and MJ-PTCDI in figure 3.7 show a hypsochromic shift of $\lambda$ max with hyperchromic shift in intensity of $\lambda_{\max }$ with increasing acetone concentration.

The mole fraction of aggregate was estimated for both of the diimides in acetonewater mixture by using equation ${ }^{30} 3.1$.

$$
\alpha_{\text {agg }} \approx\left(\mathrm{A}_{\text {mi }}(\mathrm{T})-\mathrm{A}_{\text {nonagg }}\right) /\left(\mathrm{A}_{\text {agg }}-\mathrm{A}_{\text {nonagg }}\right)
$$

Here, $\alpha_{a g g}$ is the mole fraction of aggregate at temperature $T$, and $A_{\operatorname{mix}}(T)$ is the absorbance at $\lambda_{\max }$ of the monomer (538 $\mathrm{nm}$ for DJ-PTCDI and 520 for MJ-PTCDI) at a given mixture of solvent, $\mathrm{A}_{\text {nonagg }}$ and $\mathrm{A}_{\mathrm{agg}}$ are the absorbances of the non-aggregated state and of the maximum aggregate state, respectively.

Figure 3.8 shows that $\alpha_{\text {agg }}$ increases almost linearly with water content for DJ-PTCDI, whereas the increase appears to be faster beyond $40 \%$ water concentration in the case of MJ-PTCDI. Upon excitation at $366 \mathrm{~nm}$. DJ-PTCDI in mixed solvents shows no significant change in the intensity of the color of the fluorescent emission. Green 
emission with almost equal intensity was seen with increasing acetone concentration. However, with excitation at $366 \mathrm{~nm}$, MJ-PTCDI gave yellow fluorescence with increasing intensity with acetone concentration, and with $100 \%$ acetone, green emission was seen.

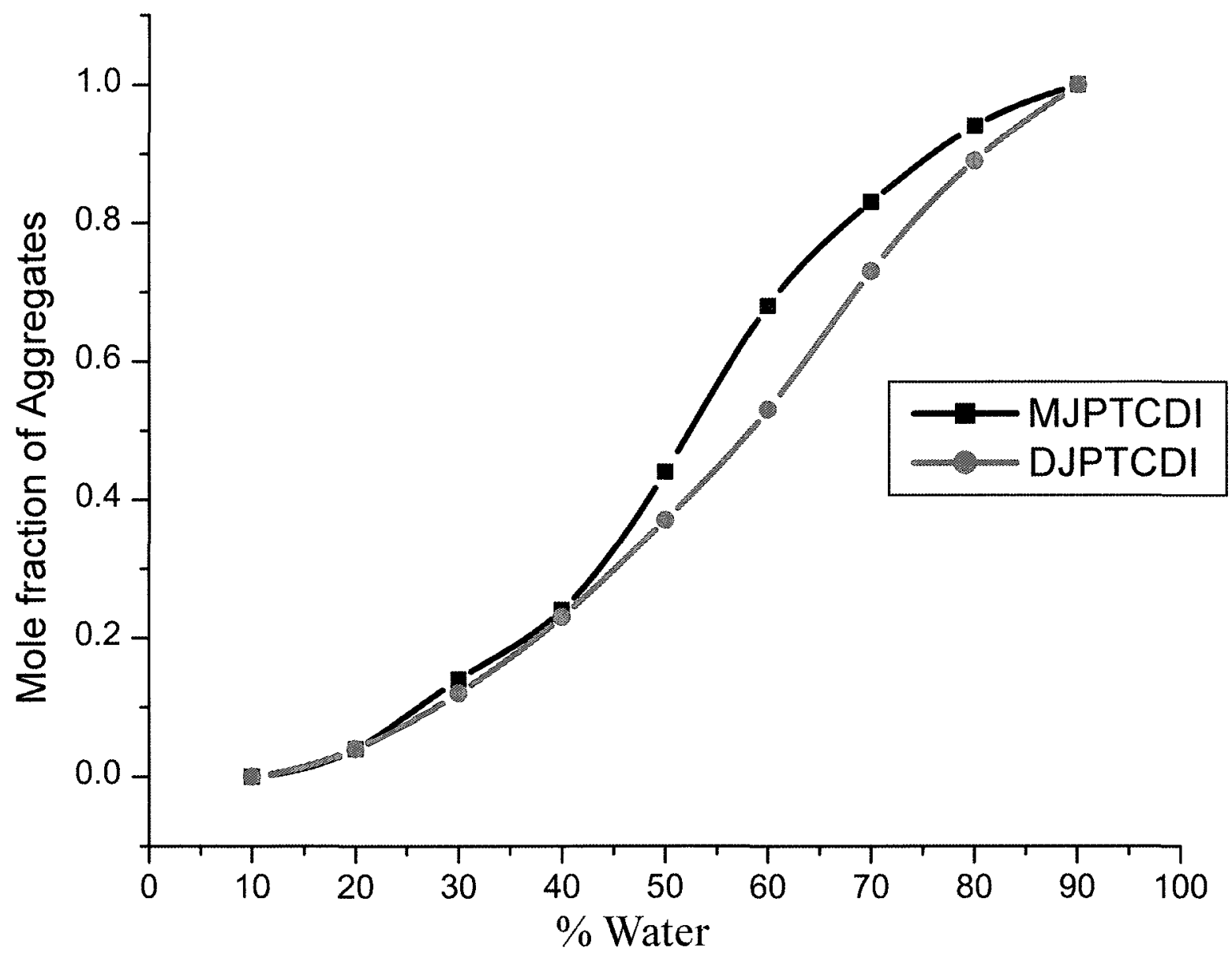

Figure 3.8: Mole fraction of aggregates $\left(\alpha_{\mathrm{agg}}\right)$ as a function of solvent composition (water/acetone) for MJ-PTCDI and DJ-PTCDI. 


\subsubsection{Aggregate Stability}

The stability of the $\pi$-mediated aggregation with $\mathrm{pH}$ and temperature was studied. Figure 3.9 shows the $\mathrm{pH}$ dependent absorption spectra of DJ-PTCDI and MJ-PTCDI solutions. With DJ-PTCDI no significant change is seen in the range of $\mathrm{pH}$ from 1 to 13 except the slight hyperchromic shift with increasing $\mathrm{pH}$, indicating that there is no effect on the aggregate. At $\mathrm{pH}=14$ intensity reversal between $\mathrm{S}_{0-1}$ and $\mathrm{S}_{0-2}$ vibronic peak is seen. Similarly, in the case of MJ-PTCDI there was no significant change in the absorption pattern up to $\mathrm{pH}=12$. At $\mathrm{pH}=13$ the vibronic transition for $\mathrm{S}_{0-0}$ and $\mathrm{S}_{0-1}$ becomes a broad diffused amalgamated peak spanning from $490-540 \mathrm{~nm}$ and at $\mathrm{pH}=14$ the absorption pattern becomes that of the monomeric form with a hypsochromic shift of $40 \mathrm{~nm}$, showing destabilization of the aggregates. The emission color arising from excitation at $366 \mathrm{~nm}$ of the solutions of DJPTCDI and MJPTCDI at different $\mathrm{pH}$ solution is given in Appendix C (figure C5 and C6). 

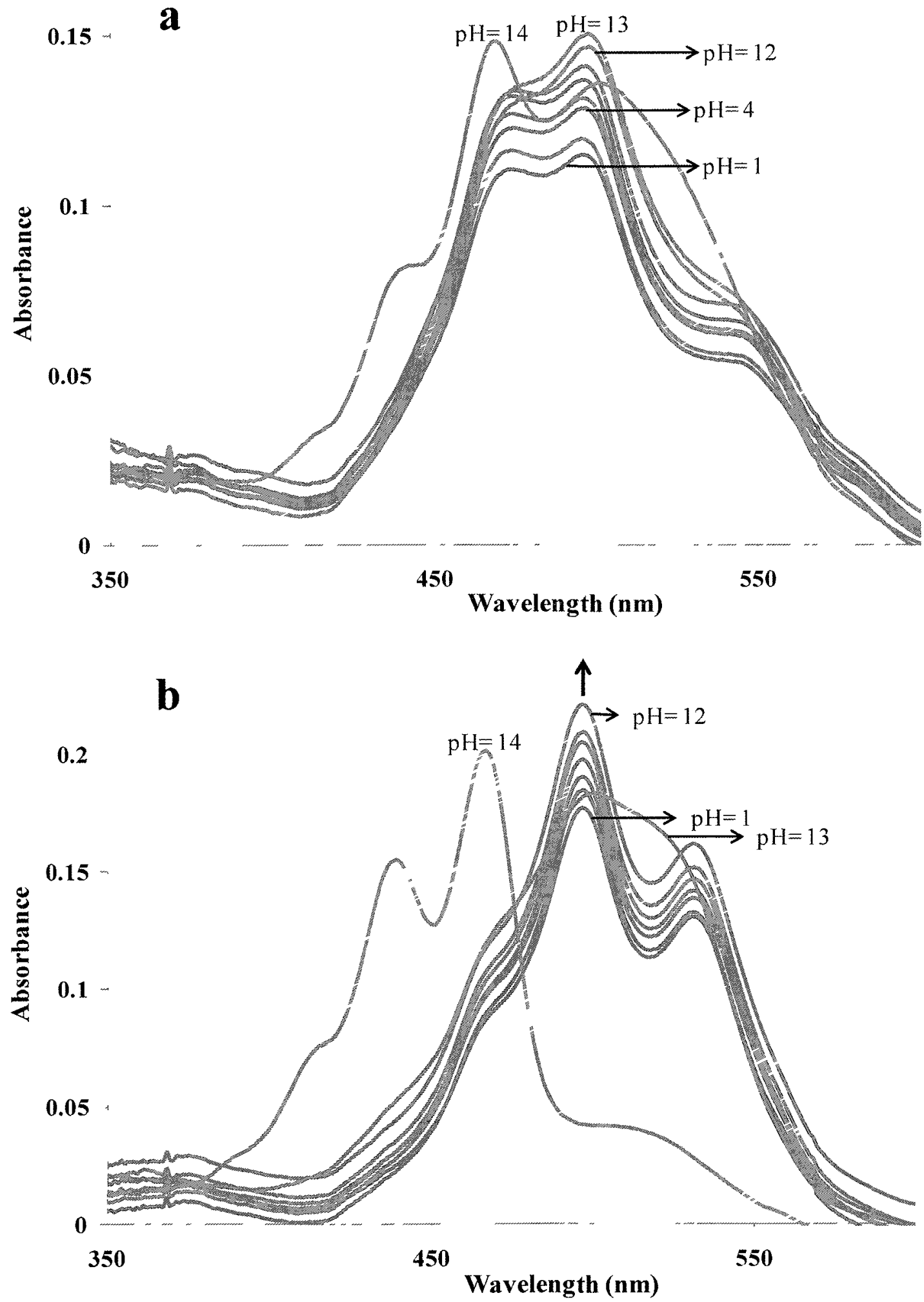

Figure 3.9: Absorption spectra of DJPTCDI (a) and MJPTCDI (b) at different $\mathrm{pH}$ at $10^{-5}$ M. 
The UV-Vis spectra, as a function of temperature, are shown in Figure 3.10, with water as well as acetone/water mixture as the solvent. With water, in the case of DJPTCDI a gradual change of intensity (either hyperchromic or hypsochromic shift) with a smaller blue shift is seen and the relative intensities of the $\mathrm{S}_{0-0}$ and $\mathrm{S}_{0-1}$ absorption bands (530 and $495 \mathrm{~nm}$, respectively) change with temperature, although there is no intensity reversal. The change in intensity between two vibronic transitions is very common in perylene diimide when the aggregate structure breaks down to monomer form. But Figure 3.10a shows that even at $90^{\circ} \mathrm{C}$ total intensity reversal didn't occur which confirms the strong stability of DJ-PTCDI in aggregated state. The gradual increase in intensity of the $\mathrm{S}_{0-0}$ transition and a decrease in the intensity of the $\mathrm{S}_{0-1}$ transition along with a small blue shift towards shorter wavelength (from 530 to $527 \mathrm{~nm}$ for $\mathrm{S}_{0-0}$ and from $495-492 \mathrm{~nm}$ for $\mathrm{S}_{0-1}$ transition) indicate the aggregate is disturbed but not destroyed. This shows that although Jeffamine, above its LCST of $30^{\circ} \mathrm{C}$ in water would be in a collapsed conformation, interpenetrating coils of Jeffamine on both sides of PTCDI still facilitate the $\pi-\pi$ interaction even at high temperatures. On the other hand, MJ-PTCDI shows intensity drop with a slight hypsochromic shift with temperature (figure $3.10 \mathrm{~b}$ ). The reversal occurs between $\mathrm{S}_{0-1}$ and $\mathrm{S}_{0-2}$ transition bands. Upon increasing the temperature of the solution, the peak at $547 \mathrm{~nm}$ for the $\mathrm{S}_{0-0}$ transition becomes less intense and the $\mathrm{S}_{0-1}$ transition peak gradually becomes a shoulder with a hypsochromic shift from 497 to 494 $\mathrm{nm}$ and $\mathrm{S}_{0-2}$ absorption band moves from 477 to $473 \mathrm{~nm}$ and becomes the most intense peak. 

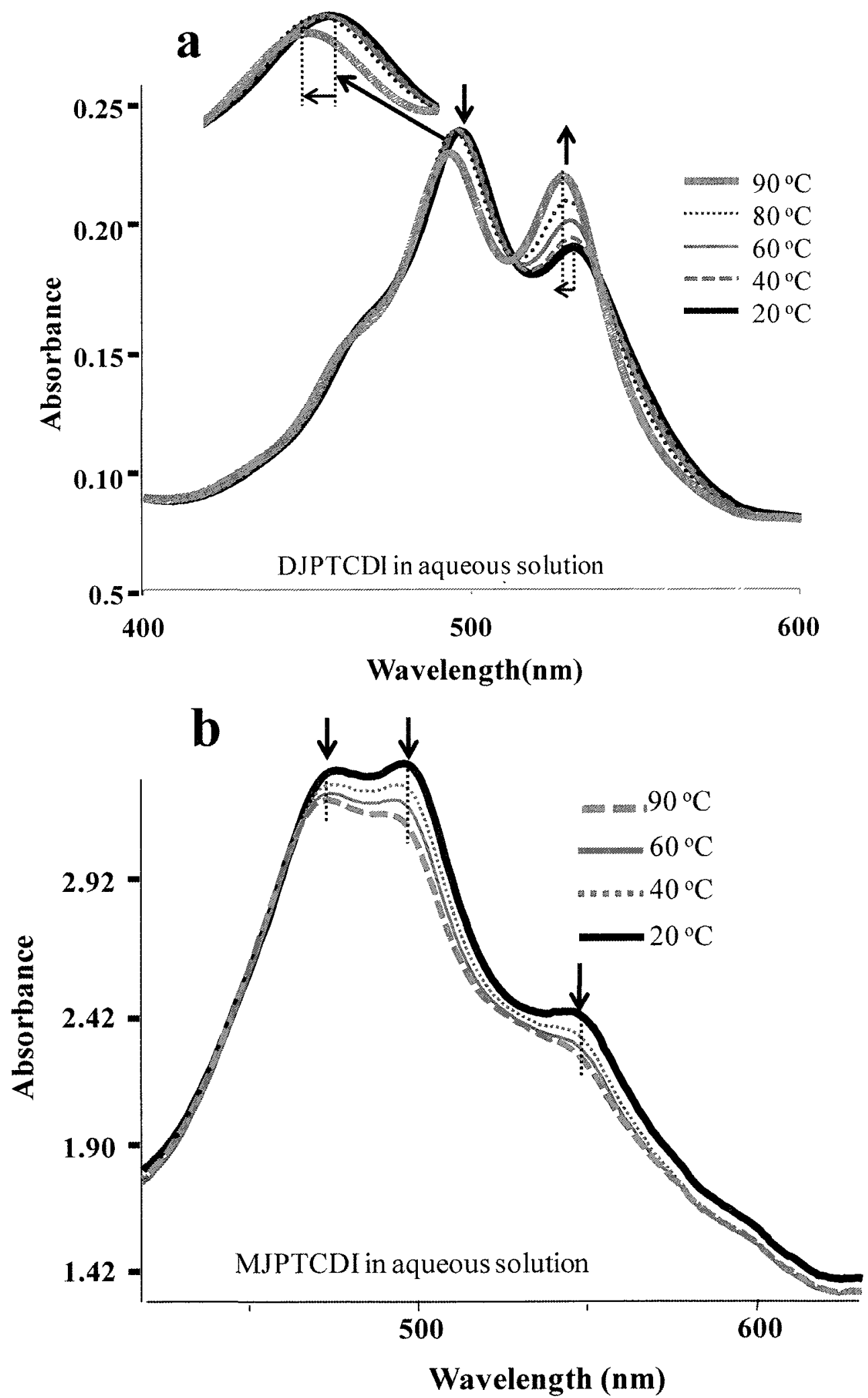

Figures continued in the next page 

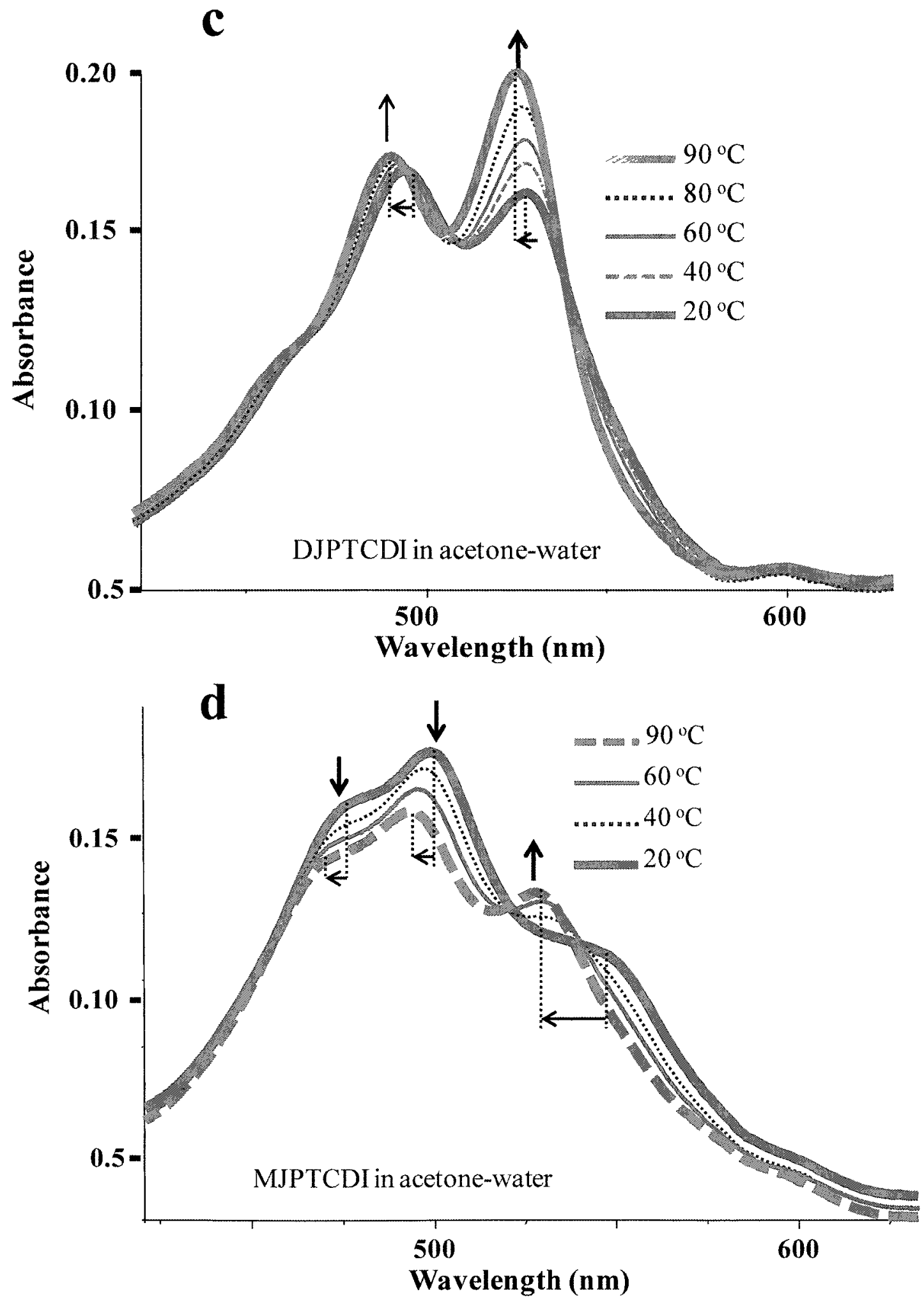

Figure 3.10: Variable temperature UV-Vis spectra of DJ-PTCDI (a) and MJ-PTCDI (b) in water and in acetone-water (1:9) mixture (c and d respectively). 
In a mixture of solvents the absorption pattern of DJ-PTCDI and MJ-PTCDI shows a different trend with temperature. With DJ-PTCDI solution in acetone/water (1/9) mixture, figure $3.10 \mathrm{c}$ shows a gradual increase in intensity of the $\mathrm{S}_{0-0}$ transition peak with temperature, over the $S_{0-1}$ peak with a very small $(\approx 3 \mathrm{~nm})$ hypsochromic shift. This confirms the slow dissociation of the aggregates to monomeric form. The transition occurs at $40^{\circ} \mathrm{C}$ indicating the weak nature of the aggregates in a mixture of solvents. The increased solubilization of the $\pi$-system in acetone with temperature causes the weakly stacked aggregate to dissociate. In the case of MJ-PTCDI a gradual evolution of a peak for 0-0 transition with a reduction of intensity of both $S_{0-1}$ and $S_{0-2}$ peaks is seen (figure 3.10d). Note that the shoulder-like peak for $\mathrm{S}_{0-0}$ transition exhibits a large blue shift from 548 to $528 \mathrm{~nm}$ and the $\mathrm{S}_{0-1}$ and $\mathrm{S}_{0-2}$ transition peaks show a slight shift towards shorter wavelength.

Figure 3.11 shows the plot of variation of $\alpha_{\text {agg }}(\mathrm{T})$ with temperature for DJ-PTCDI and MJ-PTCDI, calculated using equation 3.1. It is seen that $\alpha_{\text {agg }}=0.5$ is reached at about $40^{\circ} \mathrm{C}$ for DJ-PTCDI whereas MJ-PTCDI shows exceptional stability and remains associated even at $90^{\circ} \mathrm{C}$. These observations suggest that the aggregates begin to dissociate at elevated temperatures although the stability is different for DJ-PTCDI and MJ-PTCDI.

NMR is another technique used to study the self-assembly of $\pi$-stacked aromatic chromophores in solution. ${ }^{31-37}$ The ring current of a chromophore induces the resonance frequency of the proton of a neighboring chromophore. ${ }^{31,32}$ Normally, an upfield 
chemical shift indicates $\pi$-stacked association. ${ }^{31}$ Figure 3.12 shows the ${ }^{1} \mathrm{H}$ NMR spectra for DJ-PTCDI and MJ-PTCDI in $\mathrm{CDCl}_{3}$ and $\mathrm{D}_{2} \mathrm{O}$. Both of them show the characteristic multiplet of perylene unit in $\mathrm{CDCl}_{3}$ confirming their presence as non-stacked monomeric form in non-aqueous solvents (which was confirmed by UV-Vis spectra). But in water, DJ-PTCDI shows an upfield peak ${ }^{35.36}$ with a shoulder. The upfield shift confirms the stacking of DJ-PTCDI in water. ${ }^{34.37 .38} \mathrm{~A}$ gradual downfield shift of this peak with increasing temperature indicates the disintegration of the aggregate. As seen from UVVis spectra, a certain minimum concentration is required for aggregation of DJ-PTCDI in water (an intensity reversal at $10^{-7} \mathrm{M}$ ) and it breaks up gradually with temperature. We could not record a spectrum at the low concentration of $10^{-7} \mathrm{M}$. In case of MJ-PTCDI also we see perylene multiplet with non aqueous solvent, but an upfield shift in water. With an increase in temperature, the peak shifts downfield as the aggregate is destabilized. 


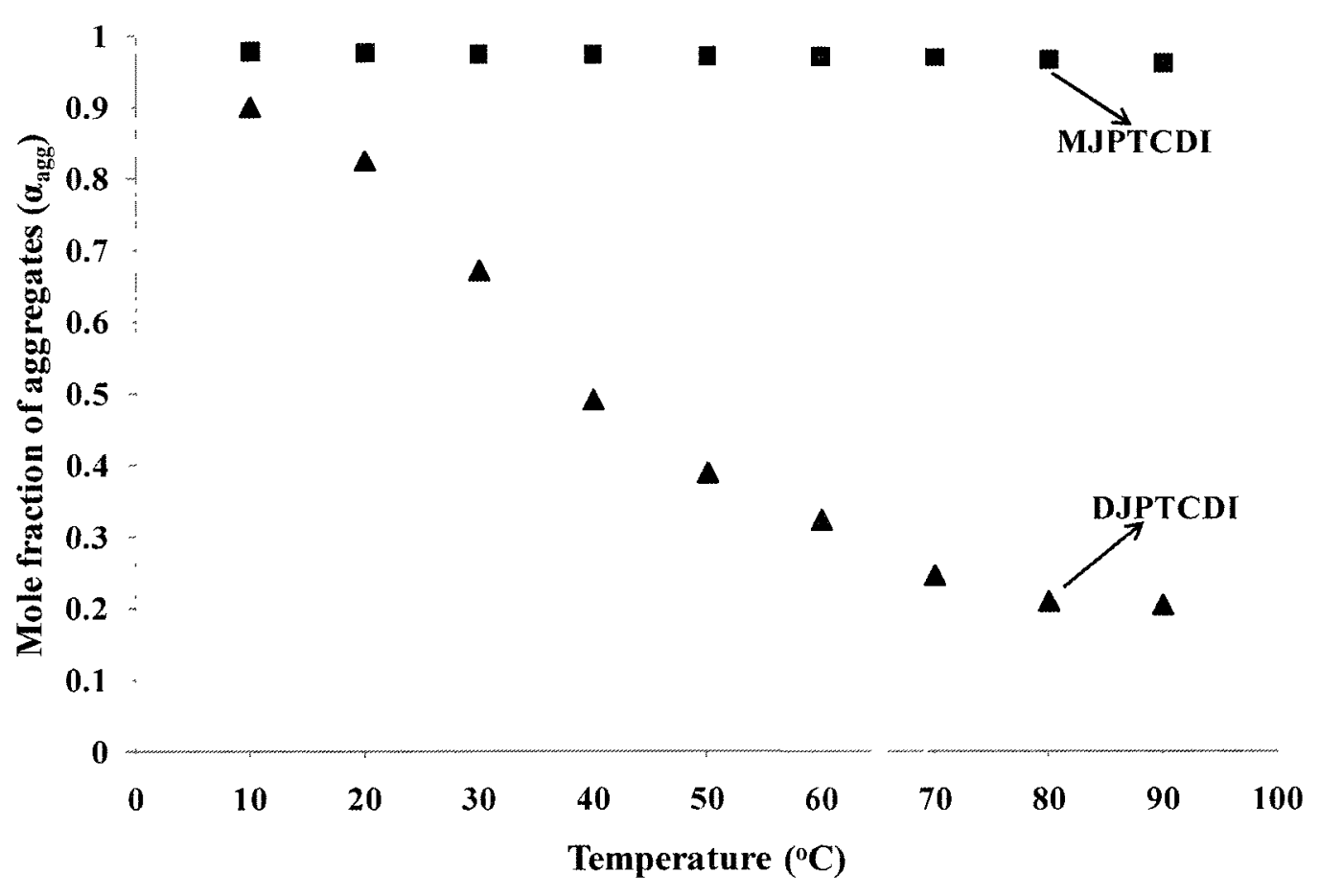

Figure 3.11: Mole fraction of aggregates vs temperature curve for MJ-PTCDI and DJPTCDI in acetone-water (1:9) mixture. 

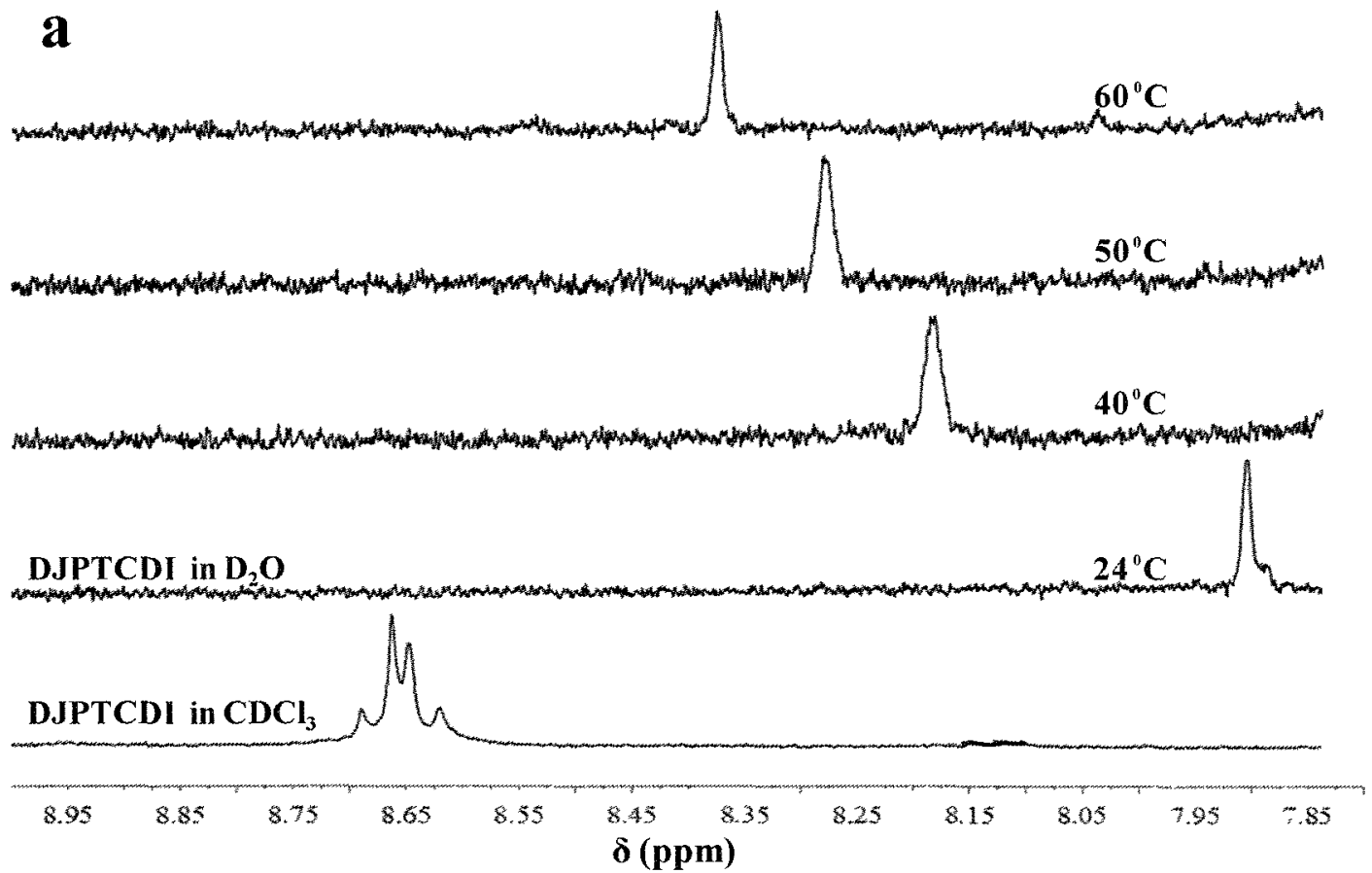

b

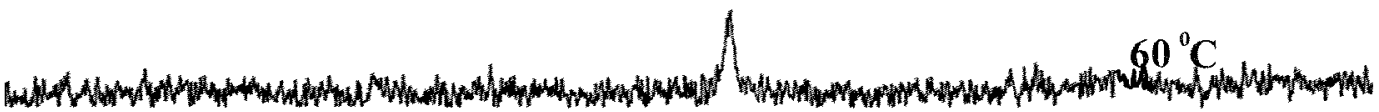

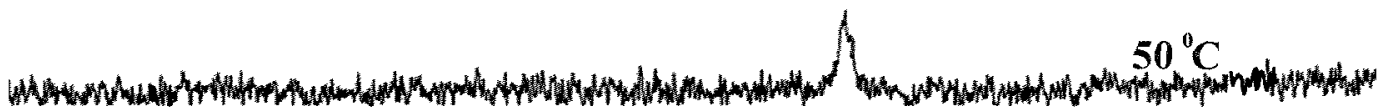

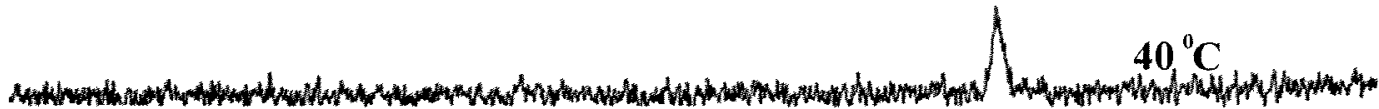

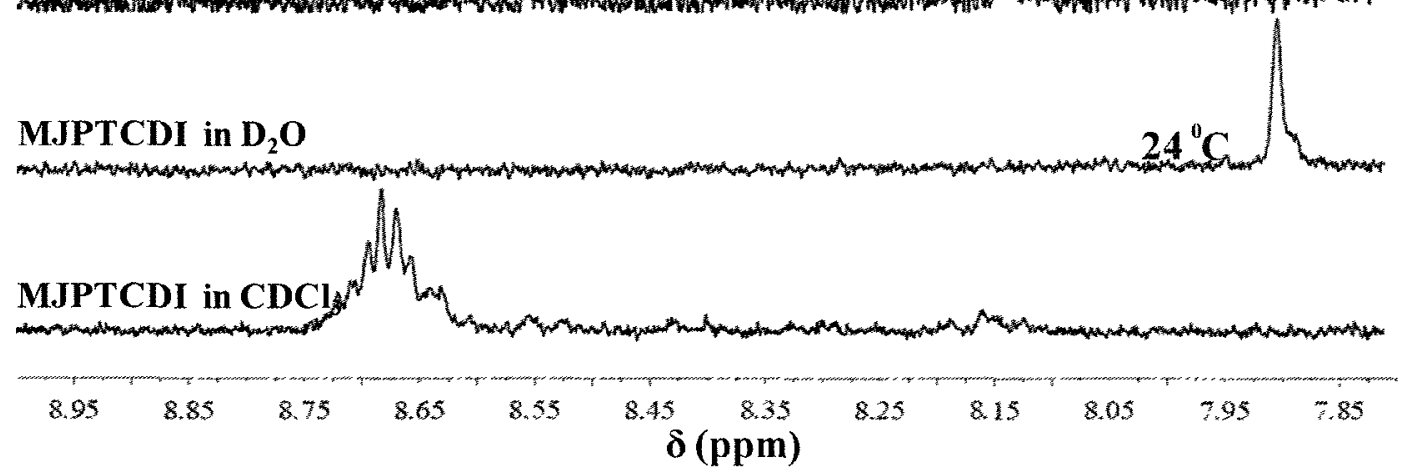

Figure 3.12: ${ }^{1} \mathrm{HNMR}$ spectra of DJ-PTCDI (a) and MJ-PTCDI (b) in $\mathrm{CDCl}_{3}$ and at various temperatures in $\mathrm{D}_{2} \mathrm{O}$. 


\subsubsection{Microscopy}

Figure 3.13 shows the OM of as-prepared samples of DJ-PTCDI and MJ-PTCDI as well as samples that were drop-cast from $10^{-4} \mathrm{M}$ aqueous solutions. The aggregates due to perylene are seen in both cases. This indicates that even in the bulk with the polymer attached, there is self-assembly of the perylene molecules into crystals, in the polymer matrix. The UV-Vis spectra of the solid samples of DJ-PTCDI and MJ-PTCDI are shown in figure 3.4c. Over 30 years ago, Dulmage et. $\mathrm{al}^{28}$ discussed the beneficial effect of such aggregated structures on the photoconduction properties of aryl- substituted thiapyrylium salt. Such aggregation broadens the spectral response, as was mentioned above with respect to Figure 3.6. The freeze-dried samples of DJ-PTCDI and MJ-PTCDI consist of spherical aggregates as seen from the scanning electron micrographs shown in Figures 3.13e and 3.13f, for $10^{-3} \mathrm{M}$ concentration. These are $10-20$ and 5-15 $\mu \mathrm{m}$ in diameter for DJ-PTCDI and MJ-PTCDI, respectively. This size range is also confirmed by the dynamic light scattering data shown in figure 3.14.

We found that the morphology changed significantly, upon ageing of the solutions for a few months. Figure 3.15 shows that long fibers have formed. The birefringence of the fibers confirms the presence of crystalline aggregates. UV-Vis spectra of these aged solutions showed that the aggregates still remained, and there was no indication of 
transformation to monomeric form. We believe that the aggregates seen in figure 3.13 simply coalesced to form these fibers. The slightly enlarged part of the fiber in figure $3.15 \mathrm{~d}$ shows that it is composed of individual aggregates. It is interesting that the coalescence is directional as to form fibers due to the packing of successive stacked aggregates of the perylene units, and not large spherical aggregates. Similar fiber formation was seen upon ageing, with solutions of $\mathrm{pH}=6$, as shown in figure 3.16 . 

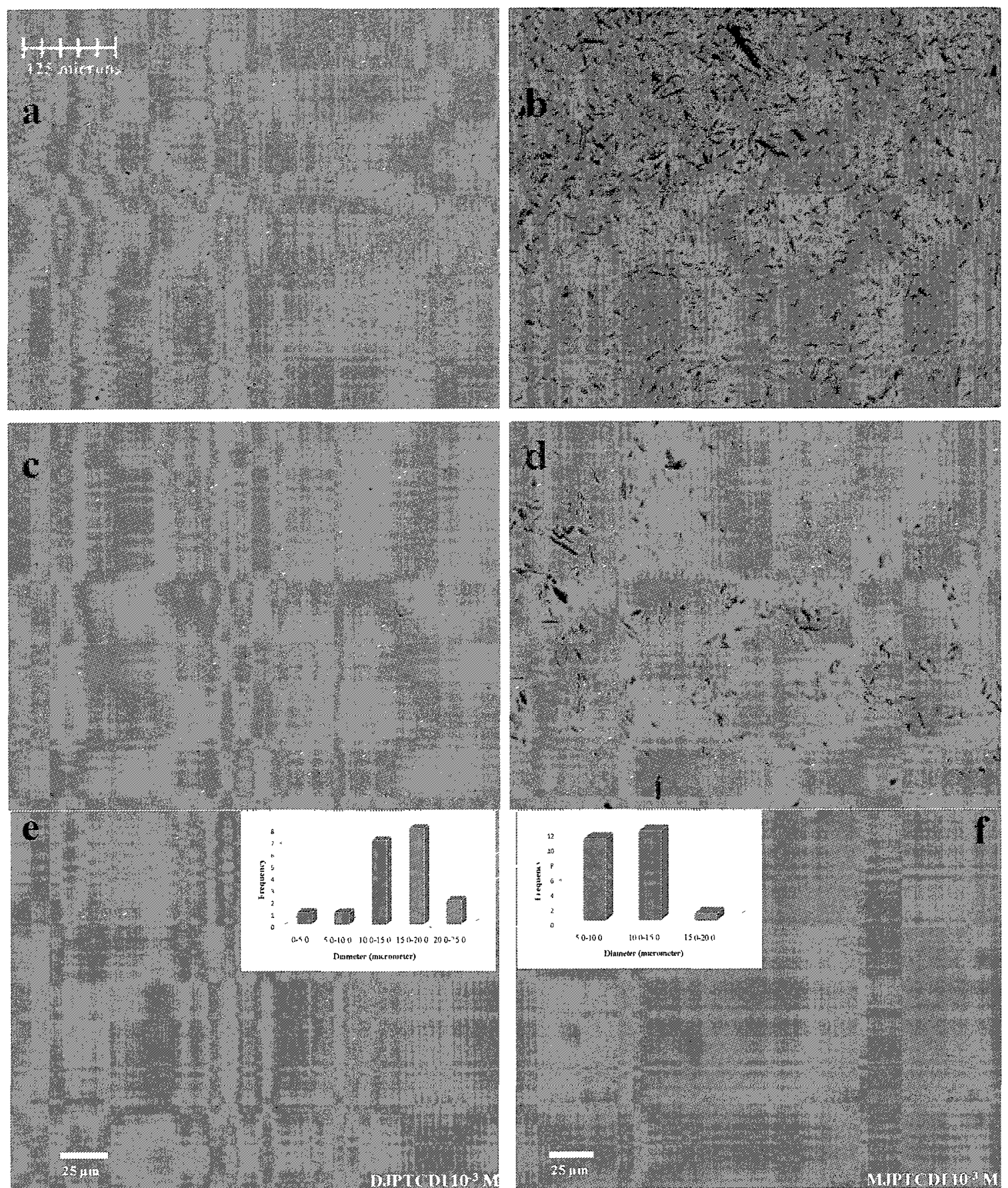

Figure 3.13: (a) DJ-PTCDI as is (b) MJ-PTCDI as is (c), DJ-PTCDI drop-cast (d) MJPTCDI drop-cast (e) SEM of DJ-PTCDI freeze-dried and (f) SEM of MJ-PTCDI freezedried. Here $a, b, c$, and d are of same scale. 


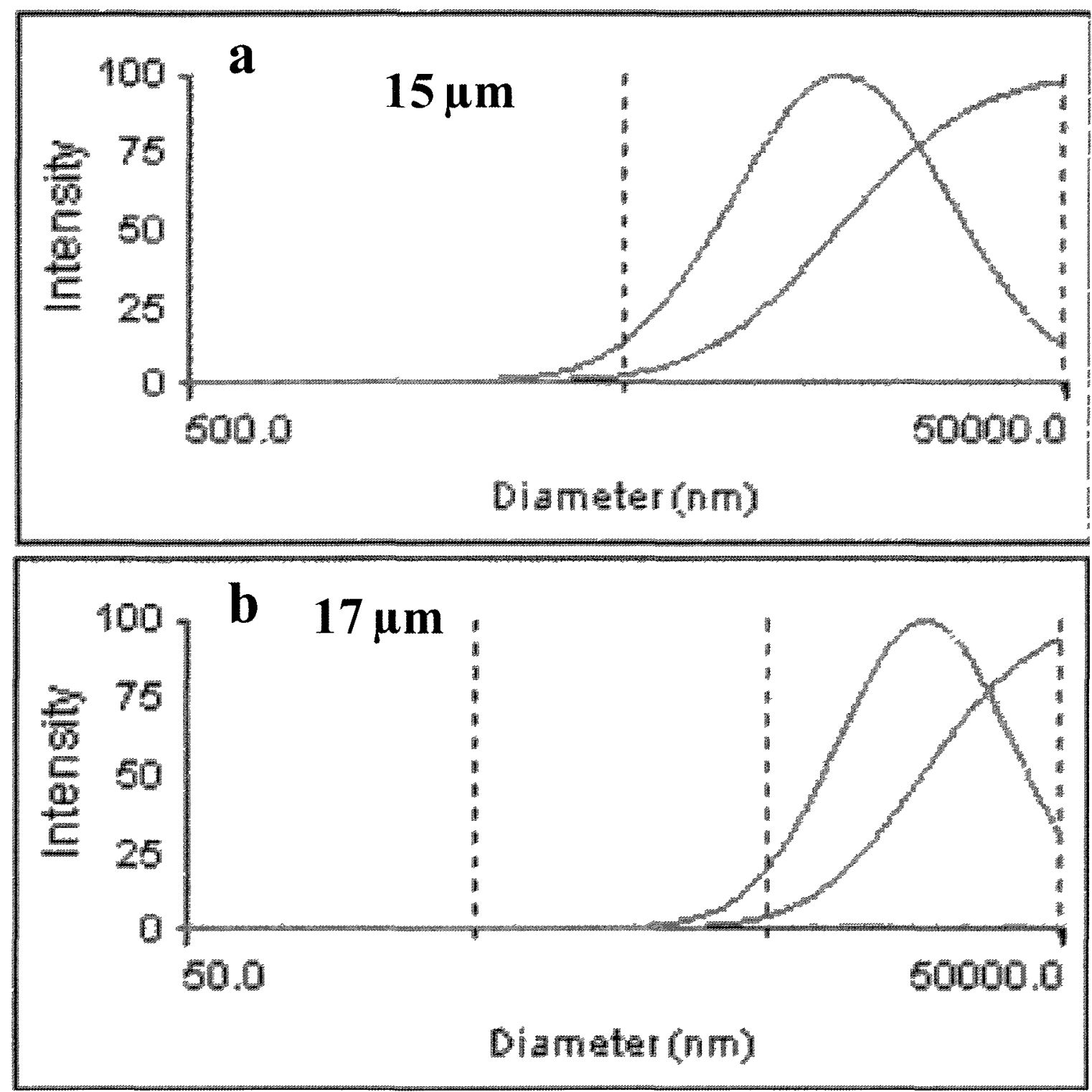

Figure 3.14: Log normal distribution of the DLS data of (a) DJPTCDI and (b) MJPTCDI in water at $10^{-4} \mathrm{M}$ concentration. 

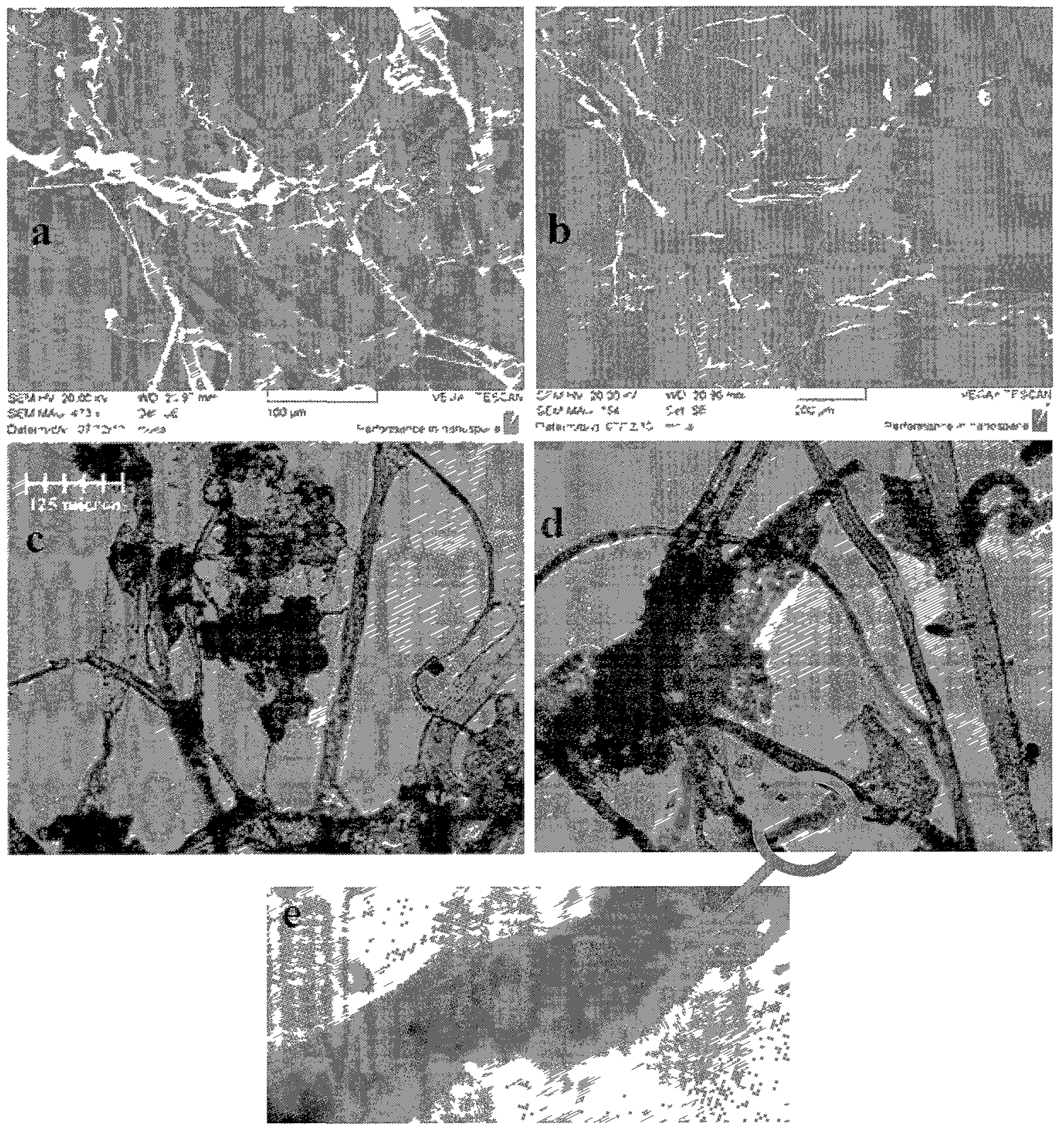

Figure 3.15: SEM and OM of drop-cast films of aged aqueous solutions of DJ-PTCDI (a and c) and MJ-PTCDI (b and d). OM images $c$ and d are of same scale. An enlargement of a part of (d) is shown in (e). 

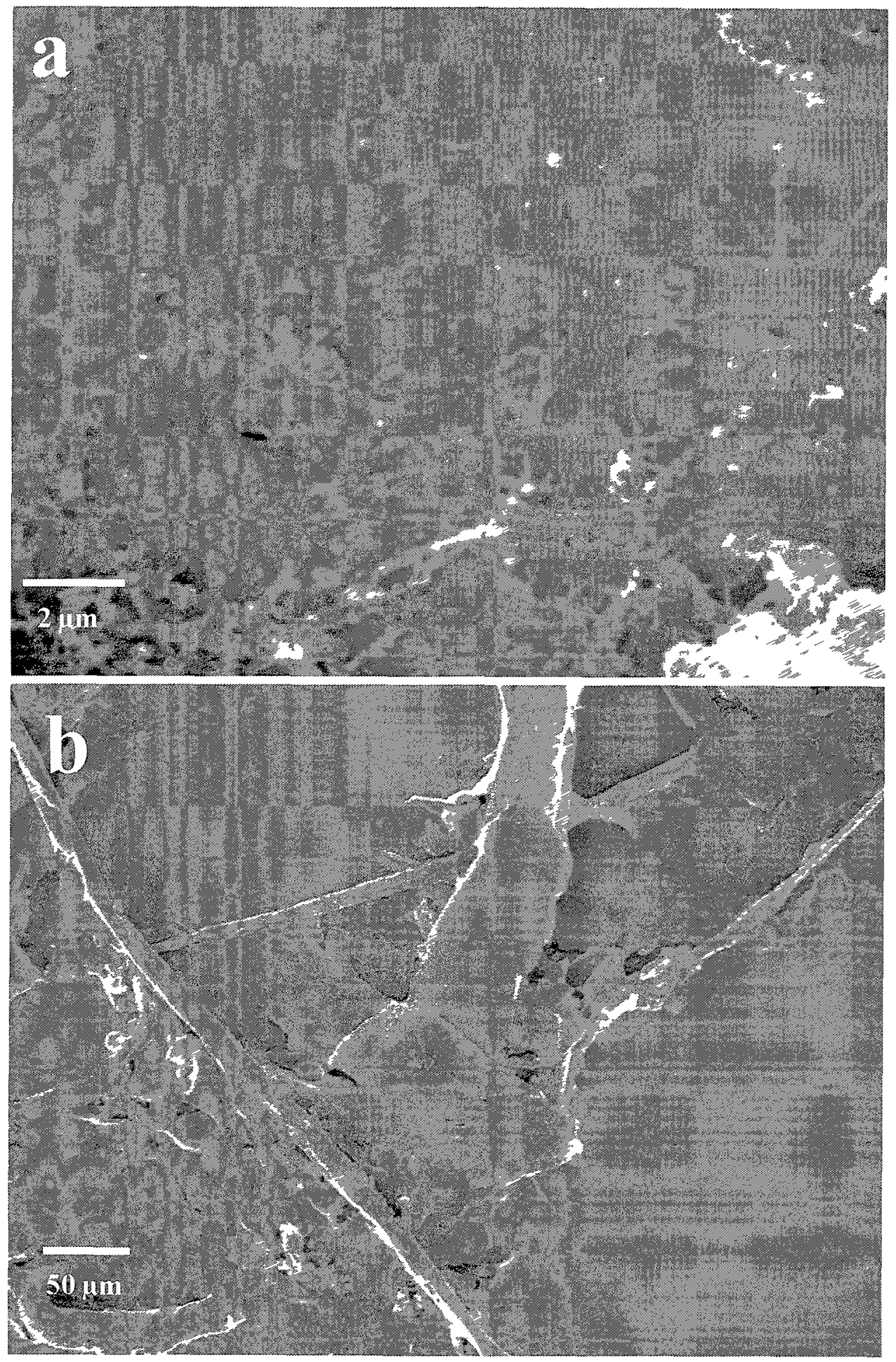

Figure 3.16: SEM Images of drop-cast films of DJ-PTCDI (a) and MJ-PTCDI (b) from an aged $\mathrm{pH}=6$ solution. 
We discussed above that only the monomeric form was indicated in the UV-Vis spectra of DJ-PTCDI and MJ-PTCDI in non-aqueous solvents, as seen in figure 3.4. The optical and SEM micrographs of drop-cast samples (from $10^{-4} \mathrm{M}$ solutions) from various solvents are shown in figures 3.17 and 3.18 for DJ-PTCDI and MJ-PTCDI. respectively. Although no aggregation of the chromophore occurred in solution, upon drying, needlelike crystalline aggregates are seen in all cases. The sample from THF shows an interesting treed morphology, with fibers and branches. The corresponding SEM shows Lego-like blocks aggregating predominantly in one direction to form fiber-like morphology. While nanofibers and discotic assemblies were seen with short substituents, the flexible polymer Jaffamine (which is an ethylene oxide-propylene oxide copolymer) causes local aggregation of PTCDI and coalescence of these domains into such morphologies as shown in figures 3.17 and 3.18 . 

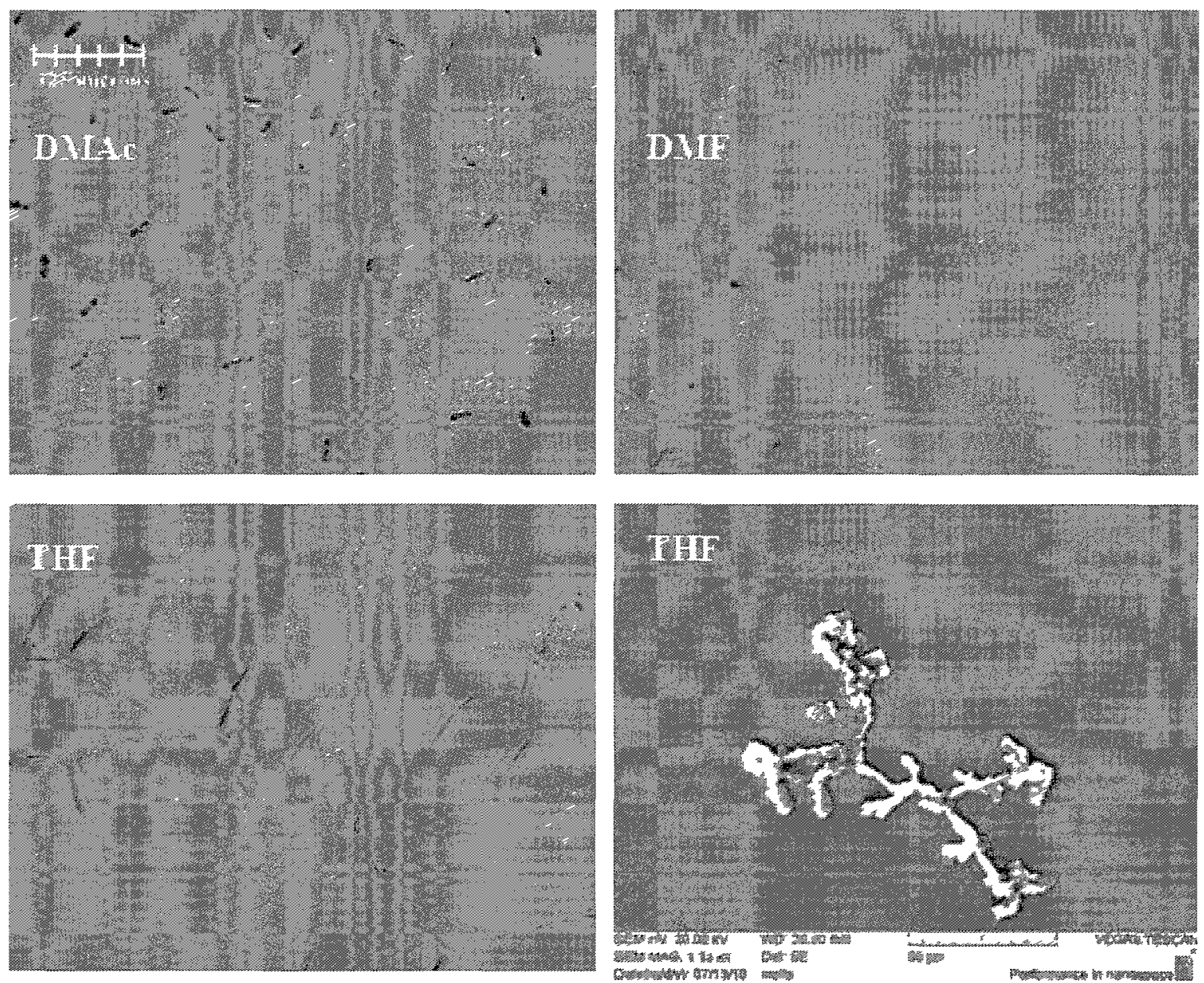

Figure 3.17: Optical and Scanning electron micrographs of drop-cast solutions of DJPTCDI from various solvents. All the OM images are of same scale. 

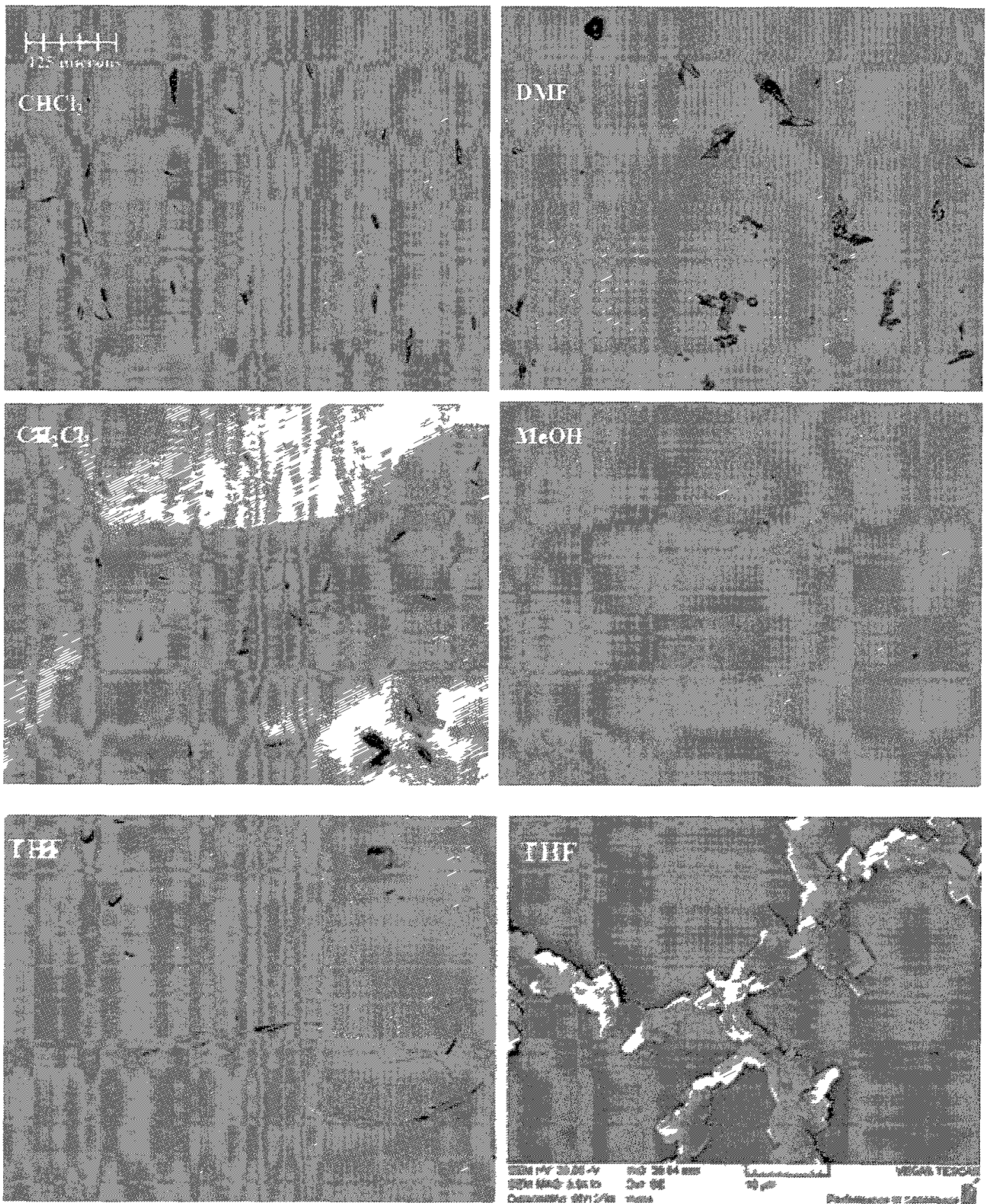

Figure 3.18: Optical and Scanning electron micrographs of drop-cast solutions of MJPTCDI from various solvents. All the OM images are of same scale. 


\subsubsection{Thermal Analysis}

The DSC scans of DJ-PTCDI and MJ-PTCDI are shown in figure 3.19. Unlike the perylene diimides with short chain substituents, DJ-PTCDI and MJ-PTCDI with the polymer did not show any liquid crystalline behavior. Our previous work ${ }^{21,22}$ on the PDMS based perylenediimide pseudo block copolymers also showed no LC transitions. Thus long chains attached to perylene seem to inhibit the LC behavior. The melting temperatures of perylene diimides are in the range of $300{ }^{\circ} \mathrm{C}$. Figure 3.19 shows an irreversible transition at $87{ }^{\circ} \mathrm{C}$ for DJ-PTCDI and at $122{ }^{\circ} \mathrm{C}$ for MJ-PTCDI. Similar transitions in this temperature range have been reported by other authors ${ }^{24.39}$ for perylenediimides substituted with short chains. Apart from mentioning this transition, its origin has not been discussed. We recorded X-ray diffraction and UV-Vis spectra after annealing the sample for an hour at a temperature above these transitions. However, there was no difference in the results that would indicate a polymorphic transition. Thus, the origin of the transition in this temperature range remains unknown. 

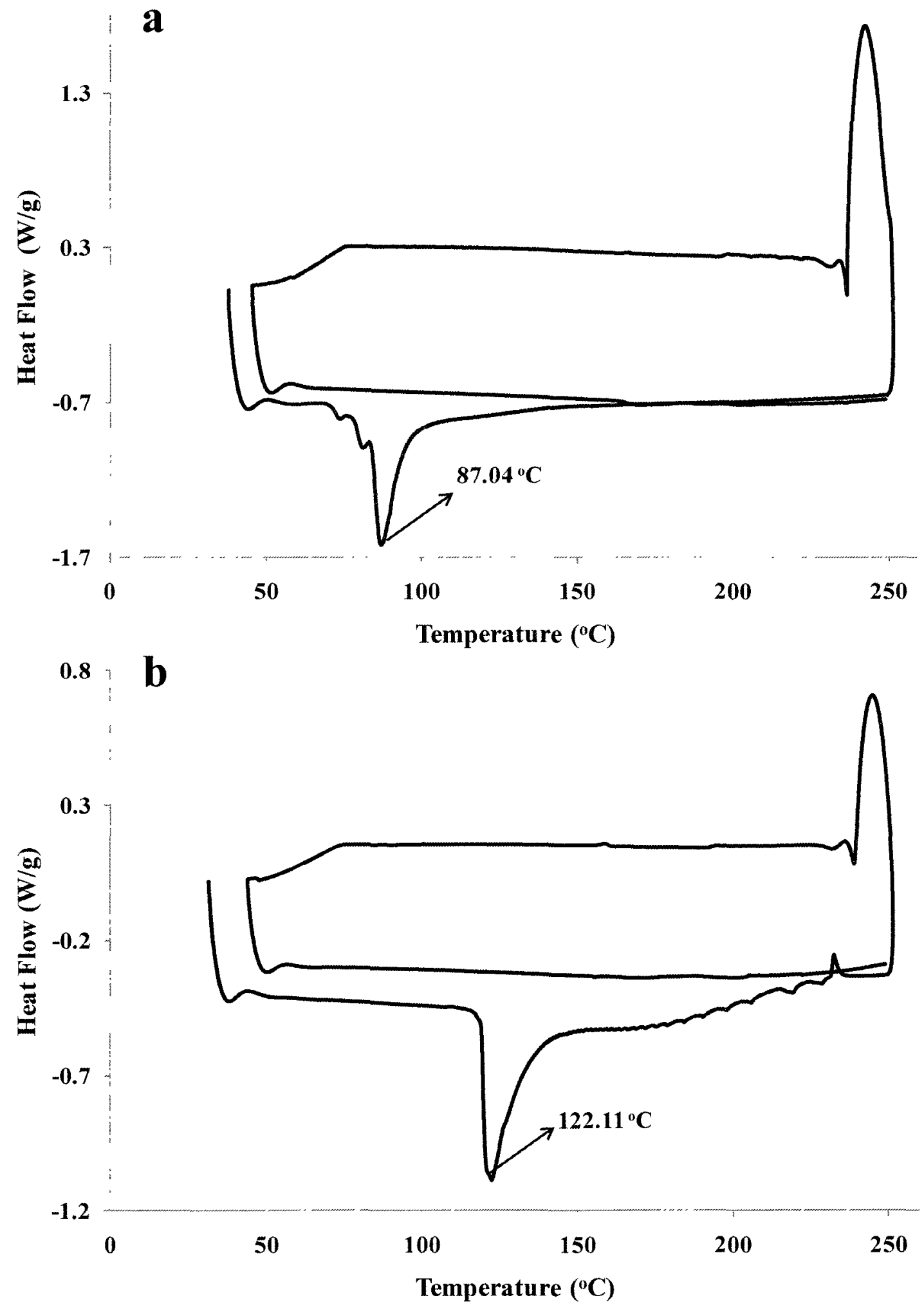

Figure 3.19: DSC of (a) DJ-PTCDI and (b) MJ-PTCDI. 


\subsubsection{X-Ray Analysis}

The X-ray diffraction traces of MJ-PTCDI and DJ-PTCDI are shown in the figure 3.20. Both MJ-PTCDI and DJ-PTCDI showed a broad peak from $2 \theta=15$ to $25^{\circ}$. The amorphous hump is due to Jeffamine. The d-spacings are different between DJ-PTCDI $(9.30,7.13,3.59$ and $3.24 \AA)$ and MJ-PTCDI $(9.61,6.83$ and $6.07 \AA$, and 2.97). The 3.24 $\AA$ spacing can be attributed to $\pi$ - stacking. Although a similar spacing was not clearly seen in the MJ-PTCDI diffraction, there is considerable noise in that region. The rest of the reflections indicate that the packing in these cases are different. 

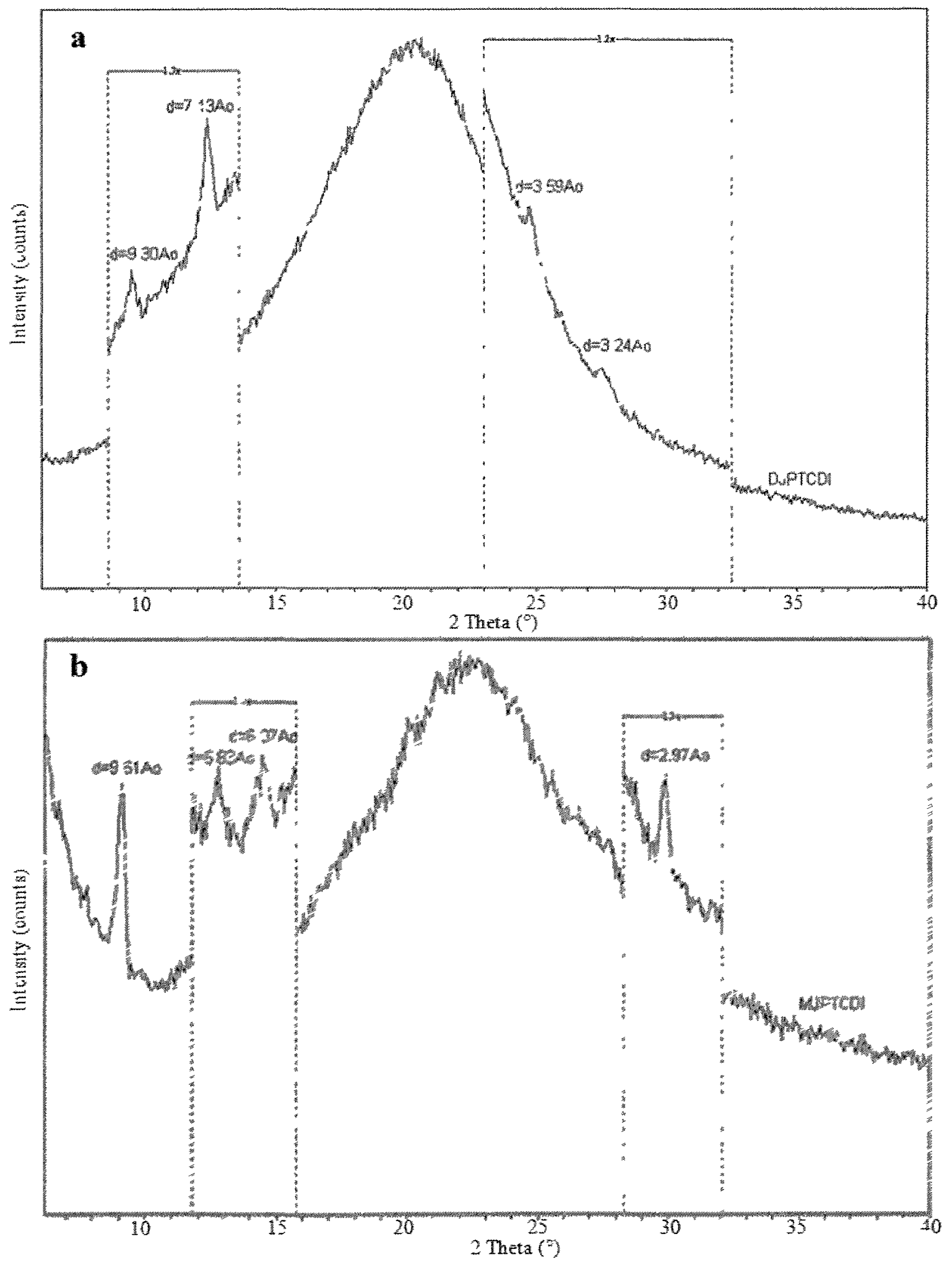

Figure 3.20: X-ray diffraction profile of (a) DJ-PTCDI and (b) MJ-PTCDI. 


\subsection{Conclusions}

We described the self-assembly of rod-coil polymers with the large aromatic chromophore, perylenebisimide as the rod segment, with flexible Jeffamine attached to one (MJ-PTCDI) or both sides (DJ-PTCDI). The self-assembly and aggregation of the chromophore has been examined by UV-Vis and NMR spectra, microscopy and X-ray diffraction. While there is a vast literature on the amphiphilic block copolymers with a hydrophilic segment such as PEG, and their use in various applications including $\mathrm{pH}$ dependent delivery of drug molecules, we have shown that such amphiphilic systems can be designed with photoconductors (as applicable to optoelectronics) as part of a rod-coil copolymer. The di-block and triblock copolymers with perylenebisimide and Jeffamine enable the aggregation of the chromophores in aqueous media. We have shown that the mode of aggregation is different between MJ-PTCDI and DJ-PTCDI, and that the extent of aggregation can be modified by solvent mixtures e.g., acetone/ water. Because of the solubility of the polyetheramine in water in a broad range of $\mathrm{pH}$, the aggregation of perylenebisimide in water remains up to a $\mathrm{pH}$ of 12.4 . Ageing of the solutions leads to predominantly one-dimensional coalescence into fibers. The aggregation also shows thermal stability, as seen from UV-Vis and NMR spectra. In addition, due to the solubility in water and good fluorescence, this is likely to be an interesting material for use as fluorescence probes in biological applications. 


\subsection{References}

1. He, L.; Zhang, L.; Ye, Y.; Liang, H. J. Phys. Chem. B 2010, 114, 7189.

2. Chang, W.-H.; Chou, S.-H.; Lin, J.-J.; Chen, W.-C.; Sheng, Y.-J. J. Chem. Phys. 2010, $132,214901(1-14)$.

3. Koh, H.-D.; Changez, M.; Rahman, M. S.; Lee, J.-S. Langmuir 2009, 25, 7188.

4. Olsen, B. D.; Segalman, R. A. Mater. Sci. Eng. Part R 2008, 62, 37.

5. Rahman, M. S.; Samal, S.; Lee, J.-S. Macromolecules 2007, 40, 9279.

6. Rahman, M. S.; Samal, S.; Lee, J.-S. Macromolecules 2006, 39, 5009.

7. Discher, B. M.; Won, Y.-Y.; Ege, D. S.; Lee, J. C-M.; Bates, F. S.; Discher, D. E.;

Hammer, D. A. Science 1999, 287, 1143

8. Jenekhe, S. A.; Chen, X. L. Science 1998, 279, 1903.

9. Park, J.-W.; Thomas, E. L. Macromolecules 2004, 37, 3532.

10. Lee, M.; Cho, B.-K.; Zin, W.-C. Chem. Rev. 2001, 101, 3869.

11. Chen, J. T.; Thomas, E. L.; Ober, C. K.; Hwang, S. S. Macromolecules 1995, 28, 1688.

12. (a) Astafieva I.; Zhong, X.; Eisenberg, A. Macromolecules 1993, 26, 7339. (b) Zhang, L.; Eisenberg, A. Science 1995, 268, 1728. (c) Zhang, L.; Eisenberg, A. J. Am. Chem. Soc. 1996, 118, 3168. (d) Zhang, L.; Yu, K.: Eisenberg, A. Science 1996, 272, 1777. 
13. (a) Wang, X.S.; Winnik, M.A.: Manners, I. Macromol Rapid Commun. 2002, 23. 210.

(b) Raez, J.; Manners. I.; Winnik, M. A. J. Am. Chem. Soc. 2002, 124, 10381.

14. (a) Kim, B.-S.; Hong, D.-J.; Bae. J.; Lee, M. J. Am. Chem. Soc. 2005, 127, 16333. (b)

Jiang, L.; Hughes, R. C.; Sasaki, D. Y. Chem. Commun. 2004,8,1028. (c) Wang, H.;

Wang, H. H.;Urban. V. S.; Littrell, K. C.; Thiyagarajan, P.; Yu, L. J. Am. Chem. Soc. 2000, 122, 6855. (d) Lee, M.; Jang C.-J.; Ryu, J.-H. J. Am. Chem. Soc. 2004, 126, 8082.

15. (a) Balakrishnan, K.; Datar, A.; Naddo, T.; Huang, J.; Oitker, R.; Yen, M.; Zhao, J.; Zang, L. J. Am. Chem. Soc. 2006, 128, 7390. (b) Briseno, A. L.; Mannsfeld, S. C. B.; Reese, C.; Hancock. J. M.; Xiong, Y.; Jenekhe, S. A.; Bao, Z.; Xia, Y. Nano Lett. 2007, 7, 668. (c) Ryu, J-H. ; Hong, D-J.; Lee, M. Chem. Commun 2008, 9, 1043.

16. Guan, Y.; Yu S., H.: Antonietti, M.; Bçttcher, C.; Faul, C. F. J. Chem. Eur. J. 2005, 11,1305 .

17. Schmidt-Mende, L.; Fechtenkotter, A.; Mullen, K.; Moons, E.; Friend, R. H.; MacKenzie, J. D. Science 2001, 293, 1119.

18. Gregg. B. A. J. Phys. Chem. 1996. 100, 852.

19. (a) Yang, X.; Xu. X.; Ji, H.-F. J. Phys. Chem. B 2008, 112, 7196. (b) Zang, L.; Che, Y.; Moore, J.S. Accounts Chem. Res. 2008,41,1596. (c) Chen, Z.; Stepanenko, V.; Dehm, V.; Prins. P.; Siebbeles, L.D.A.: Seibt, J.; Marquetand, P.; Engle, V.; Wurthner, F. Chem. Eur. J. 2007,13, 436. (d) Zhang, X.; Chen, Z.: Wurthner, F. J. Am. Chem. Soc. 2007, 129, 
4886. (e) Gregg, B. A.; Cormier, R. A. J. Am. Chem. Soc. 2001, 123, 7959. (f) Struijk, C.W.; Sieval, A. B.; Dakhorst, J. E. J.; van Dijk, M.; Kimkes, P.; Koehorst, R. B. M.; Donker, H.; Schaafsma, T. J.; Picken, S. J.; van de Craats, A. M.; Warman, J. M.; Zuilhof, H.; Sudholter, E. J. R. J. Am. Chem. Soc. 2000, 122, 11057. (g) Li, A. D. Q.;

Wang, W.: Wang, L.-Q. Chem. Eur. J. 2003, 9, 4594. (h) Cormier, R. A.; Gregg, B. A. Chem. Mater. 1998, 10, 1309.

20. Yao, D.; Wang, Z. Y.; Sundararajan, P. R. Polymer 2005, 46, 4390.

21. Yao, D.; Bender, T. P.; Gerroir, P. J.; Sundararajan, P. R. Macromolecules 2005, 38, 6972.

22. Yao, D.;Tuteja, B.; Sundararajan, P. R. Macromolecules 2006, 39, 7786.

23. Nagao Y.; Misono T. Bull.Chem.Soc. Jpn. 1981, 57,1191.

24. Chen, Z.; Baumeiste, U.; Tschierske, C.; Wurthner, F. Chem. Eur. J. 2007, 13, 450.

25. Wurthner, F.; Chen, Z.; Hoeben, F. J. M.; Osswald, P.; You, C.-C.; Jonkheijm, P.; Herrikhuyzen, J. v.; Schenning, A. P. H. J.; van der Schoot, P. P. A. M.; Meijer, E. W.;

Beckers, E. H. A.; Meskers, S. C. J.; Janssen, R. A. J. J. Am. Chem. Soc. 2004, 126 , 10611.

26. Langhals, H.; Jona, W. Angew. Chem., Int. Ed. 1998, 37, 952.

28. Dulmage, W. J.; Light, W. A.; Marino, S. J.; Salzberg, C. D.; Smith, D. L.; Staudenmayer, W. J. J. Appl. Phys. 1978, 49, 5543. 
29. Arnaud. A.; Belleney, J.; Boue, F.; Bouteiller, L.; Carrot, G.; Wintgens, V. Angew. Chem. Intl. Ed. 2004, $\$ 3,1718$.

30. Ghosh S.; Li, X.-Q.; Stepanenko, V.; Wurthner, F. Chem. Eur. J. 2008, 14, 11343.

31. Li, A. D. Q.; Wang, W.; Wang, L.-Q. Chem. Eur. J. 2003, 9, 4594.

32. Shaller, A. D.; Wang. W.; Gan, H.; Li, A. D.Q. Angew Chem. Intl. Ed. 2008. 47, 1.

33. Li, Y.; Li, Y.; Li. J.; Li, C.; Liu, X.; Yuan, M.; Liu, H.; Wang. S. Chem. Eur. J. 2006, 12,8378 .

34. Alvino, A.; Franceschin. M; Cefaro. C.; Borioni, S.; Ortaggia, G.; Bianco, A. Tetrahedron 2007, 63, 7858.

45. Martin, R. B. Chem. Rev. 1996, 96. 3043.

36. Nelson, J. C.; Saven, J. G.; Moore. J. S.; Wolynes, P. G. Science 1997, 277, 1793.

37. Lahiri, S.; Thompson, J. L.; Moore, J. S. J. Am. Chem. Soc. 2000, 122, 11315.

38. Giessner-Prettre, C.; Pullman. B.; Borer, P. N.; Kan, L.-S.; Ts'o, P. O. Biopolymers 1976, 15, 2277.

39. Pasaogullari, N.; Icil, H; Demuth, M. Dyes and Pigments 2006, 69, 118. 


\section{CHAPTER IV}

Tuning the Morphology of Amphiphilic Pseudo Block Copolymer of Perylene From Folded Sheet to Tube by Modulating the Hydrophobic Chain Length. 


\subsection{Introduction}

The science and technology of creating thermodynamically stable supramolecular structures by using the self-assembly process which involves spontaneous association of molecule via non-covalent interactions such as hydrogen bonding, $\pi-\pi$, van der Walls interactions etc. is maturing. The process of self-assembly is not new. Nature itself uses self assembly in every step of its creations. Molecular self-assembly is a strategy for nanofabrication that involves designing molecules and supramolecular entities with different functionalities. Normally, this may be a single step or a multi level organization to give a specific final structure. Several organic systems have been found which selforganize into nano structures including cyclic peptides, ${ }^{1,2}$ amphiphilic peptides, ${ }^{3,4}$ crowded aromatics, ${ }^{5-8}$ hexa-peri-hexabenzocoronenes, ${ }^{9,10}$ porphyrins, ${ }^{11-13}$ polyanilines, ${ }^{14-}$ ${ }^{16}$ phthalocyanines, ${ }^{17}$ cyanines, ${ }^{18,19}$ and merocyanines. ${ }^{20-22}$ The assembly of organic molecule needs the driving force from the molecules themselves by self assembling codes. Solvents play a vital role for self assembly as well. Molecular level self assembly includes hydrophobic and hydrophilic effects, electrostatic interactions, hydrogen bonding, micro phase segregation, and shape effects etc.

The self assembly of aromatic rod like molecules with flexible attachments has been discussed by Ryu et.al. ${ }^{22 b}$ Perylene diimides have strong, well-defined $\pi-\pi$ interactions which can be used to direct self-organization in various forms. Perylene diimide shows liquid crystalline (LC) ${ }^{23-24}$ behavior and gains application in electronics as transistors. ${ }^{25-31}$ Other potential applications include solar cells due to high quantum yield and thermal and photolytic stability. Perylene diimides show n-type semiconductor 
32-34 properties whereas the other organic functional materials show p-type semiconducting properties. But the poor solubility of perylene based system is a great problem for their application. Self assembly in solution on the other hand creates tremendous possibility for the ease of control. By introducing side chains with different types (hydrophilic and hydrophobic) and length, different morphologies such as nanosphere/ball, ${ }^{35}$ nano belts/ribbons, ${ }^{36-39}$ nano-fiber/wire ${ }^{40-44}$ and vesicles ${ }^{45}$ have been generated. Other than solution based self assembly, monolayer formation of selfassembled systems by perylene compounds has also been reported by thermal annealing $45 \mathrm{a}$ and multi layer films by MLBL technique. ${ }^{45 \mathrm{~b} . \mathrm{c}}$ The effect of core group ( perylene unit) extension has also been reported. ${ }^{46}$ Conjugate polymer attached with perylene diimide has been reported to give nano morphologies. ${ }^{35.47-48}$

In the studies from our group ${ }^{45 \mathrm{~d}, 45 \mathrm{e}}$ on the effect of chemical confinement on the self assembly of large aromatic systems, we attached poly (dimethyl siloxane) (PDMS) to either one side of PTCDI or to both sides. In the former case, it is a "macromolecular inverse surfactant" since the head group (aromatic PTCDI) is insoluble. It is also a rodcoil molecule. With PDMS attached to both sides of PTCDI, it is a macromolecular Gemini surfactant, and coil-rod-coil molecule. Vesicles were observed when PDMS was attached to one side of perylene and nano-web morphology by the attachment of PDMS to both sides of perylene. ${ }^{45 \mathrm{~d}_{2} 45 \mathrm{e}}$ In the previous chapter of this thesis, we discussed the rod-coil (macromolecular inverse surfactant) and coil-rod-coil (gemini surfactant) systems in which the coil segment was Jeffamine ${ }^{\circledR}$, a water soluble polymer. The self assembly in water as well as solvent mixtures, the stability of aggregation with 
temperature, and the solid state morphology were discussed. This approach of attaching a polymer into perylene can be considered as a system of chemically (by covalent bonds) confined self assembling chromophore in a polymer matrix. Both PDMS and Jeffamine are highly flexible polymers, the latter being hydrophilic. In these cases, PTCDI is solvophobic, and the polymer segment is soluble in water or a non-aqueous solvent.

In this chapter, we introduce yet another dimension to the self assembly code, as shown in figure 1.3 in Chapter 1. We attach hydrophilic Jeffamine to one side of PTCDI, and an alkyl chain to the other side, resulting in a hydrophilic-solvophobic-hydrophobic system. The interaction between the alkyl chains of adjacent molecules will depend on the van der Waals forces. We expect that the $\pi$ interaction mediated self assembly of perylene would be stabilized by the interaction of the flexible hydrophilic and hydrophobic side chains. In this chapter we discuss the self assembly in aqueous solutions and the drop-cast films. Shorter alkyl amine side chain leads to a folded sheet structure. As we increase the length of the alkyl amine, we observe a ribbon and with further increase in the chain length it forms tube.

\subsection{Experimental}

3, 4, 9, 10-Perylenetrtracarboxylic acid dianhydride, m-cresol and isoquinoline, propyl amine, hexyl amine and octyl amine were purchased from Aldrich Chemical Company Inc. and used as received. Amine terminated polyethylene oxide-propylene oxide copolymer (Jeffamine ${ }^{\circledR}$ ) of average molecular weight 2000 was purchased from Huntsman Inc. and used as received. Chloroform was purchased from Caledon 
Laboratories Ltd. and the other solvents were purchased from Aldrich Chemical Company Inc.

X-ray diffraction (XRD) data were collected using a Philips automated powder diffractometer model PW 1710 and nickel filtered $\mathrm{CuK} \alpha$ radiation $(\lambda=1.542 \AA)$. The MDI Datascan 3.2 software (Materials Data Inc., Livermore, CA) was used for data collection. The results were analyzed with MDI Jade 5.0 XRD pattern processing software. A Zeiss Axioplan 2 imaging universal optical microscope was used to record optical micrographs both in polarized and reflection modes. Either a JEOL JSM-6400 or Tescan Vega-II XMU VPSEM scanning electron microscope was used. The bulk samples were taken on a glass slide or a metal stub. Similarly, a few drops of the solutions in different solvents were deposited on the glass slide or metal stub and then dried at ambient condition for $24 \mathrm{hrs}$, and in vacuum for another $24 \mathrm{hrs}-48 \mathrm{hrs}$. The surface of the samples prepared for SEM was coated with gold (80\%) and palladium (20\%) alloy layer by HUMMER VII Sputtering System (ANATECH Ltd, Alexandria. VA.) to make it conductive before taking the SEM images. The thickness of the conductive layer was 9$10 \mathrm{~nm}$. Some of the images were taken by tilting the sample holder to a maximum of $47^{\circ}$. A TA Instrument 2010 differential scanning calorimeter was used for thermal analysis. The instrument was calibrated with indium reference samples for temperature and energy. DSC traces were recorded with about $7-10 \mathrm{mg}$ of sample in an aluminum pan.

The UV-Vis spectroscopic measurements were performed under ambient conditions using a Varian CARY 3 UV-Vis spectrophotometer. The path length of the 
quartz sample cube was $10 \mathrm{~mm}$. Temperature dependent UV-Vis spectra were taken by Cary 300 Bio UV-Vis spectrophotometer attached with a temperature controller (operating temperature $-10^{\circ} \mathrm{C}$ to $100^{\circ} \mathrm{C}$ ) by Varian. The data was processed by Cary WinUV Software version 3.00 (339). Fluorescence scans were recorded using a Varian CARY Eclipse Fluorescence Spectrophotometer Cary (Varian, Inc., Palo Alto, CA) at $\lambda_{\text {ex }}$ $460 \mathrm{~nm}$ and $417 \mathrm{~nm}$ with a bandwidth of $5 \mathrm{~nm}$ for excitation and $5 \mathrm{~nm}$ for emission. All measurements were performed at room temperature and processed using software CARY Eclipse WinFLR. FTIR spectra of the perylene diimides were taken under ambient conditions by a Varian 1000 Scimitar Series spectrophotometer. The FTIR spectra of the monoimides were recorded using a transparent $\mathrm{KBr}$ pellet with a background correction using the identical sample holder. The waxy red samples of the diimides were dissolved in spectro grade chloroform to make a dilute solution, drops placed on the $\mathrm{NaCl}$ crystal and the FTIR were recorded. The data were processed using the Varian Resolutions (version 4.0.5.009) software. For some of the samples we used ABB BOMEM MB Series spectrophotometer loaded with GRAMS 32AI software.

\subsubsection{Synthesis of Perylene Monoimide Monoanhydride}

In general, Perylene-3, 4, 9, 10-tetracarboxylic diimide with different side chain derivatives (PTCDI) were synthesised by two steps method. In the first step, alkyl amines were attached to the PTDA to get monoimide mono anhydrides. Then the monoimides were converted to diimides of PTDA with asymmetric side chain by reacting with jeffamine ${ }^{\circledR}$. 
In the first phase, monoimide monoanhydrides were prepared by taking appropriate amount of PTDA $(3.81 \mathrm{mmol})$ and alkyl amine $(30.60 \mathrm{mmol})$ solution in water, in a round bottom flask and maintaining the temperature at $60^{\circ} \mathrm{C}$ for 6 hours. For the case of hexylamine and octylamine, water and propanol mixture of different ratio (1:1 and 1:2 respectively) were used due to their poor solubility in water. The reaction mixture was stirred vigorously with magnetic stirrer. After the completion of the reaction the mixture was cooled to room temperature and acidified with $10 \%$ hydrochloric acid, and the precipitate formed was filtered, washed with water to remove amine. The precipitate was dissolved in $1 \%$ hot potassium hydroxide. and filtered to remove insoluble precipitate of diimide. The combined filtrate was heated again to dissolve the precipitate which was formed in cooling. The solution was filtered again to remove the small amount of the floating precipitate of diimide which was passed through the filter. To the filtrate potassium chloride was added until $10 \%$ concentration, and the precipitated potassium salt of monoimide was filtered. The solid was once dissolved in water and then acidified with $10 \%$ hydrochloric acid, and the precipitate formed was filtered, washed with water and dried (yields are $78 \%, 65 \%$ and $64 \%$ for propyl, hexyl and octyl monoimide monoanhydride respectively) to get pure monoimide monoanhydride ( 1 in scheme 4.1$)$. The formation of monoimide was characterized by UV-Vis spectra with concentrated sulphuric acid. The UV spectra of these monoimides in $95 \% \mathrm{H}_{2} \mathrm{SO}_{4}$ gives a characteristic peak with $\lambda \max$ at $581 \mathrm{~nm}$. The IR spectra confirm the synthesis of monoimide monoanhydride by giving the peak at $1771 \mathrm{~cm}^{-1}$ for carbonyl and 1697 and $1657 \mathrm{~cm}^{-1}$ for the imide (Appendix A, figure A5, A6 and A7). 


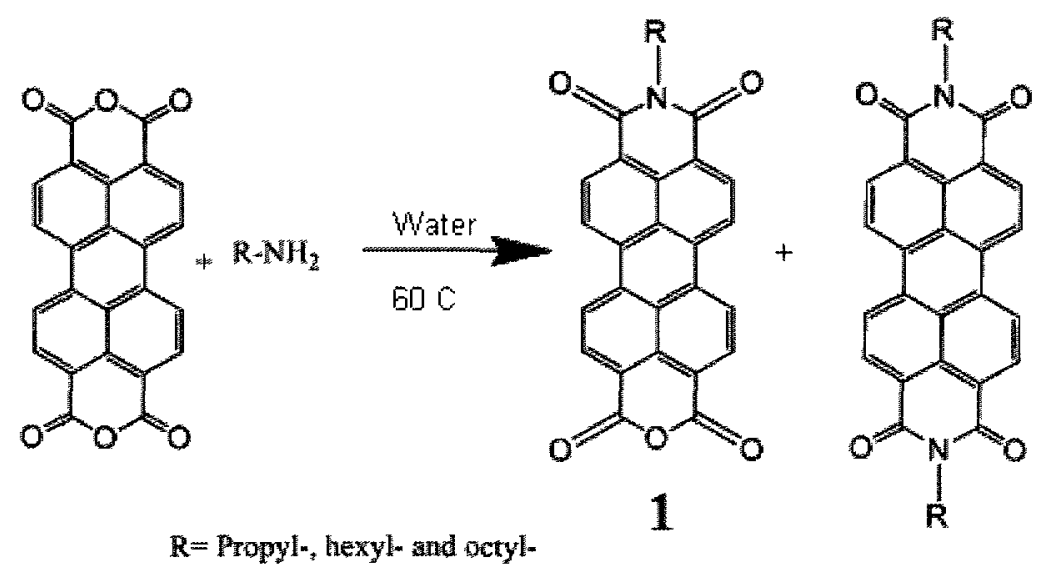

Scheme 4.1: Synthesis of monoimide monoanhydride from PTDA.

\subsubsection{Synthesis of Asymmetric Perylene Diimide}

In a round-bottom flask, appropriate amount of perylene monoimide monoanhydride $(1.10 \mathrm{mmol})$, and Jeffamine $(1.10 \mathrm{mmol})$, and $0.2 \mathrm{~g}$ of isoquinoline were dispersed in $15 \mathrm{~mL}$ of $m$-cresol, and the solution was slowly heated to $200^{\circ} \mathrm{C}$. The reaction mixture was stirred at $200^{\circ} \mathrm{C}$ for $4 \mathrm{~h}$, cooled to room temperature, poured into $300 \mathrm{~mL}$ chloroform. The solution was filtered to remove the unreacted perylene dianhydride if any. The filtrate was collected and it was shaken with acidic water $(\mathrm{pH} \approx$ $5.5)$ in a separating funnel to remove the unreacted jeffamine for several times, if any and the organic layer was separated and the solvent was dried to get very viscous red material (yields are $87 \%, 76 \%$ and $77 \%$ for propyl, hexyl and octyl diimides respectively). The formation of diimide ( 2 in scheme 4.2) was characterized by FTIR and ${ }^{1} \mathrm{H}$ NMR. 


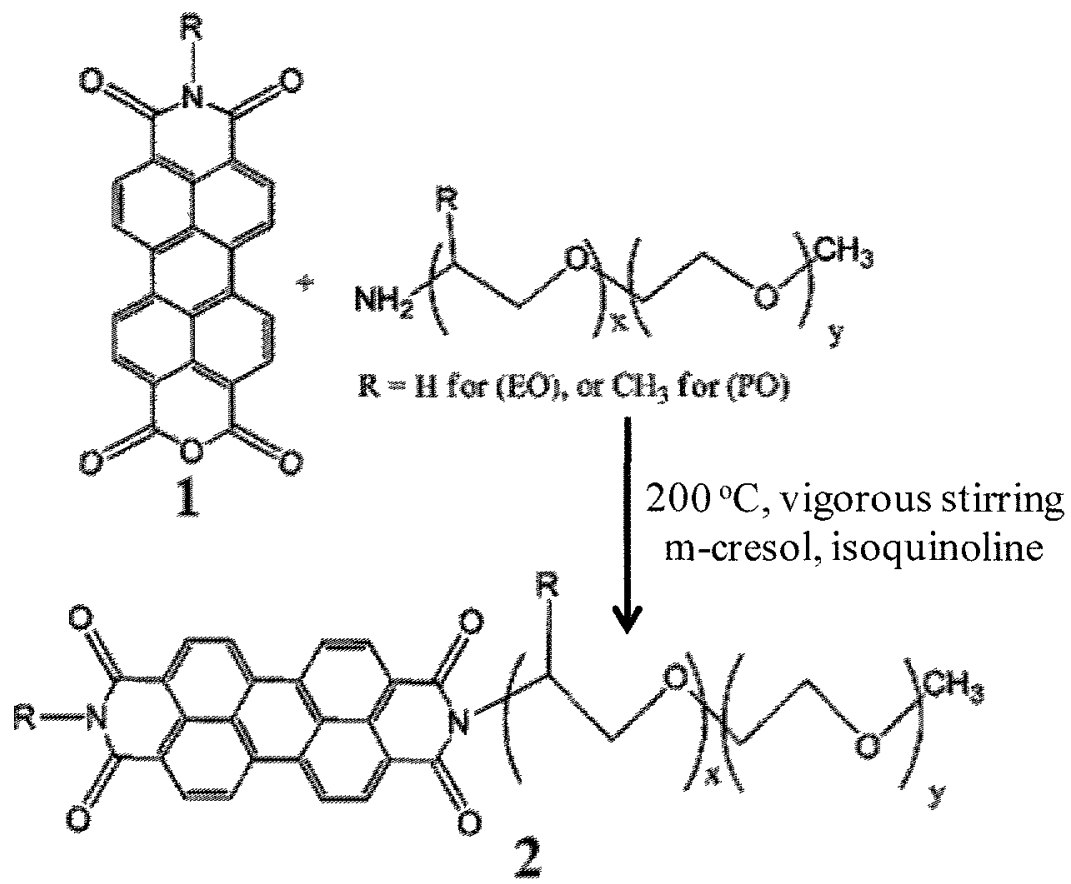

Scheme 4.2: Synthesis of asymmetric perylene diimide.

These diimide samples were dissolved in 95\% sulphuric acid and then UV-Vis spectra was taken. The UV spectra of diimide in $95 \%$ sulphuric acid give a characteristic peak at $598 \mathrm{~nm}$. The FTIR (Appendix A, figure A5, A6 and A7) shows the characteristic peak of imide at 1697 and $1654 \mathrm{~cm}^{-1}$ without any peak at $1700-1800 \mathrm{~cm}^{-1}$. The ${ }^{1} \mathrm{HNMR}$ (Appendix B, figure B4, B5 and B6) gives a multiplet at around 8-8.6 ppm confirms the presence of perylene group.

\subsection{Results and Discussion}

\subsubsection{UV-Vis and Fluorescent Spectroscopy}

The samples with propyl, hexyl and octylamine side chains are denoted by PJPTCDI, HJ-PTCDI and OJ-PTCDI, respectively. The attachment of hydrophilic jeffamine ${ }^{\circledR}$ chain to one side of perylene unit makes it soluble in water and the alkyl 
chain on the other side frustrates its solubility in water due to its hydrophobic nature. The ratios of the molecular weights of hydrophilic to hydrophobic parts in PJPTCDI, HJPTCDI and OJPTCDI are $4.80,4.35$ and 4.10 respectively. Due to the higher molecular weight of hydrophilic Jeffamine. even with increased hydrophobic alkyl side group along the series we observe considerable water solubility of these diimides.

The UV-Vis absorption spectral pattern of all these amphiphilic perylene diimide compounds show the presence of non-aggregated monomeric form in common solvents (acetone, chloroform, dichloromethane. THF, toluene, methanol, TCE etc. ) other than water even with high concentration as seen in figure 4.1. The absorption patterns are almost the same with the $\mathrm{S}_{0-0}$ transition peak with the highest intensity. The absorption spectra in different solvents of different polarity show a small red and blue shift. A red shift in a more polar and a blue shift in a less-polar solvent occur because of different energy levels of the excited states of the perylene moieties. ${ }^{49}$ 

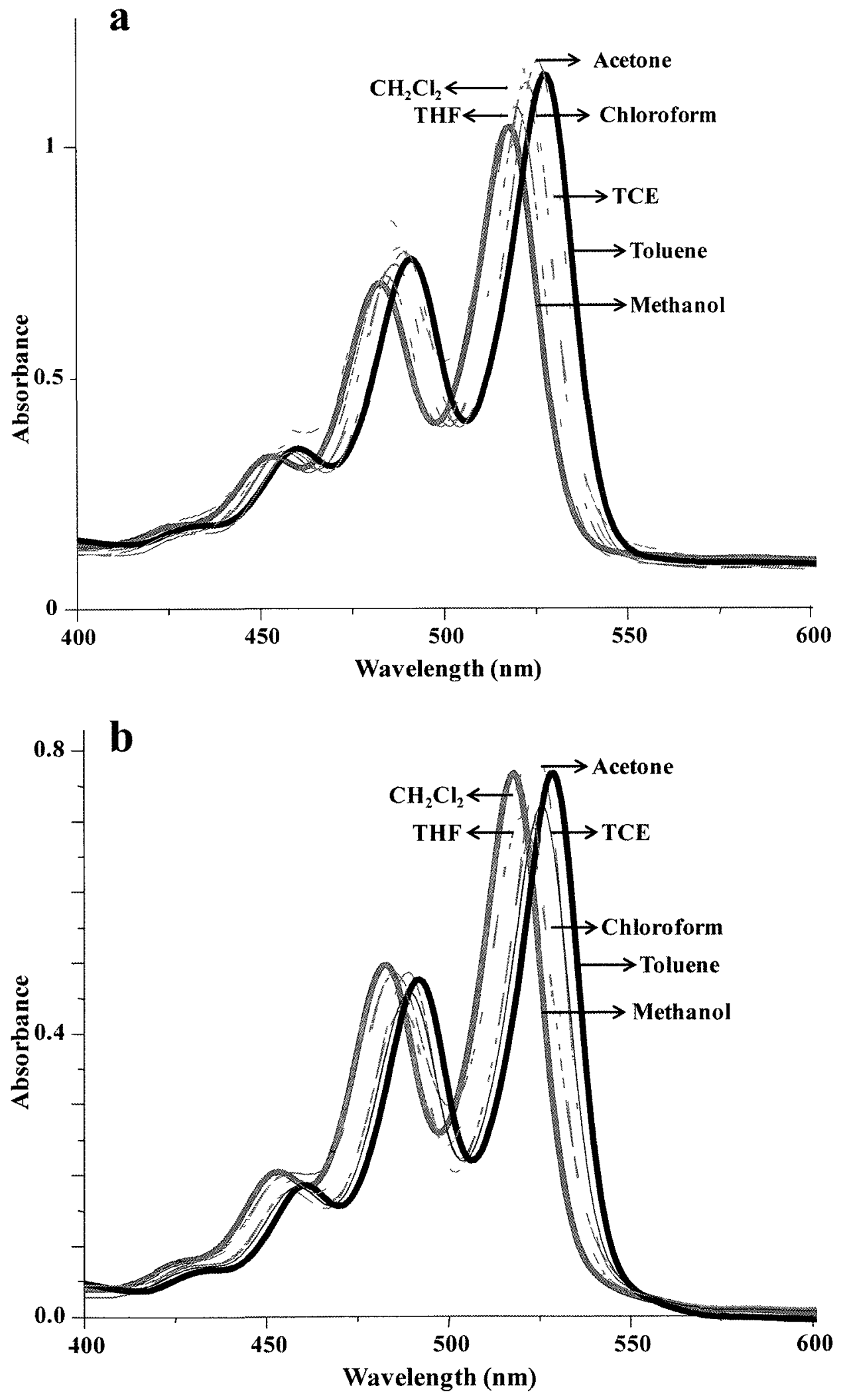

Figure continued in the next page 


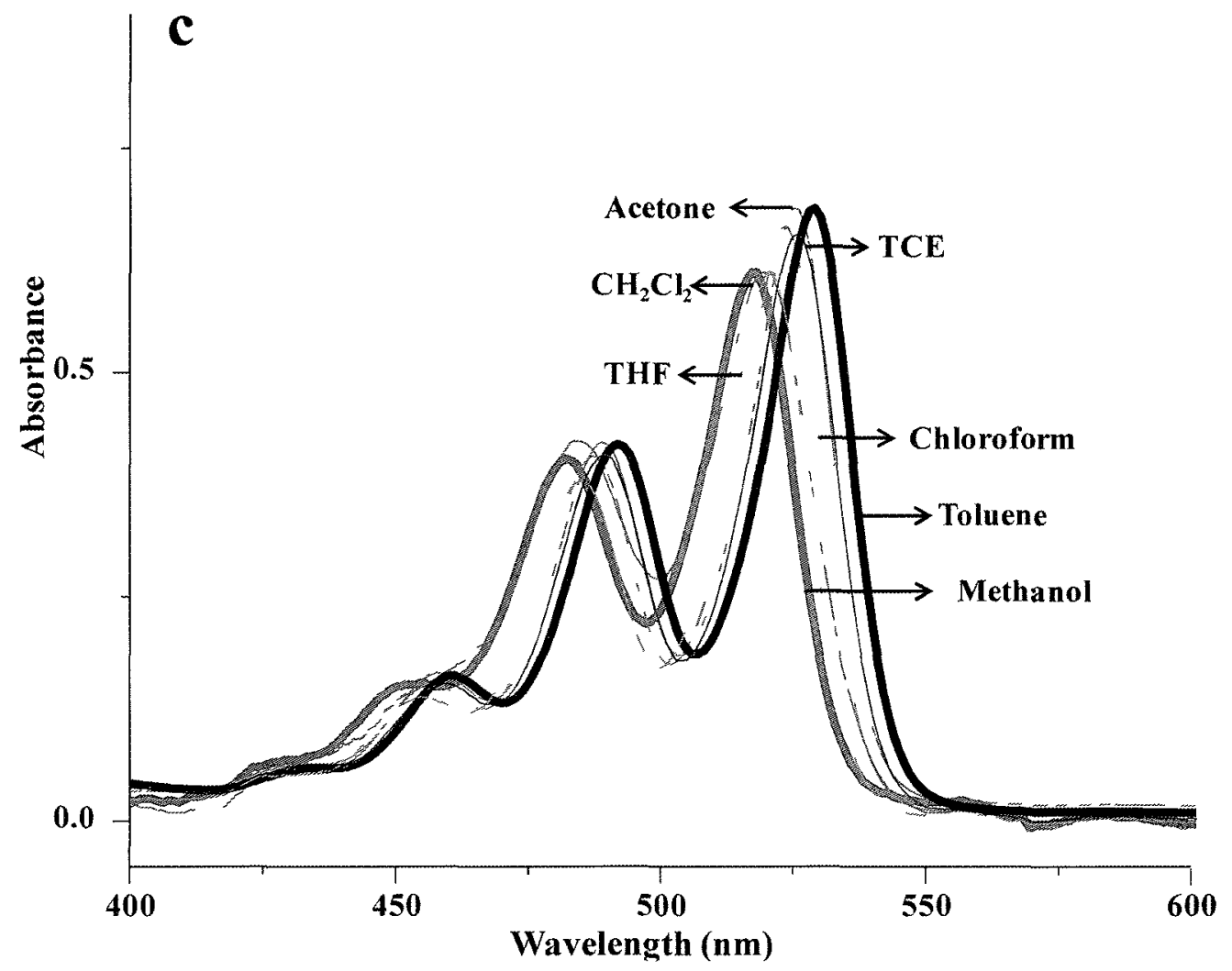

Figure 4.1: The UV-Vis of (a) PJ-PTCDI, (b) HJ-PTCDI and (c) OJ-PTCDI in different solvents at $1 \times 10^{-5} \mathrm{M}$.

The fluorescent emission spectrum shows that with different solvents the pattern is a mirror image of the UV-Vis absorption spectral pattern of PJ-PTCDI with the most intense peak at shorter wave length at all concentrations. For the case of HJ-PTCDI and OJ-PTCDI we observe the same trend of emission peak pattern in different solvents. These diimides show intense fluorescent emission in different solvents after being excited at $366 \mathrm{~nm}$ wavelength (Appendix C. figure C7, C8 and C9). So. the emission color and intensity of the diimides can be tuned by solvents. 
The absorption patterns of these compounds are similar in water at higher concentration $\left(10^{-5} \mathrm{M}\right)$ displaying aggregated pattern of absorption spectra with $\lambda_{\max }$ at lower wavelength. But the absorption pattern of PJ-PTCDI is different at low concentration (below $1 \times 10^{-6} \mathrm{M}$ ) than that of HJ-PTCDI and OJ-PTCDI. In case of PJPTCDI with smaller alkyl chain (propyl in one side) we see aggregated absorption pattern $\left(\lambda_{\max }\right.$ from $\mathrm{S}_{0-1}$ transition) even at very low concentration (below $3 \times 10^{-7} \mathrm{M}$ ). We expected to have a monomeric absorption pattern $\left(\lambda_{\max }\right.$ at higher wavelength) at low concentration. It's a very common trend in short chain substituted perylene diimide to show typical monomeric absorption pattern at low concentration which shows intensity reversal at higher concentration confirming the formation of their aggregate structure. Normally at lower concentration if there is no aggregation typical monomeric absorption pattern is expected where most intense peak will be longer wavelength peak with a very fine vibronic structure in the absorption peak. 

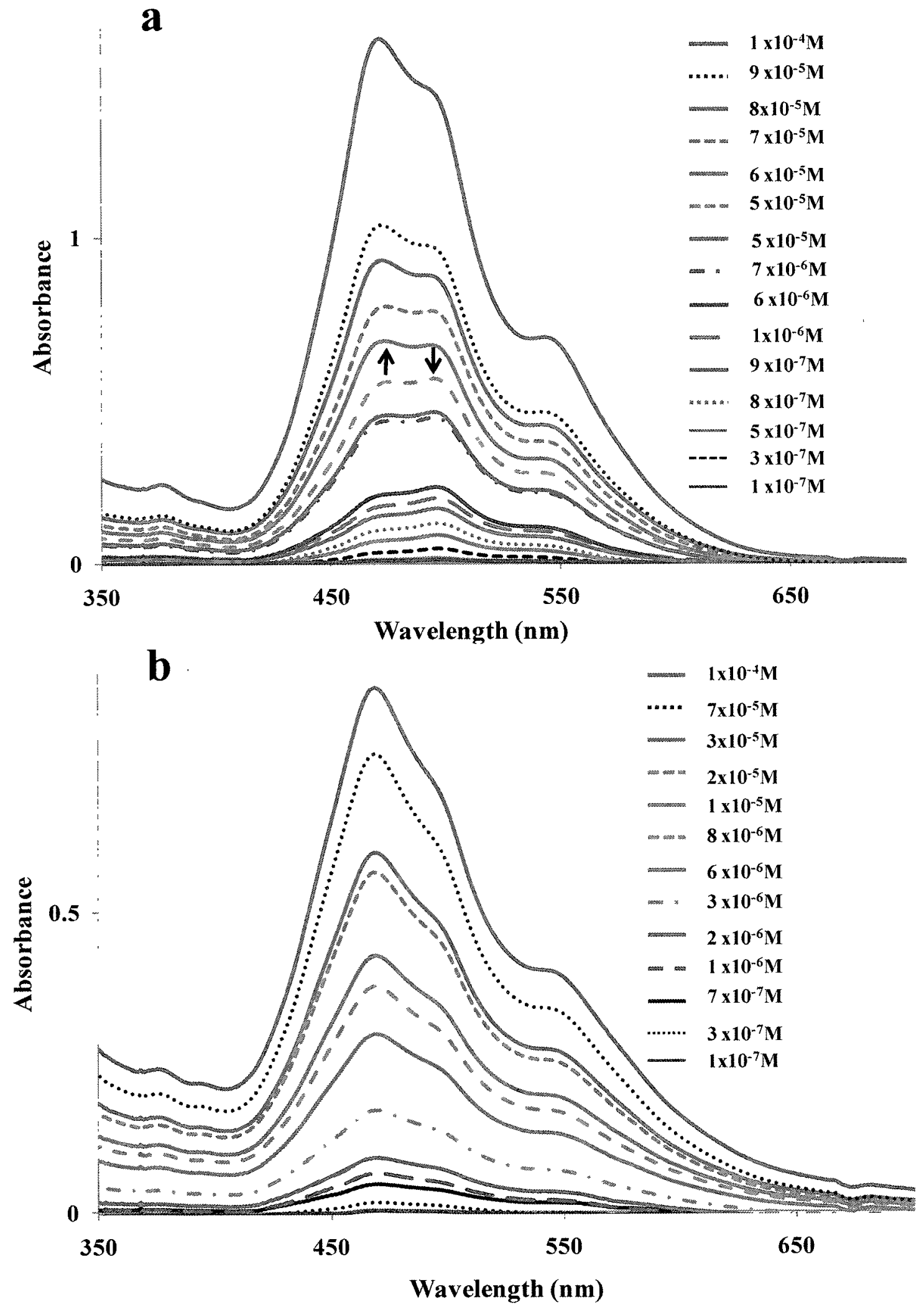

Figure continued in the next page 


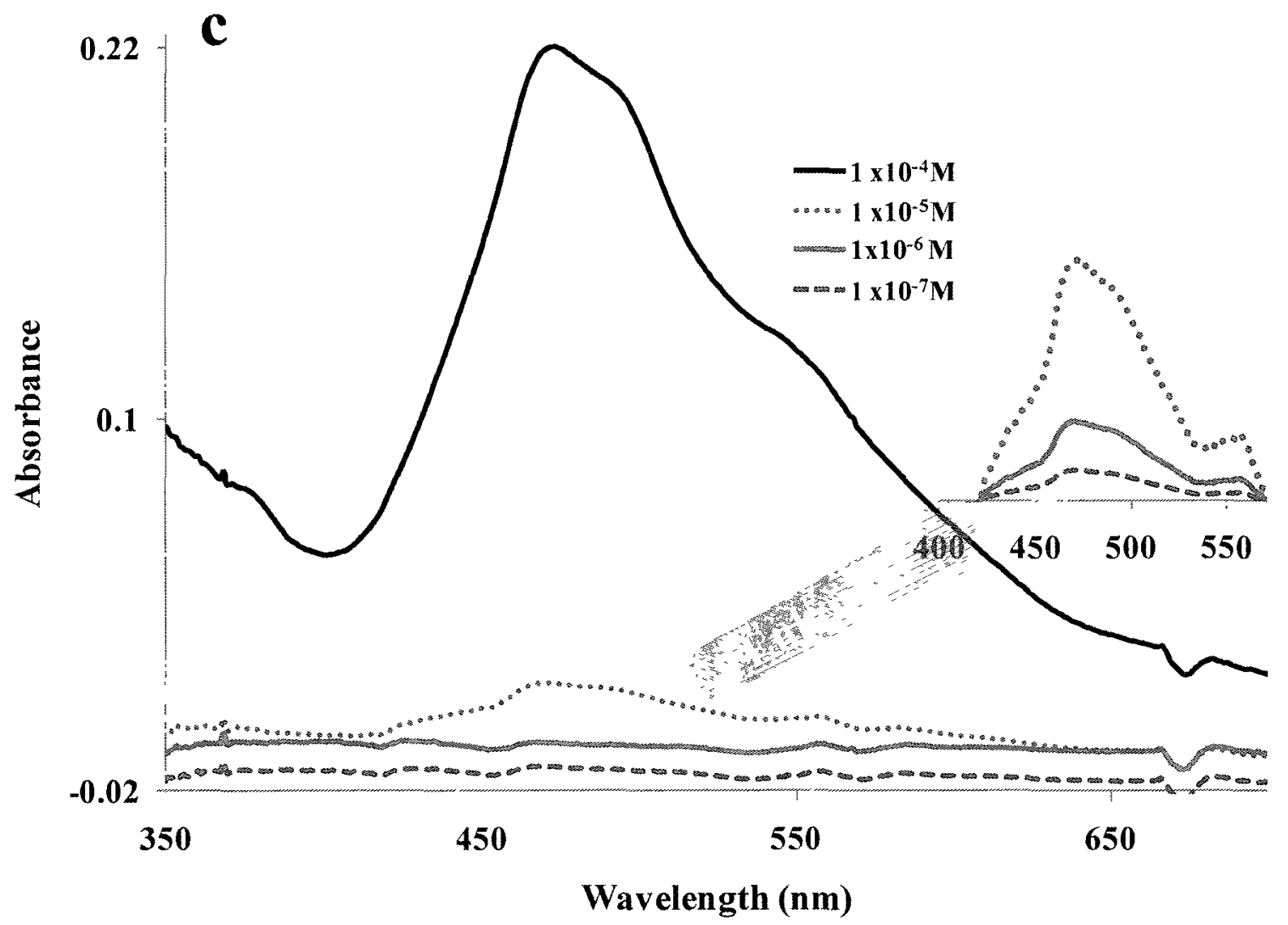

Figure 4.2: The UV-Vis spectra of (a) PJ-PTCDI, (b) HJ-PTCDI and (c) OJ-PTCDI in water from $1 \times 10^{-7}-1 \times 10^{-4} \mathrm{M}$.

Closer look at the absorption pattern for PJ-PTCDI at lower concentration (below $5 \times 10^{-5} \mathrm{M}$ ) reveals that $\mathrm{S}_{0-1}$ peak is intense over $\mathrm{S}_{0-0}$ vibronic transition peak (figure 4.2a) which actually confirms that PJ-PTCDI is in aggregate mode even at very low concentration. Upon increasing the concentration of PJ-PTCDI further, nature of absorption pattern changes and the peak at shorter wavelength $\left(\mathrm{S}_{0-2}\right)$ shows the highest intensity among other transitions. ${ }^{50}$ Thus, below a certain concentration $\mathrm{S}_{0-1}$ transition peak is higher whereas above that concentration $\mathrm{S}_{0-2}$ transition peak becomes higher in intensity. The intensity reversal occurs at $6 \times 10^{-5} \mathrm{M}$ for PJ-PTCDI. A bathochromic shift of absorption peaks is also seen. Besides the intensity reversal, the peaks from $\mathrm{S}_{0-0}, \mathrm{~S}_{0-1}$, 
and $\mathrm{S}_{0-2}$ transitions shift from 545,497 and $473 \mathrm{~nm}$ to 542,492 , and $471 \mathrm{~nm}$, respectively. The presence of $\lambda_{\max }$ at $497 \mathrm{~nm}\left(\mathrm{~S}_{0-1}\right.$ transition $)$ at lower concentration and the appearance of $\lambda_{\max }$ at $473 \mathrm{~nm}\left(\mathrm{~S}_{0-2}\right.$ transition) at higher concentration indicates the presence of two different types of aggregates or the same aggregate with different orientations in it which are switchable with concentration. Figures $4.2 \mathrm{~b}$ and $\mathrm{c}$ show the UV absorption spectra of HJ-PTCDI and OJ-PTCDI, respectively, at various concentrations in water from very dilute to concentrated solutions $\left(10^{-7} \mathrm{M}\right.$ to $\left.10^{-4} \mathrm{M}\right)$. An aggregate structure is evident because of the presence of the most intense $\lambda_{\max }$ at lower wavelength at all concentration. No further change is seen in the absorption pattern with increasing concentrations of $\mathrm{HJ}-$ PTCDI and OJ-PTCDI showing that the same type of stacking exists at all concentrations for HJ-PTCDI and OJ-PTCDI. The solubility of HJ-PTCDI and OJ-PTCDI is comparatively less in water due to greater hydrophobic chain length compared to PJPTCDI.

Normally, an electronic transition is coupled to a single vibration; the intensities of the vibrational transitions within the progression follow the order $\mathrm{S}_{0-0}>\mathrm{S}_{0-1}>\mathrm{S}_{0-2}$ for non-aggregated monomeric form. ${ }^{51,52}$ The phenomenon of intensity reversal (originated from either $\mathrm{S}_{0-1}$ or $\mathrm{S}_{0-2}$ ) in all the cases is associated with aggregate formation. ${ }^{53}$ But the presence of most intense $S_{0-1}$ transition peak at very low concentration and the commencement of intensity reversal from $\mathrm{S}_{0-1}$ to $\mathrm{S}_{0-2}$ at higher concentration for PJPTCDI can be linked to two different modes of aggregation or switching of aggregation mode with concentration. We can rationalize this by the exciton coupling model ${ }^{51,52}$ of aggregate formation which explains the solution and pure-state interactions of organic 
chromophores, assigning aggregate conformations on the basis of the energy of the Frenkel (molecular) absorption. The model describes exciton splitting by the relative dipole interactions of the subunit transitions excluding the aggregate electronic structure. In a planar $\pi$-electron system like the perylene unit, electronic transition is delocalized through the aggregate. Exciton theory predicts that $\mathrm{H}$-aggregation by monomeric perylene unit will lead to a blue shift in the lowest energy allowed electronic transition. Jaggregation is anticipated to only have a blue shift at small distortions from the $\mathrm{H}$ aggregate structure and is typically associated with a red shift that correlates to the extent of displacement (figure 4.3). ${ }^{54-56}$

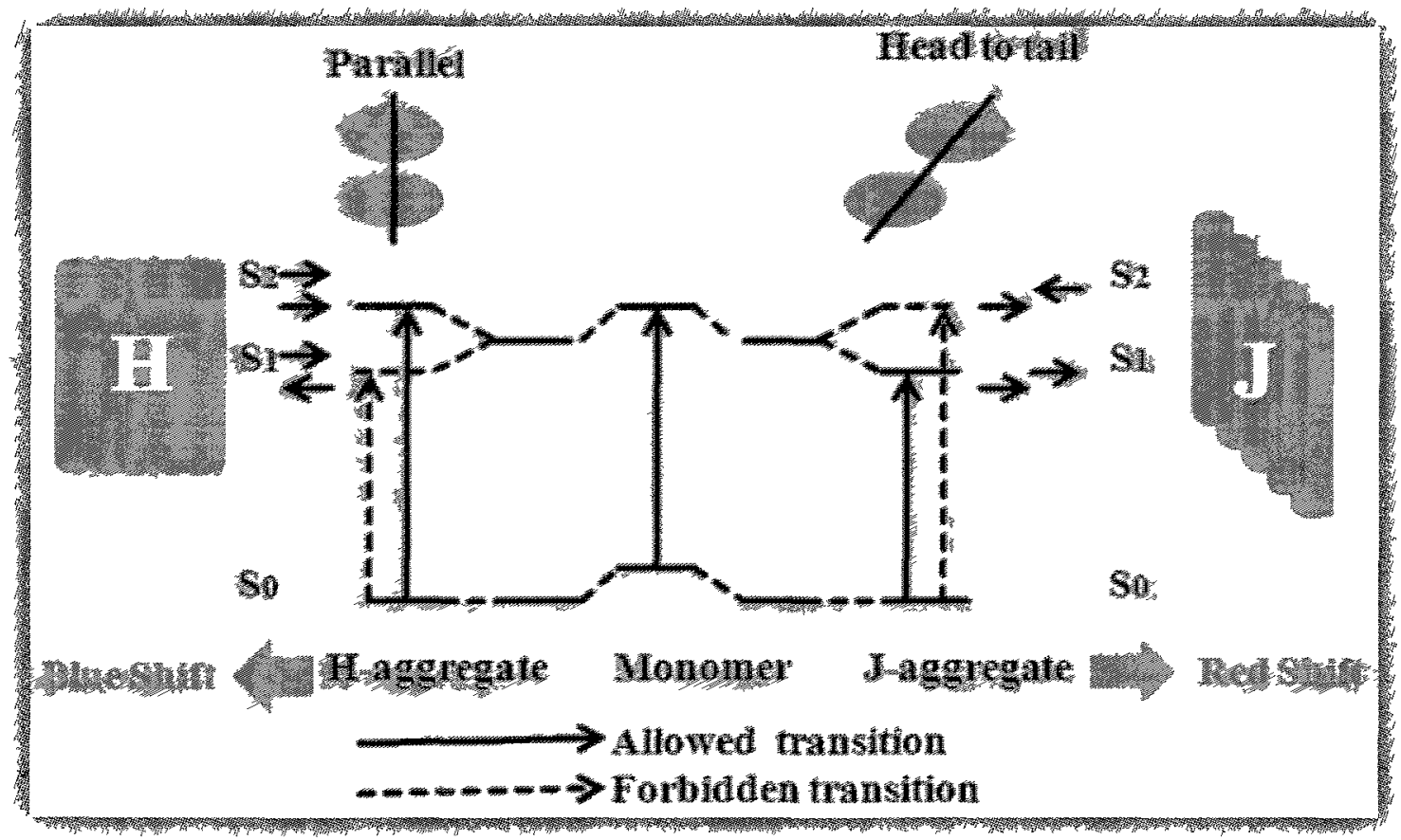

Figure 4.3: $\mathrm{H}$ - and $\mathrm{J}$ - stacking. 


\section{a}
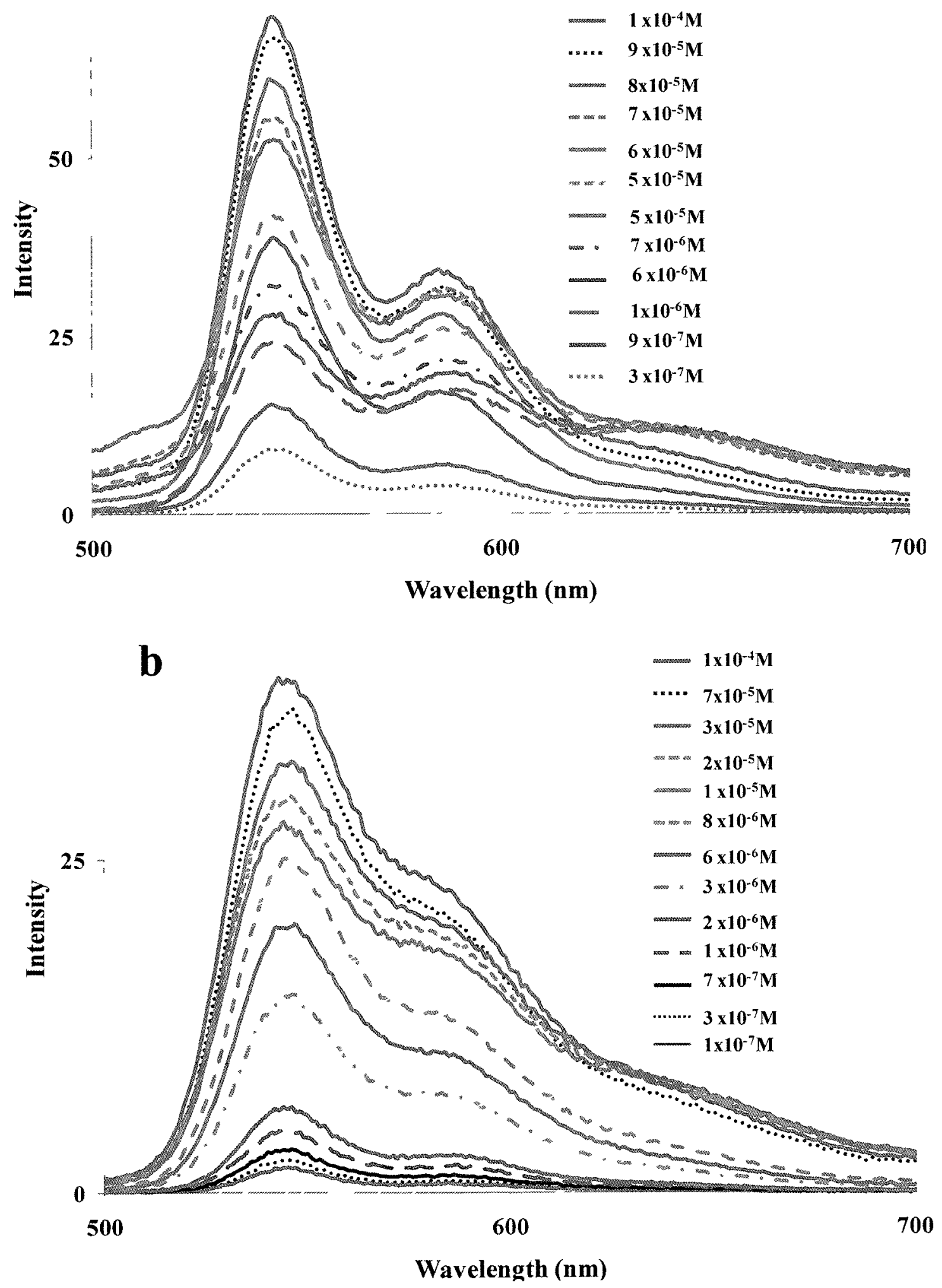

Figure 4.4: Fluorescent spectra of (a) PJPTCDI and (b) HJPTCDI in aqueous solution. 
The small blue shift of all the absorption peaks of PJ-PTCDI with higher concentrations confirms that they are in H-type co-facially arranged aggregates ${ }^{57}$ and the enhanced fluorescent peaks after a certain concentration (figure 4.4a, the emission color of the solutions with different concentration in water is given in Appendix C, figure C10) indicates the formation of J-type aggregates with higher concentration. ${ }^{58-60}$

Thus, the concentration at which $\lambda_{\max }$ moves from $\mathrm{S}_{0-1}$ to $\mathrm{S}_{0-2}$ for PJ-PTCDI is the critical aggregate concentration at which PJ-PTCDI starts switching its mode of aggregation or rearranging to a more favorable aggregate structure whereas at very lower concentration (concentration below $5 \times 10^{-5} \mathrm{M}$ ) it is already in an aggregate structure but in a different or disorganized mode. Another important aspect of the absorption spectra of PJ-PTCDI in water is the broad shapeless absorption pattern at lower concentrations and a sharper absorption pattern at higher concentration which also confirms two different modes of aggregation of PJ-PTCDI in water. ${ }^{56}$ The fluorescent spectra of PJ-PTCDI (figure 4.4a) at different concentrations in water show a sudden quenching at the concentration where intensity reversal occurs confirming the change in aggregation mode from $\mathrm{H}$ - to J-type. Beyond that concentration the intensity of the fluorescent is enhanced. Sudden intensity quenching may be considered as the photo-induced electron transfer process of J-type aggregation.

The aggregation of HJ-PTCDI and OJ-PTCDI occurred at very low concentration $\left(1 \times 10^{-7} \mathrm{M}\right)$. In these cases we don't see any intensity reversal at any concentration from $10^{-4} \mathrm{M}$ to $10^{-7} \mathrm{M}$ other than already present, with $\lambda_{\max }$ at a lower wavelength (figure $4.2 \mathrm{~b}$ and 4.2c). For both HJ-PTCDI and OJ-PTCDI the $\mathrm{S}_{0-1}$ and $\mathrm{S}_{0-2}$ transition peaks at all 
concentration show a single overlapped peak for $\mathrm{S}_{0-2}$ transition with a shoulder for $\mathrm{S}_{0-1}$ transition. This observation also supports the formation of extensive aggregation with higher concentration. So, the aggregation can be controlled by concentration and introducing hydrophilic-hydrophobic side chain on perylene in its imide nitrogen. The fluorescent spectra of HJ-PTCDI shows enhanced intensity (figure 4.4b) with increasing concentration confirming the mode of aggregation as J-type at all concentrations.

With the introduction of the alkyl side chains, three factors influence the self assembly: the hydrophilic Jeffamine, hydrophobic aromatic segment and the hydrophobic alkyl segment. The lengths of the hydrophilic Jeffamine chain and the aromatic segment are the same for all three compounds. The length of the flexible alkyl side chain (in the trans conformation) is $3.84,7.44$ and $10.10 \AA$ for PJ-PTCDI, HJ-PTCDI and OJ-PTCDI respectively whereas the length of the rigid perylene unit is $11.13 \AA$ with a width of 6.69 $\AA$ (figure 4.5). 


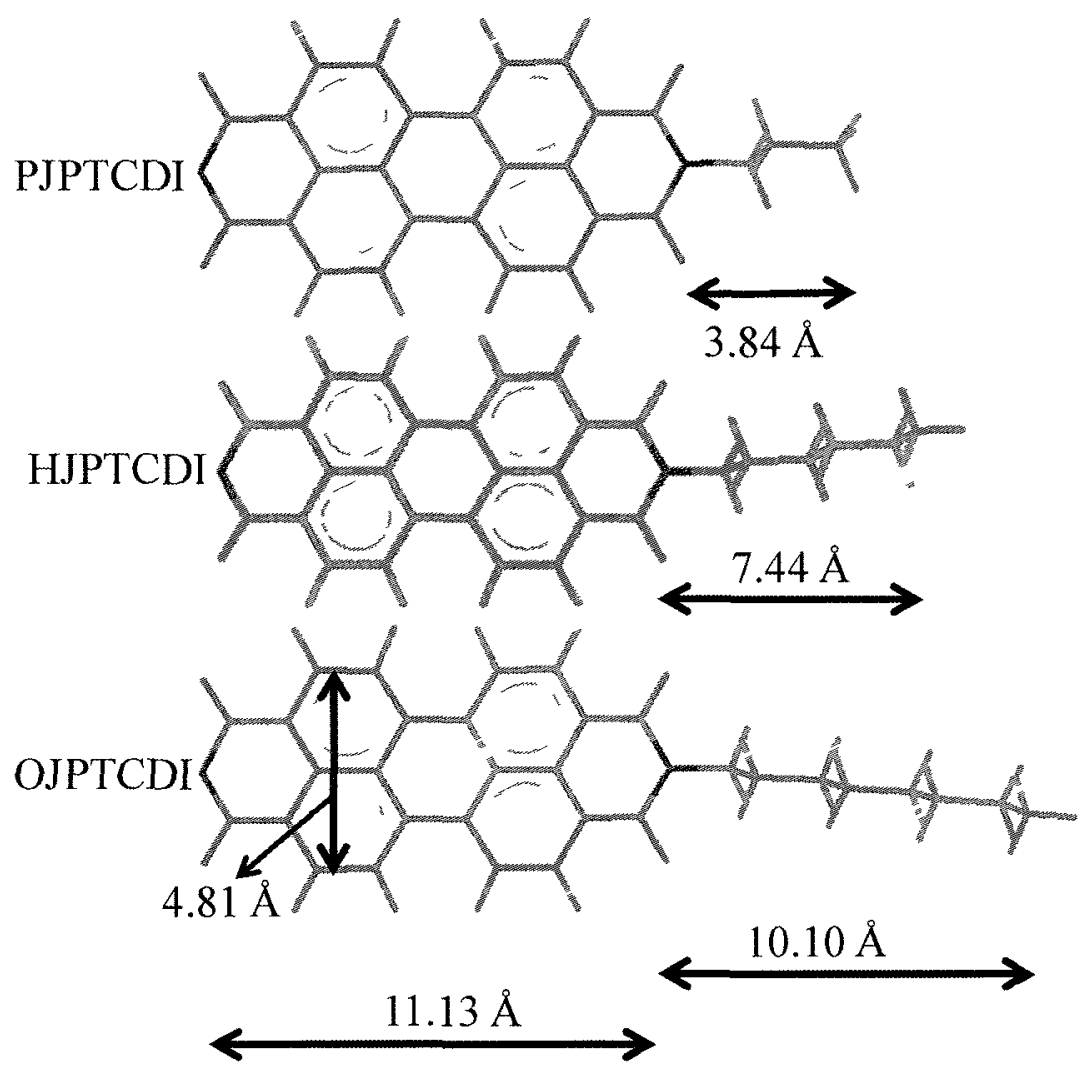

Figure 4.5: Molecular model of the perylene diimides PJ-PTCDI. HJ-PTCDI and OJPTCDI drawn by Hyperchem 7.

The shorter alkyl chain in PJ-PTCDI might lead the molecule to form aggregates at low concentrations giving a rotationally displaced or less ordered H-type aggregate structure, and a rearrangement to J-type mode with an increase in concentration. For the case of HJ-PTCDI and OJ-PTCDI the packing of the longer alkyl chains between themselves would prevent any reorganization.

\subsubsection{Effect of Polarity:}

Stacking of the PTCDI segment occurs with the addition of a non-solvent that cannot dissolve the aromatic part. All these diimides show identical monomeric spectral pattern in nonaqueous solvents (figure $4.1 \mathrm{a}, \mathrm{b}$ and $\mathrm{c}$ ). In more polar solvent (water) these 
diimides prefer to be in the aggregated state. Polarity induces PJ-PTCDI stacking and shifts the $\lambda_{\max }$ towards higher wavelength. Figure 4.6 shows the absorption and emission spectra of PJ-PTCDI in acetone/water mixtures. In this case (water-acetone and watermethanol) intensity reversal occurs at $6 \times 10^{-5} \mathrm{M}$, the absorption spectrum shows a gradual shift of absorption maxima as well with addition of water. With acetone/water: $30 / 70 \%$ the $\mathrm{S}_{0-1}$ transition becomes more intense then the $\mathrm{S}_{0-0}$ transition. A bathochromic shift of $\lambda_{\max }$ occurs upon increasing the polarity and the well structured absorption pattern changes to a less structured peak (The emission color from excitation at $366 \mathrm{~nm}$ of the solution with different water $\%$ is shown in Appendix C, figure C11). This bathochromic shift with intensity reversal confirms that with increasing water content hydrophilic jeffamine becomes more and more extended which helps maximize the $\pi$ - $\pi$ stacking. 

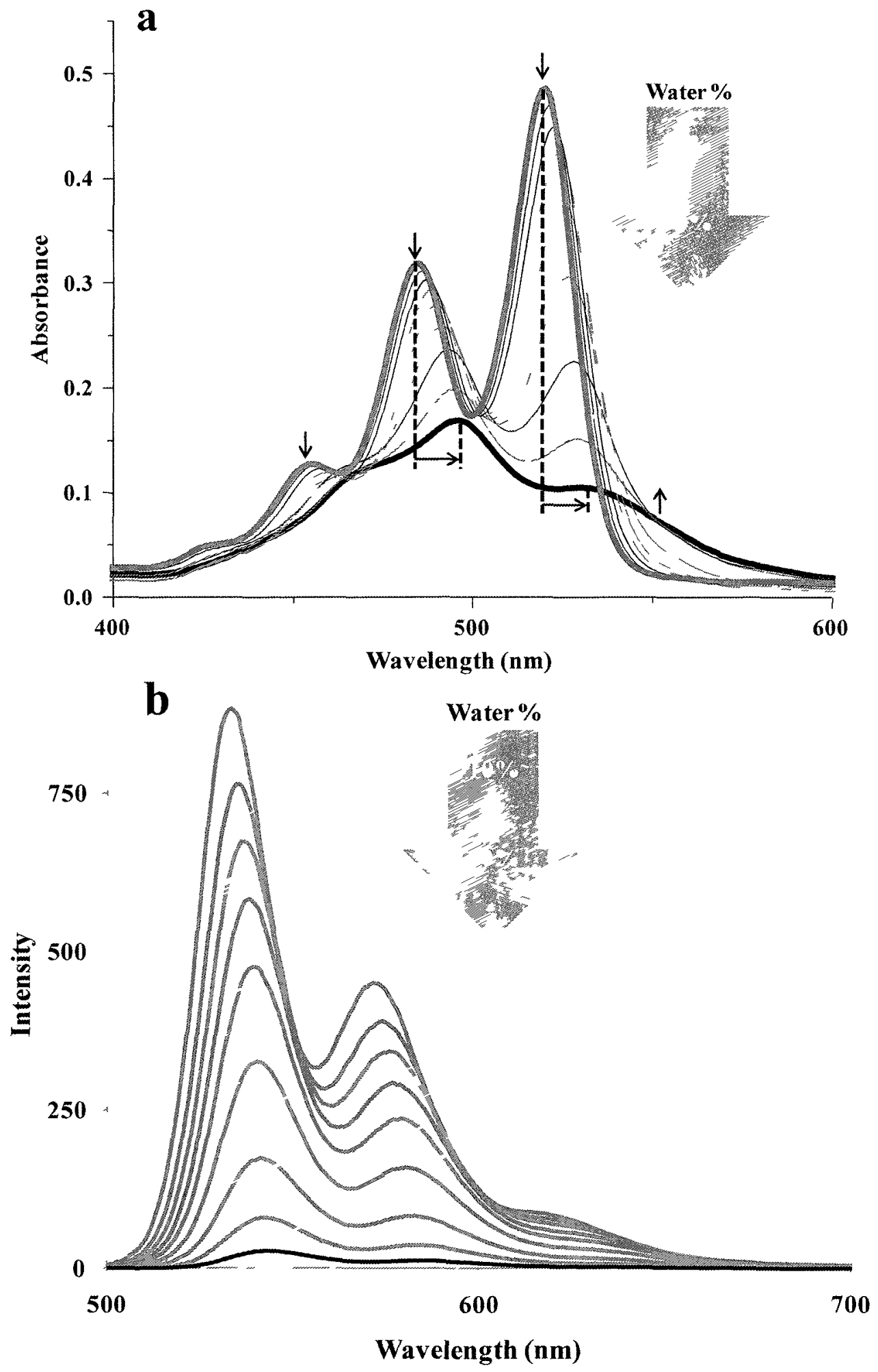

Figure 4.6: (a) UV-Vis and (b) Fluorescent spectra of PJ-PTCDI in acetone-water mixture at $1 \times 10^{-5} \mathrm{M}$. 

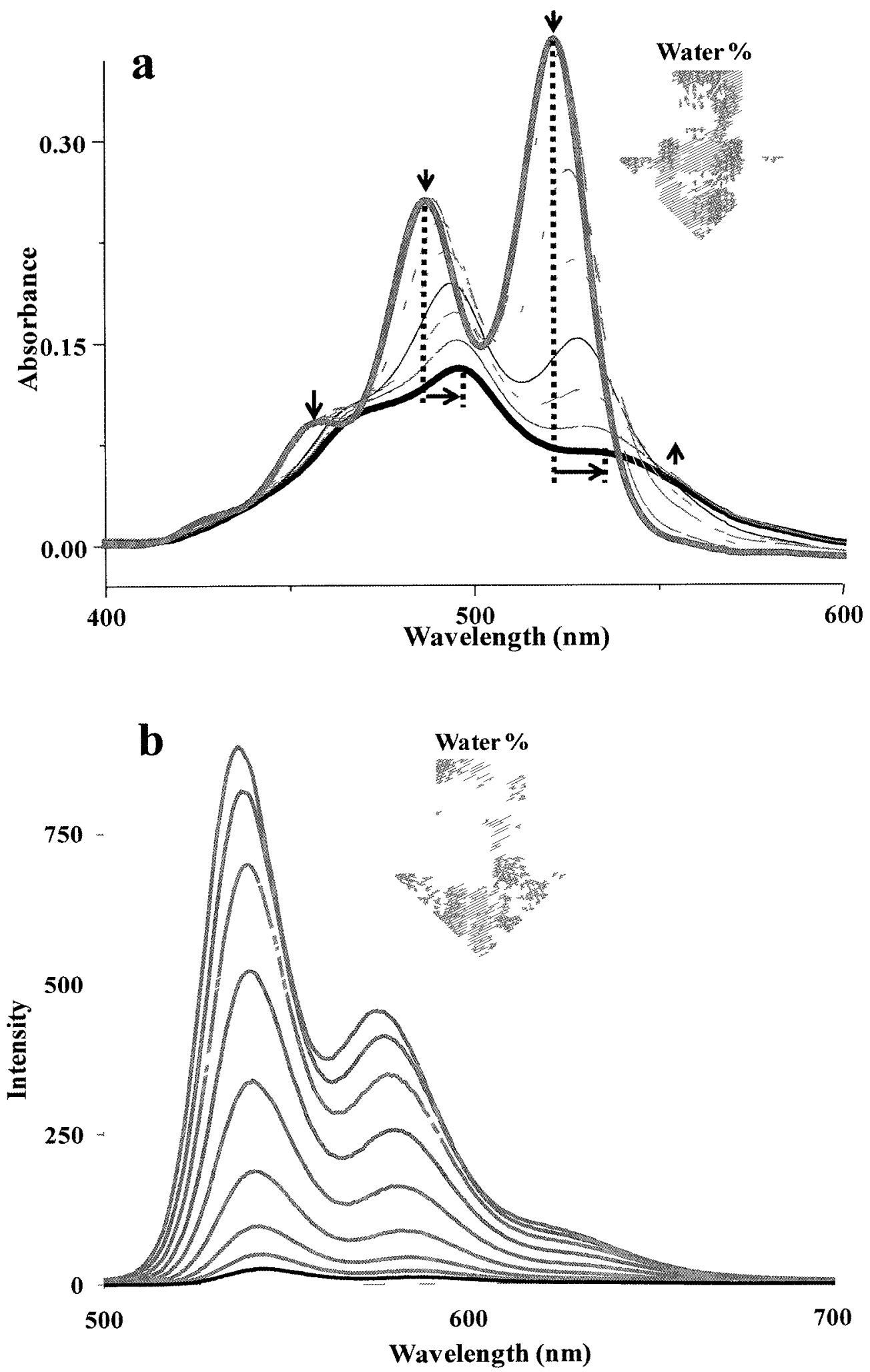

Figure 4.7: (a) UV-Vis and (b) fluorescent spectra of PJ-PTCDI in methanol-water mixture at $1 \times 10^{-5} \mathrm{M}$. 
The same trend is seen with methanol/water mixtures (figure 4.7 ), but the intensity reversal occurs at $50 \%$ methanol content (figure $4.7 \mathrm{a}$ ) (The emission color from excitation at $366 \mathrm{~nm}$ of the solution with different water \% is shown in Appendix C, figure C12). The fluorescent spectra of PJ-PTCDI in acetone-water (figure 6b) and methanol-water mixtures (figure $4.7 \mathrm{~b}$ ) show that with the increase of water $\%$ the aggregation starts forming with a reduction in fluorescent intensity. The reduction of fluorescence intensity with the aggregation is due to the formation of strong $\pi-\pi$ interaction mediated aggregates and the quenching at higher concentration can be explained as photo-induced electron transfer within the aggregates of higher order. ${ }^{12}$ This indicates that in the case of perylene attached to long hydrophilic polymer and short hydrophobic group the solvent polarity can be used significantly to initiate the controlled self assembly of perylene chromophores.

\subsubsection{Effect of Temperature:}

Temperature dependent UV -Vis spectra of PJ-PTCDI were recorded from 20 to $90{ }^{\circ} \mathrm{C}$ (figure $\left.4.8 \mathrm{a}\right)$. With an increase in temperature the most intense peak ( $\lambda_{\max }$ at 494$)$ from $S_{0-1}$ transition becomes less intense and shoulder-like and the $S_{0-2}$ transition peak $\left(\lambda_{\max }\right.$ at 456$)$ becomes dominant confirming the slow breakdown of the aggregate structure. The absorption spectra of PJ-PTCDI were also recorded in acetone/ water (1/9) and methanol/water (1:9) mixtures 10 to $80{ }^{\circ} \mathrm{C}$ (figure $4.8 \mathrm{~b}$ and $4.8 \mathrm{c}$ ). A small hypsochromic shift of $\lambda_{\max }$ is seen along with the intensity reversal between and $S_{0-1}$ and $\mathrm{S}_{0-0}$ transition with temperature. The hypsochromic shift of $\mathrm{S}_{0-1}$ and hyperchromic shift of $\mathrm{S}_{0-0}$ transition peaks confirm the slow dissociation of aggregated structure to monomeric species. In the case of HJ-PTCDI (figure 4.8d) the aggregated structure doesn't 
breakdown, keeping the same pattern of absorption peak with reduced intensity (hypochromic shift) with slightly hypsochromic shift with temperature in water.
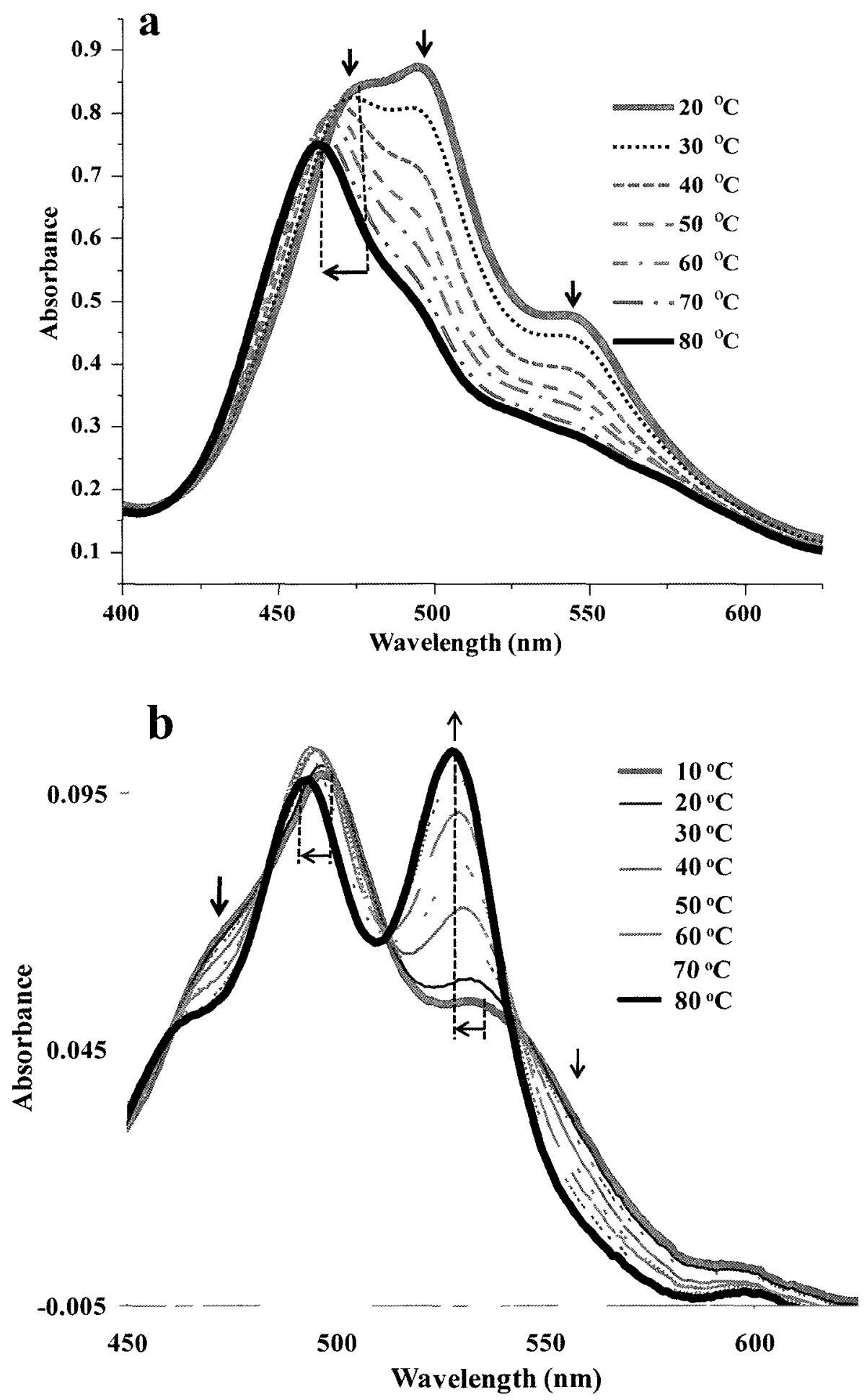

Figures continued in the next page 


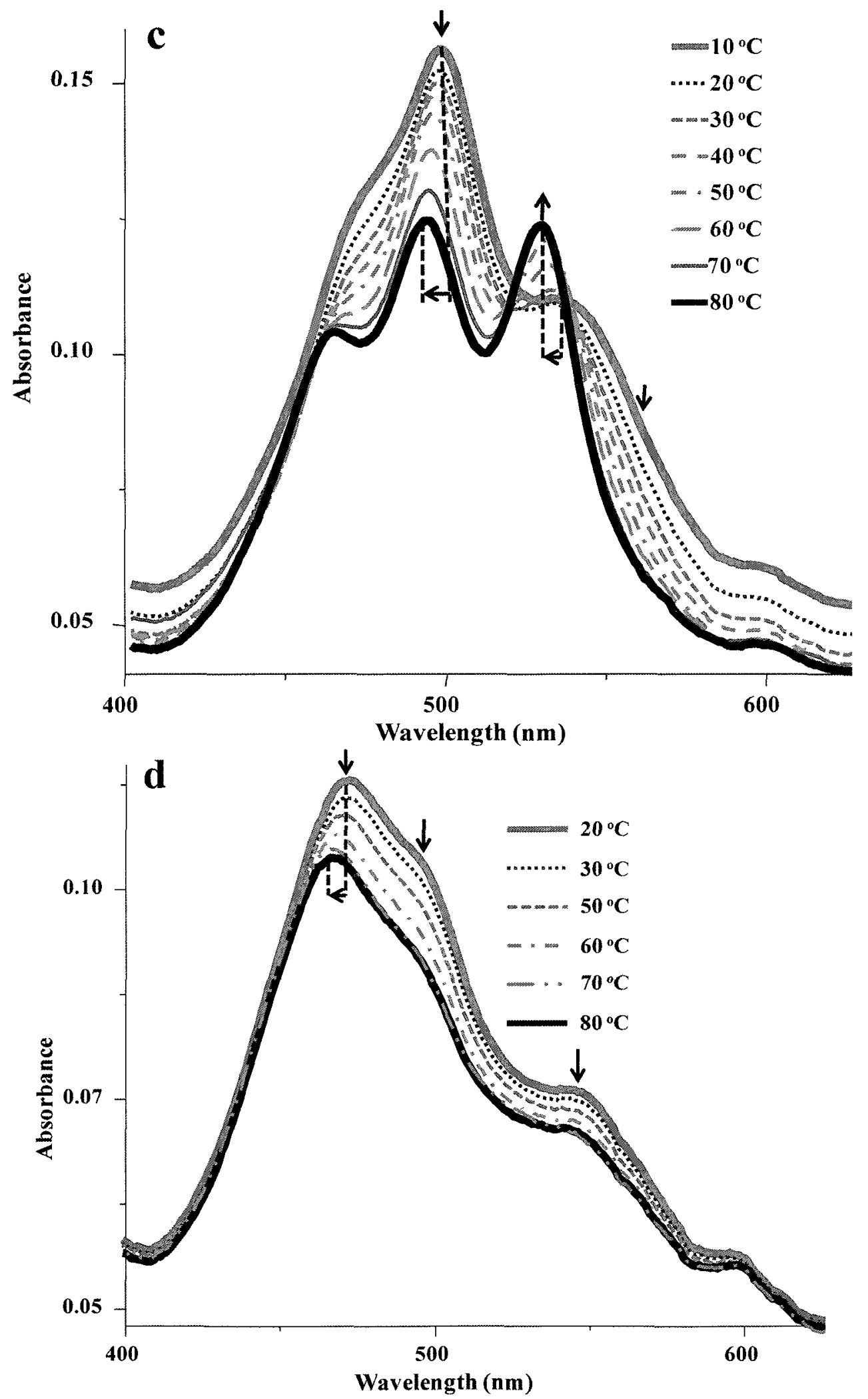

Figure 4.8: Temperature dependent UV-Vis spectra of PJ-PTCDI in (a) water, (b) in acetone-water (c) in methanol-water and (d) HJ-PTCDI in water at $10^{-5} \mathrm{M}$. 


\subsubsection{Microscopy:}

The optical micrographs of the as-synthesized bulk samples show that perylene diimide aggregates are needle-like for PJ-PTCDI (figure 4.9a). The frequency and the density of the crystals (average dimension per 10 measurements in 10 different areas) are greater in case of PJ-PTCDI. As we increase the length of the hydrophobic side chain length the crystals become more ribbon like and the width of the ribbons are also increased from $25 \mu \mathrm{m}$ for PJ-PTCDI to a few hundred $\mu \mathrm{m}$ for OJ-PTCDI (figure $4.9 \mathrm{~b}$ and $4.9 c)$.

From this observation we can say that the greater the side chains of the hydrophobic chain length the greater $\pi-\pi$ interaction among the perylene units and hence the larger the aggregate size. This happens due to the greater extension of the side chain towards the exterior of the perylene moiety. 

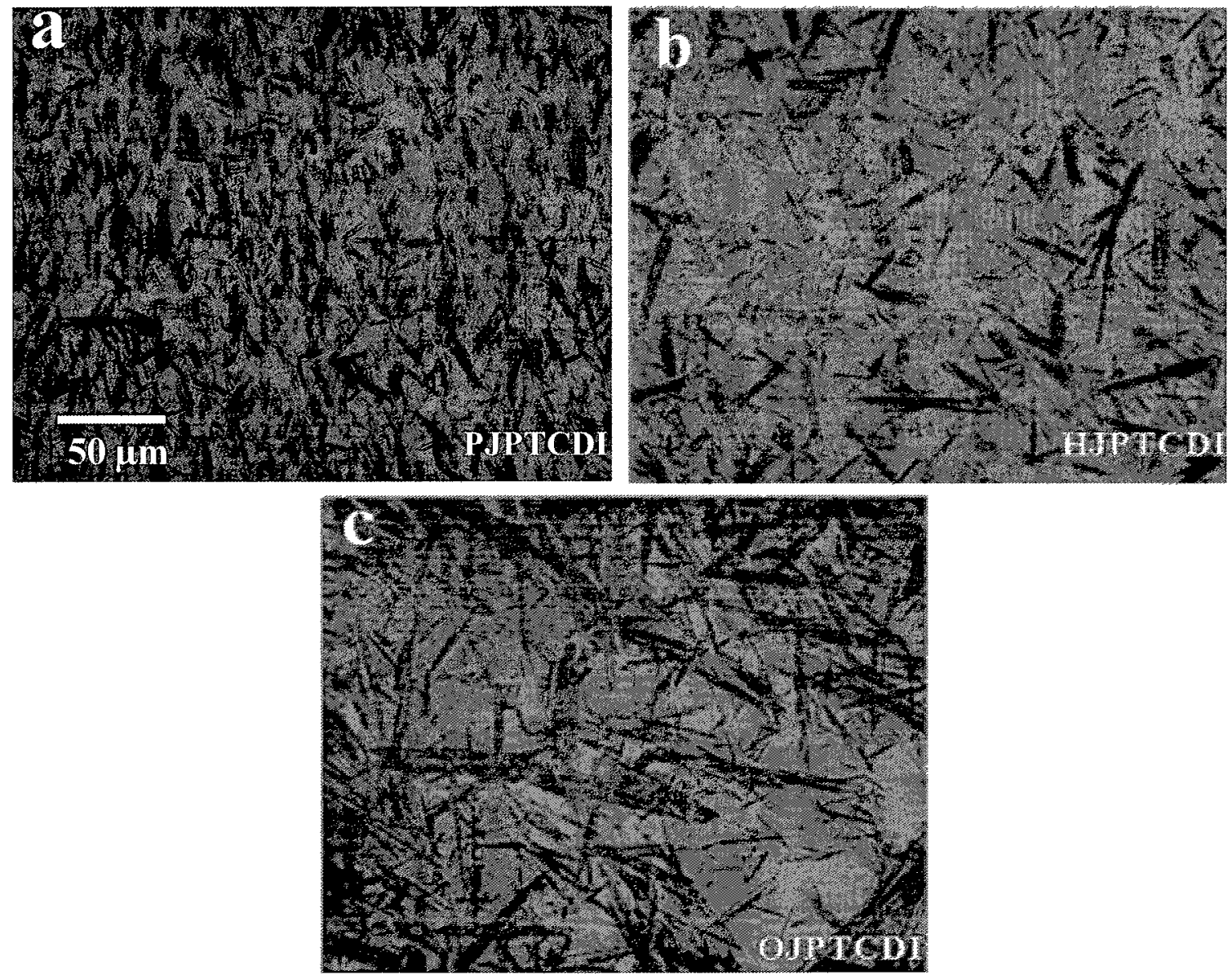

Figure 4.9: The optical micrographs of pure (a) PJ-PTCDI, (b) HJ-PTCDI and (c) OJPTCDI. The scale bar is same for all. 

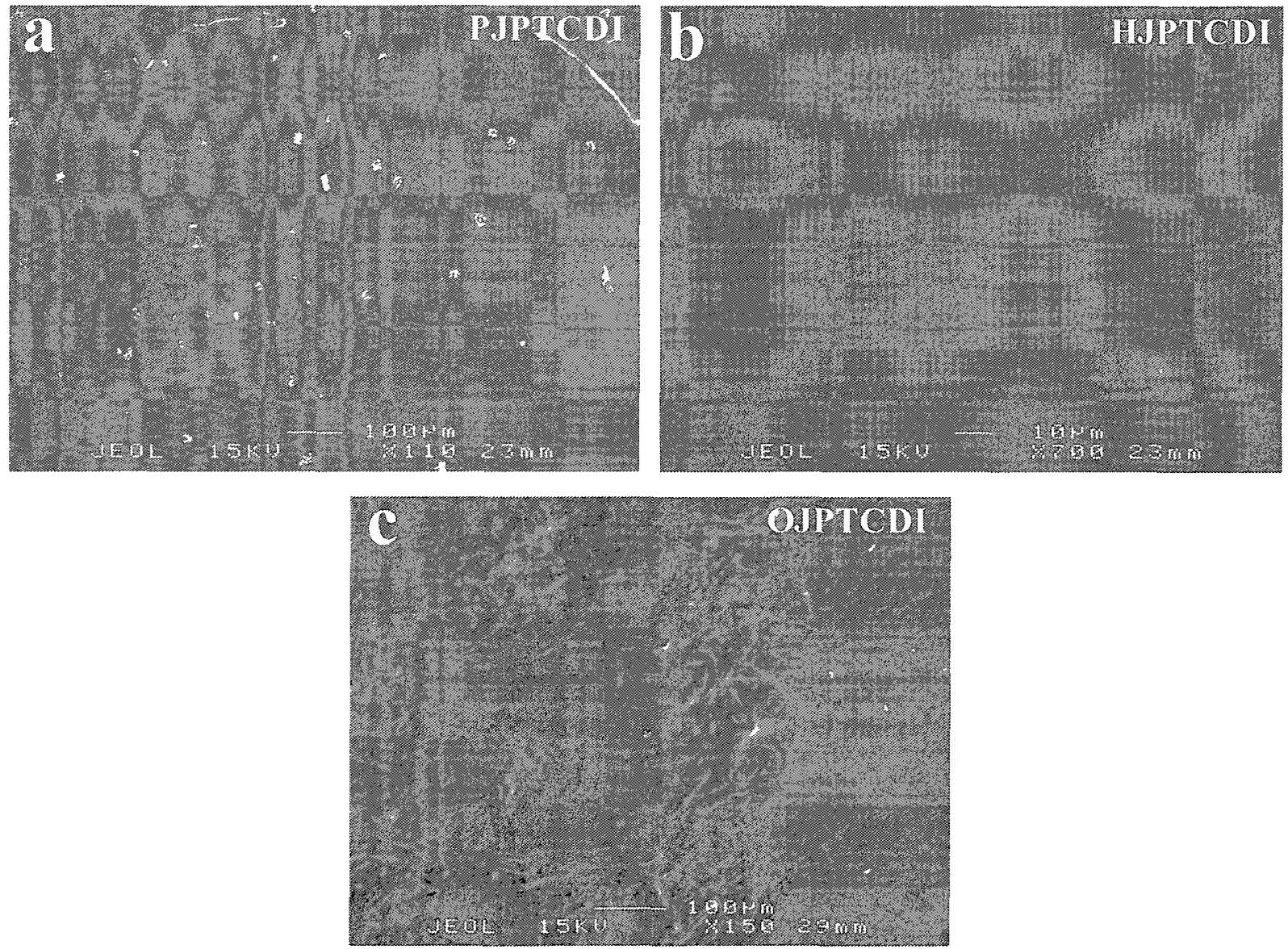

Figure 4.10: The SEM of as such (a) PJ-PTCDI. (b) HJ-PTCDI and (c) OJ-PTCDI.

The SEM (figure 4.10) of the as-synthesized samples also shows such aggregate structures. However, as the SEM images only the surface only the clarity is poor due to the Jeffamine coating the aggregates. We reported a difference in the self-assembled 
structures in the solution phase of these diimides if they are deposited from aqueous solution.

The above noted aggregate structures become more visible in the SEM images of drop cast films (from $10^{-4} \mathrm{M}$ aqueous solutions). The morphology of the aggregates changes from small bundles to ribbons (figure 4.11) with an increase in the hydrophobic chain length. It appears that longer alkyl chains lead to better packing of the perylene unit. Whereas with a short alkyl chain on one side for PJ-PTCDI, the perylene packing leads to small folded sheet aggregate crystals (figure 4.11a), the longer alkyl chains facilitate the development of planar ribbon like aggregates (figure $4.11 \mathrm{~b}$ ) for the case of HJ-PTCDI. With further increase in the chain length in case of OJ-PTCDI, it starts extending and more effective overlap gives both ribbon and tubular structures (figure 4.11c). We have shown before that when these self-assembled structures form sheets, such flat sheets are unstable and wrap into eaves trough or tubular structures. ${ }^{61}$ From the tilted SEM $\left(45^{\circ}\right)$ the hollow nature of the tube is clearly seen (figure $4.11 \mathrm{~d}$, e and f) which seems to be the bundle of many sheets (figure $4.11 \mathrm{~d}$, e and $\mathrm{f}$ ). The sheet (Figure 4.11f with mark 1) and the tube (figure $4.11 \mathrm{f}$ with mark 2) are marked in the SEM image of OJ-PTCDI. 

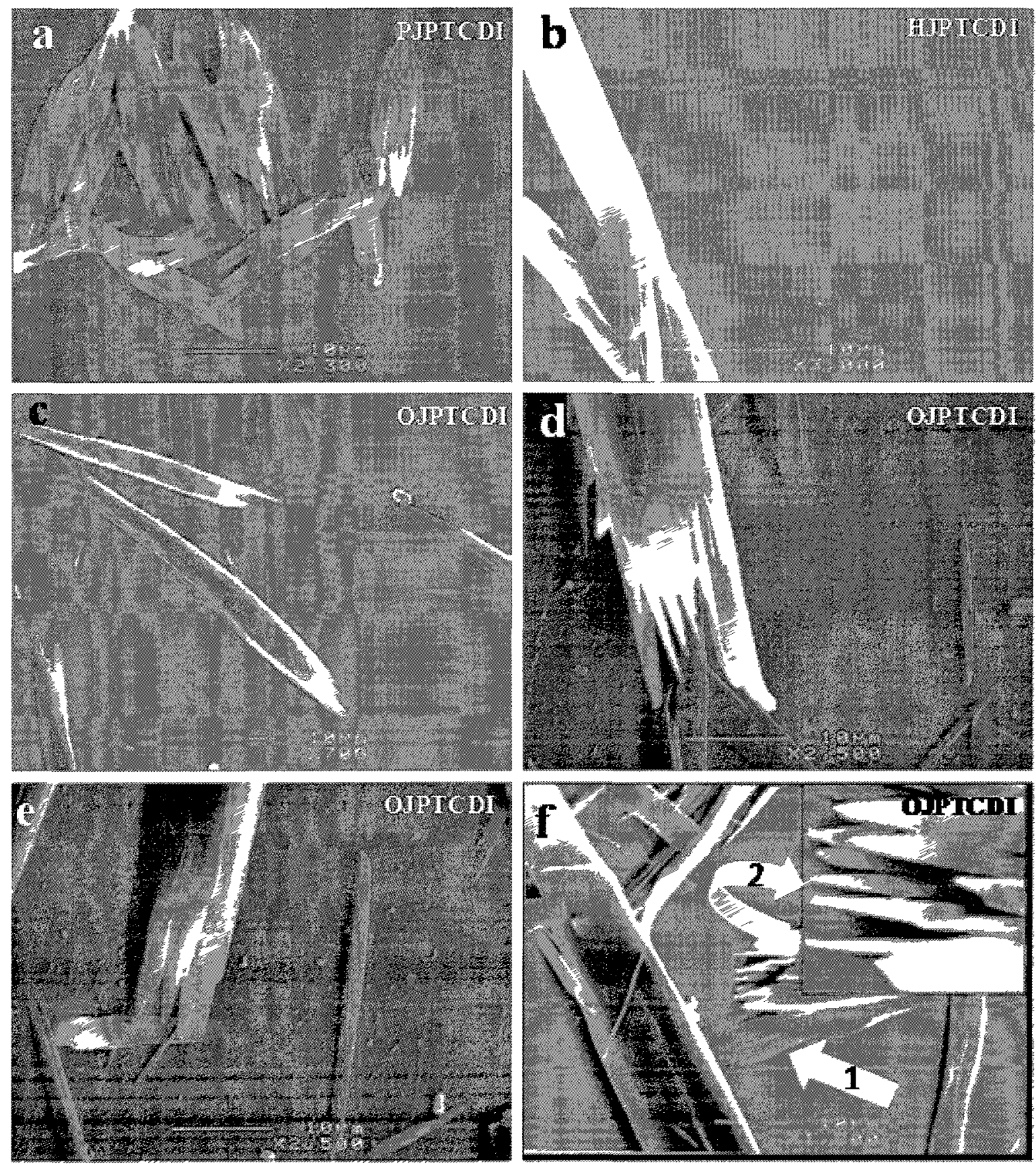

Figure 4.11: The SEM of drop cast samples of (a) PJ-PTCDI, (b) HJ-PTCDI and (c) OJPTCDI respectively in aqueous solution. The samples were prepared by dropping the solutions on the SEM glass slides and then dried by ambient condition Here $\mathrm{d}$, e and $\mathrm{f}$ images were taken by tilting the slide of the sample by $47^{\circ}$ to see the tube nature of the packing from OJ-PTCDI. 


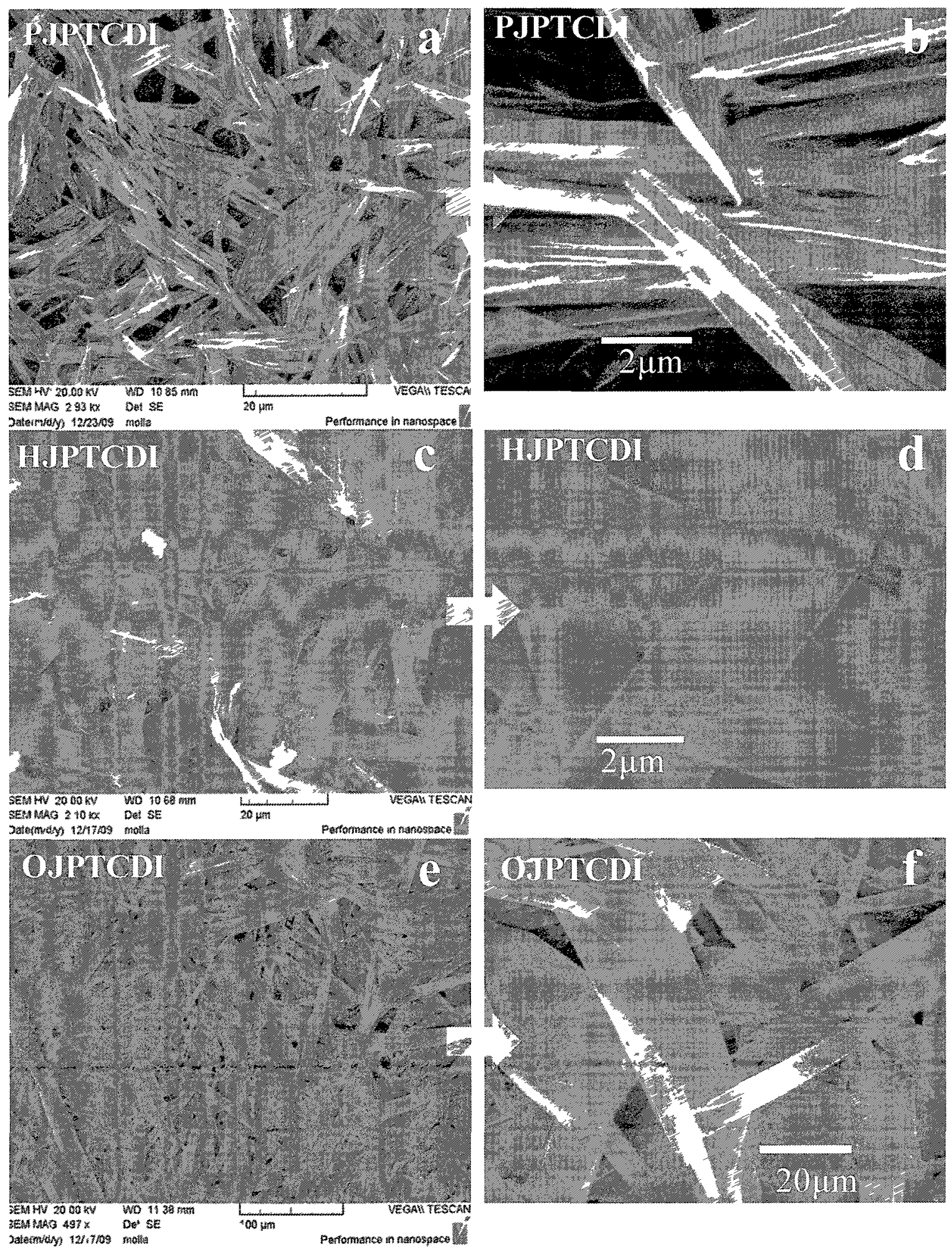

Figure 4.12: SEM images of slowly formed self assembled structures of different diimides from $10^{-5} \mathrm{M}$ aqueous solution. 
Figure 4.12 shows the SEM images of samples derived from slow self assembly

from $10^{-5} \mathrm{M}$ water solutions, which were pipette, drop cast on glass slides and then dried. They were dried at ambient temperature for $24 \mathrm{hrs}$ and another $24 \mathrm{hrs}$ in vacuum. These are similar to those shown in figure 4.11, with folded bundle of sheets for PJ-PTCDI (figure $4.12 \mathrm{a}, \mathrm{b}$ ), very transparent sheets from HJ-PTCDI (figure $4.12 \mathrm{c}, \mathrm{d}$ ) and sheet and tube from OJPTCDI (figure $4.12 \mathrm{e}, \mathrm{f}$ ). Although these diimides did not show aggregation in non-aqueous solutions, they form aggregate structures upon drying $\left(10^{-5} \mathrm{M}\right.$ solutions). Figure $4.13 \mathrm{a}$ shows the same folded sheet morphology for PJ-PTCDI in acetone, chloroform and methanol and smaller fibrous morphology in THF. HJ-PTCDI shows spherical morphology in chloroform and pile of needle in THF (figure 4.13b) where as OJ-PTCDI forms extensive network of fibers in chloroform and pile of needles in THF (figure $4.13 \mathrm{c}$ ). In other solvents all of them preserve their morphology similar to water. 


\section{a}
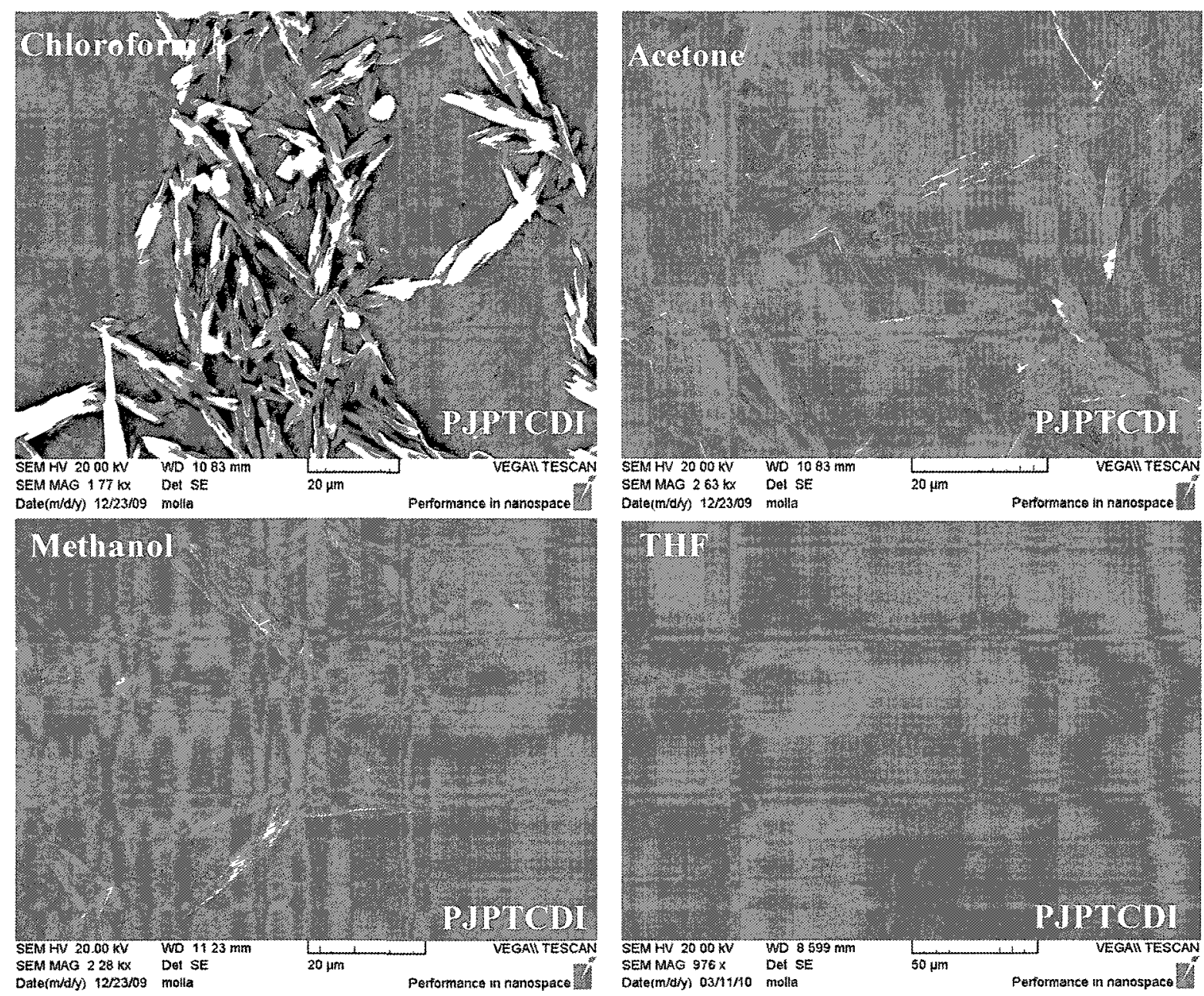

Figures continued in the next page 


\section{b}
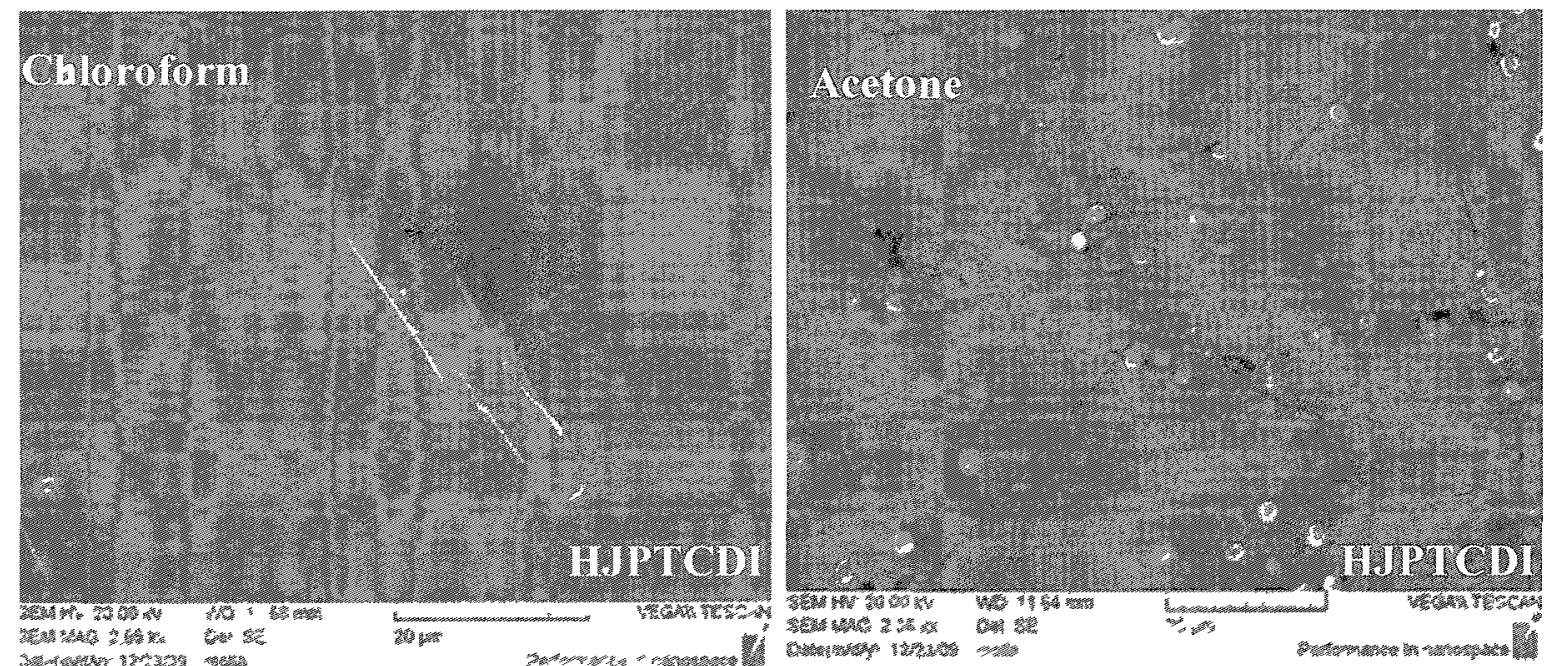

Sor

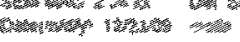

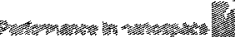
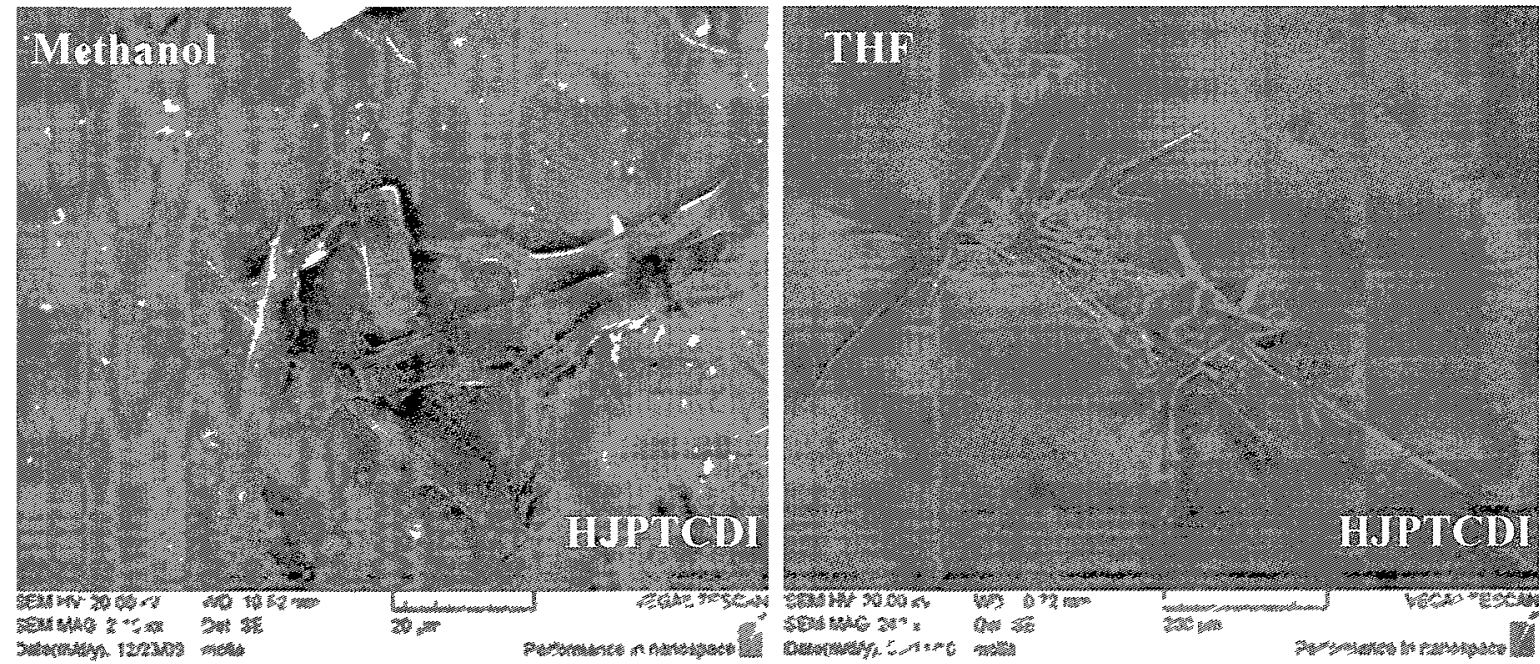

Figures continued in the next page 


\section{C}
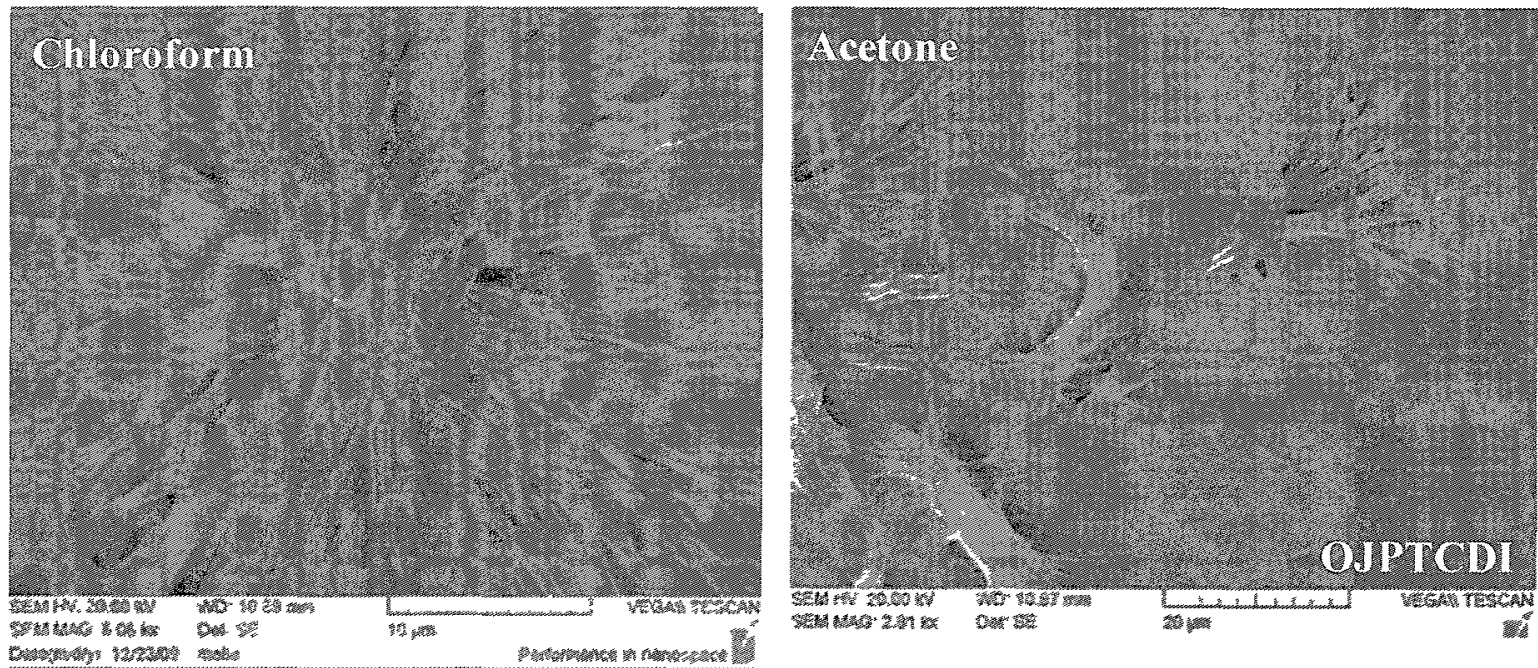

horas

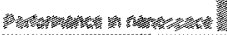
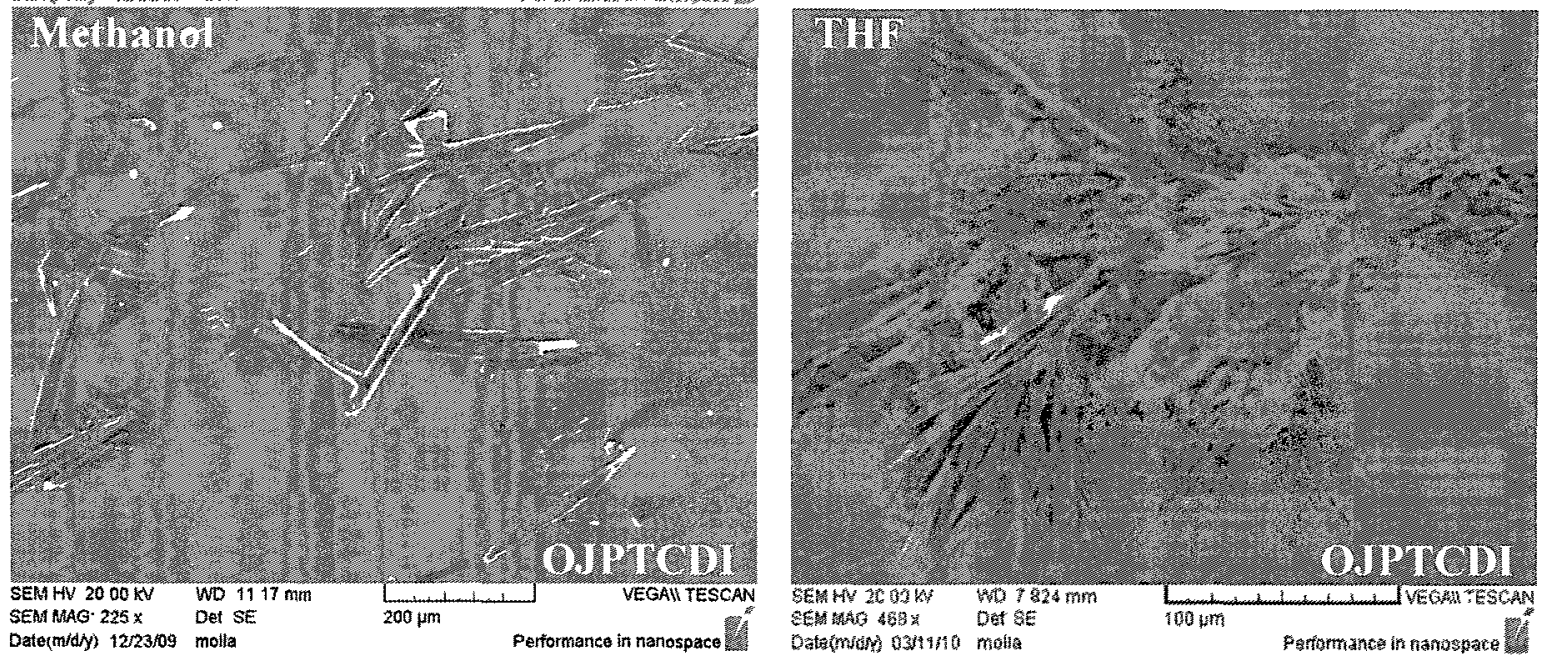

Figure 4.13: SEM images of (a) PJ-PTCDI, (b) HJ-PTCDI and (c) OJ-PTCDI self assembled from different solvents. 

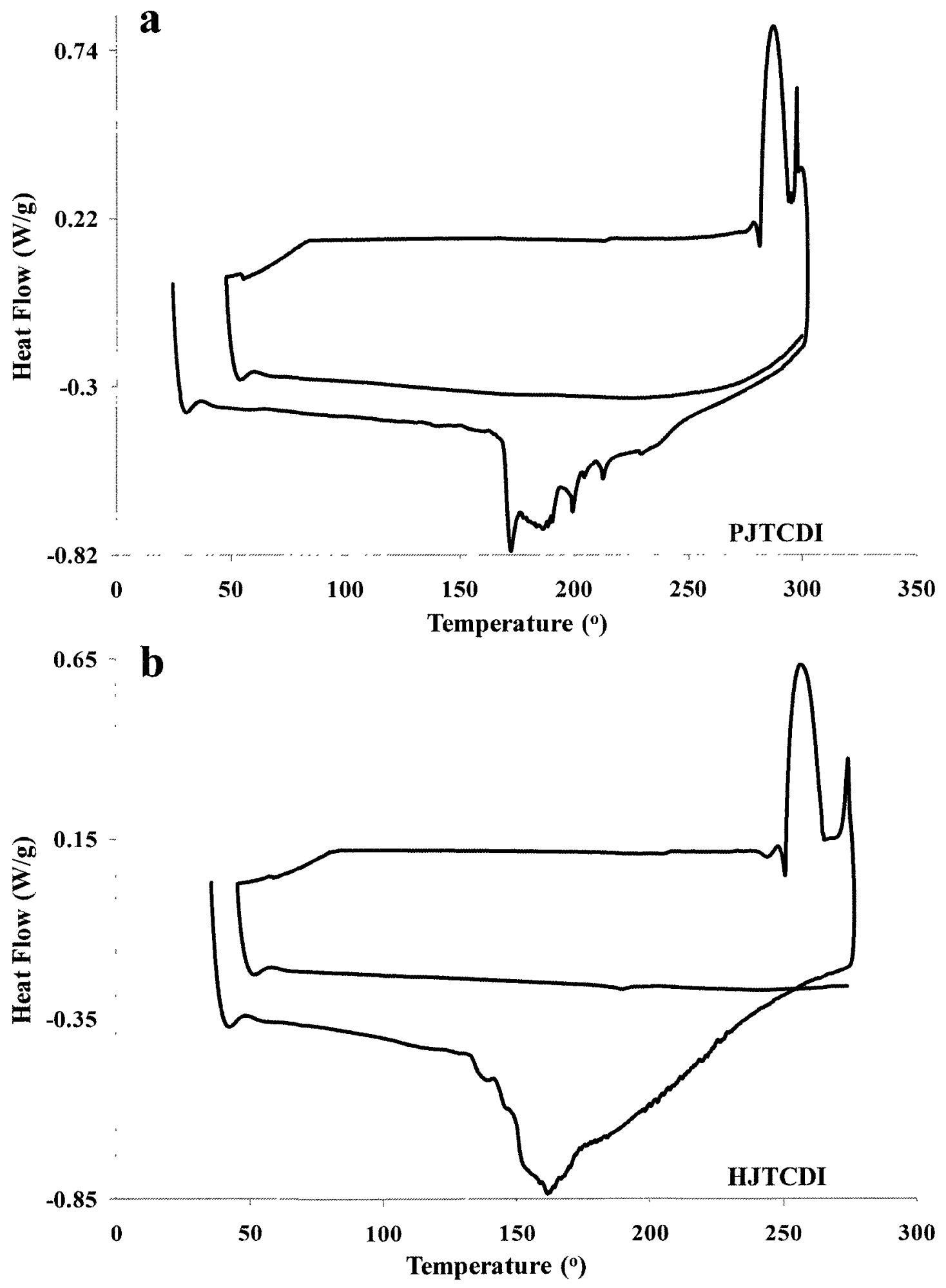

Figure continued in the next page 


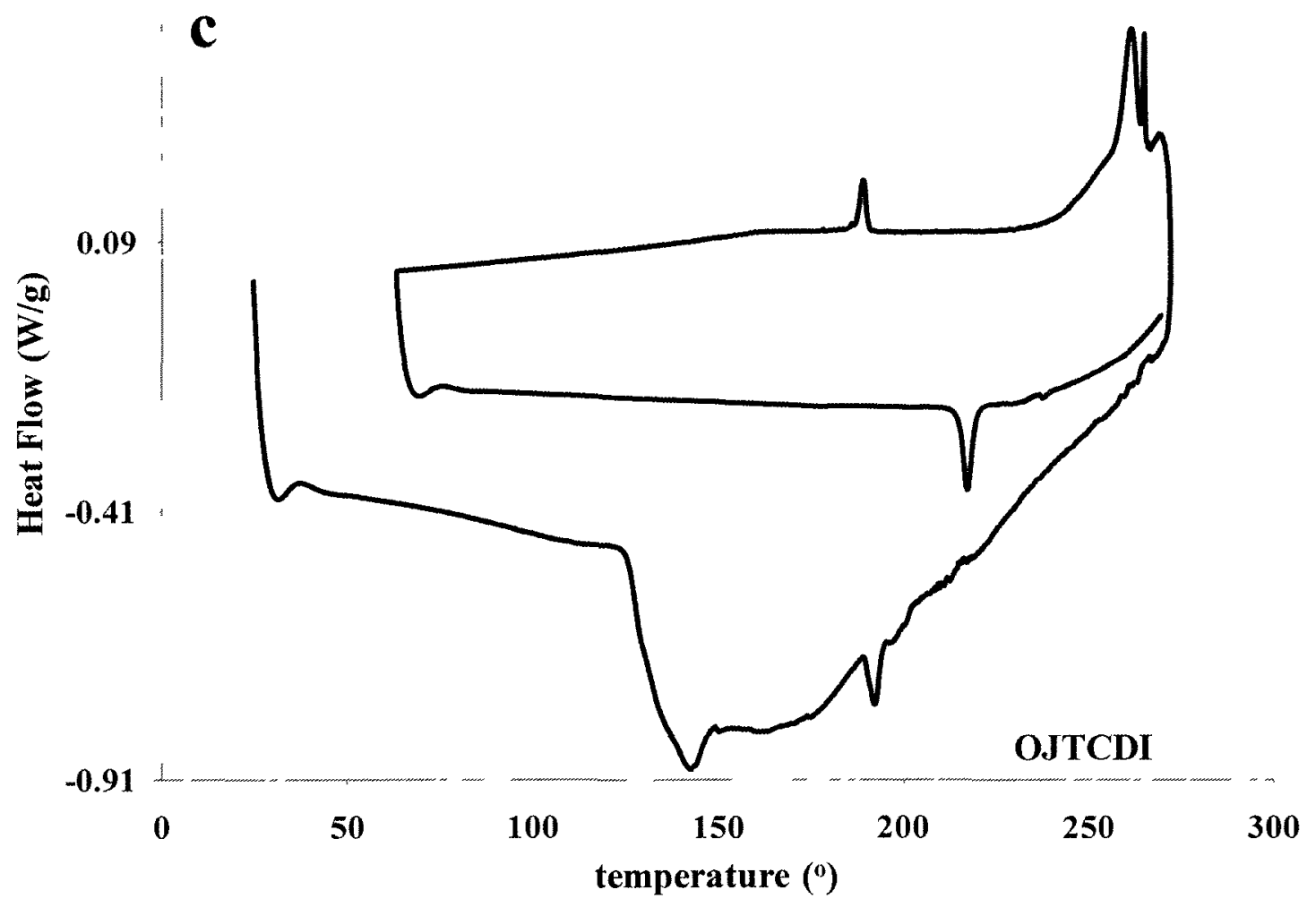

Figure 4.14: The DSC of (a) PJ-PTCDI, (b) HJ-PTCDI and (c) OJ-PTCDI.

\subsubsection{Thermal Analysis}

It is expected that the DSC of these PTCDIs would depend on the extent of packing of the perylene moiety. These materials did not show any liquid crystalline behavior (figure 4.14). PJ-PTCDI and HJ-PTCDI show irreversible melting over a broad range of temperatures (about 150 to $220^{\circ} \mathrm{C}$ ) and there was no crystallization observed in the cooling run. That these samples were initially crystalline was confirmed by X-ray diffraction (see below) and by the presence of aggregate crystals in the optical and scanning electron microscopy images. The broad melting could be attributed to a range of crystal sizes as well as lack regularity. The DSC trace of the OJ-PTCDI was different as it shows reversible transition at $189^{\circ} \mathrm{C}$ in the second cooling run. The first heating run 
was composed of a diffuse melting endotherm from $120-210{ }^{\circ} \mathrm{C}$ with a melting peak at $195^{\circ} \mathrm{C}$ which moved to $217^{\circ} \mathrm{C}$ in the second heating run. Thus, with an increase in the side chain length of the hydrophobic alkyl group the strength of the $\pi-\pi$ interaction increases and the crystals are more strongly packed. The peak in the cooling cycle for OJ-PTCDI confirms the strength of the aggregates.

\subsubsection{XRD Analysis:}

Well-developed peaks were seen in the XRD patterns for all the PTCDIs confirming their crystalline nature in the pure state, as seen earlier by POM images. The $d$-spacings are different for the three diimides, indicating that the packing mode varies amongst them. We see the most intense peaks in the range of $2 \theta=3.55^{\circ}$ to $4.50^{\circ}$ with $\mathrm{d}$ spacings of $19.83,24.20$ and $22.55 \AA$ for OJ-PTCDI, HJ-PTCDI and PJ-PTCDI respectively (Figure 4.15). A diffuse halo is seen from $2 \theta=15^{\circ}-25^{\circ}$ which is due to the Jeffamine chain in all the diimides. The $d$-spacing values observed for OJ-PTCDI, HJPTCDI and PJ-PTCDI are listed in table 4.1. The $d$-spacing values for OJ-PTCDI include peaks with d-values 6.70 and 7.52 with their second order reflection at 3.36 and $3.75 \mathrm{~A}^{\circ}$, intense peak at 4.48 some and most intense peak with d value 19.83 with its second order reflection at $\mathrm{d} 9.98 \mathrm{~A}^{\circ}$. The second-order reflection peaks in case of OJPTCDI confirm the very organized structure of the aggregates. Another feature seen in figure 4.15 is that a single most intense reflection is seen (especially for OJ-PTCDI) and the other reflections are very weak. This indicates predominantly one dimensional order due to the stacking of the aromatic segment. 
Table $4.1 d$-spacing values of X-ray profile of PJ-PTCDI, HJ-PTCDI and OJPTCDI. Here strong, medium and weak reflections are marked as s, $\mathrm{m}$, and $\mathrm{w}$.

\begin{tabular}{|c|c|c|}
\hline $\begin{array}{c}\text { PJ-PTCDI } \\
d \text {-spacing values }\end{array}$ & $\begin{array}{c}\text { HJ-PTCDI } \\
d \text {-spacing values }\end{array}$ & $\begin{array}{c}\text { OJ-PTCDI } \\
\text {-spacing values }\end{array}$ \\
\hline $\mathbf{2 2 . 5 5}(\mathbf{s})$ & $\mathbf{3 3 . 3 7}(\mathbf{s})$ & $\mathbf{1 9 . 8 3}(\mathbf{s})$ \\
\hline $\mathbf{1 1 . 1 7}(\mathbf{s})$ & $29.63(\mathrm{w})$ & $9.98(\mathrm{w})$ \\
\hline $\mathbf{8 . 1 4}(\mathbf{s})$ & $\mathbf{2 4 . 2 0 ( \mathbf { s } )}$ & $7.52(\mathrm{w})$ \\
\hline $\mathbf{7 . 7 5}(\mathbf{s})$ & $14.33(\mathrm{w})$ & $6.70(\mathrm{~m})$ \\
\hline $7.20(\mathrm{w})$ & $12.77(\mathrm{w})$ & $4.48(\mathrm{~m})$ \\
\hline $6.67(\mathrm{~m})$ & $7.38(\mathrm{w})$ & $3.75(\mathrm{~m})$ \\
\hline $6.08(\mathrm{~m})$ & $6.17(\mathrm{~m})$ & $3.36(\mathrm{w})$ \\
\hline $3.48(\mathrm{w})$ & $3.37(\mathrm{w})$ & \\
\hline
\end{tabular}



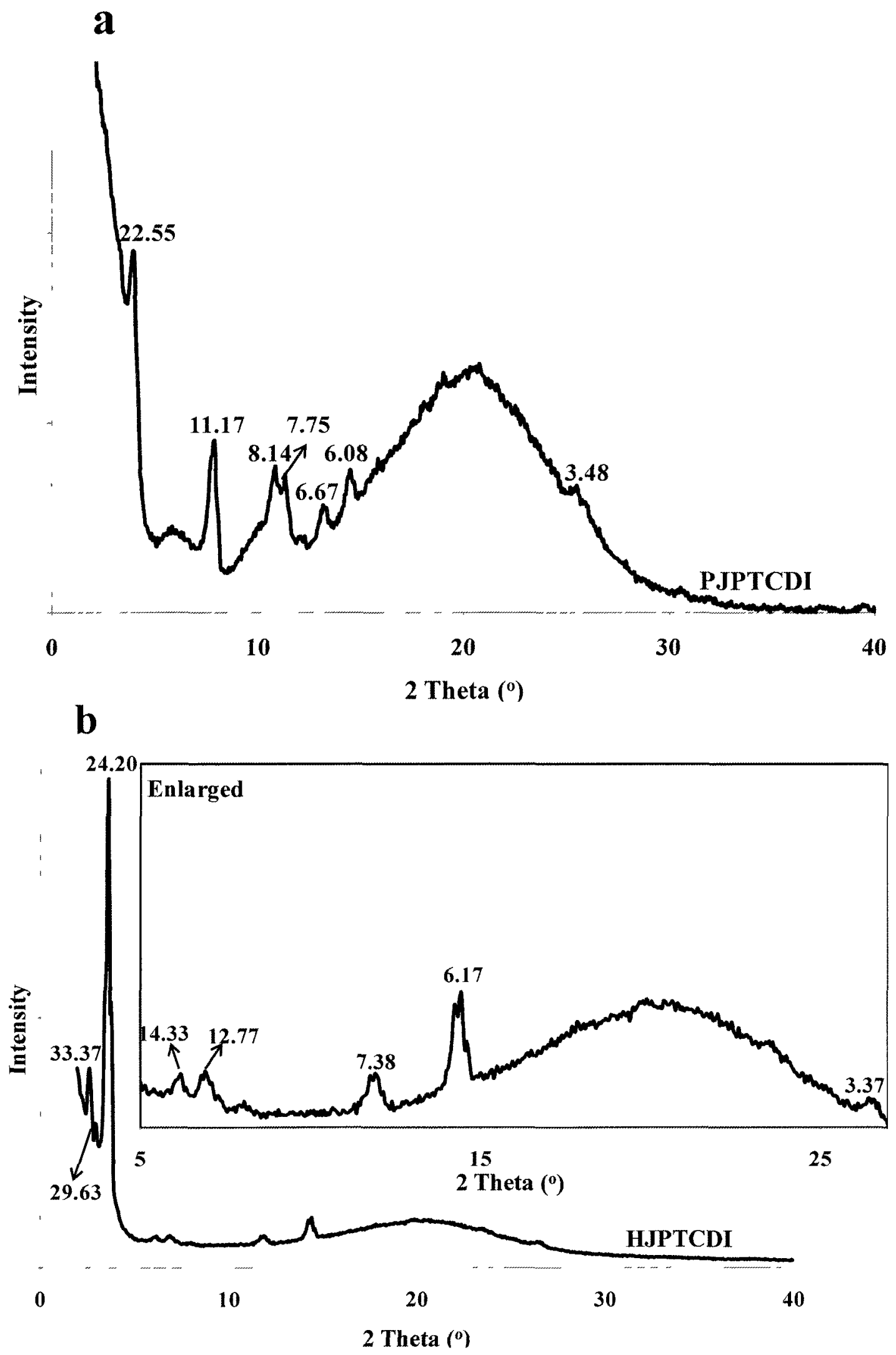

Figure continued in the next page 


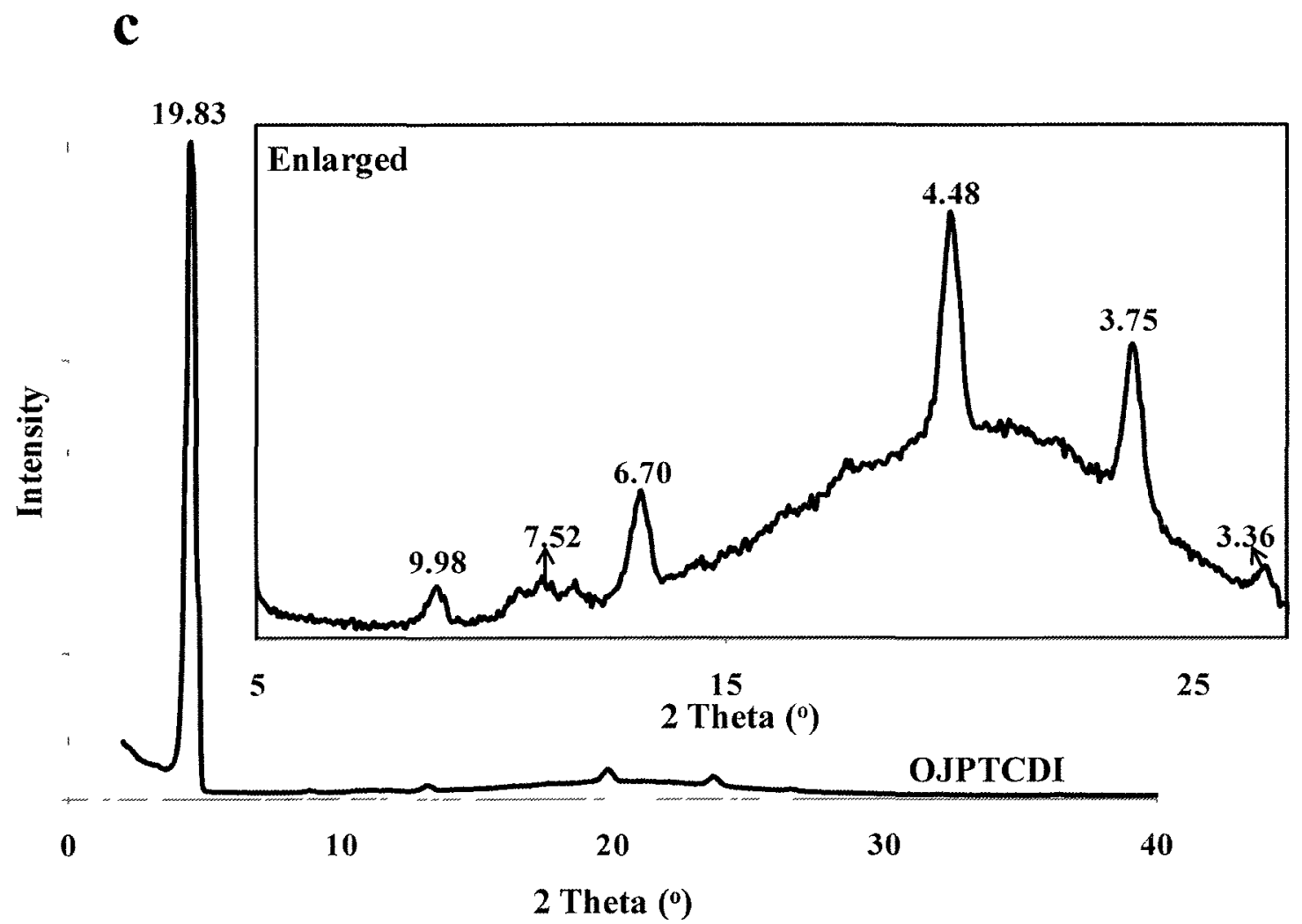

Figure 4.15: a) The X-ray profile of (a) PJ-PTCDI, (b) HJ-PTCDI and (c) OJ-PTCDI respectively. The XRD patterns were recorded by taking the pure compounds on the glass slides.

\subsection{Conclusions}

We have studied the effect of the length of the hydrophobic linear side chain on the packing and the morphology of the amphiphilic perylene diimide, which is like a Gemini surfactant. For the short linear alkyl chain we see the packing mode in aqueous solution starts reorganizing at higher concentration $\left(10^{-5} \mathrm{M}\right)$ whereas for longer chains the packing starts at concentrations as low as $10^{-7} \mathrm{M}$. The morphology of drop cast films consists of folded sheets and tubes. All the diimides are in a molecularly dissolved state (showing the characteristic absorption of perylene monomer) in less polar solvents at all 
concentrations. In a mixed solvent system (acetone-water or methanol-water mixture) PJPTCDI forms J-type aggregate whereas in water it aggregates as H-type. The extent of aggregation can be controlled by aqueous-non aqueous solvent mixtures. The aggregate structures show high thermal stability. The ordered structure of the perylene diimide is very important for potential applications in devices such as the solar cell and optoelectronics. We have shown that control over aggregation of the diimides is possible by selectively changing the hydrophobic side chain length and the solvents. The water based aggregation is an advantage. 


\subsection{References:}

1. Ghadiri, M. R.; Granja, J. R.; Milligan, R. A.; McRee, D. E.; Khazanovich, N. Nature 1993. 366,324 .

2. Rosenthal-Aizman, K.; Svensson, G.; Unden, A. J. Am. Chem. Soc. 2004, 126, 3372.

3. Hartgerink, J. D.; Beniash, E.; Stupp, S. I. Science 2001, 294, 1684.

4. Niece, K. L.; Hartgerink, J. D.; Donners, J. J. J. M.; Stupp, S. I. J. Am. Chem. Soc. $2003,125,7146$.

5. Bushey, M. L.; Nguyen. T.-Q.; Nuckolls, C. J. Am. Chem. Soc. 2003, 125, 8264.

6. Bushey, M. L.; Hwang, A.; Stephens, P. W.; Nuckolls, C. Angew. Chem. Int. Ed. 2002, 41,2828 .

7. Nguyen, T.-Q.; Bushey. M. L.; Brus, L. E.; Nuckolls, C. J. Am. Chem. Soc. 2002, 124 , 15051.

8. Nguyen, T.-Q.; Martel, R.; Avouris, P.; Bushey, M. L.; Brus, L.; Nuckolls, C. J. Am. Chem. Soc. 2004, 126, 5234 .

9. Hill, J. P.; Jin, W.; Kosaka, A.; Fukushima, T.; Ichihara, H.; Shimomura, T.; Ito, K.; Hashizume, T.; Ishii, N.; Aida, T. Science 2004, 304, 1481.

10. Pisula, W.; Kastler, M.; Wasserfallen, D.; Pakula, T.; Müllen, K. J. Am. Chem. Soc. 2004, 126,8074 .

11. Schwab, A. D.; Smith, D. E.; Rich, C. S.; Young, E. R.; Smith, W. R.; de Paula, J. C. J. Phys. Chem. B 2003, 107, 11339.

12. Schwab, A. D.; Smith, D. E; Bond-Watts, B.; Johnston, D. E.; Hone, J.; Johnson, A. T.; de Paula, J. C.; Smith. W. F. Nano Lett. 2004, 4. 1261. 
13. Harada, R.; Matsuda, Y.; Okawa, H.; Kojima, T. Angew. Chem., Int. Ed. 2004, 43, 1825.

14. Huang, J.; Virji, S.; Weiller, B. H.; Kaner, R. B. J. Am. Chem. Soc. 2003, 125, 314.

15. Huang, J.; Kaner, R. B. J. Am. Chem. Soc. 2003, 126, 851.

16. Li, W.; Wang, H.-L. J. Am. Chem. Soc. 2004, 126, 2278.

17. Engelkamp, H.; Middelbeek, S.; Nolte, R. J. M. Science 1999, $284,785$.

18. von Berlepsch, H.; Bottcher, C.; Dahne, L. J. Phys. Chem. B 2000, 104, 8792.

19. Mishra, A.; Behera, R. K.; Behera, P. K.; Mishra, B. K.; Behera, G. B. Chem. Rev. 2000, 100, 1973.

20. Wurthner, F.; Yao, S.; Beginn, U. Angew. Chem., Int. Ed. 2003, 42. 3247.

21. Yao. S.; Beginn, U.; Gress, T.; Lysetska, M.: Würthner, F. J. Am. Chem. Soc. 2004, 126.8336 .

22. (a) Yagai, S.; Ishii, M.; Karatsu, T.; Kitamura, A. Angew. Chem. Int. Ed. 2007, 46, 8005. (b) Ryu, J.-H.; Hong, D.-J.; Lee, M. Chem. Commun. 2008, 9,1043.

23. Sui, G.; Orbulescu, J.; Mabrouki, M.; Leblanc, R.M.: Liu. S.; Gregg. B.A. Chem phys chem, 2002, 12.1041.

24. Chen, Z.; Baumeister, U.; Tschierske, C.; Wurthner, F. Chem. Eur. J. 2007, 13, 450.

25. Weitz, R. T.; Amsharov, K.; Zschieschang, U.; Villas, E. B.; Goswami,D. K.; Burghard, M.; Dosch, H.; Jansen, M.; Kern, K.; Klauk, H. J. Am. Chem. Soc., 2008, 130, 4637.

26. Tatemichi, S.; Ichikawa, M.; Koyama, T.; Taniguchi, Y. Appl. Phys. Lett. 2006, 89. $112108(1-3)$ 
27. Horowitz, G.; Kouki, F.: Spearman, P.; Fichou, D.; Nogues, C.; Pan, X.; Garnier, F. Adv. Mater. 1996, 8, 242.

28. Laquindanum, J. G.;Kartz, H. E.; Dodabalapur, A.; Lovinger, A. J. J. Am. Chem. Soc. 1996, $118,11331$.

29. Malenfant, P. R. L.; Dimitrakopoulos, C. D.; Gelorme, J. D.; Kosbar, L. L.; Graham, T. O.: Curioni, A.; Andreoni, W. Appl. Phys. Lett. 2002, 80, 2517.

30. Gundlach, D. J.; Pernstich, K. P.; Wilckens, G.; Gruter, M.; Haas, S.; Batlogg, B. J. Appl. Phys. 2005, 98, 064502(1-8).

31. Wang, Y.; Chen, Y.; Li, R.; Wang, S.; Su, W.; Ma, P.; Wasielewski, M. R.; Li, X.; Jiang, J. Langmuir 2007, 23, 5836.

32. Newman, C. R.; Frisbie, C. D.; da Silva Filho, D. A.; Bredas, J.-L.; Ewbank, P. C.; Mann, K. R. Chem. Mater. 2004, 16, 4436.

33. Xu, B.; Xiao. X.; Yang, X.; Zang, L.; Tao, N. J. Am. Chem. Soc. 2005. 127, 2386.

34. van Herrikhuyzen, J.; Syamakumari, A.; Schenning, A. P. H. J.; Meijer, E. W. J. Am. Chem. Soc. 2004, 126, 10021.

35. He, X.; Liu, H.; Wang, N.; Ai, X.; Wang, S.; Li, Y.; Huang, C.; Cui, S.; Li, Y.; Zhu, D.; Macromol. Rapid Commun. 2005, 26, 721.

36. Balakrishnan, K.; Datar, A.; Naddo, T.; Huang, J.; Oitker, R.; Yen, M.; Zhao, J.; Zang, L. J. Am. Chem. Soc. 2006, 128, 7390.

37. Balakrishnan, K.; Datar, A.; Oitker, R.; Chen, H.; Zuo, J.; Zang, L. J. Am. Chem. Soc. $\mathbf{2 0 0 5}, 127,10496$.

38. Datar, A.; Balakrishnan, K.; Yang, X.; Zuo, X.; Huang, J.; Oitker, R.; Yen, M.; Zhao, J.; Tiede, D. M.; Zang L. J. Phys. Chem. B 2006, 110, 12327. 
39. Datar, A.; Oitker R.; Zang, L. Chem. Commun. 2006, 15, 1649.

40. Yan, P.; Chowdhury, A.; Holman, M. W.; Adams, D. M. J. Phys. Chem. B 2005, 109, 724.

41. Everett, T. A.; Twite, A.A.; Xie, A.; Battina, S. K.; Hua, D. H.; Higgins, D.A. Chem. Mater. 2006, 18, 5937.

42. Shi, M.-M.; Chen, H.-Z.; Shi, Y.-W.; Sun, J.-Z.; Wang, M. J. Phys. Chem. B 2004, 108,5901

43. Ng, A. M. C.; Tong, W. Y.; Djurisic, A. B.; Chan, W. K. Proc. of SPIE, Nanophotonic Materials III, 2009, 6321, 63210N (1-8).

44. Bai, R.; Ouyang, M.; Zhou, R.-J.; Shi, M.-M.; Wang, M.; Chen, H.-Z. Nanotechnology, 2008, 19, 055604(1-6).

45. (a) Stohr, M.; Wahl, M.; Galka, C. H.; Riehm, T.; Jung, T. A.; Gade, L. H. Angew. Chem. Int. Ed. 2005, 44, 7394. (b) Tang, T.; Qu, J.; Mullen, K.; Webber, S. E. Langmuir 2006, 22, 26. (c) del Cano, T.; Parra, V.; Rodriguez-Mendez, M.L.; Aroca, R.; de Saja, J.A.; Organic Electronics, 2004, 5, 107. (d) Yao, D.; Bender, T. P.; Gerroir, P. J.; Sundararajan, P.R. Macromolecules 2005. 38, 6972. (e) Yao, D.; Tuteja, B.; Sundararajan, P. R. Macromolecules 2006, 39, 7786. (f) Zhang, X.; Chen, Z.; Wurthner, F. J. Am Chem Soc. 2007, 129, 4886.

46. Nolde, F.; Pisula, W.; Muller, S.; Kohl, C.; Mullen, K. Chem. Mater. 2006, 18, 3715. 47. Zhan, X.; Tan, Z.; Zhou, E.; Li, Y.; Misra, R.; Grant, A.; Domercq, B.; Zhang, X.-H.; An, Z.; Zhang, X.; Barlow, S.; Kippelenc, B.; Marder, S. R. J. Mater. Chem., 2009, 19, 5794. 
48. He, X.; Liu, H.; Li, Y.; Liu, Y.; Lu, F.; Li, Y.; Zhu, D. Macromol. Chem. Phys. 2005, $206,2199$.

49. Yang, X.; Xu, X.; Ji, H.-F. J. Phys. Chem. B 2008, 112, 7196.

50. Wang, W.; Wang, L.Q.; Palmer, B. J.; Exarhos, G. J.; Li, A. D. Q. J. Am. Chem. Soc. 2006, 128. 11150.

51. Kasha, M.; Rawls, H. R.; El-bayoumi, M. A. Pure Appl. Chem. 1965, 11, 371.

52. Clark, A. E.; Qin, C.; Li , A. D. Q. J. Am. Chem. Soc. 2007, 129, 7586.

53. Wang, W.; Han, J. J.; Wang, L.-Q.; Li, L.-S.; Shaw,W. J.; Li, A. D. Q. Nano Lett., $\mathbf{2 0 0 3}, 3.455$.

54. Wurthner, F.; Chen, Z.; Hoeben, F. J. M.; Osswald, P.; You, C.-C.; Jonkheijm, P.; Herrikhuyzen, J. v.; Schenning, A. P. H. J.; van der Schoot, P.P. A. M.; Meijer, E. W. Beckers, E. H. A.; Meskers, S. C. J.; Janssen, R. A. J. J. Am. Chem. Soc. 2004, 126, 10611.

55. Langhals, H.; Jona, W. Angew. Chem. Int. Ed. 1998, 37, 952.

56. Kim, O.-K., Je, J., Jernigan, G., Buckley, L., and Whitten, D. J. Am. Chem. Soc. 2006, 128,510 .

57. Li, A. D. Q.; Wang, W.; Wang, L.-Q. Chem. Eur. J. 2003, 9, 4594.

58. Wurthner, F.; Thalacker, C.; Diele, S.; Tschierske, C.; Chem. Eur. J. 2001, 7, 2245.

59. Langhals, H.; Ismael, R. Eur. J. Org. Chem. 1998, 9, 1915.

60. Chen, Z.; Baumeister, U.; Tschierske,C.; Wurthner, F. Chem. Eur. J. 2007, 13, 450.

61. Khanna, Shalini.; Khan, M. K.; Sundararajan, P. Langmuir 2009, 25, 13183. 
CHAPTER V

Self-assembly of Peylene Bisimide Jacketed by Polystyrene. 


\subsection{Introduction}

Self-assembly needs the presence of self assembling codes and the driving force to guide the process in different organic or biological molecular systems, and the initiation is often triggered by the interaction of solvents. ${ }^{1-14}$ While perylene diimide is notoriously insoluble in common solvents, derivatization eliminates this shortcoming to make it suitable for different applications ${ }^{15-19}$ and in some cases offers liquid crystalline

property as well. $^{20,21}$ The substitution in perylene can be done on imide nitrogen or on the peripheral positions. Depending on the position and nature of the substituents, the mode of self-assembly would be different. In chapters III and IV we discussed the aqueous self-assembly of amphiphilic type perylene diimide based block copolymer where we attached hydrophilic jeffamine ${ }^{\circledR}$ in either sides of imide nitrogen or one side of imide nitrogen with jeffamine and the other side with alkyl groups of different lengths. In this aspect we have discussed the self-assembly, stability and morphology of aggregates and found a way to tune the morphology by modulating the hydrophobic side group of perylene diimide. To explore non aqueous based self-assembly in our previous studies we reported vesicular morphology ${ }^{22}$ for a system of polydimethylsiloxane-coperylenemonoanhydride (PDMS-co-PTCDI) where PDMS was attached to one side of perylene. This could be considered a pseudo di-block copolymer, in which the PTCDI forms the short segment. This could also be viewed as a macromolecular inverse surfactant applicable to non-aqueous systems. Similar to the case of amphiphilic block copolymer systems, micellar or vesicular morphologies were observed, ${ }^{22}$ depending on the solvent used. A similar pseudo tri-block copolymer, PDMS-co-PTCDI-co- PDMS 
formed nano-web morphology. ${ }^{23}$ In our present chapter we will discuss polystyrene based perylene diimide.

The PDMS is a waxy polymer with a low glass transition temperature $\left(T_{g}\right)$ of $120^{\circ} \mathrm{C}$. In this chapter, we attached polystyrene, which has a $\mathrm{T}_{\mathrm{g}}$ of about $60^{\circ} \mathrm{C}$ to both sides of the PTCDI. Polystyrene is widely used in studies of block copolymer selfassembly. This unique combination of a flexible polymer and a rigid extended $\pi$-electron system (PS-PTCDI-PS) could lead to interesting morphologies. A solvent can be considered as an additional parameter, which normally increases the transition dipole moment (the oscillator length) and/or broadens the spectra leading to a loss in vibronic structure. In the case of perylene diimide, absorption spectra in aliphatic solvents show less influence with well-resolved vibronic fine structure. On the other hand polar and protic ones interact more with the solute molecule. A poor solvent for one of the blocks helps the self-assembly. A mixture of solvents can guide the self-assembly in a very specific way. Hence, in the case of perylene diimide the self-assembly is a complex process which comprises the nature of solvent(s), nature of side chain (extended or coiled), concentration, temperature and thermodynamic stability, presence of one or more self-assembly codes etc. ${ }^{24-28}$

Here we discuss a study of the self-assembly of PS-PTCDI-PS in different solvents. The short hydrophobic polystyrene is used as the peripheral side chain. Polystyrene has a planar $\pi$ bonding moiety to give additional support to the $\pi-\pi$ interaction of the perylene units. Different morphologies varying from nanosphere to porous sheets of drop cast films are seen, depending on the solvents and solvent mixtures. 


\subsection{Experimental:}

3, 4, 9, 10-Perylenetrtracarboxylic acid dianhydride, dimethyl acetamide (DMAc) and zinc acetate dihydrate were purchased from Aldrich Chemical Company and used as received. Amine terminated polystyrene (average molecular weight 1200) was purchased from Polymer Source Inc. and used as received. Chloroform was purchased from Caledon Laboratories Ltd. and the other solvents were purchased from Aldrich Chemical Company Inc.

${ }^{\prime} \mathrm{H}$ NMR spectra were recorded on a Bruker-400 spectrometer using tetramethylsilane as an internal reference in chloroform. Infrared measurements were performed either on a Michelson M120 BOMEM FTIR spectrometer or Varian 1000 Scimitar Series spectrometer in which $\mathrm{KBr}$ pellets were used for the powder samples and $\mathrm{NaCl}$ discs were used for the solution samples. Normally the samples were dissolved in chloroform to make a dilute solution and then coated on $\mathrm{NaCl}$ discs. X-ray diffraction (XRD) data were collected using a Philips automated powder diffractometer model PW 1710 and nickel filtered $\mathrm{Cu} \mathrm{K} \alpha$ radiation $(\lambda=1.542 \AA)$. The MDI Datascan 3.2 software (Materials Data Inc., Livermore, CA) was used for data collection. The results were analyzed with MDI Jade 5.0 XRD pattern processing software.

A Zeiss Axioplan 2 imaging universal optical microscope with Q-imaging Retiga digital camera was used to record optical micrographs both in polarized and reflection modes. Northern Eclipse (version 6.0) image processing software was used. Scanning electron micrographs (SEM) were recorded with a JSM-6400 scanning electron microscope or a Tescan Vega-II XMU VPSEM. Samples were prepared by drop casting 
solutions in different solvents and concentrations on a glass slide or on a SEM pin mount holder. The surface of the samples for SEM (on either glass slide or pin metal holder) was coated with gold/ palladium (80/20\%) alloy layer by HUMMER VII Sputtering System (ANATECH Ltd, Alexandria, VA.) to make it conductive. The thickness of the conductive layer was $9-10 \mathrm{~nm}$.

Thermal analysis was performed using a TA Instrument 2010 differential scanning calorimeter at a heating rate of $10{ }^{\circ} \mathrm{C} / \mathrm{min}$. The samples were cooled to room temperature at a cooling rate of $5{ }^{\circ} \mathrm{C} / \mathrm{min}$ and $2^{\circ} \mathrm{C} / \mathrm{min}$ in the second run. This slow rate of cooling was used to detect any small exothermic change. The instrument was calibrated for temperature and energy with indium and tin reference samples. DSC traces were recorded with about 7-10 $\mathrm{mg}$ of sample in an aluminum pan.

The UV-Vis spectroscopic measurements were performed at ambient conditions using a Varian CARY 3 UV-Vis spectrophotometer. The path length of the quartz sample cube was $10 \mathrm{~mm}$. Temperature dependent UV-Vis spectra were recorded with a Cary 300 Bio UV-Vis spectrophotometer attached with a temperature controller (operating temperature $-10^{\circ} \mathrm{C}$ to $100^{\circ} \mathrm{C}$ ) by Varian. The data was processed by Cary WinUV Software version 3.00 (339). Fluorescent spectra were taken by Varian Cary Fluorescent spectrophotometer. Data collection and handling were done by Eclipse Software version 1.1 (132). Fluorescent Spectra were recorded at $\lambda_{\mathrm{ex}} 520 \mathrm{~nm}$ with a fixed bandwidth of 5 $\mathrm{nm}$ for excitation and $5 \mathrm{~nm}$ for emission. 


\subsubsection{Synthesis of 3, 4, 9, 10-perylenetetracarboxylic Diimide of Polystyrene}

\section{(PS-PTCDI-PS)}

The title compound was prepared by the procedure published by Jancy and Asha ${ }^{29}$ (scheme 5.1) with a slight modification. (These authors washed the slurry of product with hexane and/or acetone and then purified by column chromatography. In our case, we used the solvent extraction method with a mixture of water and chloroform $/ \mathrm{CH}_{2} \mathrm{Cl}_{2}$ and then dried in vacuum oven to get the product).

In a round-bottom flask amino terminated polystyrene $(0.40 \mathrm{~g}, 0.33 \mathrm{mmol})$, zinc acetate $(0.001 \mathrm{~g}, 0.006 \mathrm{mmol})$ in $N, N$-dimethylacetamide $(25 \mathrm{~mL})$ were heated in an oil bath attached with a condenser for 1 hour with stirring at $110{ }^{\circ} \mathrm{C}$. $3,4,9,10$ perylenetetracarboxylic dianhydride $(0.03 \mathrm{~g}, 0.08 \mathrm{mmol})$ was added, and the reaction mixture was stirred vigorously at $110^{\circ} \mathrm{C}$ for $3 \mathrm{~h}$ and then continued at $160{ }^{\circ} \mathrm{C}$ for another $16 \mathrm{~h}$ keeping the same set up. The excess solvent was distilled off, and the slurry was washed several times with benzene to remove unreacted amino terminated polystyrene and dissolved in chloroform. The solution was filtered to remove unreacted 3, 4, 9, 10perylenetetracarboxylic dianhydride if any. The chloroform solution was extracted with acidic distilled water three times and then with distilled water a few more times until the aqueous phase $\mathrm{pH}$ is neutral. The extracted chloroform solution was dried over sodium sulphate. The solution was then evaporated to get the final product, which was kept in a vacuum chamber for $24 \mathrm{hrs}$ to remove any solvent and dried to get a red product (yield 78\%). The FTIR spectra revealed the presence of imide carbonyl absorption frequencies (1697 and $1655 \mathrm{~cm}^{-1}$ ) confirming the formation of the compound (Appendix A, figure 
A8) as a diimide of PS. ${ }^{~} \mathrm{HNMR}$ spectra also confirmed the presence of perylene group giving a multiplet at around $8.6 \mathrm{ppm}$ (Appendix B, figure B7).

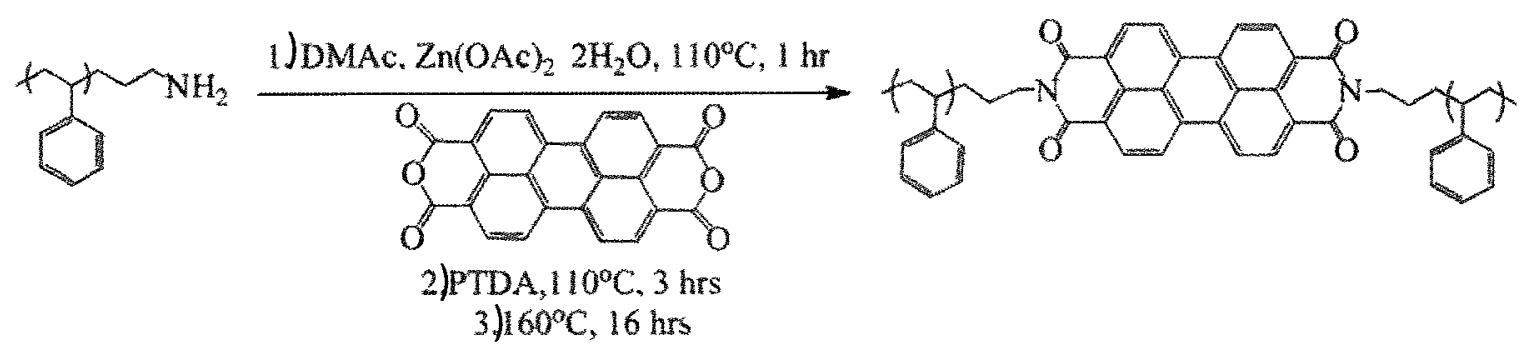

Scheme 5.1: Reaction of amino terminated polystyrene with perylene dianhydride to give coil-rod-coil block copolymer, PS-b-Perylene-b-PS.

\subsection{Results and Discussion}

\subsubsection{Self-assembly in Solution Studied by Absorption and Fluorescence Spectroscopy}

Amino terminated polystyrene used for this study is soluble in THF, chloroform and toluene whereas it precipitated out of methanol and hexane. The rigid perylene unit is well known for its insolubility in almost all solvents. The absorption spectra (figure 5.1a) of PS-PTCDI-PS in all of the non-aqueous solvents studied here at $10^{-4} \mathrm{M}$ shows the typical absorption pattern of the non-aggregate form..$^{3.4 .8}$ The long wavelength peak is the most intense, with the peak intensity decreasing at lower wavelengths. The $\lambda_{\max }\left(\mathrm{S}_{0}-\mathrm{S}_{0}\right)$ varies from 519-525 $\mathrm{nm}$ with different solvents which can be explained as the stabilization of the ground state of the perylene moiety in these solvents with different polarities. In toluene the $\mathrm{S}_{0.0}$ transition occurs at longer wavelength whereas in acetone the shift is towards shorter wavelength. The $\mathrm{S}_{0-1}$ and $\mathrm{S}_{0-2}$ transition peaks are seen in the range $484-490$ and $456-459 \mathrm{~nm}$ respectively. Concentration does not have any effect on the lack of aggregation. 

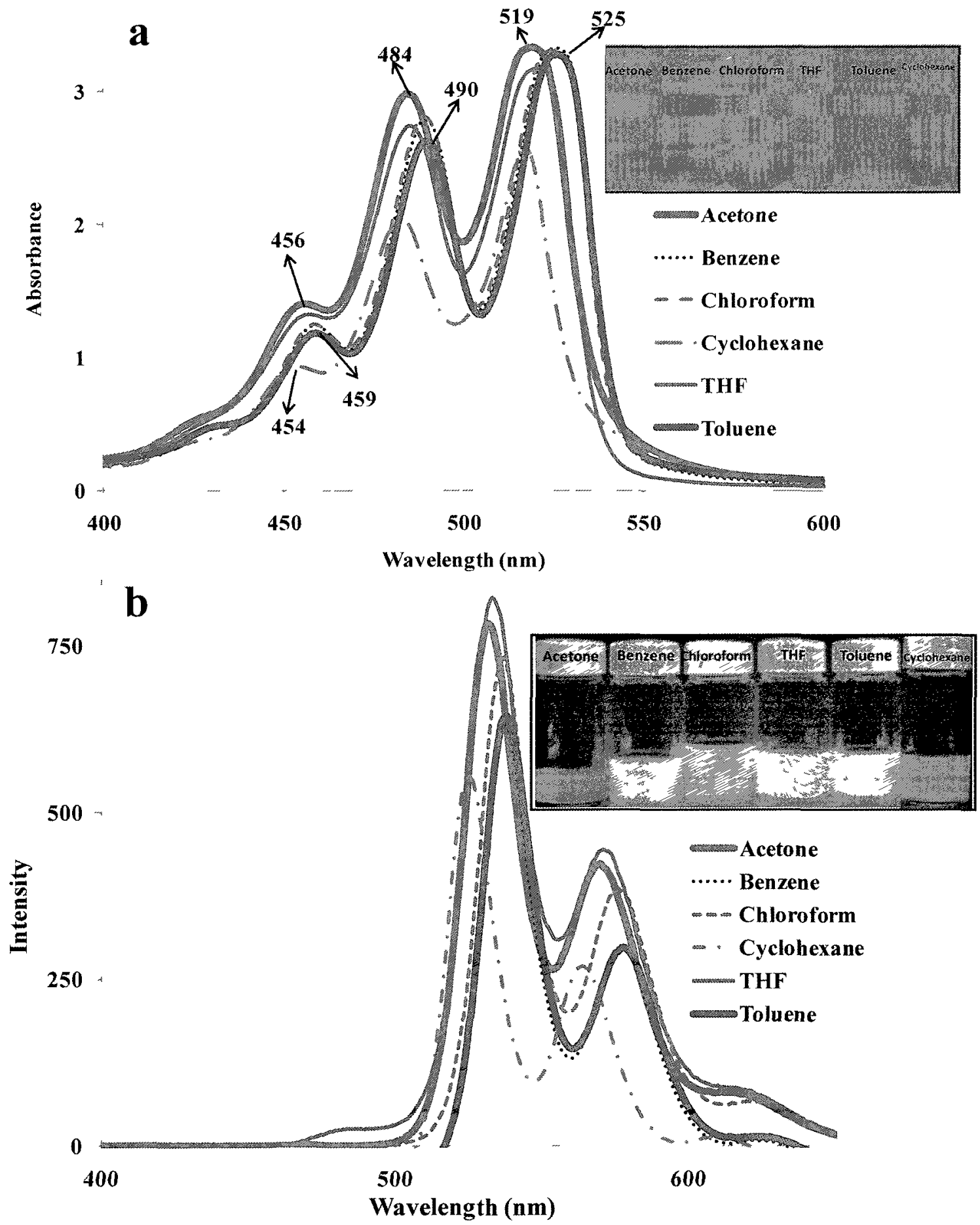

Figure 5.1: UV-Vis (a) and fluorescence (b) spectra of PSPTCDIPS in different solvents at $10^{-4} \mathrm{M}$. The inset of each figure shows the solution without and with excitation at 366 nm. 


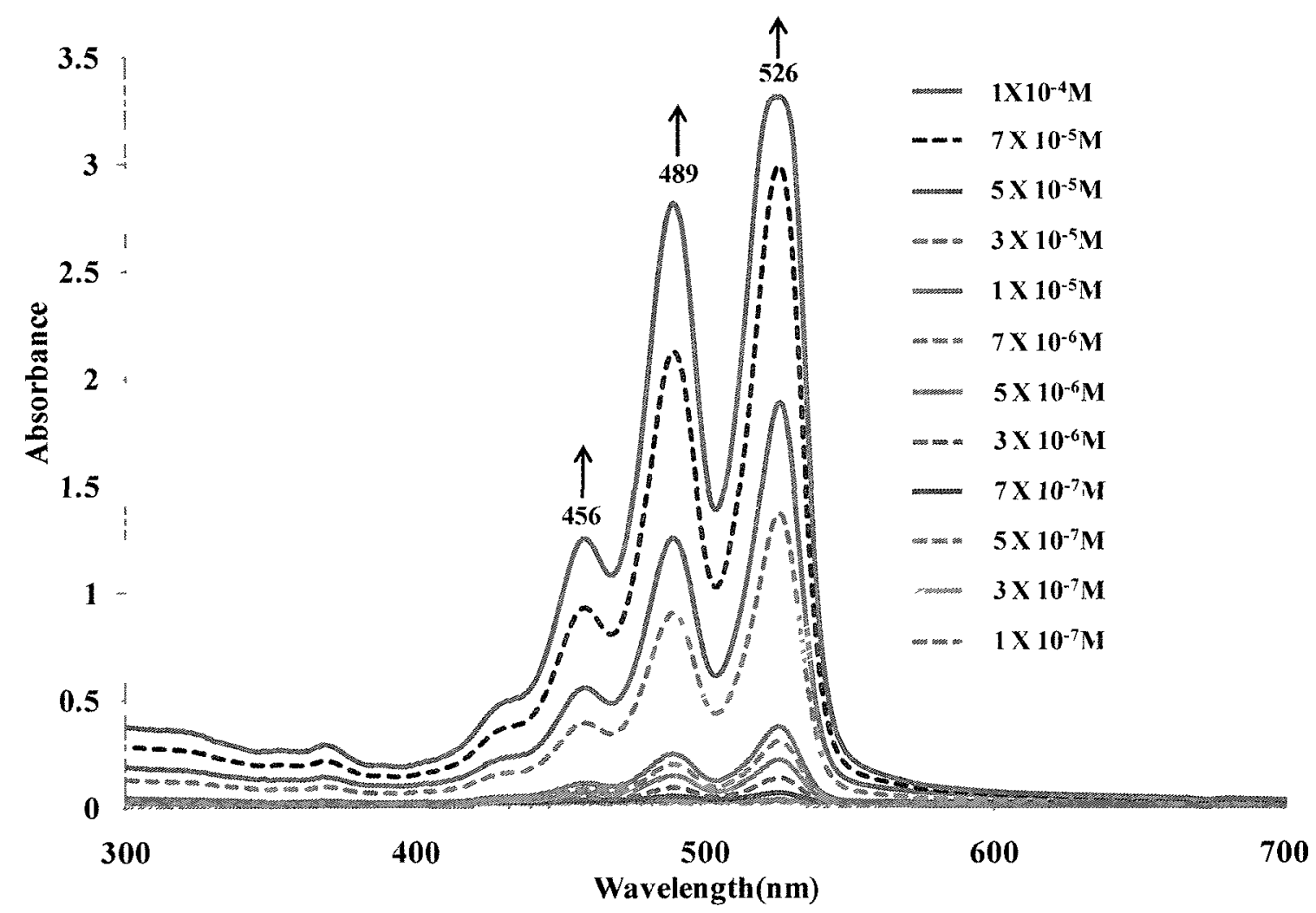

Figure 5.2: UV-Vis and fluorescence spectra of PSPTCDI PS in chloroform from $10^{-7}$ to $10^{-4} \mathrm{M}$.

It is seen from figure 5.2 that the shape of the absorption spectra is the same with a concentration range from $10^{-7} \mathrm{M}$ to $10^{-4} \mathrm{M}$ in chloroform and shows the typical monomeric absorption pattern. The peak positions originating from $S_{0-2}, S_{0-1}$ and $S_{0-0}$ vibronic transitions are at 456,489 and $526 \mathrm{~nm}$ respectively. There is no change in the peak positions even at very low concentrations. Hence. in the non-aqueous solvents used here, the $\pi$-system in this block copolymer does not self assemble. The fluorescent spectra (figure 5.1b) of PS-PTCDI-PS in different solvents are similar with a slight shift and mirror image of their absorption spectra. Upon excitation at $366 \mathrm{~nm}$, these solutions show intense fluorescence (figure $5.1 \mathrm{~b}$ inset), with the color being either greenish yellow or yellowish green. 


\section{$0.15 \quad a$}

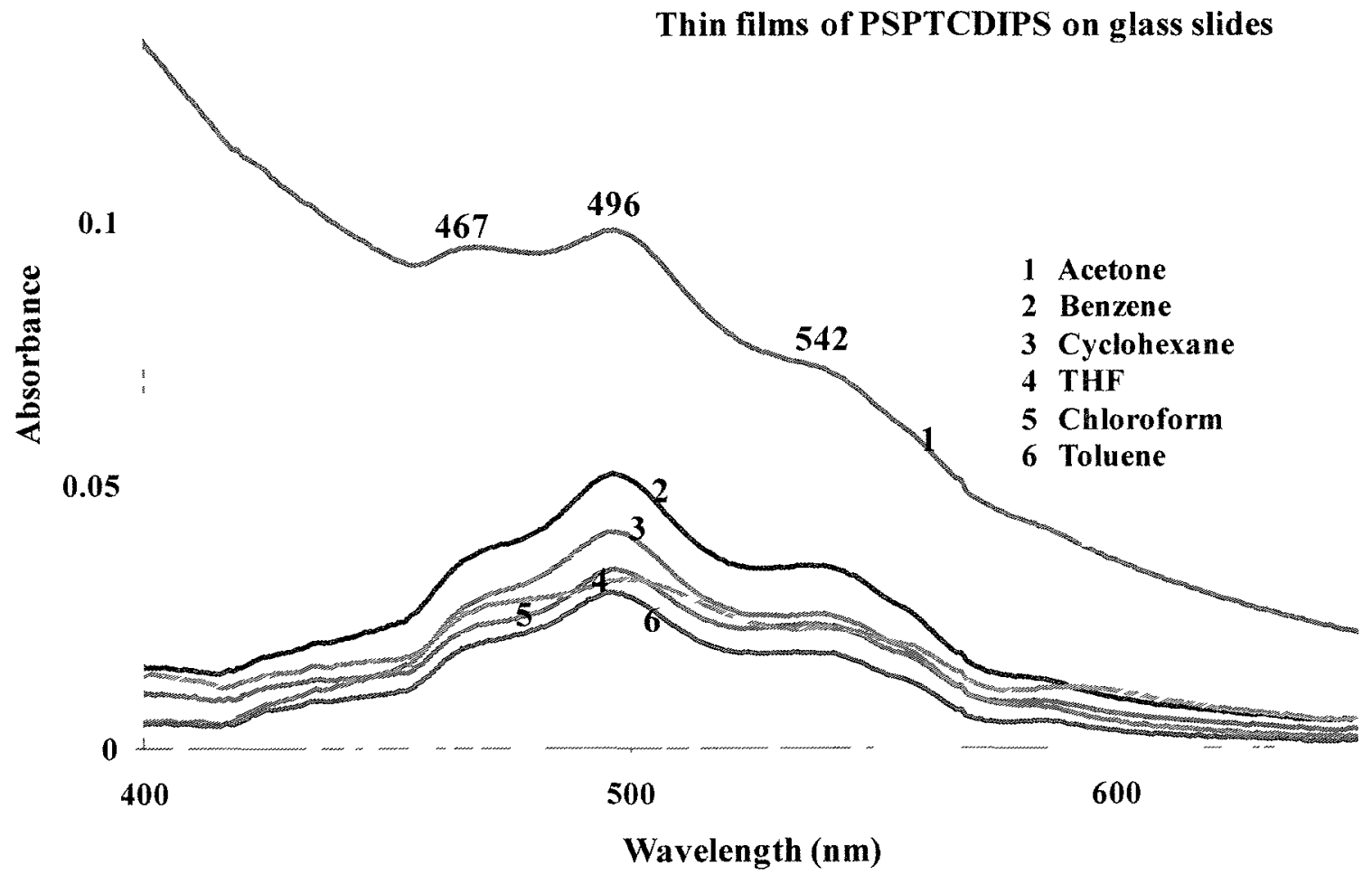

Figures continued in the next page 

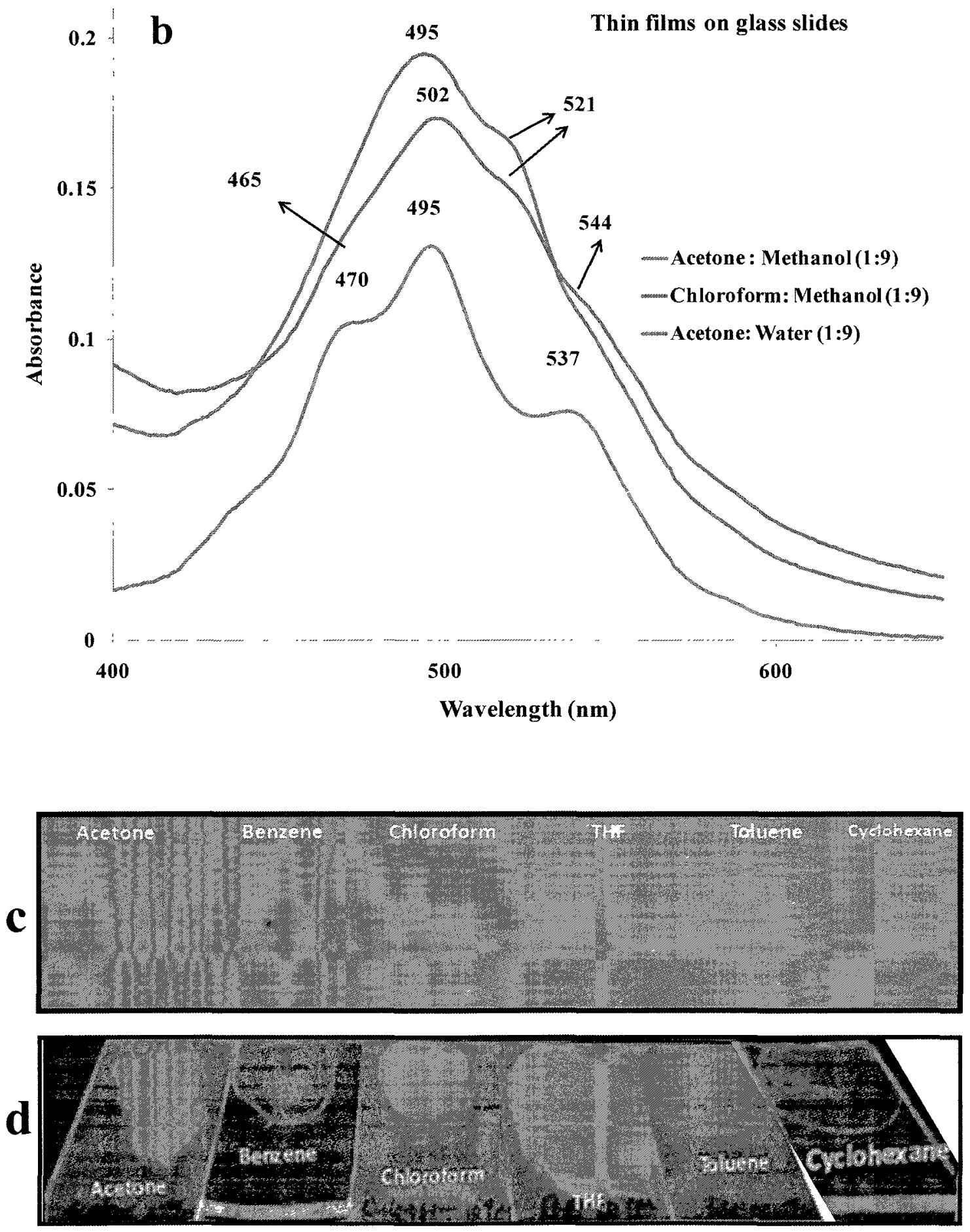

Figure 5.3: (a) UV-Vis spectra of PS-PTCDI-PS drop cast and dried on glass slides from different solvents at $10^{-4} \mathrm{M}$. (b) Absorption spectra of such films from mixtures of solvents. (c) Photographs of the glass slides with PSPTCDIPS from different solvents without excitation and (d) excited at $366 \mathrm{~nm}$. 
Although no self-assembly of PTCDI was seen in solutions, the absorption spectra of the drop cast films (of PS-PTCDI-PS dried from solutions of different solvents) show a different pattern and indicate self-assembly (figure 5.3a). It appears that during the slow solvent evaporation process PS-PTCDI-PS self assembled into an aggregate structure. An intensity reversal is seen (compared to its monomeric form in figures 5.1 and 5.2) between the $S_{0-0}$ and $S_{0-1}$ transition peaks. Comparing the UV spectra of non-aggregated solution and aggregated solid state films, it is seen that the most intense peak of the monomeric state in different solvents shows a bathochromic shift to $542 \mathrm{~nm}$ in the solid state and the $\mathrm{S}_{0-1}$ transition peak is also shifted to $496 \mathrm{~nm}$ and becomes the most intense peak. The absorption peak for $\mathrm{S}_{0-2}$ transition moves to $467 \mathrm{~nm}$ and becomes a shoulder. These spectral features confirm the self-assembly of PS-PTCDI-PS in solid state. , $3,8,25-27^{-27}$ The shift of all the peaks (for $\mathrm{S}_{0-0}, \mathrm{~S}_{0-1}$, or $\mathrm{S}_{0-2}$ transitions) towards the longer wave length indicates the mode of stacking to be J-type. ${ }^{4.8}$ Due to the presence of the short chain atactic PS chain, complete face to face overlapping between two successive perylene units is distorted and a transverse stacking becomes stable. The wide shift of $\mathrm{S}_{0-0}$ transition peak from 526 to $542 \mathrm{~nm}$ confirms the strong stacking as J-type. An interesting observation is that the peak positions remain the same at 542, 496 and $467 \mathrm{~nm}$ for these solid films irrespective of the solvent that these were deposited from, whereas in the corresponding solutions a shift was observed due to different polarities of these solvents. Figure $5.2 \mathrm{~b}$ shows the absorption pattern of drop cast films of PS-PTCDI-PS from mixture of solvents revealing their presence as aggregated forms, with the $S_{0-1}$ peak being more intense. In addition, both $\mathrm{S}_{0-1}$ and $\mathrm{S}_{0-0}$ transition peaks are red shifted. Upon exciting the films deposited on the glass slide with $366 \mathrm{~nm}$ we find that their fluorescent color is 
orange, red or maroon (figure 5.3d) and their fluorescence intensity is different which indicate that the morphologies are different. In the solution all of them were present in monomeric form and showed green fluorescence (figure 5.1) which changes to red or maroon in the solid state which confirms that they are self assembled.

The entire research area on tuning the morphology of amphiphilic diblock polymers depends on the fact that self-assembly of one of the blocks can be achieved by using a solvent for the other block. However, in the present case, we find that in the media which are solvents for the polystyrene segment, the PTCDI did not self-assemble. We believe that a possible reason for this behavior could be that in the preferred solvent, the polystyrene segment is extended (stretched out) and the phenyl groups are in a suitable disposition to interact with the PTCDI segment of a neighboring molecule via aromatic $\mathrm{C}-\mathrm{H}^{-} \pi$ interaction, as illustrated in figure 5.4. Such an interaction would interfere with the self-assembly and stacking of the PTCDI segments in these solvents. While such a $\mathrm{C}-\mathrm{H}^{\cdots}$ interaction generally promotes self-assembly and plays a role in host-guest complexes ${ }^{30}$ in the present case, the opposite effect is seen. We offer this as a possible explanation, but are unable to verify it with techniques such as NMR or IR due to the difficulty in detecting the frequency shift of one proton that would be involved in such an interaction. During the drying of the drop cast films, the phase separation and collapse of the polystyrene segment leads to the aggregation of PTCDI, as shown in figure 5.4. 

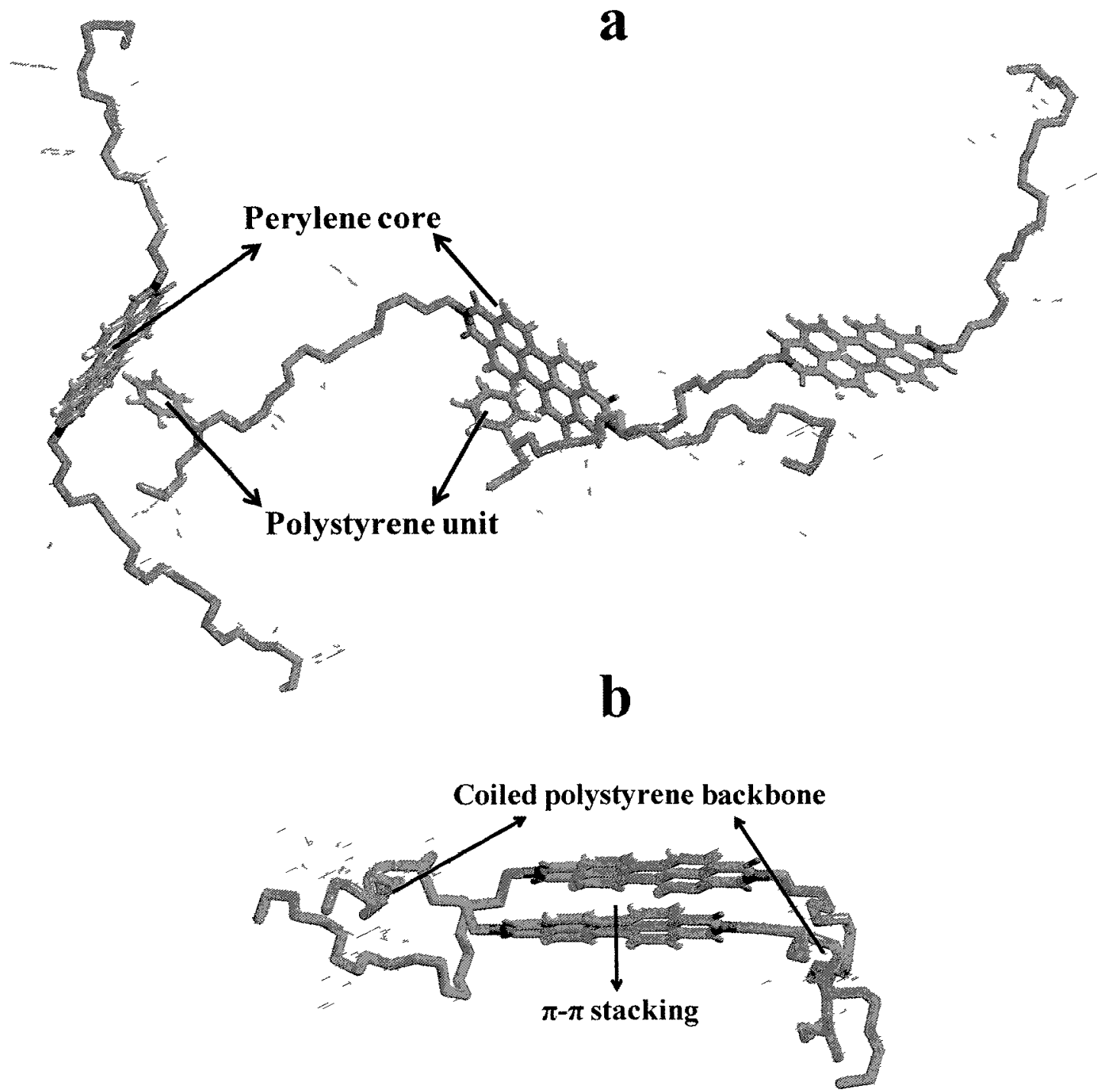

Figures 5.4: Proposed model of (a) $\mathrm{C}-\mathrm{H} \quad \pi$ interaction between a phenyl group of a polystyrene segment and the PTCDI of an adjacent molecule in a good solvent for polystyrene. (b) $\pi$-stacking between neighboring PTCDI segments when the polystyrene segment is coiled (collapsed). 


\subsubsection{Effect of Polarity on Self-assembly}

To qualitatively examine the effect of the polarity and solvent-nonsolvent mixtures on the self-assembly of PS-PTCDI-PS in solution, we recorded the absorption spectra of PS-PTCDI-PS $\left(10^{-5} \mathrm{M}\right)$ in solvent mixtures such as chloroform/ hexane, chloroform/ methanol, acetone/ water and acetone/ methanol. Figure 5.5 shows the absorption and fluorescent spectra for the case of chloroform/ hexane mixtures. Here chloroform is a solvent for PS and hexane is a non-solvent and is non-polar. Apart from a slight shift in peak positions due to change in polarity, there is no change in the spectral pattern from that of the non-aggregate form. The fluorescent spectra (figure 5.5b) are mirror images of the absorption spectra and intensity is almost same for all compositions of chloroform-hexane mixture.

Figure 5.6 shows the absorption and fluorescent spectra of PS-PTCDI-PS in chloroform/ methanol mixtures. Here again chloroform is a good solvent for PS and methanol is a non-solvent for both PS and PTCDI. As the chloroform/ methanol ratio is changed from $9 / 1$ to $1 / 9$, an intensity reversal (figure 5.6a) occurs with higher methanol content (1/9), indicating self-assembly. With this methanol concentration, the $\mathrm{S}_{0-0}$ transition peak drops in intensity, $\mathrm{S}_{0-1}$ peak increases in intensity and the $\mathrm{S}_{0-2}$ peak becomes a shoulder with the whole absorption pattern becoming almost shapeless. A hypsochromic shift from 527 to $519 \mathrm{~nm}$ for the $\lambda_{\max }\left(\mathrm{S}_{0-0}\right)$ is seen along with bathochromic shifts of $S_{0-1}$ and $S_{0-2}$ peaks from 490 to $497 \mathrm{~nm}$ and from 460 to $467 \mathrm{~nm}$ respectively. The excitation of the solutions at $365 \mathrm{~nm}$ gives green fluorescence for all 
compositions except that the solution with chloroform-methanol 1: 9 composition shows non- fluorescent, light maroon color (figure 5.6b).
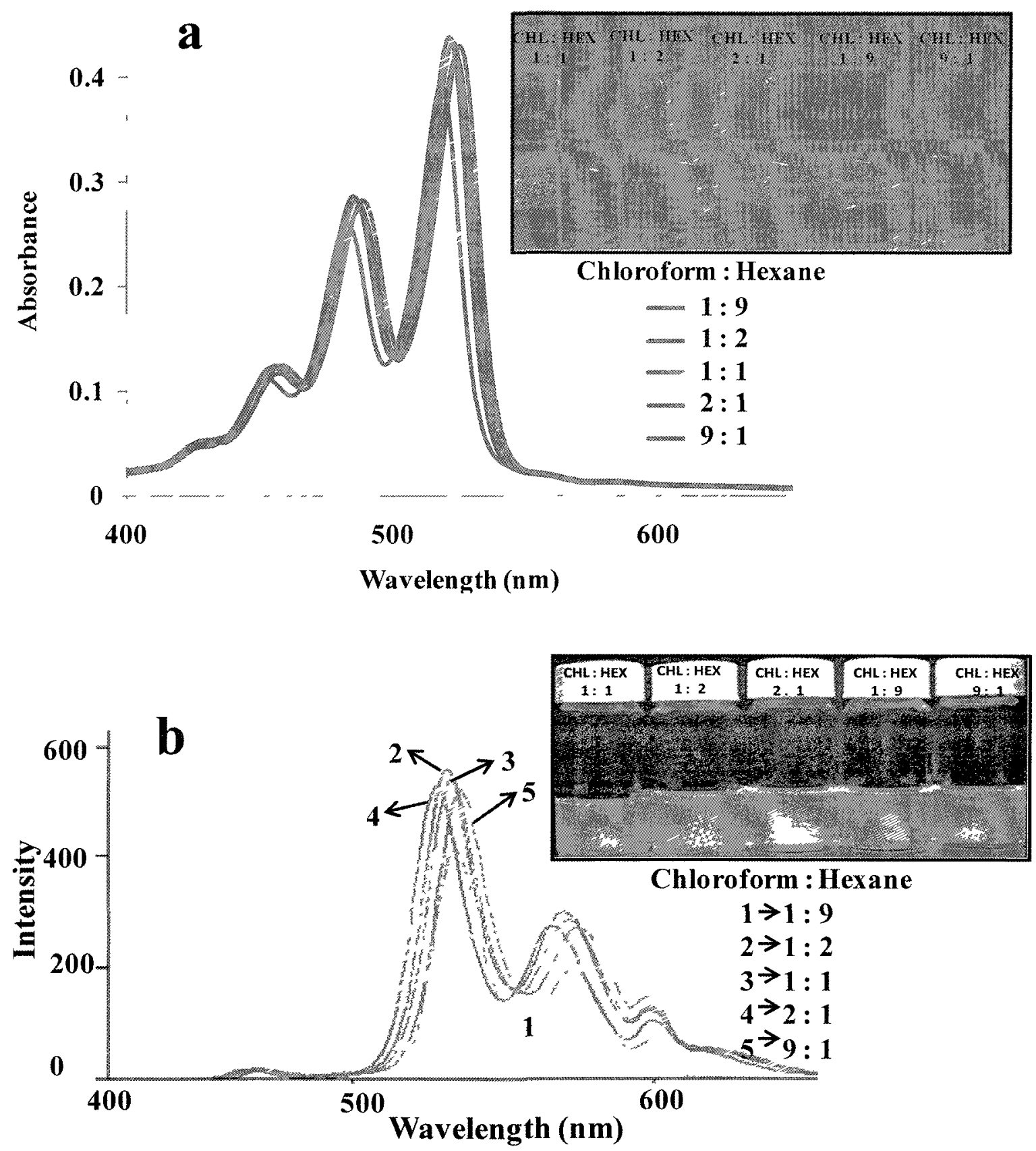

Figure 5.5: (a) UV-Vis and (b) fluorescent spectra of PSPTCDIPS in ChloroformHexane mixtures at $10^{-5} \mathrm{M}$. The inset of both of the figures shows the solutions with different chloroform and hexane ratio without and with excitation at $366 \mathrm{~nm}$. 

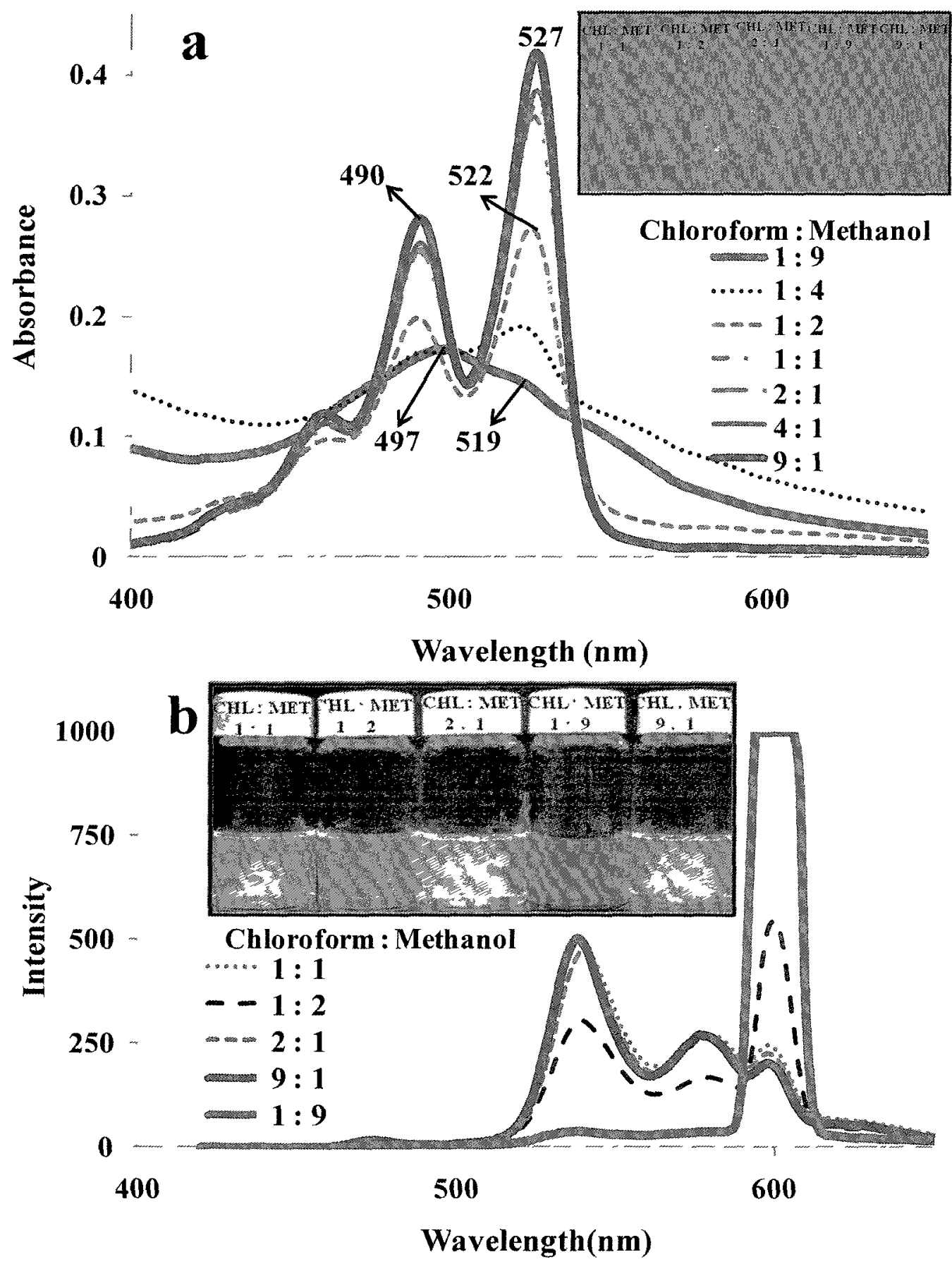

Figure 5.6: (a) UV-Vis and (b) fluorescent spectra of PS-PTCDI-PS in chloroformmethanol mixtures at $10^{-5} \mathrm{M}$. 
The change in intensity and peak positions along with the light maroon color of the solution confirms the self-assembly of PS-PTCDI-PS in the mixture with chloroform/methanol: $1 / 9$ composition. While the molecule exists in monomeric form in chloroform, and it does not dissolve in methanol, self-assembly is seen with only the particular composition as above. Note that as these solutions are made, no precipitation of the solute occurs for a few hours, enabling the recording of the spectra. Thus, it would seem that only with a high content of $90 \%$ methanol, the collapse of the polystyrene segment occurs, leading to aggregation and self-assembly of the PTCDI segment. Thus, the collapse of the polystyrene segment is required for precluding the $\mathrm{C}-\mathrm{H}^{*} \pi$ interaction between a phenyl group of a polystyrene segment and the PTCDI of an adjacent molecule, and enable the self-assembly of the PTCDI segment.

In the case of acetone/ water system, the former is a solvent for PS and neither is a solvent for the PTCDI segment. The absorption spectra in figure 5.7a show the presence of monomeric form only for acetone/water: $9 / 1$ composition. With all other compositions intensity reversal and bathochromic shift of all the peaks are seen, confirming the selfassembly of PTCDI. The $S_{0-0}$ peak shifts from 521 to $538 \mathrm{~nm}$, the $S_{0-1}$ from 486 to $495 \mathrm{~nm}$ and $S_{0-2}$ from 467 to $470 \mathrm{~nm}$. After irradiation with $366 \mathrm{~nm}$ only acetone/water: 9/1 composition shows green fluorescence (figure 5.7c) and all the other compositions show maroon fluorescence confirming its aggregated structure. The acetone-methanol system behaves similar to the chloroform-methanol mixtures. Figure 5.8 shows the presence of aggregate structure only for acetone/methanol: $1 / 9$ composition. These observations with different binary mixtures suggest that control over aggregation and de-aggregation can be 
achieved by small differences in the relative compositions of these mixtures. Such aggregation of PTCDI occurs only when the polystyrene segment is in a collapsed conformation and does not entail the $\mathrm{C}-\mathrm{H} \quad \pi$ interaction.
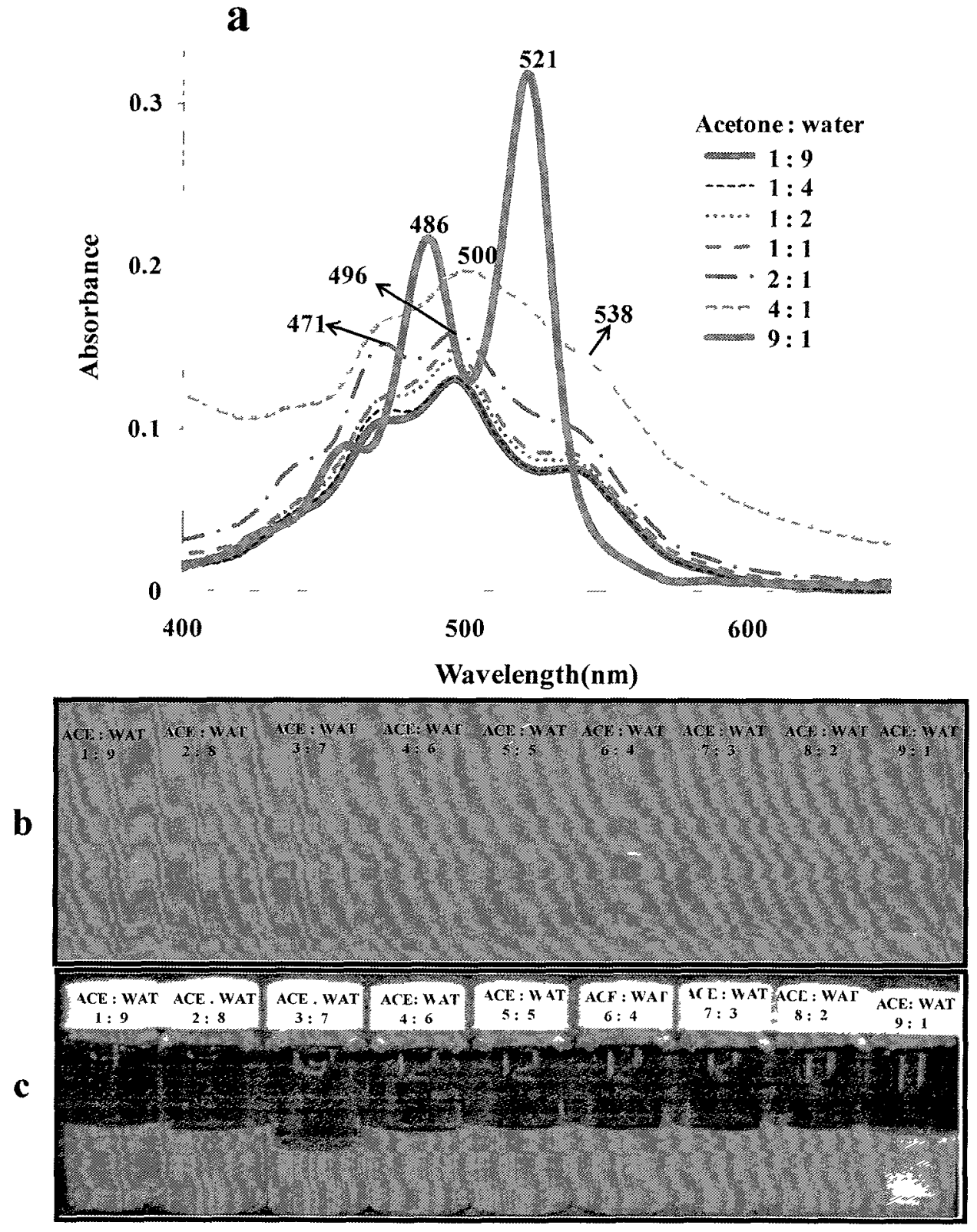

Figure 5.7 (a) UV-Vis spectra of PS-PTCDI-PS in Acetone-water mixtures at $10^{-5} \mathrm{M}$ Photographs of solutions (b) before and (c) after excitation, respectively. 


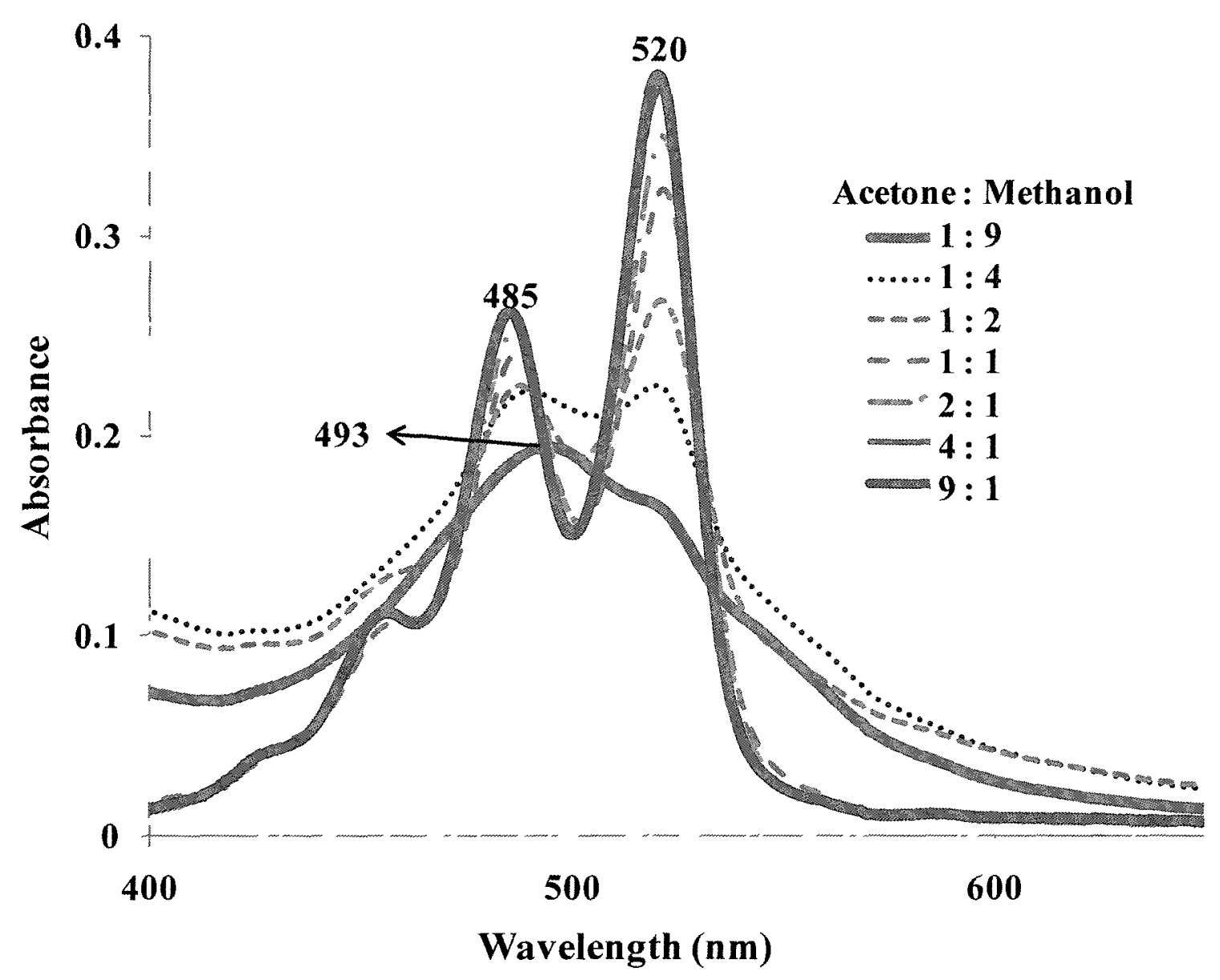

Figure 5.8 UV-Vis spectra of PS-PTCDI-PS in acetone-methanol mixtures $\left(10^{-5} \mathrm{M}\right)$.

\subsubsection{Effect of Temperature}

To study the thermal stability of the aggregates in different binary mixtures we recorded UV-Vis spectra with increasing temperature with a fixed composition in which 
aggregates formed. Figure 5.9a shows the change in the absorption spectra with temperature for PS-PTCDI-PS in chloroform/methanol: $1 / 9$ solution $\left(10^{-5} \mathrm{M}\right)$. The somewhat shapeless spectral pattern develops into well defined peaks with an increase in temperature. At lower temperatures of up to $50{ }^{\circ} \mathrm{C}$ the peak at $550 \mathrm{~nm}$ (bathochromic shifted $\mathrm{S}_{0-0}$ transition peak due to aggregate formation) decreases in intensity and the most intense peak at $505 \mathrm{~nm}$ shows a hypsochromic shift. The peak at $550 \mathrm{~nm}$ vanishes at $60^{\circ} \mathrm{C}$. The appearance of a peak at $530 \mathrm{~nm}$ and the hypsochromic shift of the $505 \mathrm{~nm}$ peak to $494 \mathrm{~nm}$ indicate the breakdown of the aggregated structure to monomers at $60{ }^{\circ} \mathrm{C}$. A different temperature response of absorption spectra is seen in figure $5.9 \mathrm{~b}$ with acetone/water: $1 / 9$ mixture. The typical aggregate absorption pattern persists even at 80 ${ }^{\circ} \mathrm{C}$ with a small hyperchromic shift with temperature. Both $\mathrm{S}_{0-0}$ and $\mathrm{S}_{0-1}$ transition peaks show hypsochromic shift of $8 \mathrm{~nm}$ and $4 \mathrm{~nm}$ respectively. 

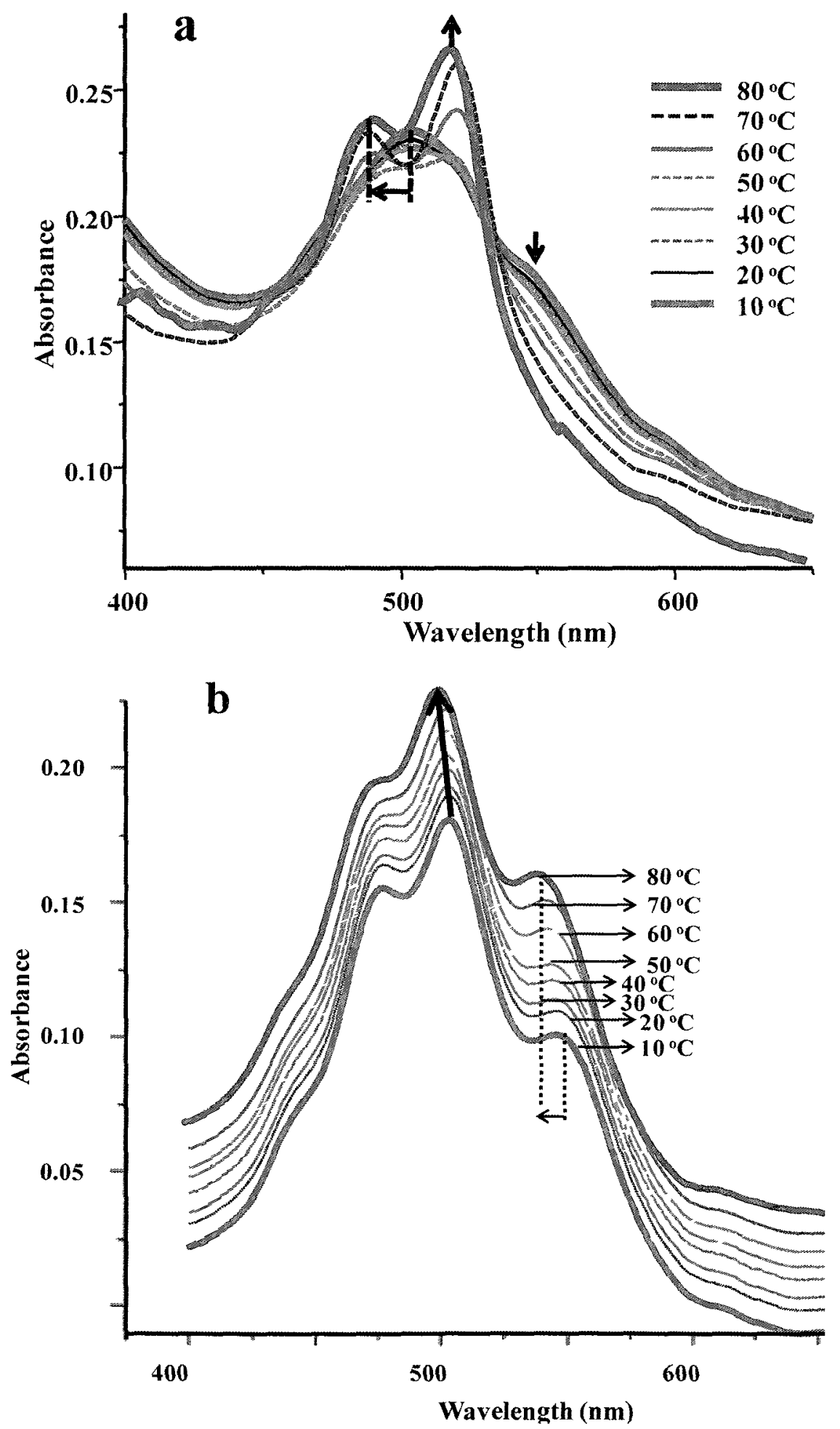

Figure 5.9 Temperature dependent UV-Vis spectra of PS-PTCDI-PS in chloroform/methanol: $1 / 9$ and acetone/water: $1 / 9$ systems $\left(10^{-5} \mathrm{M}\right)$. 


\subsubsection{Morphology by Optical and Scanning Electron Microscopy}

Optical (OM) and scanning electron micrographs (SEM) were recorded for drop cast films from various solvents and mixed solvents. Although PS-PTCDI-PS does not self assemble in any single solvent, it does so during the evaporation of the solvent in drop cast films (see also figure 5.3). Figure 5.10 shows the OM of the films from acetone, chloroform, and tetrahydrofuran (THF). Those from THF appear to be submicron crystalline aggregates, the film from acetone shows spherical assemblies and with chloroform, a web morphology is seen. Figures 5.11a show the SEM of the film from acetone, which consist of nano vesicles ranging in size from 150 to $600 \mathrm{~nm}$, with an average of about $400 \mathrm{~nm}$. An illustration of the formation of such vesicles is shown in figure 5.12, which involves the stacking of the perylene unit of PS-PTCDI-PS with the interdigitation of the polystyrene segments. 

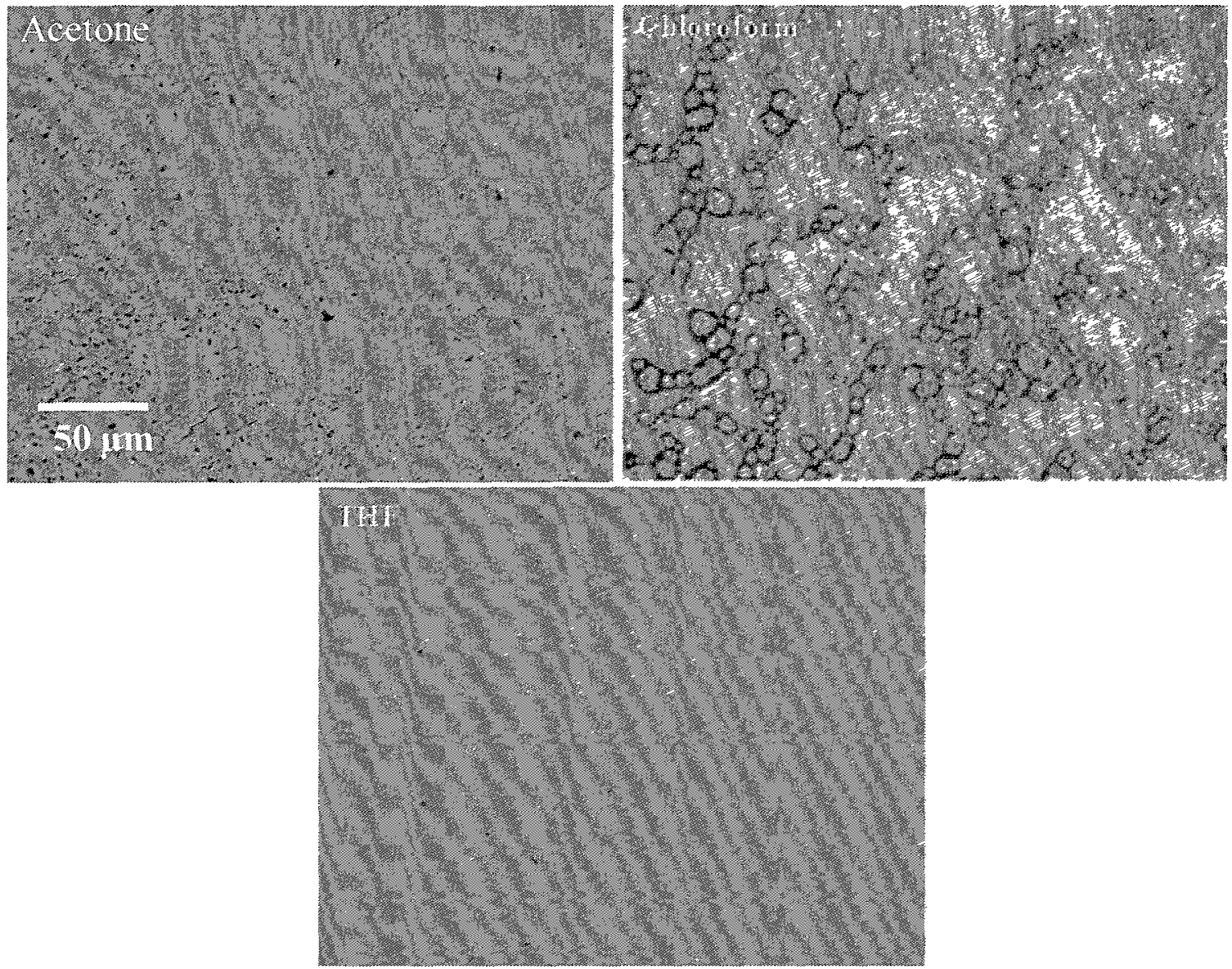

Figure 5.10: Optical micrographs of PS-PTCDI-PS in drop cast films from different solvents. The scale bar is the same for all the images. 

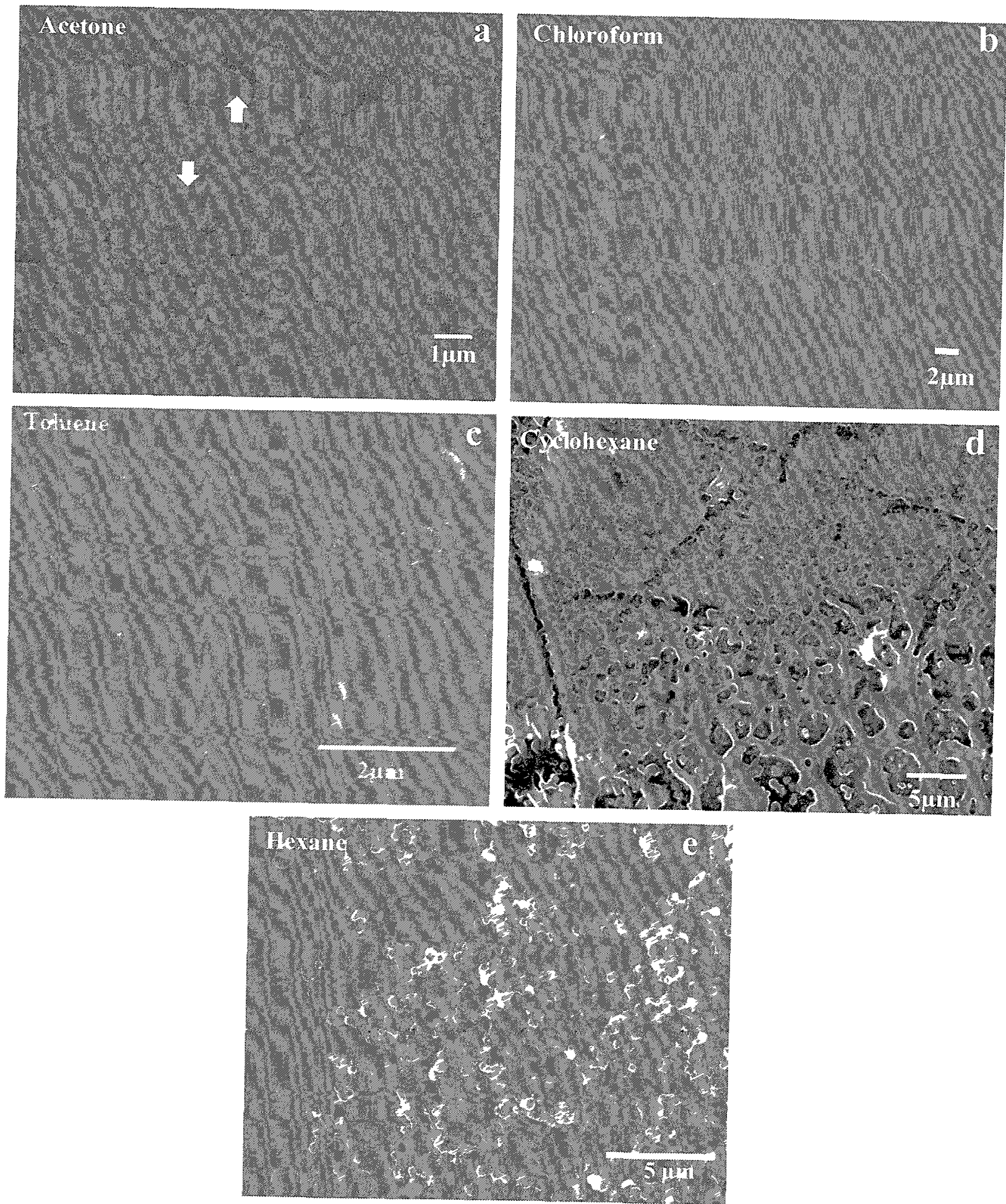

Figure 5.11: SEM micrographs of PS-PTCDI-PS drop cast films from different solvents. $(\mathrm{a}, \mathrm{b})$ : acetone; $(\mathrm{c}, \mathrm{d})$ : chloroform; $(\mathrm{e}, \mathrm{f})$ : tolune; $(\mathrm{g})$ : cyclohexane; $(\mathrm{h})$ : hexane. 


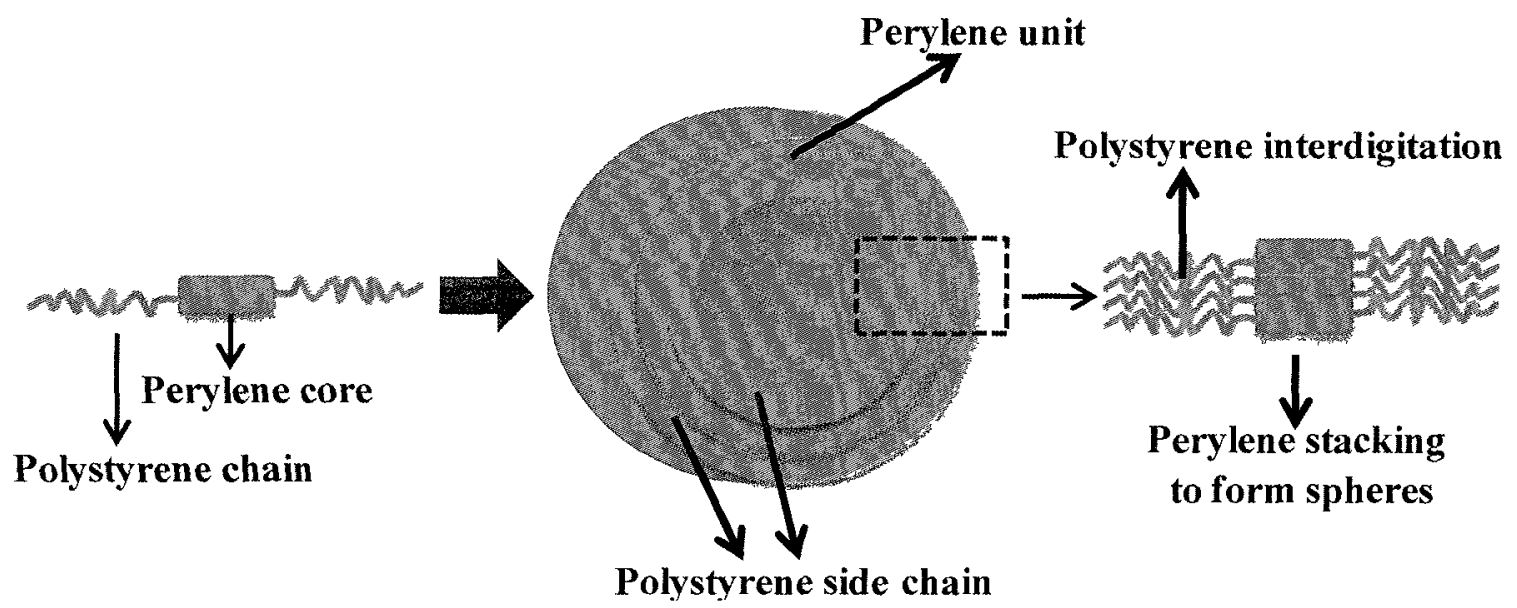

Figure 5.12: Schematic of the formation of vesicles of PS-PTCDI-PS.

With chloroform, a lacy film with web morphology is seen (figures 5.11b), similar to the case of PDMS-PTCDI-PDMS. It was interpreted as due to the stacking of the perylene units and the entanglement of the polymer leading to the wire and web morphology as the film dried (see figure 3 and scheme 2 of ref 37). The morphology of the toluene cast film (figures 5.11c) is similar to that of acetone, but spherical rather than vesicular morphology is seen. Figure 5.11d shows a web morphology is similar to the case of chloroform. Finally, crystalline precipitates are seen with hexane as the solvent (Figure 5.11e) similar to the case of PDMS-PTCDI-PDMS. The crystalline morphology is due to the aggregation of PTCDI. Figure 5.13 shows the SEM of drop cast films using chloroform/hexane and THF/water mixtures. It is seen that while with hexane as the major component, only a precipitate is seen, and addition of a small amount of chloroform (chloroform/hexane: 1/9) results in the formation of large vesicles. On the other hand, spherical morphology is seen for a THF/water composition of $5 / 5$ and $9 / 1$ 
compositions. Thus, irrespective of the solvent composition (except hexane), spherical or vesicular morphology is seen.
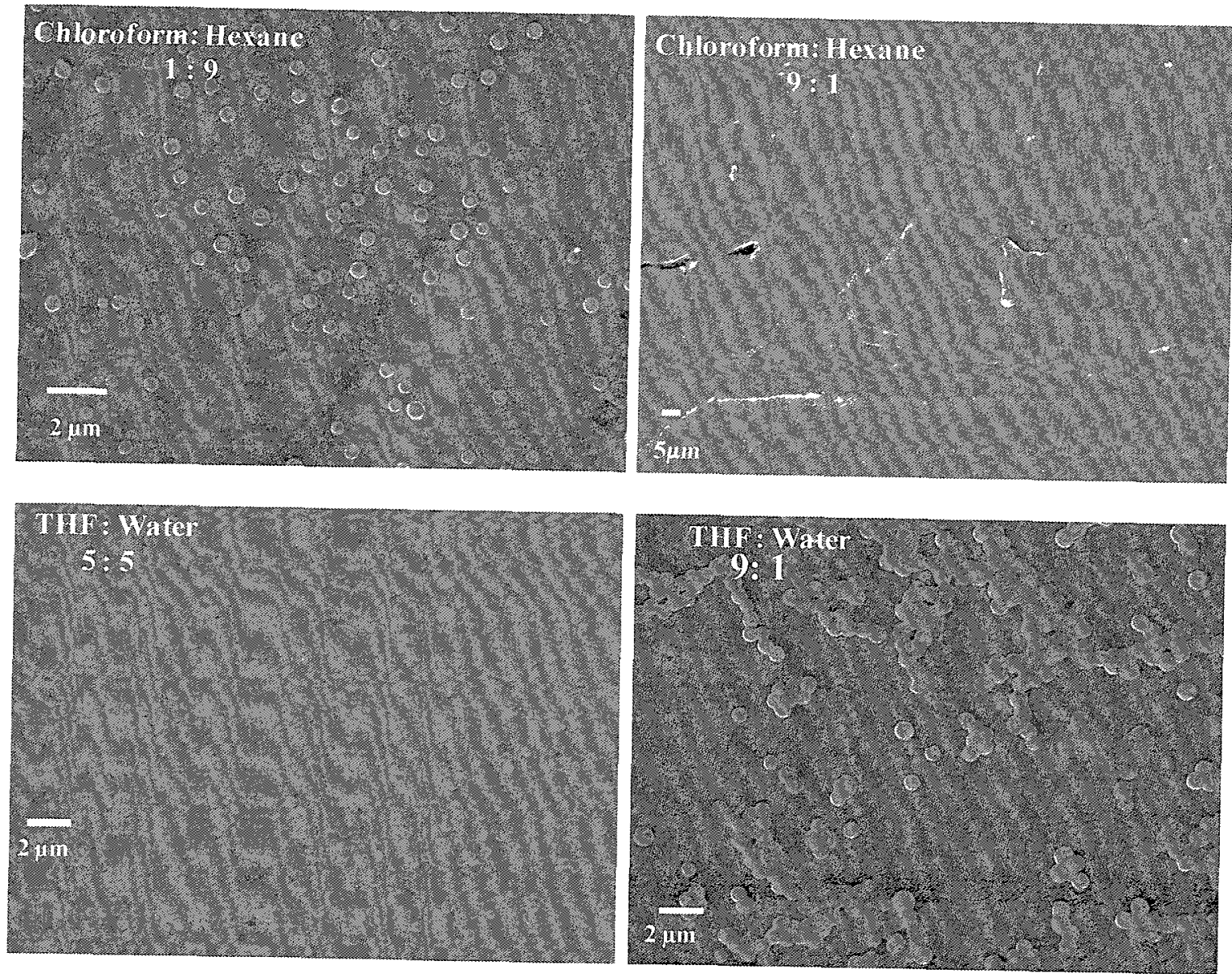

Figure 5.13: SEM micrographs of PS-PTCDI-PS drop cast films from chloroform/hexane and $\mathrm{THF} /$ water mixtures. 


\subsubsection{X-Ray Analysis}

The X-ray diffraction traces of PTDA and PS-PTCDI-PS are given in figure 5.14.

It is seen that the peak positions are different for the two, indicating that the crystalline structure of PTDA is modified by the polystyrene jacket. PS-PTCDI-PS shows diffraction peaks at $8.03,6.34,3.36$ and $3.05 \AA$, confirming the aggregation of the perylene segment in bulk.

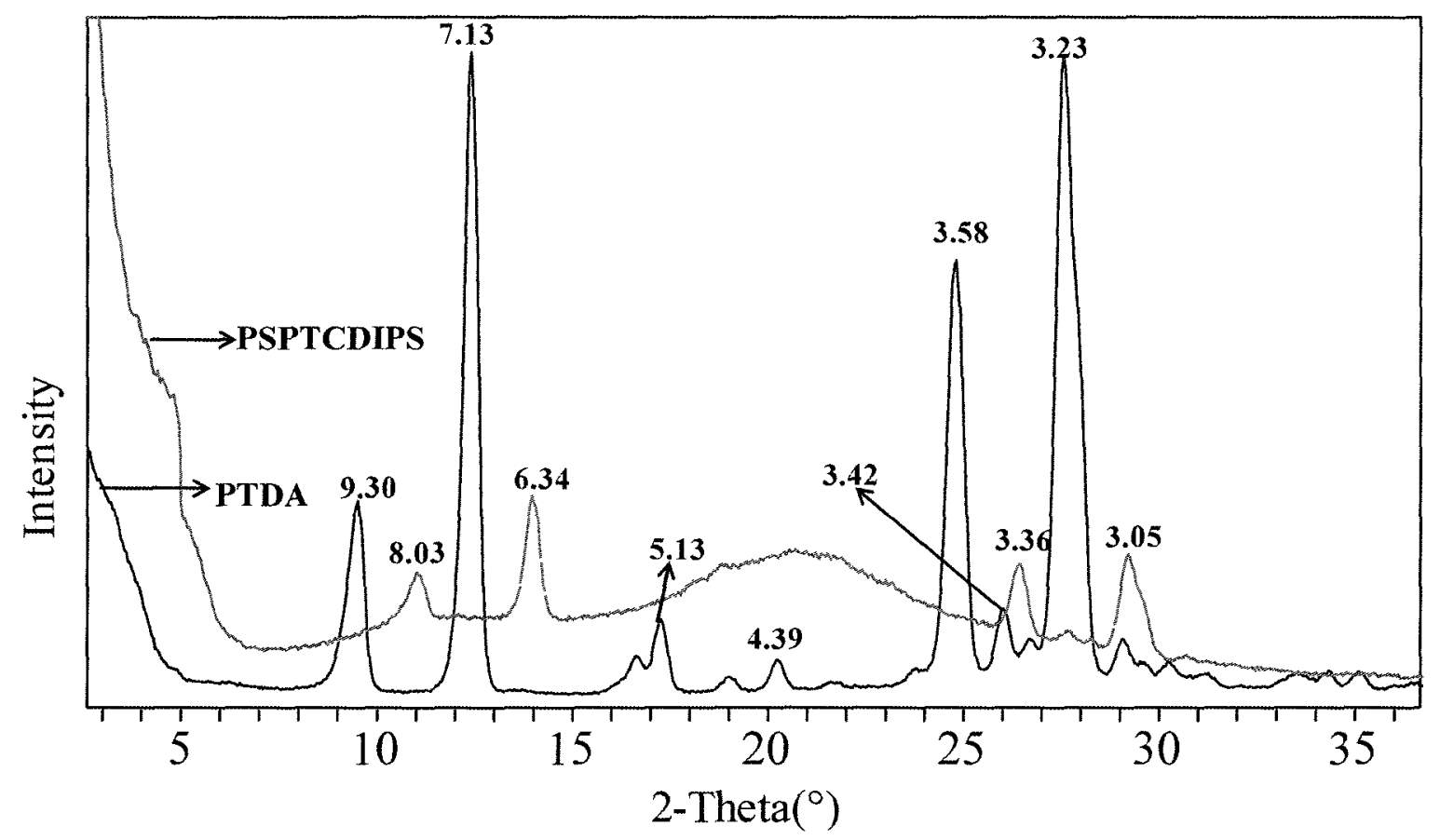

Figure 5.14: X-ray diffraction traces of PS-PTCDI-PS and PTDA. 


\subsection{Conclusions}

Normally, it is expected that a good solvent for the polystyrene segment, which is also a non-solvent for the PTCDI is expected to promote the self-assembly of the latter. However, we find that with PTCDI substituted on both sides with polystyrene, PSPTCDI-PS does not show self-assembly of the perylene segment in any of the solvents that dissolve polystyrene. We attribute this behavior to the $\mathrm{C}-\mathrm{H}^{\cdots} \pi \pi$ interaction between the phenyl groups of the polystyrene segment and PTCDI, when the polystyrene segment is in an extended conformation (good solvent). Collapsing the polystyrene segment by adding a non-solvent (water or methanol) eliminates the $\mathrm{C}-\mathrm{H}^{\cdots} \cdots$ interaction and enables the stacking of PTCDI segment in neighboring molecules. Stacking is seen in the drop cast films from these solvents due to the collapsed conformation of the polystyrene segment in these films upon evaporation of the solvent.

The PS-PTCDI-PS system exhibits properties very different from those of the DJPTCDI described in Chapter 3. In the case of the latter, the aggregation occurred in water beyond a certain concentration, and was stable over a wide temperature range. It was a case of the Jeffamine being water soluble and the PTCDI, not. The $\mathrm{C}-\mathrm{H}^{\cdots}{ }^{\cdots} \pi$ type of interaction did not exist in that case. The self-assembly could also be modulated by aqueous-non aqueous solvent mixtures. Thus the nature of the polymer used and interactions such as $\mathrm{C}-\mathrm{H}^{\cdots \cdots} \pi$, in addition to the usual hydrogen bonding or $\pi-\pi$ stacking would have an influence on the self-assembly of the chromophores. 


\subsection{References}

1. Hill, J. P.; Jin, W.; Kosaka, A.; Fukushima, T.; Ichihara, H.; Shimomura, T.; Ito, K.; Hashizume, T.; Ishii, N.; Aida, T. Science 2004, 304, 1481.

2. Pisula, W.; Kastler, M.; Wasserfallen, D.; Pakula, T.; Mullen, K. J. Am. Chem. Soc. 2004, 126,8074 .

3. Wurthner, F.; Thalacker, C.; Sautter, A.; Adv. Mater. 1999, 11, 754.

4. Wurthner, F.; Thalacker, C.; Sautter, A.; Schartl, W.; Ibach, W.; Hollricher, O. Chem. Eur. J. 2000, 6, 3871.

5. Wurthner, F. Chem. Comm. 2004. 17, 1564.

6. von Berlepsch, H.; Bottcher, C.; Dahne. L. J. Phys. Chem. B 2000, 107, 8792.

7. Mishra, A.; Behera, R. K.; Behera, P. K.; Mishra, B. K.; Behera, G. B. Chem. Rev. $2000,100,1973$.

8. Wurthner, F.; Yao, S.; Beginn, U. Angew. Chem., Int. Ed. 2003, 42, 3247.

9. Yao, S.; Beginn, U.; Gress, T.; Lysetska, M.; Wurthner, F. J. Am. Chem. Soc. 2004, 126,8336 .

10. Yagai, S.; Ishii, M.; Karatsu, T.; Kitamura, A. Angew.Chem. Int. Ed. 2007, 46, 8005.

11. Ghadiri, M. R.; Granja, J. R.; Milligan, R. A.; McRee. D. E.; Khazanovich, N. Nature $1993,366,324$.

12. Rosenthal-Aizman, K.; Svensson, G.; Unden, A. J. Am. Chem. Soc. 2004, 126, 3372.

13. Hartgerink, J. D.; Beniash, E.; Stupp, S. I. Science 2001, 294, 1684.

14. Niece, K. L.; Hartgerink, J. D.; Donners, J. J. J. M.; Stupp, S. I. J. Am. Chem. Soc. $\mathbf{2 0 0 3}, 125,7146$. 
15. Weitz, R.T.; Amsharov, K.; Zschieschang, U.; Villas, E. B.; Goswami, D.K.; Burghard, M.; Dosch, H.; Jansen, M.; Kern, K.; Klauk, H. J. Am.Chem. Soc., 2008, 130, 4637.

16. Tatemichi, S.; Ichikawa, M.; Koyama, T.; Taniguchi, Y.; Appl. Phys. Lett. 2006, 89, 112108.

17. Malenfant, P. R. L.; Dimitrakopoulos, C. D.; Gelorme, J. D.; Kosbar, L. L.; Graham, T. O. Curioni, A.; Andreoni, W. Appl. Phys. Lett. 2002, 80, 2517.

18. Gundlach, D. J.; Pernstich, K. P.; Wilckens, G.; Gruter, M.; Haas, S.; Batlogg, B. J. Appl. Phys. 2005, 98, 064502.

19. Wang, Y.; Chen, Y.; Li, R.; Wang, S.; Su, W.; Ma, P.; Wasielewski, M. R.; Li, X.; Jiang, J. Langmuir 2007, 23, 5836.

20. Sui, G., Orbulescu, J.; Mabrouki, M.; Leblanc, R.M.; Liu, S.; Gregg. B.A. Chem phys Chem 2002, 3, 1041.

21. Chen, Z.; Baumeister,U.; Tschierske, C.; Wurthner, F. Chem. Eur. J. 2007, 13, 450.

22. Yao, D.; Bender, T. P.; Gerroir, P. J. Sundararajan, P. R. Macromolecules 2005, 38, 6972.

23. Yao, D.; Tuteja, B.; Sundararajan, P. R. Macromolecules 2006, 39, 7786.

24. Balakrishnan, K.; Datar, A.; Naddo, T.; Huang, J.; Oitker, R.; Yen, M.; Zhao, J.; Zang, L. J. Am. Chem. Soc. 2006, 128, 7390.

25. Balakrishnan, K.; Datar, A.; Oitker, R.; Chen, H.; Zuo, J.; Zang, L.; J. Am. Chem. Soc. 2005, 127, 10496.

26. Datar, A.; Balakrishnan, K.; Yang, X.; Zuo, X.; Huang, J.; Oitker, R.; Yen, M.; Zhao, J.; Tiede, D. M.; Zang, L. J. Phys. Chem. B 2006, 110, 12327. 
27. Datar, A.; Oitker, R.; Zang, L.; Chem. Commun. 2006, 15, 1649.

28. He, X.; Liu, H.; Wang, N.; Ai, X.; Wang, S.; Li, Y.; Huang, C.; Cui, S.; Li, Y.; Zhu, D. Macromol. Rapid Commun. 2005, 26, 721 .

29. Baby, J.; Asha, S. K. Chem. Mater. 2008, 20, 169.

30. (a) Takahashi, H.; Tsuboyama, S.; Umezawa, Y.; Honda, K.; Nishio, M. Tetrahedron 2000, 56, 6185. (b) Barooah, N.; Baruah, J. B. J. Molec. Struc. 2008, 872, 205. 


\section{Chapter VI}

Tubular or Sub-Surface Morphology of a Substituted Phthalocyanine upon Self-assembly in Polymer Matrices: Effect of the Casting Solvent.

Chem. Eur. J. 2011(in press). 


\subsection{Introduction}

Self-assembly and molecular recognition are ubiquitous in Nature. Although most chemical species associate and crystallize, the self-assembly is characterized by spontaneity and reversibility and facilitated by one or more of non-covalent forces such as hydrogen bond, aromatic, columbic or hydrophobic interactions. ${ }^{1-5}$ Mimicking the biological process of selection and assembly, synthetic analogues have been designed to self assemble in a precise manner and these have been targeted for potential applications in functional devices. Lehn ${ }^{3}$ noted that such supramolecular design is essentially the science of informed matter, deriving from the principles of chemistry, physics and biology. Monomers bearing self-complimentary DAAD-ADDA donor-acceptor complexes have been used for designing supramolecular "transient" polymers and copolymers. ${ }^{3}$ Similar to folding of proteins, foldable polymers with associating aromatic chromophores have also been designed. Self-assembled specific shapes have also been used as hosts as "molecular flasks". 6

Morphology of materials in confined space is another active area of research currently. Manipulations of reactions that are "confined" in the molecular flasks mentioned above have been reviewed. ${ }^{6}$ Thin films of polymers and block copolymers are considered confined, as they are restricted to two dimensions, the third (thickness) being of the same order as the coil dimensions. ${ }^{7-13}$ Studies have been reported on crystallization in ultra thin polymer films ${ }^{7}$ and in small nanodomains. ${ }^{8}$ Polymer diffusion ${ }^{9}$ and dynamics ${ }^{10}$ under confinement, conformation and molecular mobility of polymers confined in nanochannels ${ }^{11}$ (e.g., in urea), etc. have also been studied. 
Another emerging area is the study of the self-assembly process and features when a molecule or part of the molecule bearing the self-assembly code is confined (restricted) by physical or chemical means. Self-assembly triggered by spontaneous phase separation from a polymer or other matrices is an area of interest. for fabricating devices in which functional molecules have to be dispersed in a matrix. ${ }^{14}$ Normally, when small molecules phase separate from polymer matrices, they would diffuse to the surface of the film. aggregate and crystallize. ${ }^{15}$ However, the phase separated morphology of self assembling small molecules when confined in a polymer matrix would be different. We found that in the case of hydrogen bond mediated self-assembly of mono and biscarbamates in a polycarbonate matrix, the phase separation and aggregation occur even before the small molecule diffused to the surface. ${ }^{16}$ As a result, the crystalline aggregate of these molecules are found in the bulk of the polymer film and not on the surface. Organized domains of colloidal size were seen. Another type (chemical confinement) involves attaching a polymer to a self-assembling aromatic chromophore. These are macromolecular surfactants, in which the head group is solvophobic. In this regard, we reported the self-assembly of perylene diimide with PDMS chain attached to one end as well as both ends. ${ }^{17}$ Vesicular and nano-web morphologies were seen, respectively, in these two cases.

The existing and potential applications of organic functional molecules in electronic $^{1}$ and light emitting devices ${ }^{2}$ require an understanding of the self-assembly of organic small molecules in polymer matrices. The phase separation and association 
characteristics of these self assembling molecules from polymer matrices are different from the behaviour of other molecules which do not possess the self-assembling code.

Phthalocyanines are a class of typical photoconductor, which exist either metallized or metal-free and exhibit polymorphism of crystal structure. In the case of metal-free phthalocyanine, the X-form is highly photoconductive. ${ }^{18}$ The large aromatic, planar structure of the molecule confers it the attributes of self-assembly. The insolubility in common solvents is circumvented by peripheral substitution, and this leads to nonplanarity and packing arrangements which are different from the unsubstituted form. ${ }^{19-}$ ${ }^{27}$ Crystal structures of both forms are known. Phthalocyanines show intense absorption in the visible and near-IR regions (600-900 nm) and exhibit excellent chemical, light and thermal stability. ${ }^{28} \mathrm{~A}$ substituted $\mathrm{Zn}$-Pc was used for strength enhancement of organogels as well as to make them photoconducting, through in situ cross linking. ${ }^{29}$

In this chapter we discuss the $\pi$-interaction mediated self-assembly of a substituted phthalocyanine in two types of polymers, bisphenol-A polycarbonate (BPAPC) and poly (methyl methacrylate) (PMMA) and the effect of the casting solvent on the morphology. We used $1,4,8,11,15,18,22,25$-octabutoxy-29H, $31 \mathrm{H}-$ phthalocyanine ( $\mathrm{Pc}$ ) (Scheme 6.1), which is soluble and is non-planar. We find that the self-assembled morphology of $\mathrm{Pc}$ depends on the solvent used, and not on the type of polymer. Throughout this paper, we use the term $\mathrm{Pc}$ and phthalocyanine to refer to the substituted phthalocyanine shown in figure 6.1. 


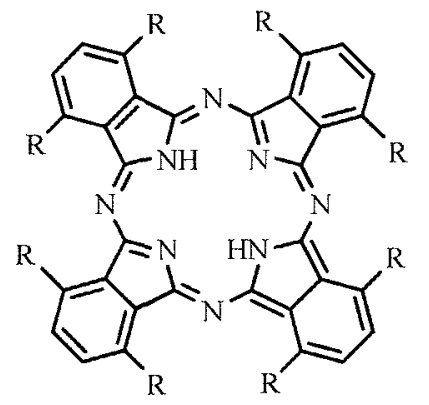

$\mathrm{R}=\mathrm{O} \sim \mathrm{CH}_{3}$<smiles>CC(C)(C)OC(=O)Oc1ccc(C(C)(C)c2ccc(C(C)(C)C)cc2)cc1</smiles>

Polycarbonate (BPAPC)

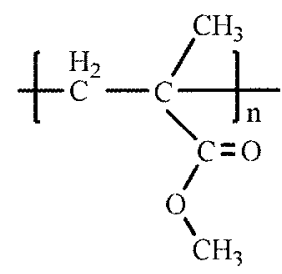

Polymethylmethacrylate(PMMA)

$1,4,8,11,15,18,22,25-$ Octabut oxy -29H,31H-phthalocy anine (Pc)

Figure 6.1: Chemical structure of Pc, BPAPC and PMMA.

\subsection{Experimental:}

Poly (bisphenol-A-carbonate) (BPAPC) $\left(\mathrm{M}_{\mathrm{w}}=64,000\right.$, GPC),1,4,8,11,15,18,22,25-Octabutoxy-29H,31H-phthalocyanine (Pc) and 1,1,2,2tetrachloroethane (TCE) were purchased from Aldrich Chemical Company Inc. and chloroform (Spectro grade) was purchased from Caleadon Industries Ltd. and used without further purification. Poly (methyl methacrylate) (PMMA) $\left(\mathrm{M}_{\mathrm{w}}=350,000\right)$ was purchased from Aldrich. Concentrations of Pc from 0.85 to 5 wt. \% were mixed with the polymers. Films were prepared by dissolving appropriate mixtures of $\mathrm{Pc}$ and BPAPC or PMMA in TCE and $\mathrm{CHCl}_{3}$ respectively (with a solute concentration of $20 \%$ ) and coating on a glass substrate using an electrically driven film coater. Films prepared with chloroform were dried at a very low rate of solvent evaporation at ambient condition for $24 \mathrm{~h}$, and then under vacuum for $48 \mathrm{~h}$ at $50^{\circ} \mathrm{C}$. Films prepared in TCE were initially dried at ambient condition for $24 \mathrm{~h}$, and these were then dried under vacuum at $60^{\circ} \mathrm{C}$ for $48 \mathrm{~h}$. The thickness of the films was about 14-18 $\mu \mathrm{m}$. Thinner films (thickness of 2-6 $\mu \mathrm{m}$ ) were prepared using a spin coater (Spin Coater KW-4B, CHEMAT Technology). In this case, a 
solute concentration of $20 \%$ was used, and with speeds of 500 and 1000 RPM for 30 seconds, the film thickness was 4-6 $\mu \mathrm{m}$ and 2-3 $\mu \mathrm{m}$ respectively. In addition, Pc was dissolved in these two solvents without the polymer, deposited on glass slides and dried by evaporation.

The UV-Vis spectroscopic measurements were performed at ambient conditions using a Varian CARY 3 UV-Vis spectrophotometer. The path length of the quartz sample cube was $10 \mathrm{~mm}$. Fluorescence scans were recorded using a Varian CARY Eclipse Fluorescence Spectrophotometer (Varian Inc., Palo Alto, CA) with a bandwidth of $5 \mathrm{~nm}$ for excitation and $5 \mathrm{~nm}$ for emission. All measurements were performed at room temperature and processed using the software CARY Eclipse WinFLR. The films were attached to the holder by a small magnet and placed in the path of radiation to record the spectra. The absorption spectra were also collected for the solutions of Pc in TCE and chloroform. X-ray diffraction data were collected using a Philips automated powder diffractometer (Model PW 1710) with Ni-filtered $\mathrm{CuK}_{\alpha}$ radiation $(\lambda=1.5412 \AA$ ). The MDI Data Scan 3.2 software (Materials Data Inc., Livermore, CA) was used for data collection and MDI Jade 5.0 software was used for data analysis.

A Zeiss Axioplan 2 imaging universal optical microscope with Q-imaging Retiga digital camera was used to record optical micrographs (OM). The length of the crystals formed was calculated using the "Northern Eclipse version 6.0" image analysis software. A Linkam LTS 350 hot stage with a Linkam TMS 94 controller was used to study the growth of phthalocyanine crystal by itself and in the films with different 
concentrations. The sample in the hot stage was heated at a rate of $5^{\circ} \mathrm{C} / \mathrm{min}$ to a maximum of $250^{\circ} \mathrm{C}$ and cooled at a very slow rate of $1^{\circ} \mathrm{C} / \mathrm{min}$.

In addition, either JEOL JSM-6400 or Tescan Vega-II XMU VPSEM scanning electron microscope (SEM) was used. The samples were fixed by conductive tap onto a glass slide and the surface of the sample was coated with gold/ palladium $(80 / 20 \%)$ alloy layer by a HUMMER VII Sputtering System (ANATECH Ltd, Alexandria, VA.). The thickness of the conductive layer was 9-10 nm. Confocal Laser Scanning Microscopy was performed in air by a Zeiss LSM510 (35\% laser intensity) with a Plan-Acochromat $63 \mathrm{x} / 1.4$ Oil Disc objective. The samples were excited at $405 \mathrm{~nm}$ and images were collected at $410 \mathrm{~nm}$.

${ }^{1} \mathrm{H}$ and ${ }^{13} \mathrm{C}$ NMR spectra were recorded at room temperature on a Bruker-400 spectrometer either in chloroform or TCE as the solvent and tetramethyl silane as the internal standard.

\subsection{Results and Discussion}

We use the following notation to describe the samples: 2Pc/BPAPC/TCE denotes the sample with $2 \%$ (wt) of Pc in BPAPC, prepared with TCE. Likewise, 3Pc/PMMA/CHL denotes a sample with 3\% Pc in PMMA, prepared with chloroform. 


\subsubsection{Microscopy}

\section{Pc/BPAPC/TCE}

Charge transport molecules such as the N.N'-diphenyl-N,N'-bis(3-methylphenyl)[1,1'-biphenyl]-4,4'-diamine (TPD) and tri-p-tolylamine (TTA) are usually dispersed in a polycarbonate matrix in the case of opto-electronic devices. ${ }^{15}$ It is known that homogeneous films of polycarbonate could be prepared with these charge transport molecules with up to $50 \%(\mathrm{wt} / \mathrm{wt})$ concentration. Phase separation would be seen only upon annealing the films at a temperature close to the glass transition $\left(\mathrm{T}_{\mathrm{g}}\right)$ of the polycarbonate. However, a different behaviour is seen with Pc in the BPAPC and PMMA matrices, which is attributed to self-assembly. We point out that although TPD and TTA phase separate and crystallize, it does not occur until the film is annealed at a temperature close to the $\mathrm{T}_{\mathrm{g}}$ of the polycarbonate $\left(150^{\circ} \mathrm{C}\right)$. The phase separated crystal growth is not spontaneous and these small molecules are not considered "self-assembling". They do not possess the common "self-assembly codes" such as hydrogen bonding moieties. The nonplanarity caused by the twisted conformations of the triphenyl segments of TPD and TTA molecules prevent the aromatic $\pi$-stacking. However, such $\pi$-stacking, which is one of the self-assembling codes, is present in the family of phthalocyanine molecules. We realize that the substituted Pc used here is non-planar and the effectiveness of $\pi$-stacking might be reduced. However, the typical $\pi$-stacking between dimers of these phthalocyanines has been observed in their crystal structure (see below).

Figure 6.2 shows the OM of Pc in BPAPC, prepared with TCE. Even with $1 \%$ (wt) concentration of Pc, needle-like crystals form, with their length increasing with Pc 
concentration. With $3 \%$, the crystals are overlapping and the percolation threshold is reached (The percolation threshold is the minimum concentration with which connectivity of the crystals throughout the matrix is achieved). A plot of the average length of the crystals with concentration of $\mathrm{Pc}$ is shown in figure $6.3 \mathrm{a}$ (measurements on 50 crystals were used for calculating the average and the error). It is seen that the increase in length between 3 and $5 \%$ is significant. To confirm that the crystal growth occurs during solvent evaporation, the in situ growth was studied. The solution was spread onto the glass substrate by the electrical coater and progression of crystal formation was followed with time and concentration with OM. The length of crystals with time is shown in figure $6.3 \mathrm{~b}$. It is seen that the maximum length is reached within 20 minutes. The lengths of the crystals measured in the solid films and in the drops of the solutions are similar. Another feature seen in the large crystals in figure $6.2 \mathrm{e}$ is that these are tubular. 

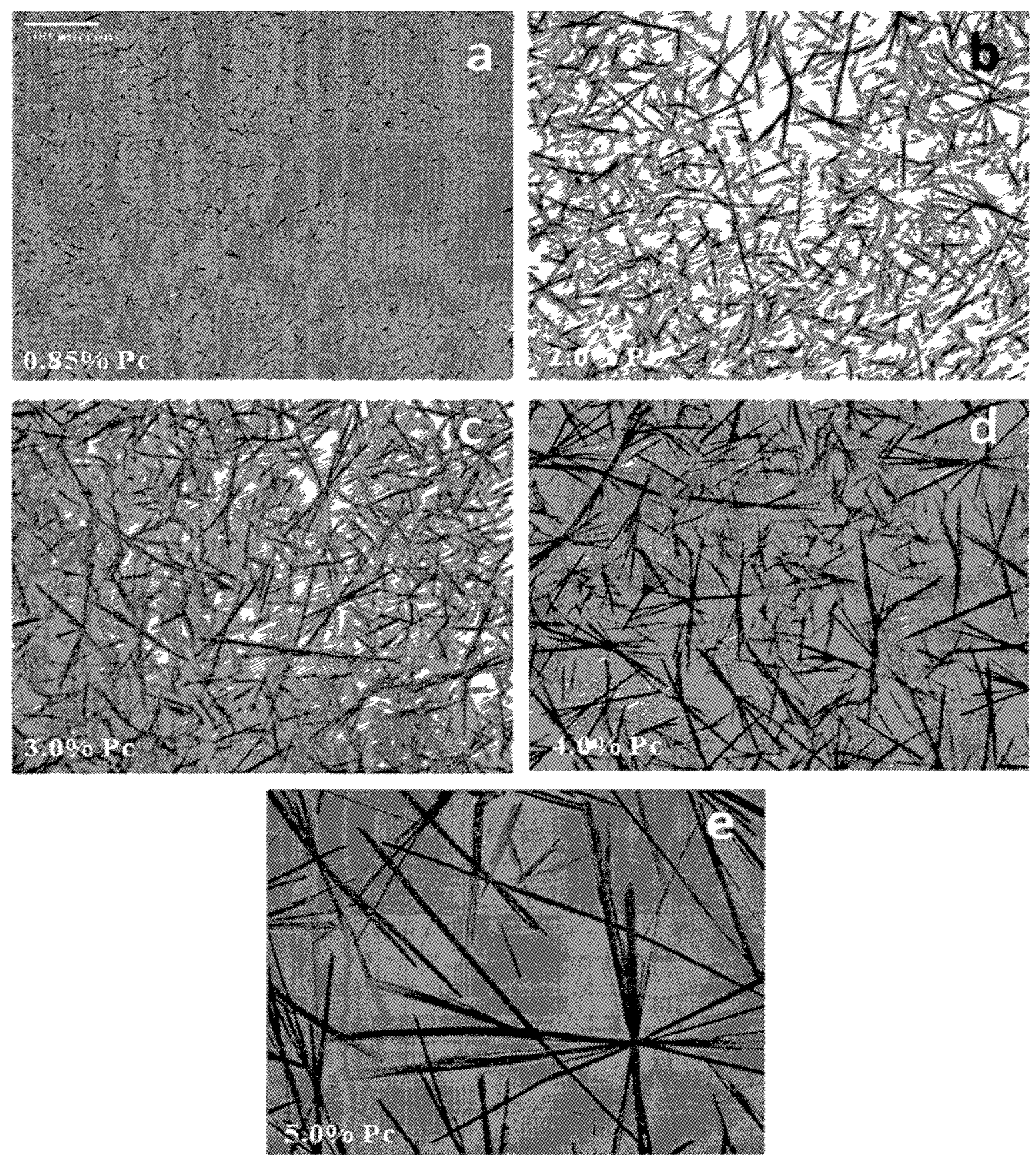

Figure 62 Optical micrographs of Pc/BPAPC/TCE films with (a-e) 0.85 to $5 \%$ concentration of Pc. The scale is the same for (a-e) 

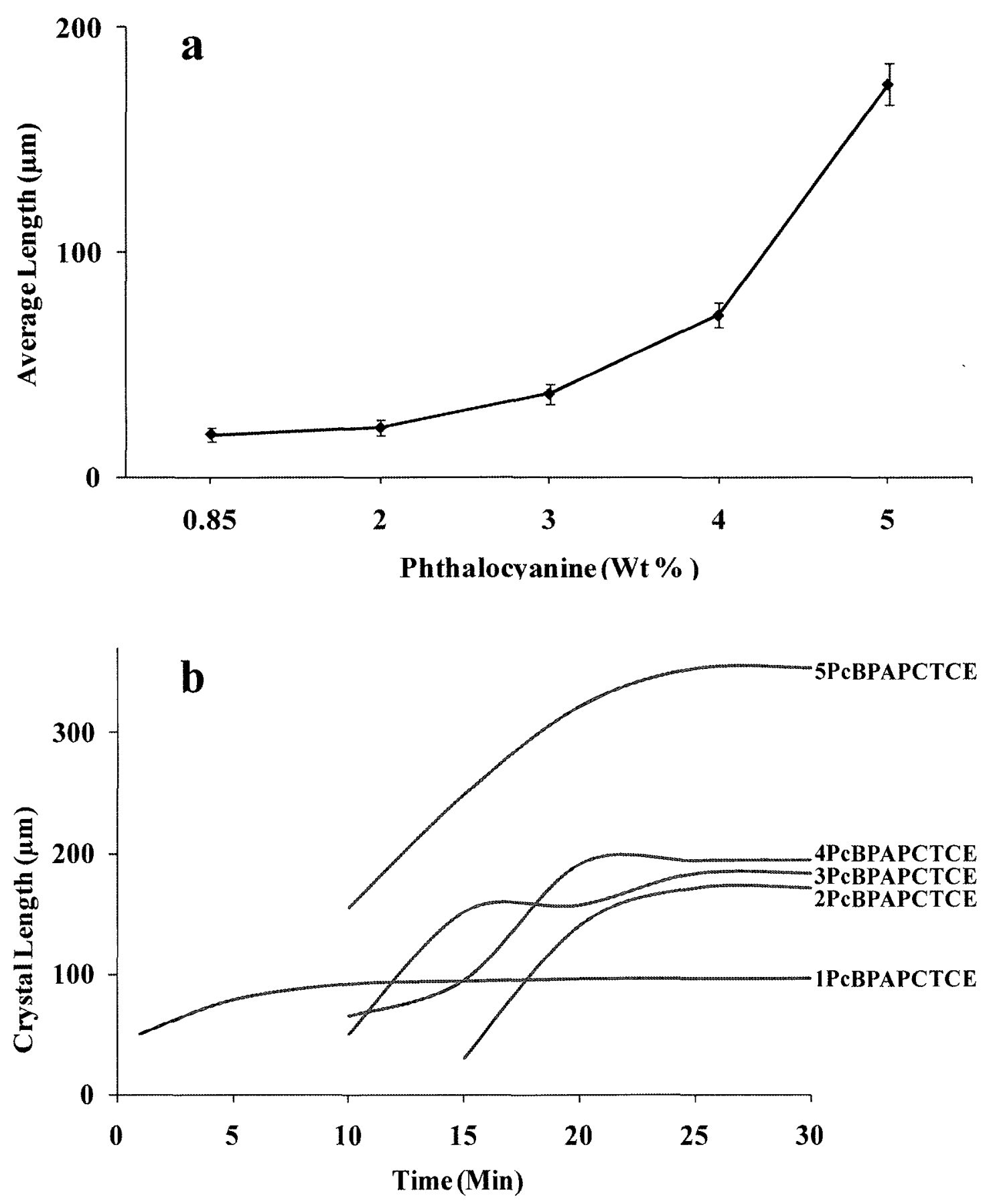

Figure 6.3: a) Average length of the crystals in films with different wt \% of Phthalocyanine b) Variation of the length of the crystals with time in situ in films made with tetrachloroethane with different concentrations of phthalocyanine in BPAPC. 


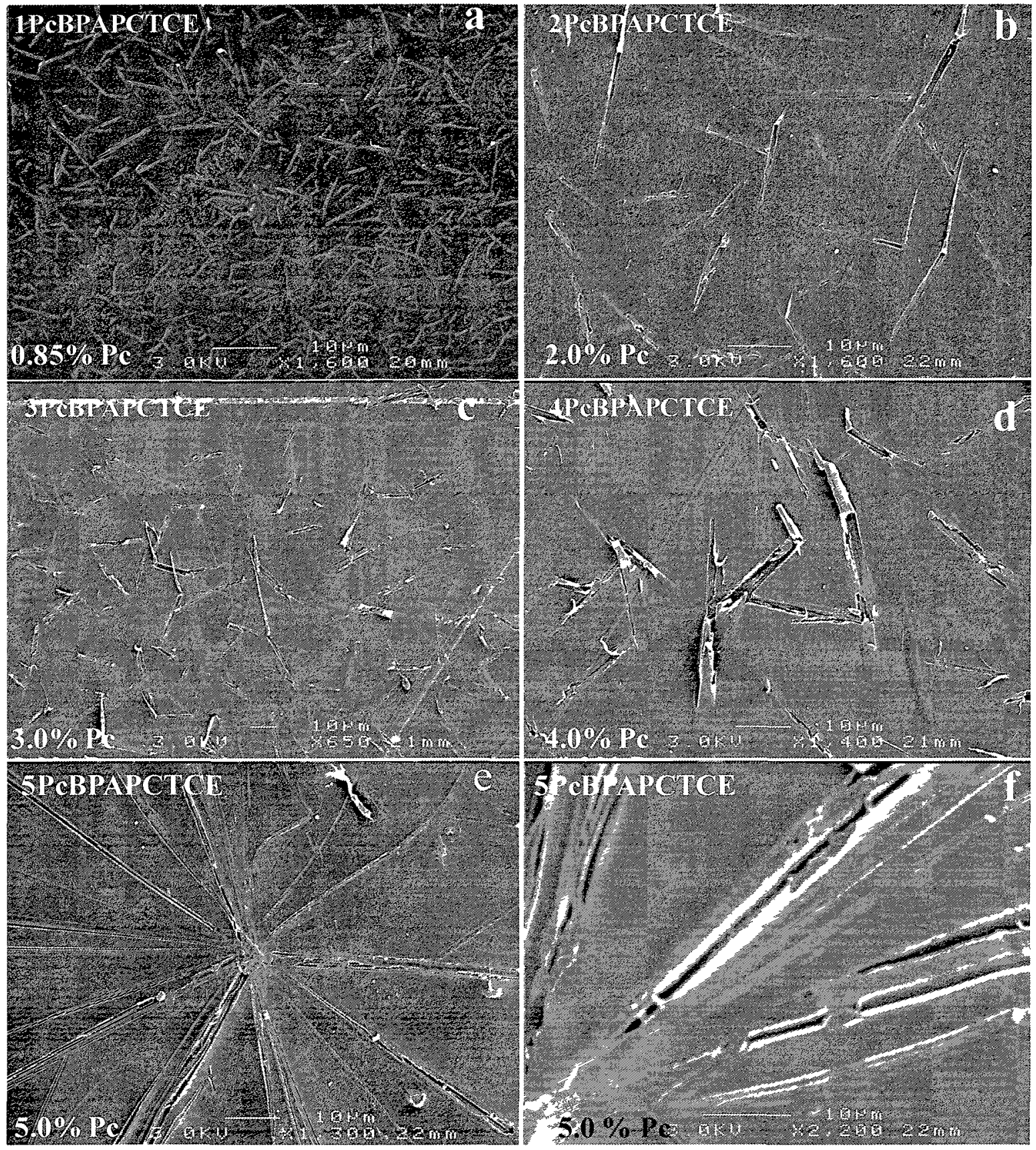

Figure 6.4: SEM of Pc/BPAPC/TCE films with (a-e): $0.85-5 \%$ concentrations of Pc. A higher magnification image of $5 \mathrm{Pc} / \mathrm{BPAPC} / \mathrm{TCE}$ is shown in $(\mathrm{f})$. 


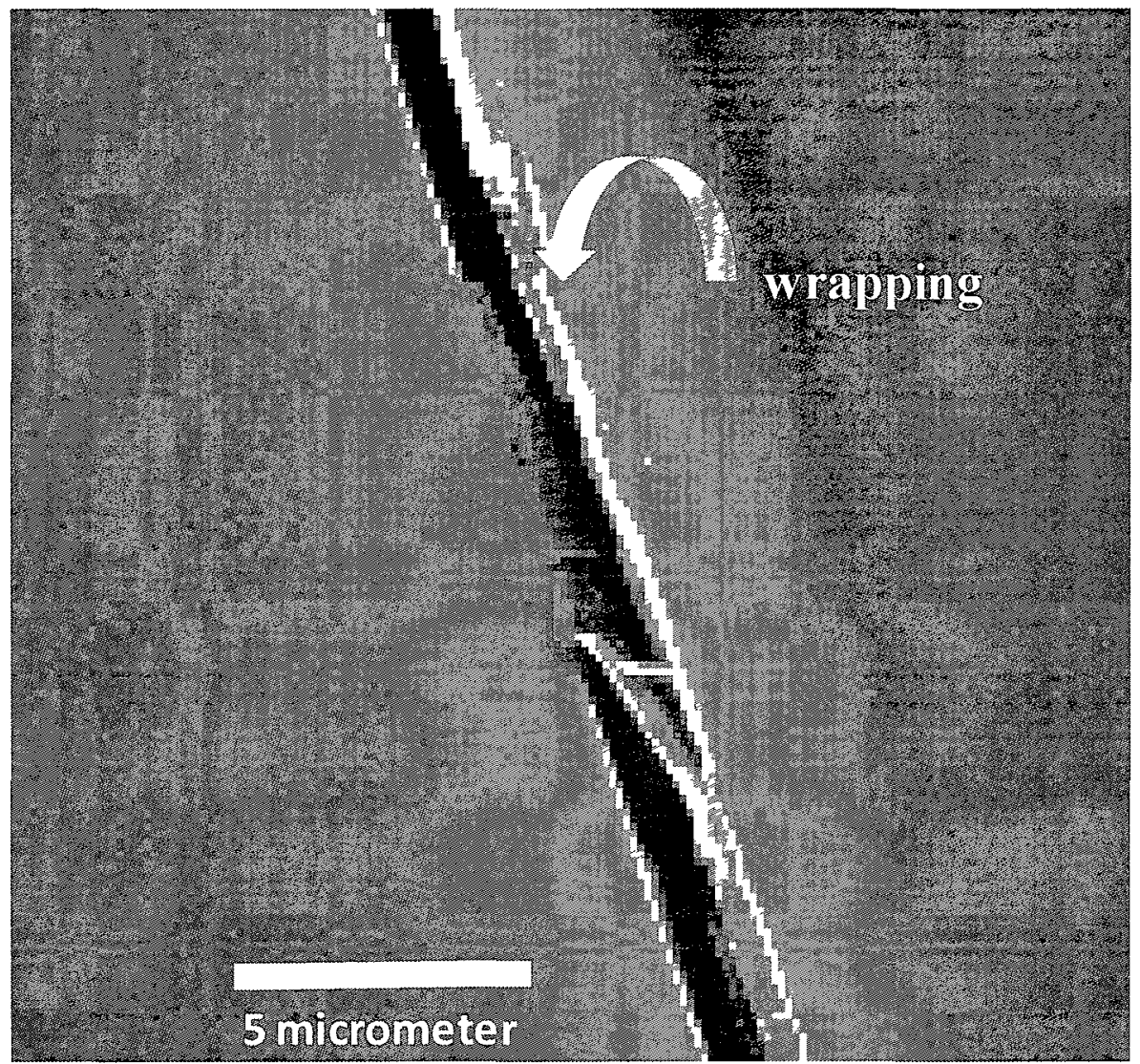

Figure 6.5: Tube formation of Phthalocyanine.

The SEM micrographs of Pc/BPAPC/TCE films are shown in figure 6.4. An interesting feature is clearly seen in figures $6.4 \mathrm{~d}-\mathrm{f}$. The crystals are tubular in shape. A SEM micrograph of $10 \mathrm{Pc} / \mathrm{BPAPC} / \mathrm{TCE}$ is shown in figure 6.5 , showing the wrapping during tube formation.

Without peripheral substitution. Pc is known to form needle-like crystals. Panina et $\mathrm{al}^{18}$ observed that both metal free and $\mathrm{Cu}-\mathrm{Pc}$, without peripheral substitution, forms needle-like crystals. Unlike the present work which is solvent-based, Panina et al grew the crystals by sublimation and these were not reported to be tubular. We are not aware of 
any report of tubular growth with unsubstituted phthalocyanine. With peripheral substitution, the Pc molecule becomes non-planar. Kojima et $\mathrm{al}^{30}$ and Drain et al ${ }^{31}$ discussed that the non-planarity of porphyrins with large peripheral substitution leads to saddle-like or dome-shape, and these serve as tectons for self-assembly into curved and tubular shapes (see scheme 1 of Kojima et al). The 1,4,8,11.15,18,22,25-Octabutoxy$29 \mathrm{H}, 31 \mathrm{H}$-phthalocyanine used here is non-planar, as seen from the crystal structures. ${ }^{25-27}$

A molecular model of this Pc is shown in figure 6.6. To verify if the tubular formation is characteristic of this substituted Pc, crystals were grown from a solution of Pc (by itself) in TCE. During the early stages of this study, stock solutions of Pc in TCE and chloroform were prepared to study the molar solubility. As the solvent slowly leaked out of the bottles over a period of weeks, crystals were seen on the wall, with different morphologies. Figure 6.7 shows that needle-like crystals had grown in the bottle in which TCE was used, and the SEM micrographs in this figure show the tubular formation of the crystals. The hollow nature is seen in figure $6.7 \mathrm{~b}$. The diameter of the tube is $\sim 30 \mu \mathrm{m}$ and the wall thickness is about $1-2 \mu \mathrm{m}$. Thus. the self-assembly in the form of tubular structure occurs even when the $\mathrm{Pc}$ is confined in a polymer matrix with this solvent. Since the saddle-like shape is considered to serve as a tecton for curved or tubular assembly, the question arises as to whether non-planarity of $\mathrm{Pc}$ is a requirement for tube formation. If this is a necessary and sufficient condition, such tube formation would be seen, irrespective of the solvent used for preparing the films. However, this is not the case as discussed below. 


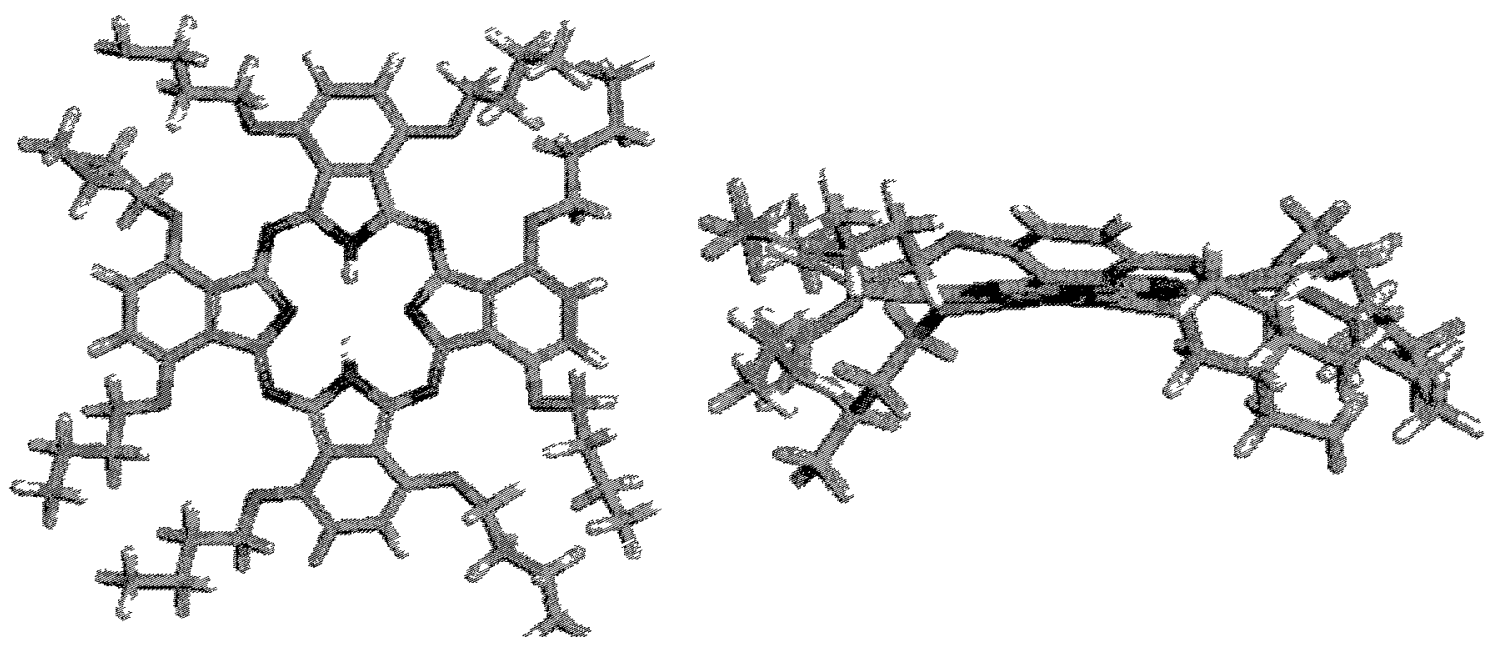

Figure 6.6: Projection of the phthalocyanine molecule after energy minimization (Accelrys Materials Studio ver 30 ) showing the non-planarity of the molecule with octabutoxy substituents. 


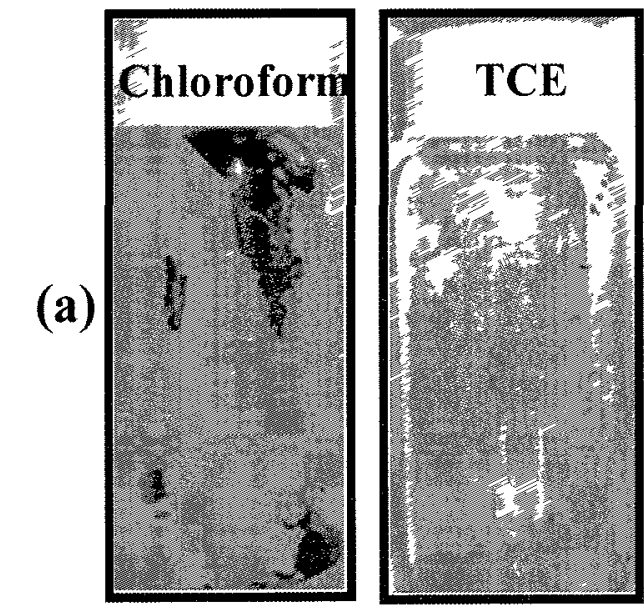

\section{Bottom of \\ TCE vial}

(b)
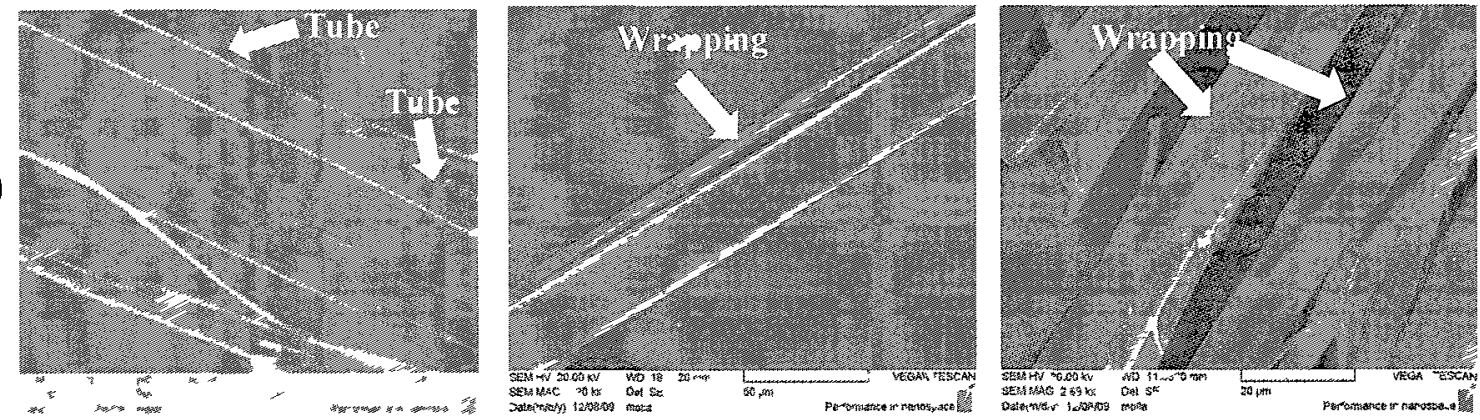

(c)
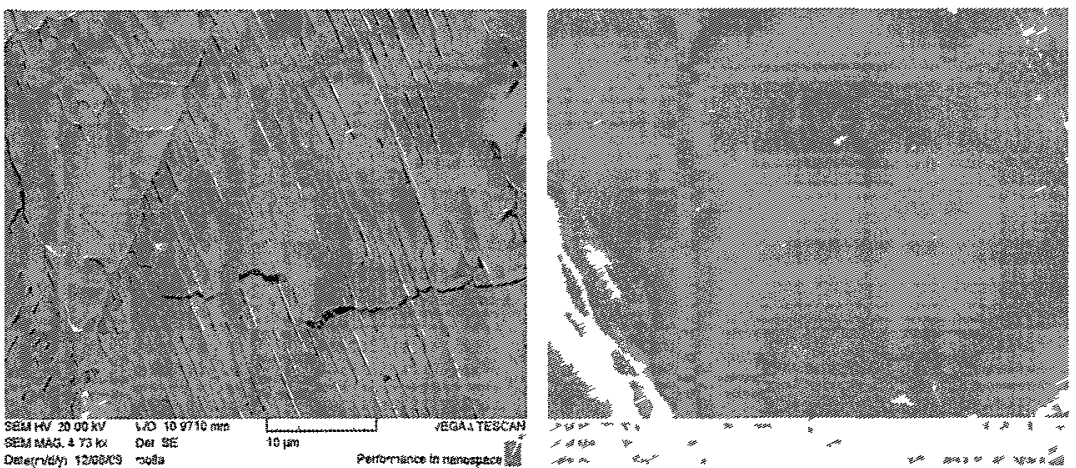

Figure 6.7 Crystals of phthalocyanine that slowly formed from chloroform and TCE solutions. (a) Saturated solutions were prepared to determine the molar solubility. Over time (weeks) crystals formed on the walls of the bottles, as above. SEM images of the crystals that formed inside the vial from TCE (b) and from chloroform (c) are shown. 


\section{Pc/BPAPC/CHL}

The Pc/BPAPC films prepared with chloroform show a very different morphology. The OM in figure 6.8 show that Pc crystallizes as droplets of a few microns in size, and no needle-like or tubular morphology is seen. With an increase in Pc concentration no significant change in morphology or shape was observed except that the number of droplets increases. Another feature is that these droplets can be seen in the transmission OM, but not with the SEM, leading to the conclusion that these are subsurface growth, and hence are not seen on the surface of the films. Figure $6.8 \mathrm{f}$ shows a confocal microscopy image of the 5Pc/BPAPC/CHL film. SEM micrographs (Figure 6.9) did not show any feature on the surface of the films. The same type of sub-surface assembly was seen in our previous studies ${ }^{16}$ on monocarbamate and biscarbamate in polycarbonate with chloroform as the solvent. These small molecules contain a single and double hydrogen bonding moieties, respectively, with alkyl side chains. Such crystallization within the bulk of the film was attributed to the fast self-assembly before the evaporation of the solvent and before the polymer film had dried. 

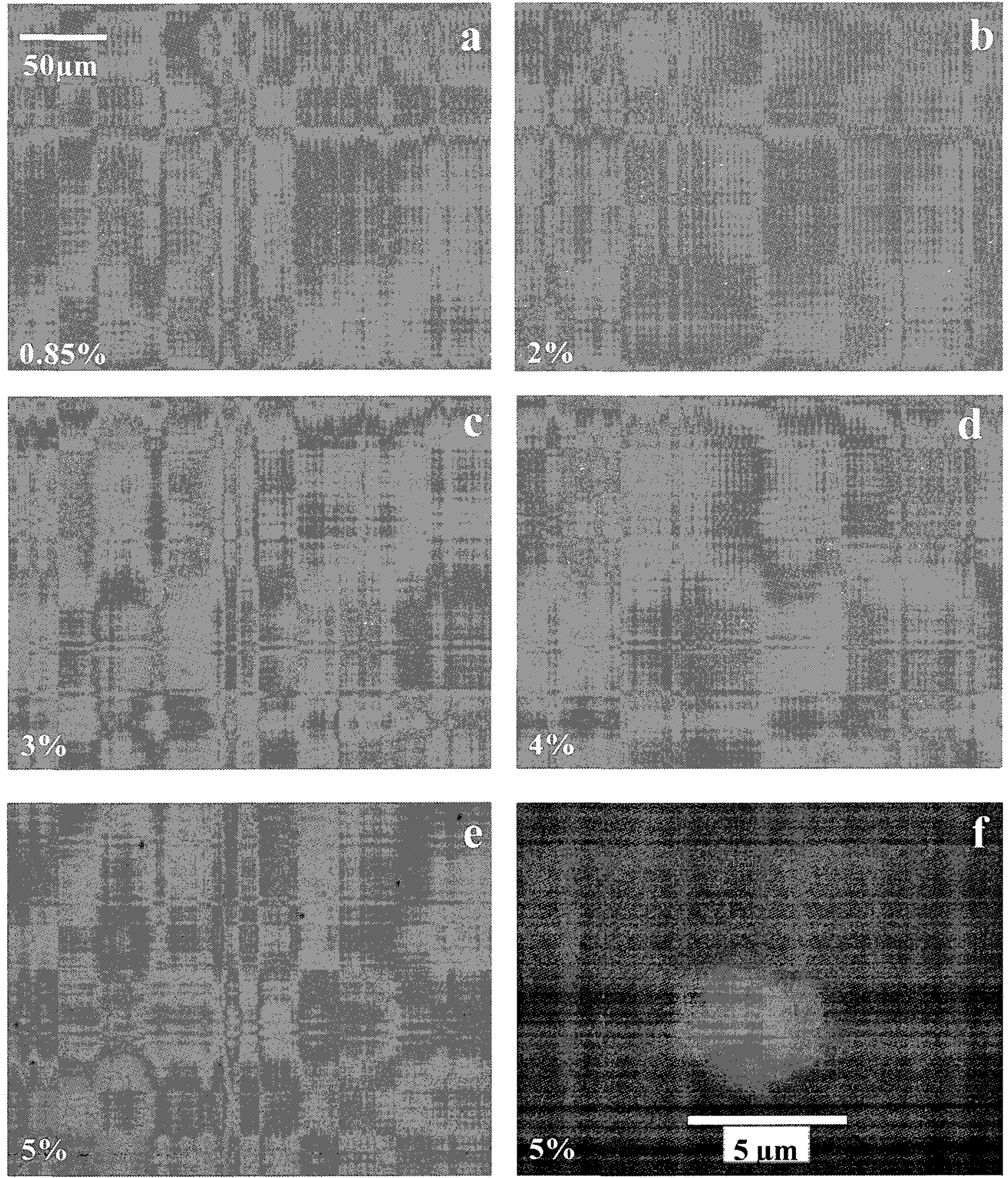

Figure 6.8: Optical micrographs of $\mathrm{Pc} / \mathrm{BPAPC} / \mathrm{Cl}$ films (a-e): $0.85-5 \%$. The scale bar is the same for a-e. Confocal microscopic image of $5 \mathrm{Pc} / \mathrm{BPAPC} / \mathrm{CHL}$ is also given (f). 

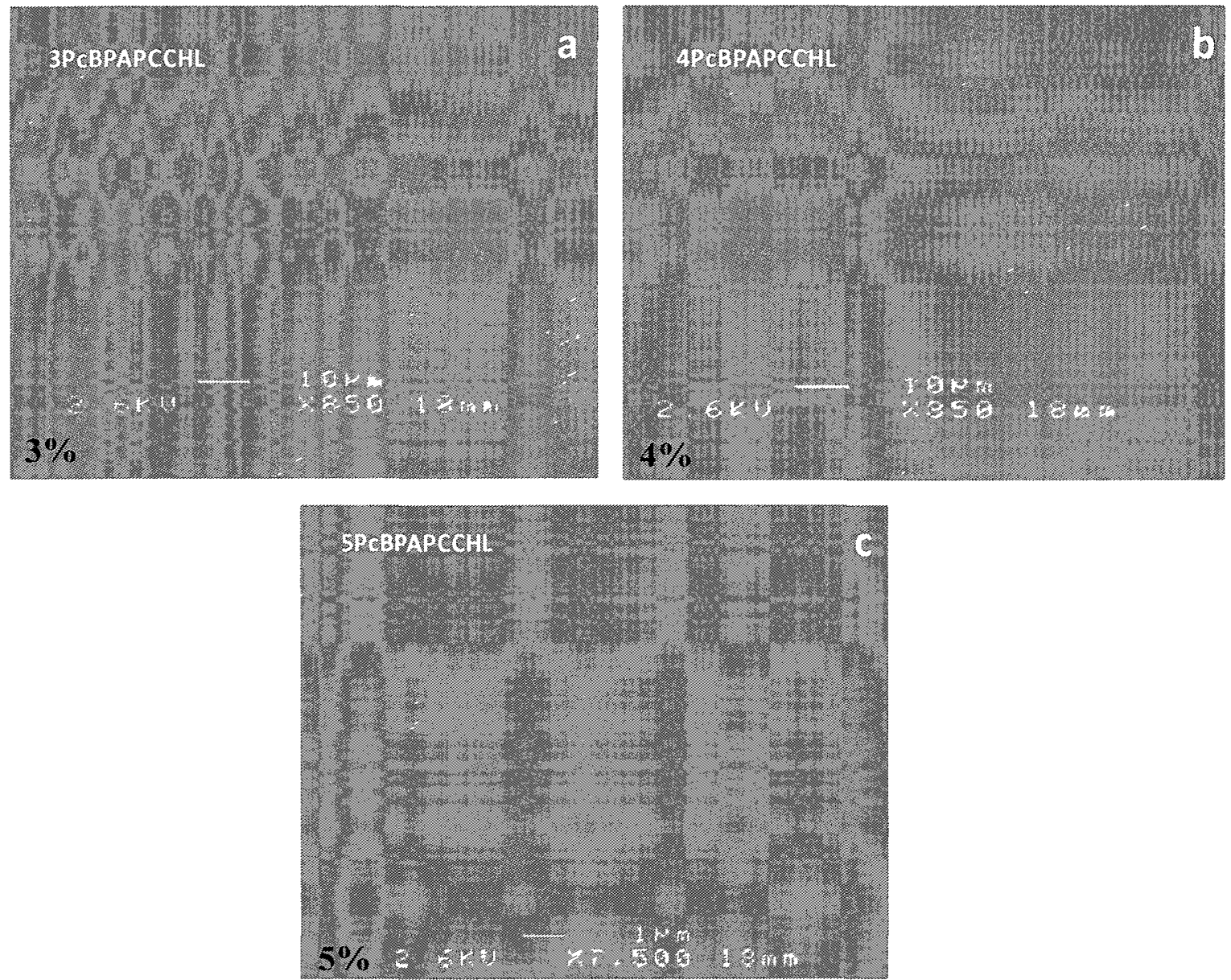

Figure 6.9: SEM of Pc/BPAPC/CHL films (a-c): 3-5\% (wt).

Our initial thought was that the conformation of the polymer in solution could be a directing agent for the morphology of the self-assembling molecule. It is known that the polycarbonate chain is extended (stretched out) in TCE and it adopts a globular conformation in chloroform. ${ }^{32}$ The extended conformation of the BPAPC chain in TCE could act as a template for the needle-like or tubular morphology of Pc, whereas the coiled conformation could result in droplets of Pc. However, this does not appear to be the case, as we found that the growth morphologies of Pc by itself, without the polymer, in these two solvents are different. 
As with TCE above, crystals of Pc (by itself) were grown in a chloroform solution. The SEM shown in Figure 6.7c does not exhibit any tubular or needle morphology, but stacks characteristic of columnar growth. Thus, the mode of selfassembly of Pc is different between TCE and chloroform as solvents and this is maintained in the polymer matrix as well.

\section{Pc/PMMA/TCE}

To study the effect of the polymer, if any, on the self-assembly of Pc, we used PMMA, a polymer with a chemical structure very different from that of BPAPC. The phase separated, self-assembled morphology of Pc in PMMA (with TCE as the solvent) was very similar to that in BPAPC. Figure 6.10 shows that even with $1 \%$ (wt) of Pc, needle-like crystals form throughout the film, similar to those seen in figure 6.2. The tubular morphology is clearly seen in Figures $6.10 \mathrm{f}$ and $\mathrm{g}$. The SEM of 5Pc/PMMA/TCE is shown in Figure 6.11. In this case again. tubular formation is seen. However, with chloroform as the casting solvent, droplets of Pc were seen in this case also, similar to Figure 6.8. These were again seen in the bulk of the film (with transmission OM) and not on the surface. 

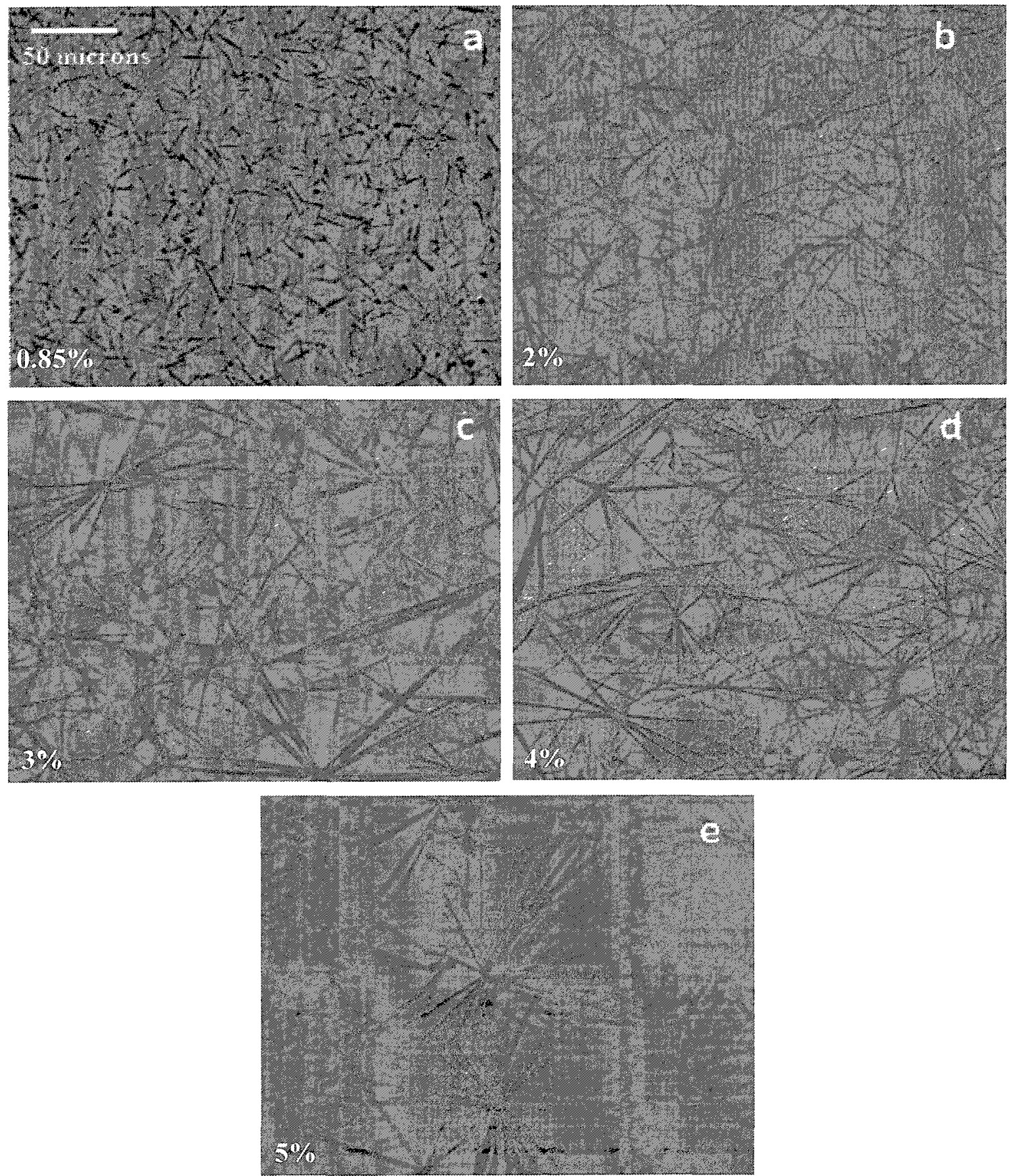

Figures continued in the next page 

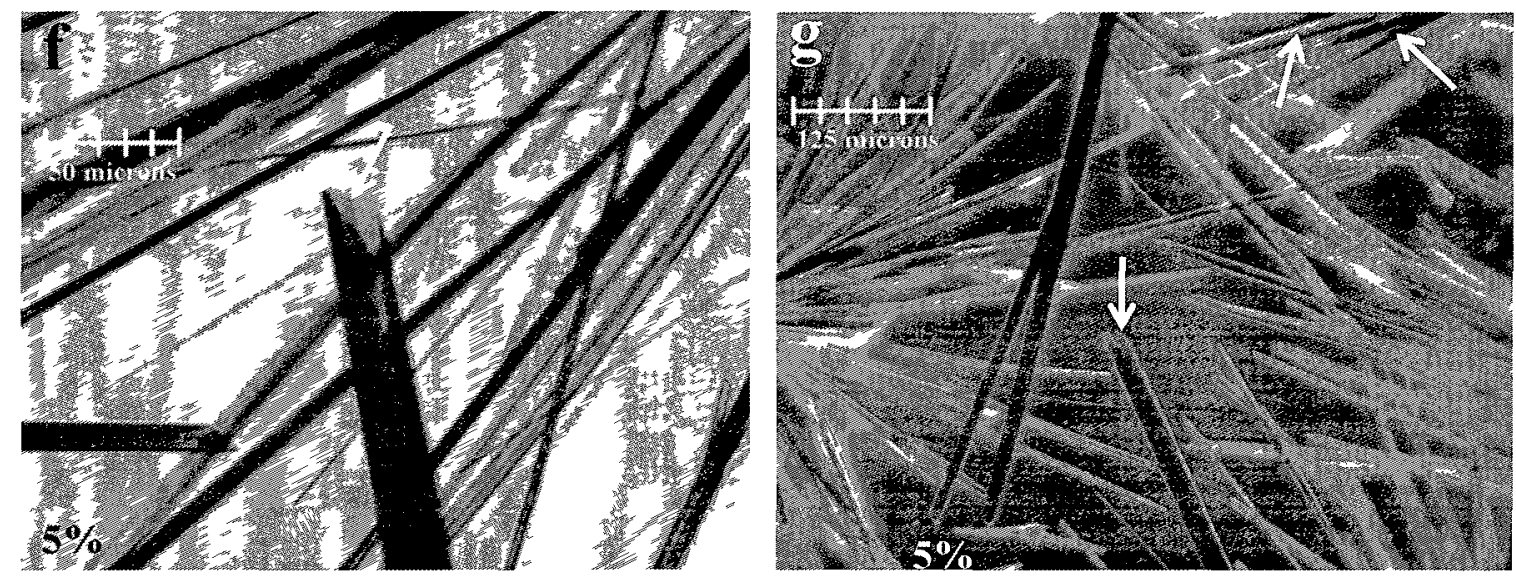

Figure 6.10: Optical micrographs of Pc/PMMA/TCE films with (a-e): 0.85 to $5 \%$ concentration of $\mathrm{Pc}$. The scale bar is the same for a-e. Figure $f$ and $g$ are bright and dark field $\mathrm{OM}$ images of $5 \mathrm{Pc} / \mathrm{PMMA} / \mathrm{TCE}$ films respectively to highlight the tube formation (marked with arrow)
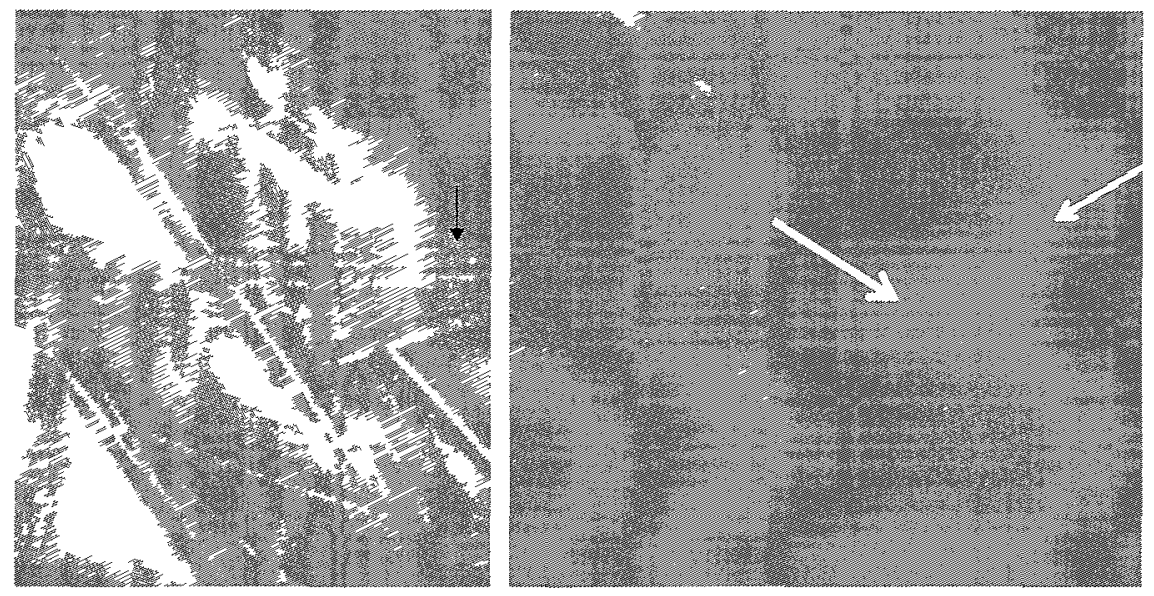

Figure 6.11: SEM of 5Pc/PMMA/TCE.

\subsubsection{Film Thickness}

The thickness of the films prepared with the electrical coater is about $14-18 \mu \mathrm{m}$.

To investigate if thinner films would show the same morphology of Pc self-assembly, we prepared films with a spin coater, at 500 and $1000 \mathrm{rpm}$. The corresponding films were 4- 
6 and 2-3 $\mu \mathrm{m}$ thick, respectively. Figure 6.12 shows the OM of 5Pc/BPAPC/TCE and 5Pc/BPAPC/CHL films prepared in this manner. It is seen that the same needle-like morphology is seen with TCE as the solvent, although the needles are shorter and thinner, as compared to those seen in figure 6.2. In this case again, the films prepared with chloroform show droplets of $\mathrm{Pc}$ in the bulk of the polymer matrix.
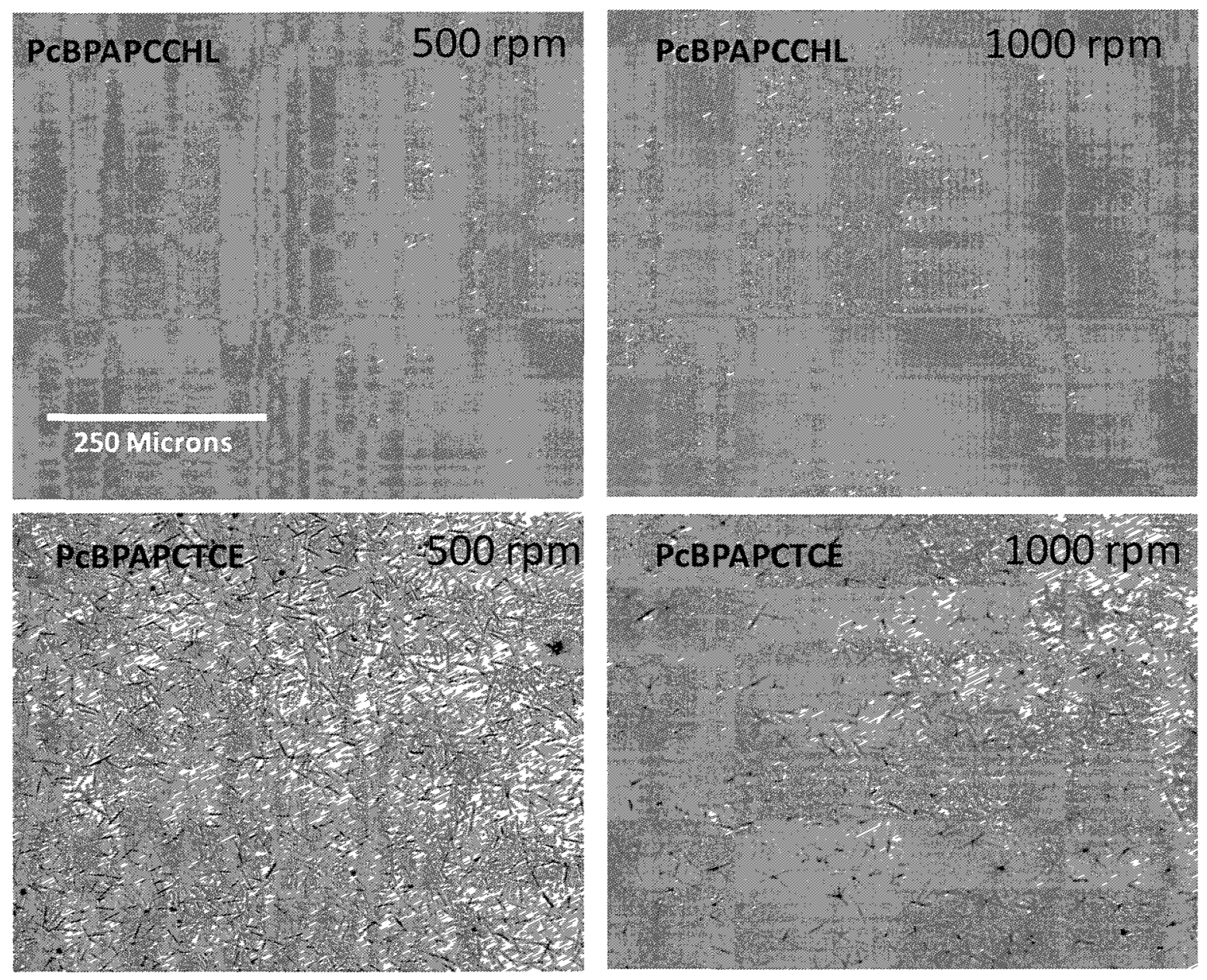

Figure 6.12: Optical micrographs of 5Pc/BPAPC/CHL (top) and 5Pc/BPAPC/TCE films prepared with spin coater at two different speeds. The scale bar is same for all. 


\subsubsection{NMR Spectra}

NMR spectra were recorded to investigate if the two solvents cause any conformational difference in Pc. If such a difference persists during crystallization, morphological differences could result. ${ }^{1} \mathrm{H}$ and ${ }^{13} \mathrm{C}$ NMR studies of $1,4,8,11.15,18$ hexadecyl-22,25-bis(6 hydroxyhexyl) phthalocyanine and its nickel derivative ${ }^{33}$ and $1,4,8,11,15,18,22,25$-octa-alkylphthalocyanines ${ }^{34}$ are known. Figures 6.13 and 6.14 show the ${ }^{1} \mathrm{H}$ and ${ }^{13} \mathrm{C}$ NMR solution spectra, respectively, of $\mathrm{Pc}$ in deuterated TCE and chloroform. The solutions for the study were made by dissolving $20 \mathrm{mg}$ of phthalocyanine in $600 \mu \mathrm{L}$ of respective solvents. It is seen that in all the regions of the spectra, the peaks are shifted slightly up-field with TCE as the solvent.
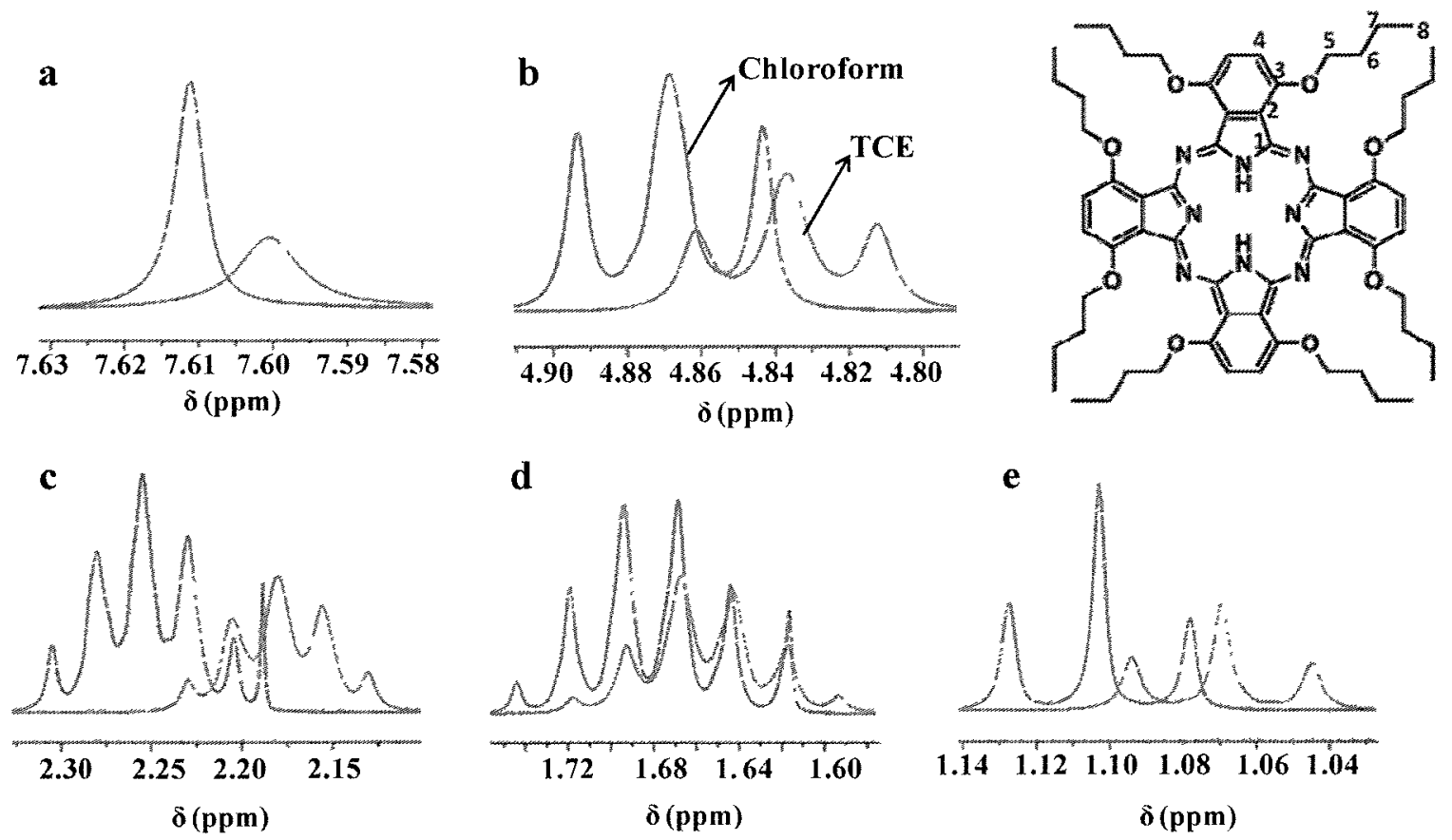

$$
\text { d }
$$
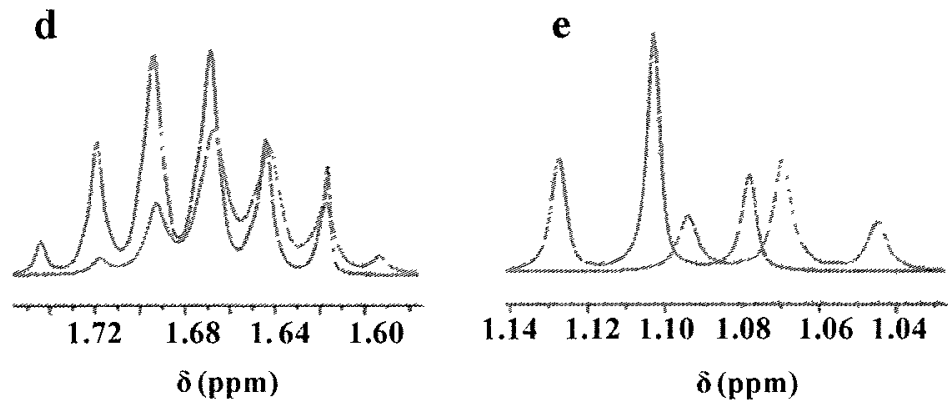

Figure 6.13: ${ }^{1} \mathrm{H}$ NMR of Phthalocyanine in TCE and Chloroform. 
$\mathbf{a}$

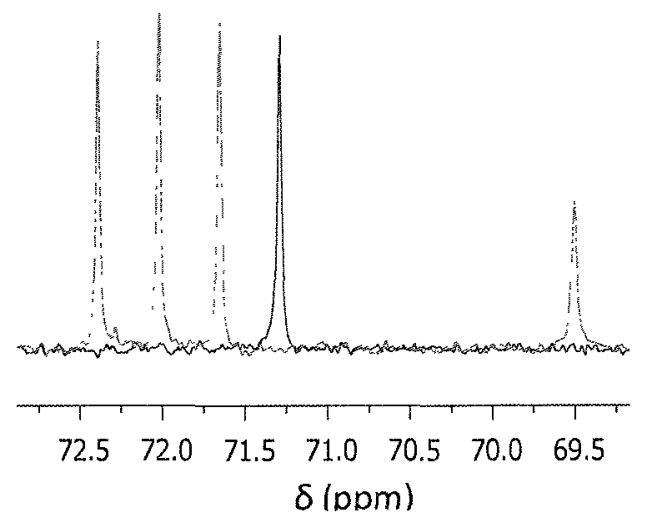

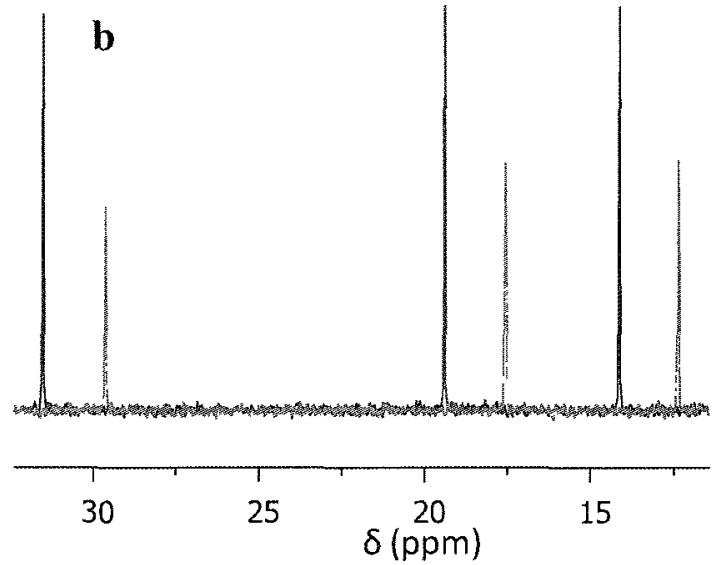

Figure 6.14: ${ }^{13} \mathrm{CNMR}$ of Phthalocyanine in TCE and Chloroform.

In the ${ }^{1} \mathrm{H}$ NMR a single peak is seen at 7.61 and $7.60 \mathrm{ppm}$ (figure 6.13-a) in chloroform and TCE respectively for all $\mathrm{C}-4$ hydrogens. As the aromatic hydrogens are far apart from the side chains we don't see any significant difference. The triplet corresponding to C-5 hydrogen (figure $6.13-\mathrm{b}$ ) is seen at 4.84 and $4.87 \mathrm{ppm}$ (de-shielded from normal alkyl substituted phthalocyanine due to the presence of alkoxy group) in TCE and chloroform, respectively. The peaks for C-6, C-7 and C-8 also show the same pattern, with the one for C-6 at 2.18 and $2.27 \mathrm{ppm}$ (figure 6.13-c), respectively for TCE and chloroform. Similar minor differences are seen in the ${ }^{13} \mathrm{C}$ NMR spectra as well (figure 6.14). Thus, NMR spectra do not show significant difference in the conformation of $\mathrm{Pc}$ in the two solvents.

\subsubsection{UV- Vis Spectra}

Figure $6.15 \mathrm{a}$ shows the UV-Vis solution spectra of Pc ( $0.001 \mathrm{~g}$ in $5 \mathrm{~mL}$ solvent) in TCE, with the Q-band with $\lambda_{\max }$ at 835 and $872 \mathrm{~nm}$. In previous studies, the narrow longer wavelength absorption has been attributed to a monomeric phthalocyanine 
compound, and the broad partially resolved absorption has been attributed to a dimer complex.

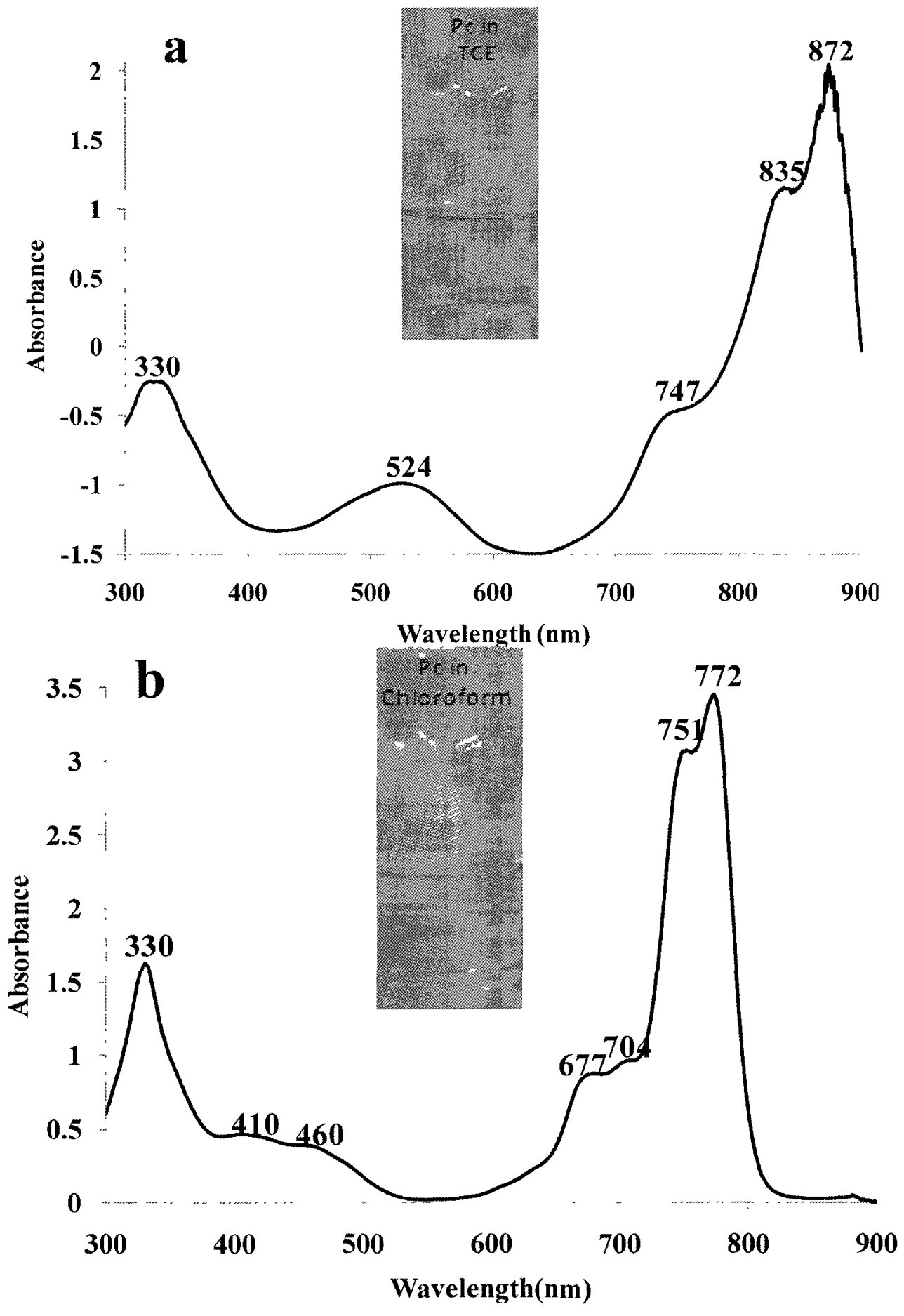

Figures continued in the next page 

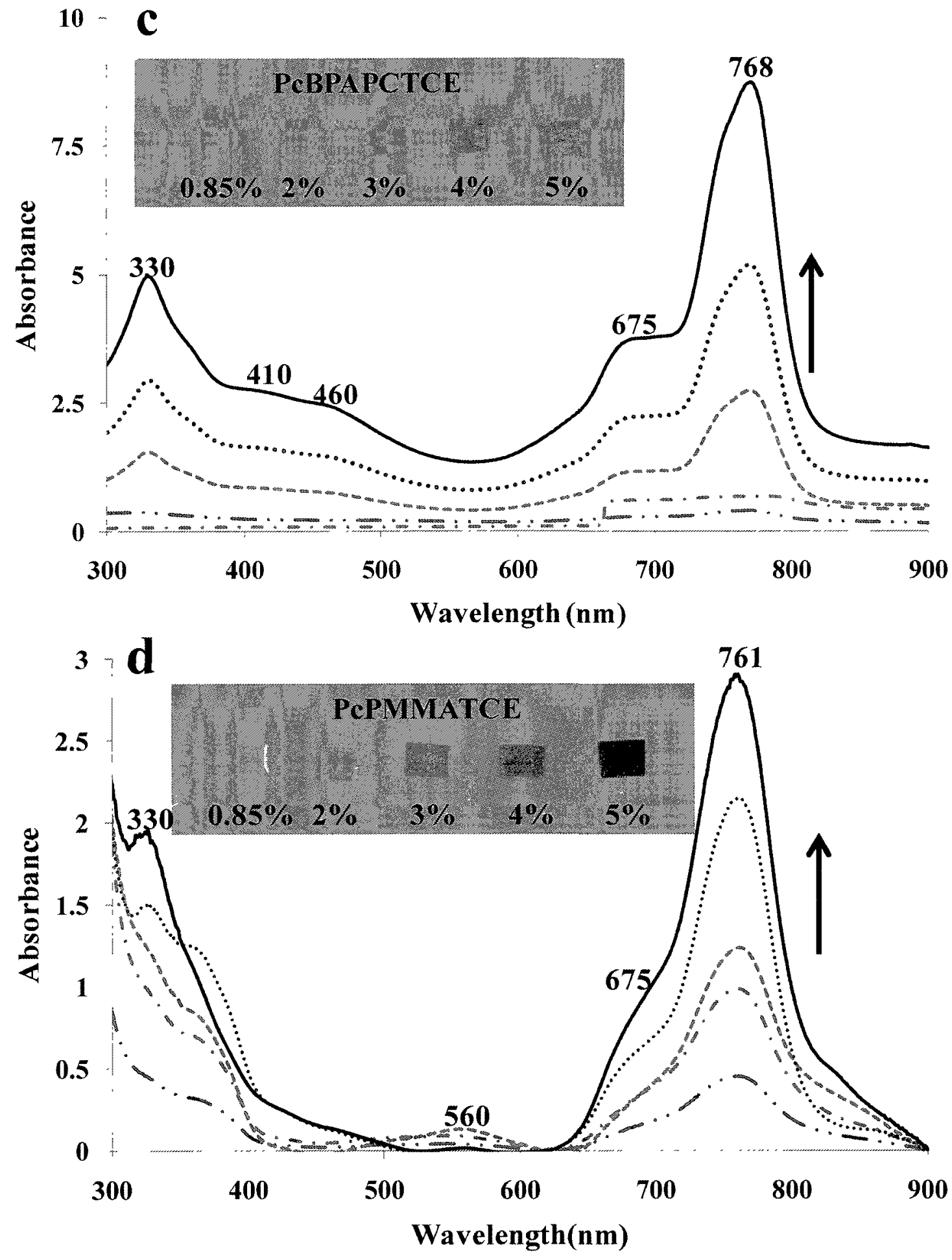

Figures continued in the next page 


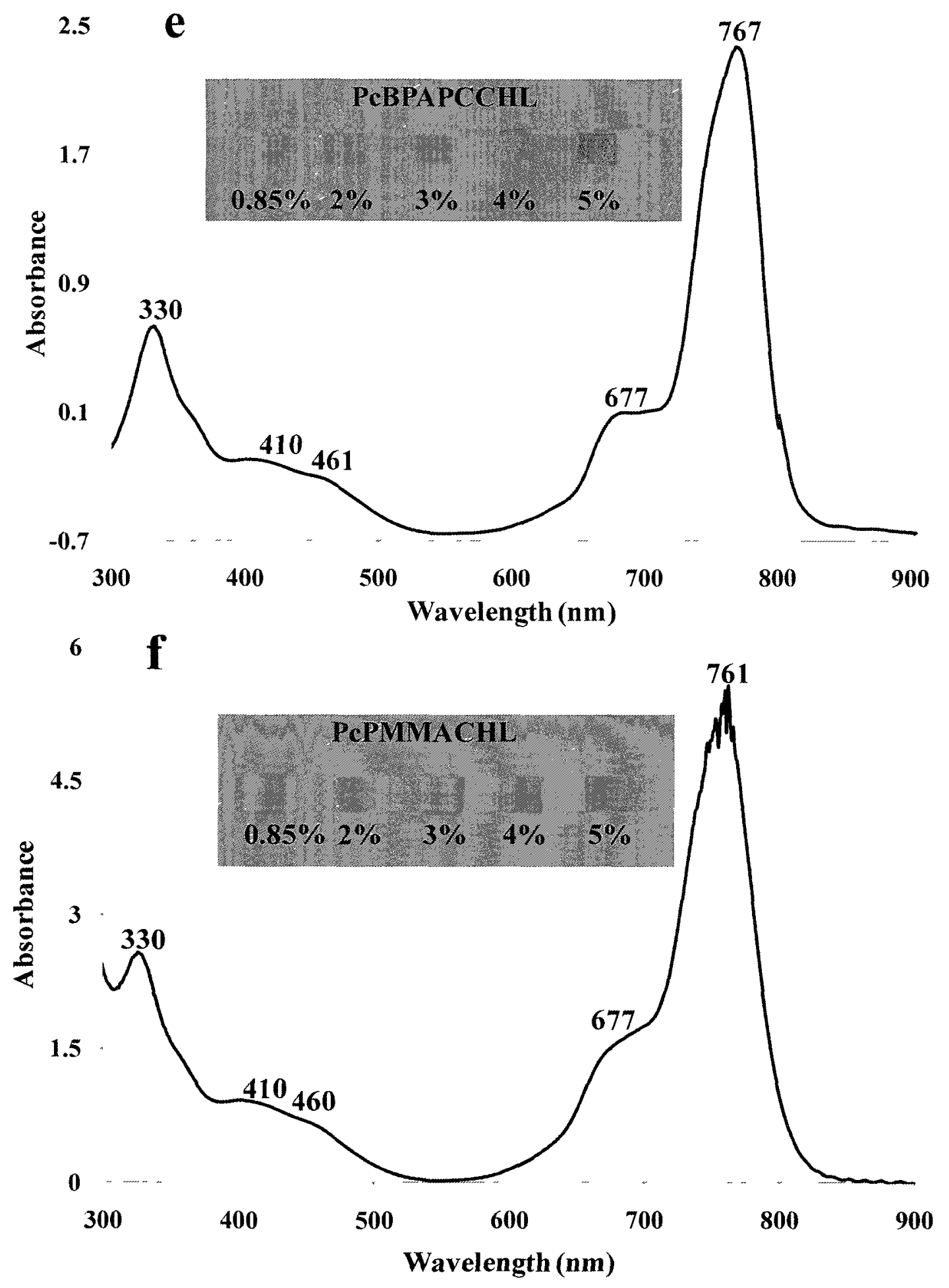

Figure 6.15: UV-Vis spectra of $\mathrm{Pc}$ in (a) TCE and (b) chloroform; (c) films of $\mathrm{Pc} / \mathrm{BPAPC} / \mathrm{TCE}$ and (d) Pc/PMMA/TCE with increasing concentration of Pc. (e) and (f) show the UV-Vis spectra of $\mathrm{Pc} / \mathrm{BPAPC} / \mathrm{CHL}$ and $\mathrm{Pc} / \mathrm{PMMA} / \mathrm{CHL}$ films respectively with $1 \%$ of Pc only. In this case, the intensity goes beyond the scale as the concentration increases. 
An intense Soret band is seen at $330 \mathrm{~nm}$. With the chloroform solution (figure $6.15 \mathrm{~b})$ the $\lambda_{\max }$ of these bands are at 772,751 and $330 \mathrm{~nm}$, respectively. The absorption at longer wave lengths in TCE compared to chloroform solutions is indicative of stronger $\pi$-assembly. The variation in the color of the solutions is shown in figures 6.16-6.18.

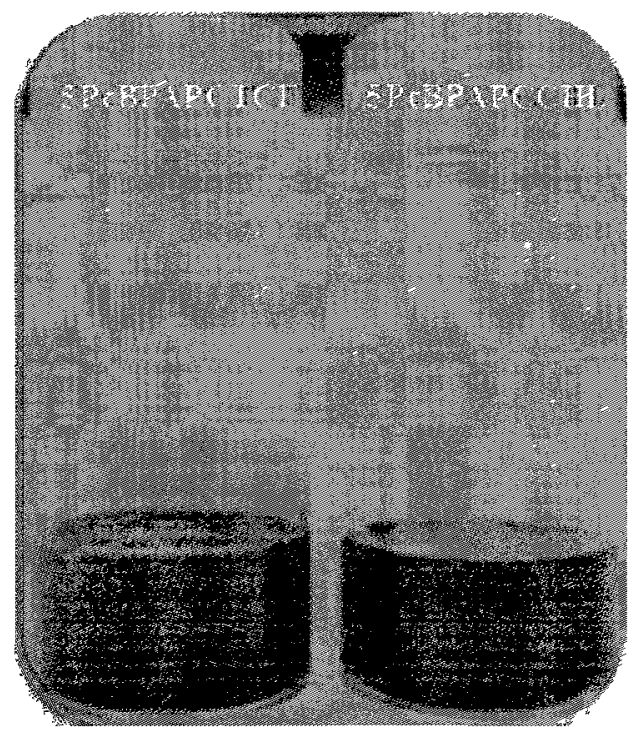

Figure 6.16: Photograph of PcBPAPC solution in TCE and Chloroform.

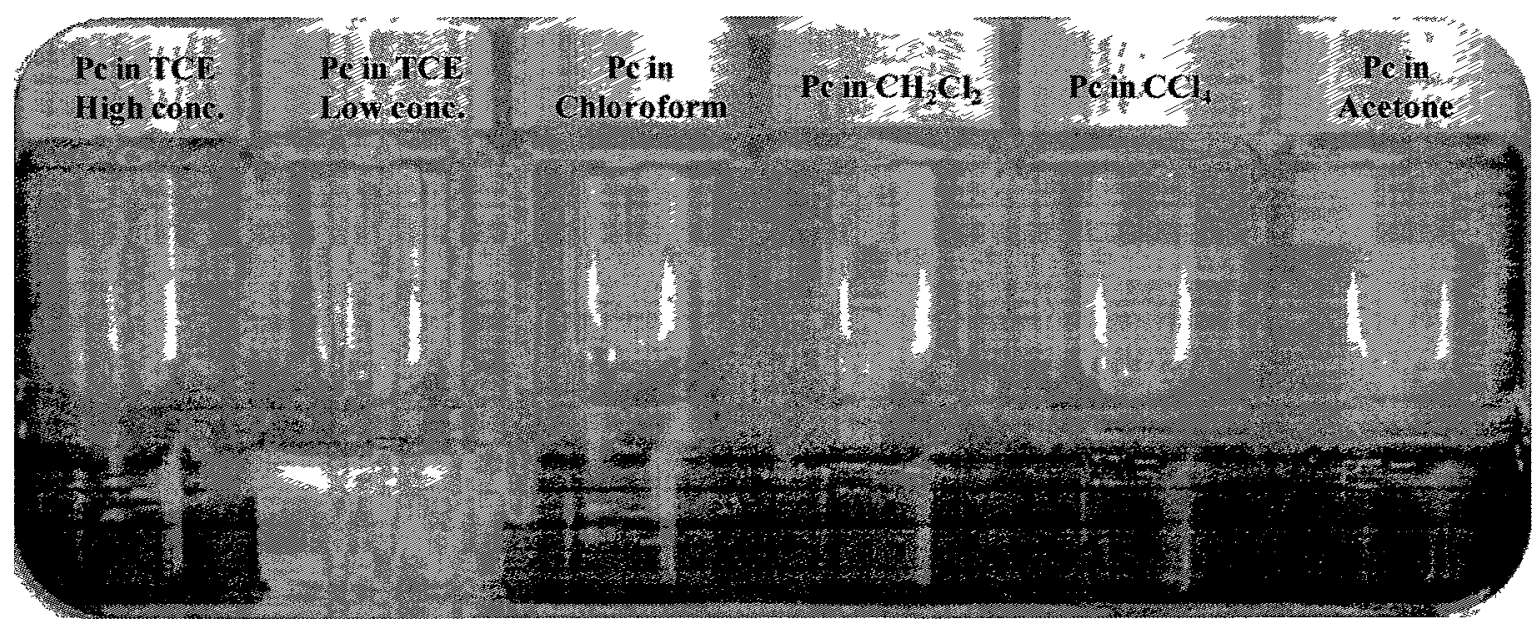

Figure 6.17: Photograph of Pc solution in different solvents after exciting at $366 \mathrm{~nm}$. Pc dissolved with high concentration doesn't show fluorescence. High concentration in TCE refers to $1 \mathrm{mg} / \mathrm{mL}$ and low concentration denotes $0.01 \mathrm{mg} / \mathrm{mL}$. 

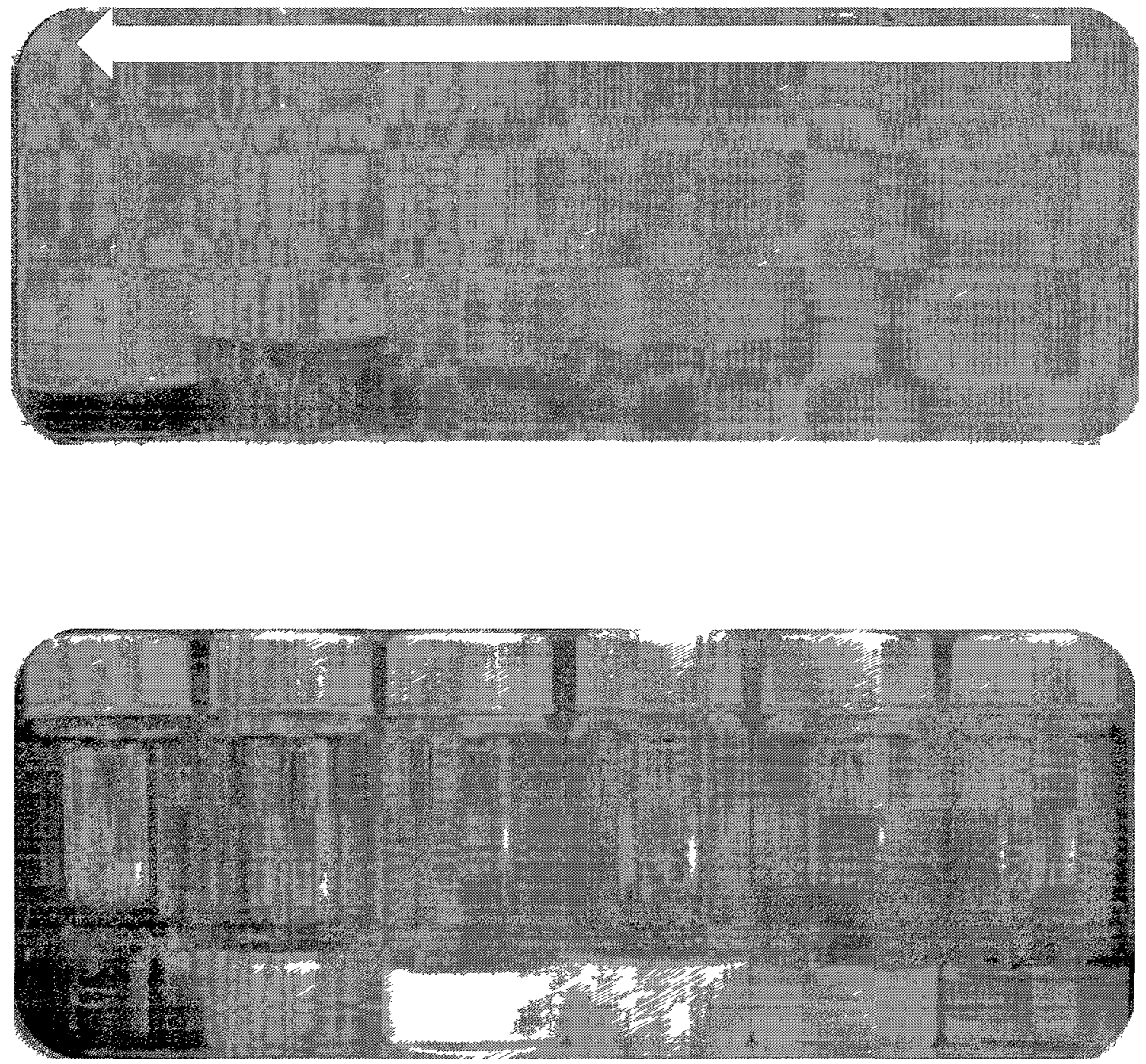

Figure 6.18: The color change of $\mathrm{Pc}$ dissolved in TCE (concentration increases in the direction of the arrow). The lower photograph was taken by exciting the solutions at $366 \mathrm{~nm}$. As we lower the concentration the solution in TCE starts fluorescing and with very low concentration fluorescent intensity goes down. For all the other solvents we don't see either any color change or fluorescence with concentration.

The color of the films made from these solvents varied with the concentration of $\mathrm{Pc}_{\mathrm{c}}$ in the polymer matrix (from black to green, as shown in the inset) and with the choice of the solvents (figure 6.15c-f). The UV-VIS spectra of the Pc/BPAPC/TCE films (figure $615 \mathrm{c})$ show absorption bands at 768,675 and $330 \mathrm{~nm}$. The blue shift of the Q-bands compared to the solution spectrum indicates $\mathrm{H}$-stacking of the Pc molecules. A gradual 
hyperchromic shift of the absorption is also seen with Pc concentration. Similar blue shift is also seen with Pc/PMMA/TCE films (figure $6.15 \mathrm{~d}$ ), with $\lambda_{\max }$ at $761 \mathrm{~nm}$ and a shoulder at $675 \mathrm{~nm}$. For the case of films made with chloroform, the spectra (figures $6.15 \mathrm{e}$ and $\mathrm{f}$ ) are shown only for $1 \%$ (wt) concentration of $\mathrm{Pc}$ in the polymer, as the absorption intensity goes out of scale for higher concentrations. But the $\lambda_{\max }$ for Pc in chloroform shows hypsochromic shift (blue shift) from $772 \mathrm{~nm}$ to $767 \mathrm{~nm}$ (Pc/BPAPC/CHL) and 761 nm (Pc/PMMA/CHL) in the films. This small shift compared with the shift caused in TCE solution may be related to the columnar structure formed from chloroform in a sealed vial. Thus, although NMR spectra do not show any difference in the conformation of $\mathrm{Pc}$ in the two solvents, the absorption spectra indicate that solvent plays a significant role for the $\pi$ - interaction driven self-assembly in solution and in the polymer matrix. We found that the morphologies with methylene chloride and carbon tetra chloride were similar to that with chloroform.

\subsubsection{X-ray Diffraction}

The results presented above show that the morphologies of $\mathrm{Pc}$ in films prepared with chloroform and TCE are different, irrespective of whether the polymer matrix is BPAPC or PMMA. NMR spectra did not show any significant difference in the conformation of PC in these two solvents. Although UV-Vis spectra show differences in the $\lambda_{\max }$ positions between the two solutions, the solid state (films) spectra are similar, indicating that the molecular packing does not differ. Thus, the molecular packing would not account for the difference in the morphology. We now discuss the influence of these solvents on some of the structural aspects of Pc itself, using powder X-ray diffraction. As 
mentioned above, X-ray crystal structure determination of the octa (butyloxy) substituted Pc has been reported by other authors. ${ }^{25-27}$ It is seen from figure 6.19 that the crystals of Pc from TCE are tubular, whereas those from chloroform are columnar stacks. 

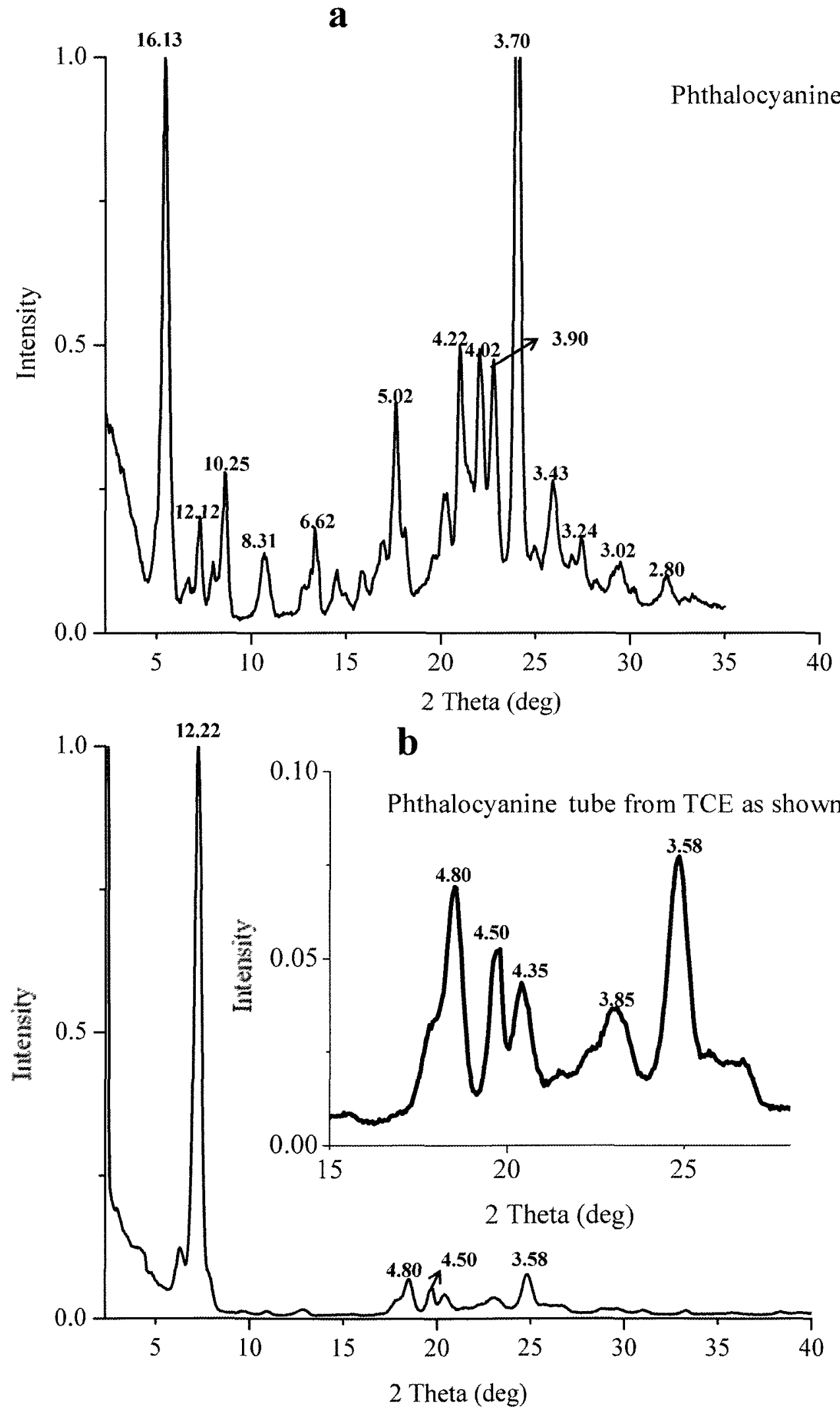


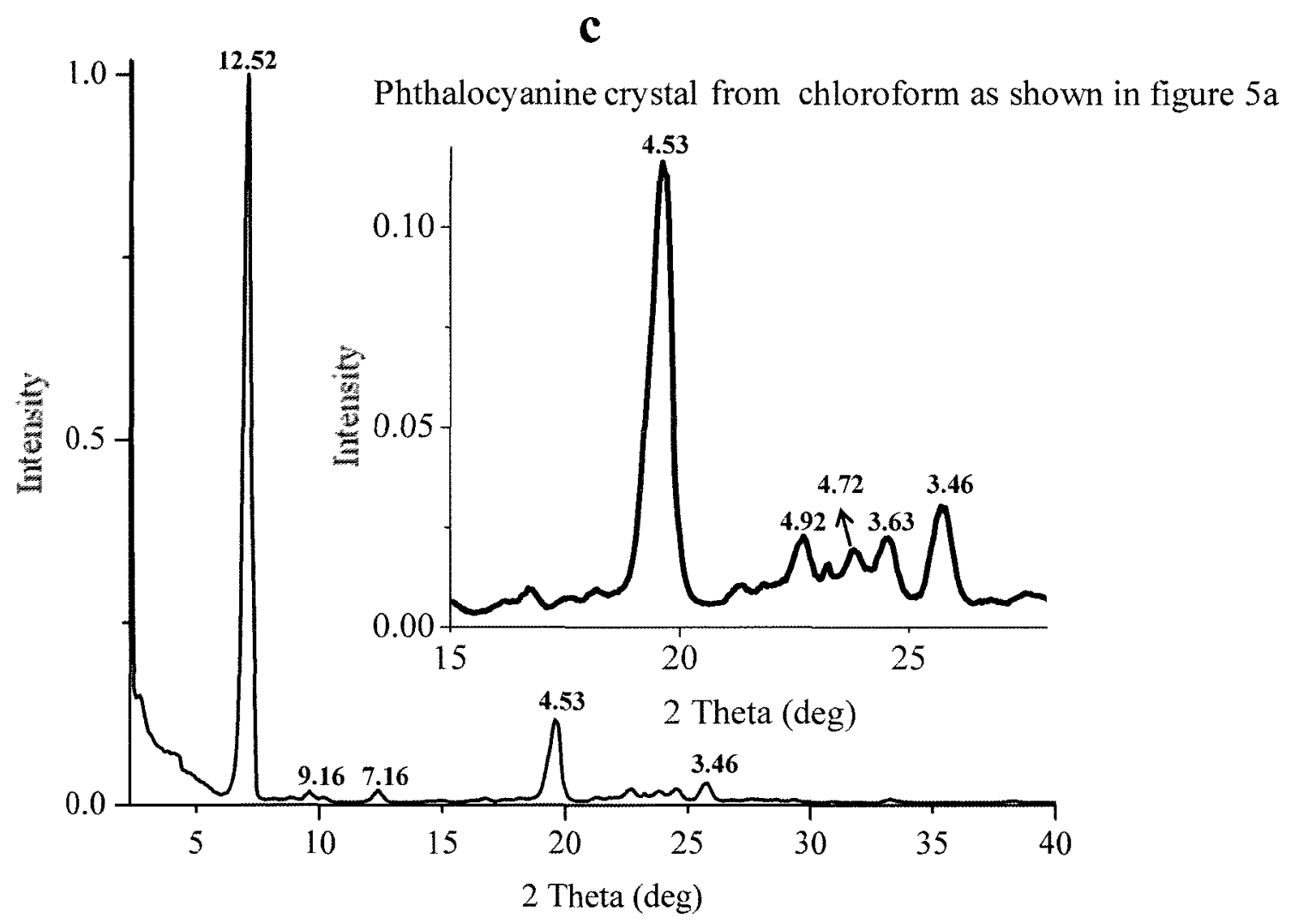

Figure 6.19: X-Ray powder diffraction profiles of (a) Pc as received (b) Pc crystallized from TCE and (c) from chloroform. (b) and (c) are from the bottles shown in Figure 6.5.

Figure 6.18 shows the powder X-ray diffraction traces of Pc as received (the prior history is unknown) and from the samples from the two bottles shown in figure 6.7. Each diffraction trace has been normalized with respect to the most intense reflection in the low angle region as $100 \%$ in intensity. The as-received sample (figure $6.19 \mathrm{a}$ ) shows $d$ spacings of $16.1,12.1,10.2,5.0,3.9$ and $3.7 \AA$, which are similar to those recorded by Gao et $\mathrm{al}^{27}$ for aggregates of Pc prepared by injecting a chloroform solution into methanol. Relating the crystal structure and the powder diffraction, the 16.1 and $12.1 \AA$ reflections were assigned to $(001)$ and (011) planes, respectively. ${ }^{27}$ With the samples retrieved from TCE and chloroform solutions (figure 6.7a), the diffraction pattern is 
significantly different from that recorded with the as-received sample. The noticeable feature is the presence of a single most intense peak with $d \approx 12.2-12.5 \AA$ in figure $6.19 \mathrm{~b}$ and $6.19 \mathrm{c}$, with the other reflections being of very low intensity. The single most intense reflection is indicative of one-dimensional organization. This reflection was assigned by Gao et $\mathrm{al}^{27}$ to the (011) planes of the crystal structure. Note that the $12.1 \AA$ reflection was of low intensity with the as-received sample (figure 6.19a). The crystal structures of substituted phthalocyanines ${ }^{25,26}$ show that there is only a weak interaction between the aromatic cores, and that the typical distance between them is about $8.5 \AA$. Note that the samples used for figures $6.19 \mathrm{~b}$ and $\mathrm{c}$ were not grown as single crystals from dilute solutions, but were simply taken from the bottles after the solvent evaporated.

The other weak reflections are shown in the inset in figures $6.19 \mathrm{~b}$ and $\mathrm{c}$. The reflections with $d=4.5,3.9$, and $3.5 \AA$ are seen in both, along with other weak reflections. The relative intensities of these differ, and this could be due to the non-random orientation of the samples in the X-ray beam.

Figure 6.20 shows the diffraction from the films of 5Pc/BPAPC/TCE, 5Pc/PMMA/TCE, 5Pc/BPAPC/CHL, and 5Pc/PMMA/CHL. With the films made from TCE, a single intense peak at $12.2 \AA$ is seen, similar to that in figure $6.19 \mathrm{~b}$, for both BPAPC and PMMA as the matrix polymer. However, with chloroform, a broad peak is seen with a spacing of 4.79 and $6.07 \AA$ for $5 \mathrm{Pc} / \mathrm{BPAPC} / \mathrm{CHL}$ and $5 \mathrm{Pc} / \mathrm{PMMA} / \mathrm{CHL}$ respectively. It is known ${ }^{27}$ that self assembled structures could give poor X-ray data. Due to this possibility, we refrain from concluding that the droplets (seen in the films prepared 
with chloroform) are amorphous. Note that the UV-Vis spectra of both types of films were similar.
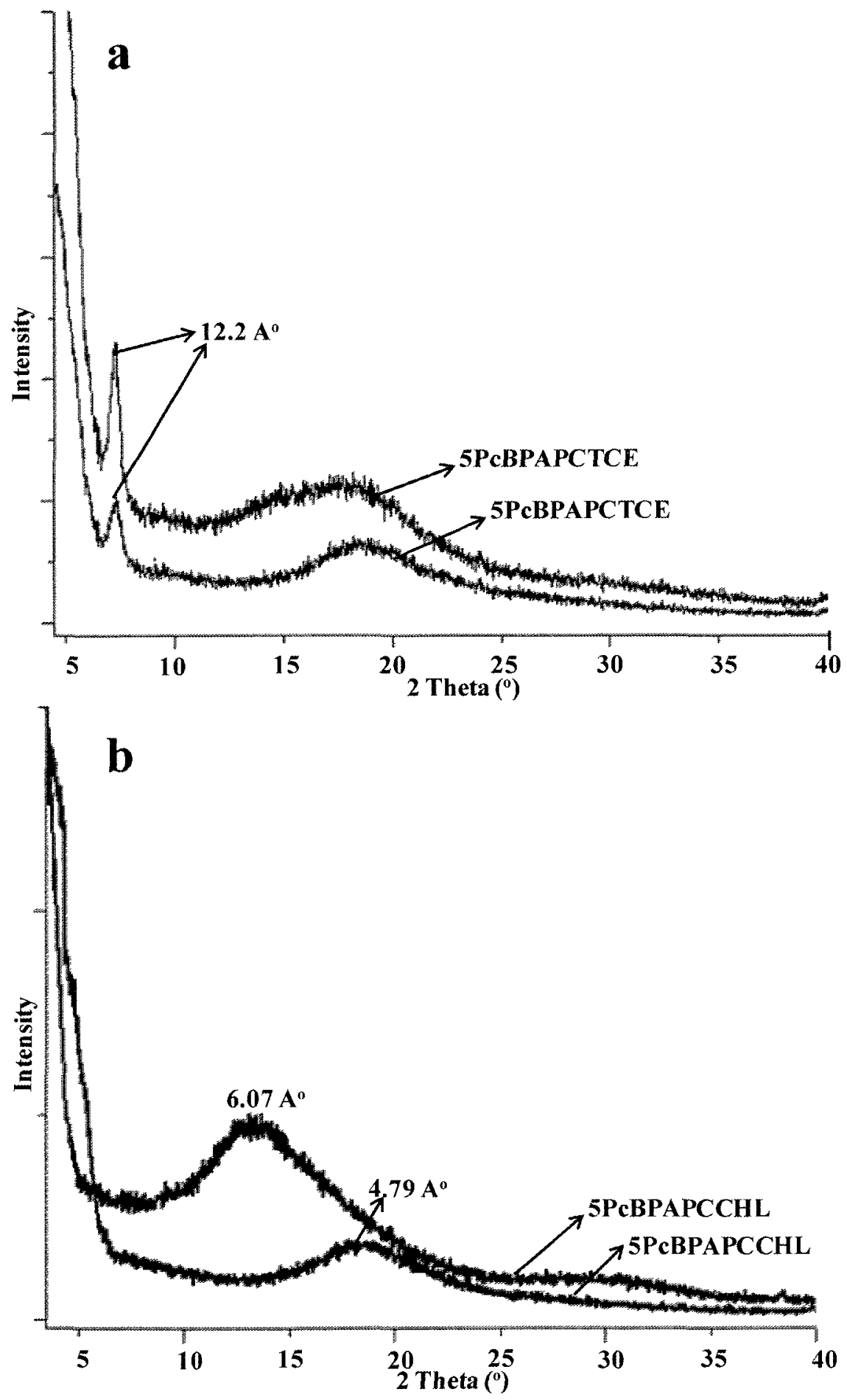

Figure 6.20: X-Ray powder diffraction profiles of (a) 5Pc/BPAPC/TCE and 5Pc/PMMA/TCE (a) and (b) 5Pc/BPAPC/CHL and 5Pc/PMMA/CHL films. 


\subsubsection{Viscosity of the Solutions and Growth Morphology}

The results of NMR, UV-Vis spectra and X-ray diffraction discussed above do not account for the tubular morphology in TCE-made films and the sub-surface droplet morphology observed with films made with chloroform. The same sub-surface morphology was seen in films made with methylene chloride $\left(\mathrm{CH}_{2} \mathrm{Cl}_{2}\right)$. The sub-surface morphology has also been observed in our previous studies on the self-assembly of carbamates in the polycarbonate matrix, when films were made with chloroform. ${ }^{[16]}$ In those cases, the self-assembly of the small molecules in polycarbonate was driven by hydrogen bonds. We attributed such sub-surface morphology to the rapid aggregation of the self-assembly molecules, even before their diffusion to the surface.

Since there is no structural (molecular packing) difference between the films made with TCE and chloroform, the difference in the morphology could be attributed to the difference in the diffusion of Pc in these two solvents. The viscosities of TCE and chloroform are 0.876 and $0.56 \mathrm{cp}$ respectively. For 5Pc/BPAPC/TCE and $5 \mathrm{Pc} / \mathrm{BPAPC} / \mathrm{CHL}$, these are 1.35 and $0.76 \mathrm{cp}$, respectively, with a solute concentration of $1 \%(\mathrm{wt})$. We hence believe that the higher viscosity of TCE solutions and the higher boiling temperature of TCE enable slow growth of the Pc molecules into tubular shape. Such tubular shape results when Pc crystallizes from TCE, even without the presence of the polymer (figure 6.7).

In a recent paper, Eddleston and Jones ${ }^{35}$ demonstrated the tubular growth of caffeine and a few other molecules, when the crystal growth is diffusion limited. When 
the crystal growth is rapid along one direction, and the diffusion of the molecules to the growing crystal face is limited, a central cavity forms within the crystal, leading to tubules. In the present case, the X-ray diffraction confirms the unidirectional growth of Pc by itself and in the polymer matrix, with the $12.2 \AA$ reflection being the most intense (Figures $6.19 \mathrm{~b}, 6.19 \mathrm{c}$, and $6.20 \mathrm{a}$ ). With the high viscosity of TCE solutions, the diffusion of Pc can be expected to be much slower than with chloroform. This leads to the tubular shape with TCE as the solvent. While we were able to measure the growth of the tubular needles with time (Figure 6.3b), the fast growth in chloroform could not be monitored with OM.

\subsection{Conclusions}

We have shown that the self-assembly of substituted Pc in polymer matrices leads to tubular morphology when the film is prepared with TCE and sub-surface droplet morphology with chloroform. Behaviour similar to that of chloroform was seen with methylene chloride also. The same morphology is seen with both BPAPC and PMMA as the matrix. In the absence of the polymer. Pc crystallized from TCE also shows tubular morphology, while it exhibits a columnar morphology with chloroform. The single most intense reflection (12.2 $\AA$ with TCE, $12.5 \AA$ with chloroform) seen in the X-ray diffraction of Pc crystallized from TCE as well as chloroform indicates the columnar stacking of the Pc molecules. However, the morphology is tubular when TCE is used. We attribute the difference to the higher viscosity of TCE, which enables slow growth leading to tubular morphology, while the instantaneous self-assembly in less viscous

chloroform leads to droplets. Similar to the case discussed by Eddleston and Jones ${ }^{35}$ the 
rapid growth along one direction, and the slow diffusion of the molecules to the growing crystal face (due to the high viscosity of TCE solutions) leads to tubular morphology, even in the presence of the polymer. While the saddle-like conformation of the substituted Pc acts as tecton for tubular shapes, and it is relevant at the nano-meter scale $^{30}$, the non-planarity does not seem to be a requirement for the micron-scale morphology. The effect of solvent discussed here provides a means to control the phaseseparated, self-assembled morphology. 


\subsection{References}

1. Philp, D.; Stoddart, J. F. Angew. Chem. Int. Ed. 1996, 35, 1154.

2. Lawrence. D. S.; Jiang, T.; Levett, M. Chem. Rev. 1995. 95, 2229.

3. (a) Lehn, J-M. Supramol. Chem., VCH, Germany, 1995. (b) Ikeda, M.; Nobori, T.; Schmutz, M.; Lehn, J-M. Chem. Eur. J. 2005, 11, 662.

4. Krische, M. J.; Lehn, J.-M. Struct. Bond. 2000, 96, 3.

5. (a) Beijer, F. H.; Kooijman, H.; Spek, A. L.; Sijbesma, R. P.; Meijer, E. W. Angew. Chem. Int. Ed. 1998, 37, 75. (b) Lehn, J-M. Makromol. Chem. Macromol. Symp. 1993, $69,1$.

6. (a) Murase, T.; Horiuchi, S.; Fujita, M. J. Am. Chem. Soc. 2010, 132. 2866. (b) Yoshizawa, M.; Klosterman, J. K.: Fujita,M. Angew. Chem. Int. Ed. 2009, 48, 3418. (c) Kawamichi, T.; Kodama, T.; Kawano, M.; Fujita, M. Angew. Chem. Int. Ed. 2008, 47, 8030. (d) Yoshizawa, M.; Fujita, M. Pure Appl. Chem. 2005, 77, 1107. (e) Yoshizawa, M.; Takeyama, Y.; Okano, T.; Fujita, M. J. Am. Chem. Soc. 2003, 125, 3243.

7. (a) Zhu, J.; Wang, M. J. Macromol. Sci. B, 2008, 47, 401. (b) Chen, Yu-F.; Woo, E.M.; Li, Shu-H. Langmuir, 2008, 24, 11880.

8. (a) Miura, T.; Mikami, M. Phys. Rev. E. 2007, 75, 031804. (b) Mataz, A.; Gregory B., M.; J. Phys. Condens Mat. 2005, 17, 461.

9. (a) Shin. K.; Obukhov, S.; Chen, J.-T.; Huh, J.; Hwang,Y.; Mok, S.; Dobriyal, P.; Thiyagarajan, P.; Russell, T. P. Nature Materials 2007, 6, 961. (b) Clifford L., H.; Lovejeet, S.; Peter J.,L.; Polym. Mater. Sci. Eng. 2004, 90, 350.

10. (a) Echeverria, C.; Kapral, R.; J. Chem. Phys. 2010, 132, 104902. (b) Kalogeras, I. M.; Neagu. E.R. Eur. Phys. J. Part E. 2004. 14, 193. 
11. (a) Yorulmaz, M.; Kiraz, A.; Demirel, A. L. J. Phys. Chem. B. 2009, 113, 9640. (b) Ye, H.-M.; Peng , M.; Xu, J.; Guo , B.-H.; Chen , Q.; Yun , T.-L.; Ma, H. Polymer, 2007, 48, 7364. (c) Lu, J.; Mirau, P.A.; Tonelli, A.E. Prog. Polym. Sci. 2002, 27, 357. (d) Rallabandi, P.S.; Ford, D. M. J. Membrane Sci. 2000, 171, 239. (e) Alan, E.T. Makromol. Chem. Macromol. Symp. 1993, 65, 133.

12. Gensel, J.; Liedel, C.; Schoberth, H.G.; Tsarkova, L. Soft Matter 2009. 5. 2534.

13. Tuteja, B.; Khan, F.; Sundararajan. P. Macromol. Chem. Phys. 2005, $206,354$.

14. Dudowick, J.; Douglas, J.F.; Freed, K. F.; J. Phys. Chem. B. 2009, 113, 3920.

15. (a) Khan, F.; Khanna, S.; Hor , A-M.; Sundararajan, P. R. Can. J. Chem. 2010, 88, 247. (b) Khan, F.; Hor, A.-M.; Sundararajan, P. R. Synthetic Met. 2005, 150, 199. (c) Khan, F.; Hor, A.-M.; Sundararajan, P. R. Pure Appl. Chem. 2004, 76, 1467. (d) Khan, F.; Hor, A.-M.; Sundararajan, P. R. J. Phys. Chem. B 2004, 108, 117.

16. (a) Islam, M. R.; Sundararajan, P. R. Can. J. Chem. 2008, 86, 600. (b) Tuteja, B.; Moniruzzaman, M.; Sundararajan, P. R. Langmuir 2007, 23, 4709.

17. (a) Yao, D.; Tuteja, B.; Sundararajan, P. R. Macromolecules 2006, 39, 7786. (b) Yao, D.; Bender, T.; Gerroir P. J.; Sundararajan, P. R. Macromolecules 2005, 38, 6972.

18. Panina, N.; van de Ven, R.; Janssen, F.F.B.J.; Meekes, H.; Vlieg,E.; Deroover, G. Cryst. Growth Design 2009, 9, 840.

19. Engel, M.K. in The Porphyrin Handbook, Kadish, K. M.; Smith, K.M.; Guilard, R. Eds. 2003, volume 20, Chapter 122.

20. Chambrier, I.; Cook, M.J.; Helliwell, M.; Powell, A. K. J. Chem. Soc., Chem. Commun. 1992, 5, 444. 
21. Cook, M.J.; McMurdo, J.; Powell A. K. J. Chem. Soc., Chem. Commun. 1993,11, 903.

22. Hammond, R. B.; Roberts, K.J.; Docherty, R.; Edmondson, M.; Gairns, R. J. Chem. Soc., Perkin Trans. 2, 1996, 8, 1527.

23. Chambrier, I.; Cook. M.J.; Wood, P.T. Chem. Commun. 2000, 21, 2133.

24. Bergesen, K.; Haugland, L.; Cook, M. J.; Bryant, G.C. J. Porphyrins Phihalocyanines. 2000, 4, 665.

25. Cai, J.; Wang, J.; Huang, J.; Chen, N. Chinese Sci. Bull. 2002, 77, 644.

26. Wang, J.; Huang, J.; Cai, J.; Chen, N. Chinese. J. Struct. Chem. 2002, 21, 617.

27. Gao, Y.; Chen, Y.; Li, R.; Bian, Y.; Li, X.; Jiang, J. Chem. Eur. J. 2009, 15, 13241.

28. (a) Duzhko, V.; Singer, K. D. J. Phys. Chem. C 2007, 111, 27. (b) Prince, B.J.; Williamson, B.E.; Reeves, R.J. J. Lumin. 2001, 93, 293. (c) Ribeiro, A.O.; Biazzotto, J.C.; Serra, O.A. J. Non-Cryst. Solids 2000, 273, 198. (d) Cook, M. J. J. Mater. Chem. 1996, 6, 677 .

29. (a) Diaz, D. D.; Cid, J. J.; Vazquez, P.; Torres, T. Chem. Eur. J. 2008, 14, 9261. (b) Díaz, D. D.; Torres, T.; Zentel. R.; Davis, R.; Brehmer, M. Chem. Commun. 2007, 22, 2369.

30. Kojima, T.; Harada, R.; Nakanishi, T.; Kaneko, K.; Fukuzumi, S. Chem. Mater. 2007, 19,51 .

31. Drain, C. M.; Varotto, A.; Radivojevic, I. Chem. Rev. 2009, 109, 1630.

32. (a) Khan, F.; Sundararajan, P.R. Org. Electron. 2006, 7, 410. (b) Janeczek, H.; Turska, E. Polymer 1987, 28, 847.

33. Bergesen, K.; Haugland, L.; , Cook, M. J.; Bryant, G. C. J. Porphyrins Phthalocyanines 2000, 4, 665 .

34. Cook, M. J.; Cracknell, S. J.; Moore, G. R.; Osborne, M. J.; Williamson, D. J. Magn. Reson. Chem. 1991, 29, 1053.

35. Eddleston, M. D.: Jones, W. Cryst. Growth Design 2010, 10, 365. 


\section{Chapter VII}

\section{Morphology of a Hydrogen Bond Mediated Self- Assembling Small Molecule in a Polycarbonate Matrix}

Presented in honour of Professor R. H. Marchessualt, who was a mentor for PRS and a collaborator for over 25 years.

Islam, M. R.; Sundararajan, P. R. Can. J. Chem. 2008, 86, 600-607. 


\subsection{Introduction}

Photoactive and electro-active functional small molecules are widely used in optoelectronic devices as a dispersed phase in polymer matrices. ${ }^{1}$ Molecular dispersion of such molecules, without phase separation is a requirement for the longevity of the device. Polycarbonate is often the preferred polymer for these applications. Thermally induced crystallization of the small molecule is a common cause of defects in e.g., photoreceptors $^{2}$ and the delamination of OLED devices. ${ }^{3}$ Abrasion is also a cause of such crystallization in photoreceptors. In a series of papers, we used the thermal annealing as a means of testing the accelerated ageing and phase separation in model composites related to photoreceptors. ${ }^{2,4,5}$

The commonly used functional small molecules in opto-electronic devices, such as the $N, N /$-diphenyl- $N, N /$-bis(3-methylphenyl)-[1,1/-biphenyl]-4,4/-diamine (TBD), tritolyl amine (TTA) etc, are non-planar and do not have segments which are associated with the term "self assembly". They do not have hydrogen bonding or extended $\pi$ stacking interactions although our studies showed some weak molecular interaction in terms of phenyl group overlap between the polycarbonate and these small molecules ${ }^{2}$. When phase separation occurs, the small molecules diffuse to the surface of the polymer film and crystallize, forming single crystals of various shapes and habits. ${ }^{2,4,5}$

Design of supramolecular structures using the self-assembling moieties is an active area at this time. Hydrogen bond, $\pi$ - stacking, and charge transfer are the common elements used for such design. It was also found to be advantageous to use multiple 
hydrogen bonding sites, using A-D-A and D-A-D groups, to control the extended assembly such as building a transient polymer based on non-covalent interactions. ${ }^{6,7}$ The nature of the side chain also plays a significant role. In such cases, the crystallization and morphology would depend on the number of such sites. In a recent study, we showed that significant differences are found in the crystallization rate, spherulite growth, etc, between molecules with single or double hydrogen bonding motifs as well as the length of the side chain. ${ }^{8,9}$ We used carbamates (one hydrogen bonding $\mathrm{N}-\mathrm{H}$.....C $=\mathrm{O}$ group, asymmetric alkyl side chain lengths) and biscarbamates (two hydrogen bonds and symmetric side chains) to this end (Fig. 7.1). These materials are candidates for the ink vehicle for phase change ink jet printing. ${ }^{10}$

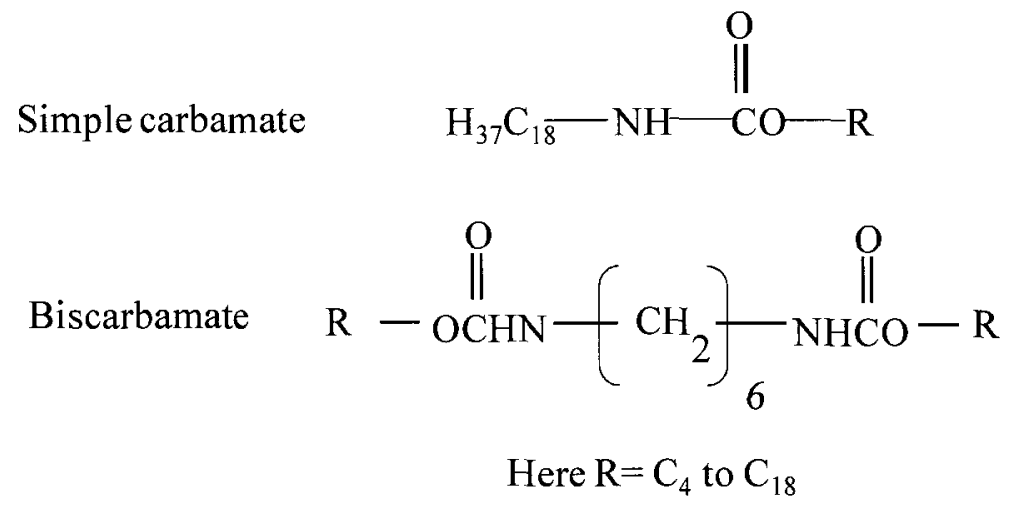

Figure 7.1: The structures of simple and biscarbamates.

Given that self assembly means spontaneous association of the molecules facilitated by the types of interactions mentioned above, it would be of interest to examine the nature of such assembly when such small molecules are dispersed in a polymer matrix. Essentially, they are confined in a polymer matrix. Although the field of polymer dispersed liquid crystals is mature, we are not referring to this case. The 
molecules under discussion are not liquid crystalline. We found that when simple carbamates were dispersed in the polycarbonate matrix, they formed colloidal size domains, of uniform size, with the domain size increasing with the relative concentration of the small molecule in the polymer. ${ }^{11}$ It was also noted that these domains form in the bulk of the polymer and not the surface. This would indicate that the self assembly and crystallization occurs before the molecules diffuse to the surface.

As we found ${ }^{8,9}$ significant differences in the crystallization features between the simple carbamates (with a single hydrogen bond) and the biscarbamates (with double hydrogen bonds), we now examine the nature of assembly when these biscarbamates are dispersed in the polycarbonate matrix. We used biscarbamates with alkyl side chain length varying from $\mathrm{C}_{4}$ to $\mathrm{C}_{18}$. Note that the concept of dispersion of self assembling small molecules in a polymer matrix is new.

\subsection{Experimental}

\subsubsection{Materials}

Poly (bisphenol-A-carbonate) $\left(\mathrm{M}_{\mathrm{w}}: 64,000, \mathrm{GPC}\right)$ and chloroform were purchased from Aldrich Chemical Company Inc. The synthesis of the biscarbamates has been described before. ${ }^{9}$

\subsubsection{Making of Films}

Generally, a 3 wt. \% of $\mathrm{C}_{4}, \mathrm{C}_{6}, \mathrm{C}_{8}, \mathrm{C}_{12}, \mathrm{C}_{16}$, or $\mathrm{C}_{18}$ biscarbamate in polymer were used. Solutions were prepared by mixing appropriate amount of the biscarbamate and 
polycarbonate in chloroform by an electrical shaker for $24 \mathrm{~h}$. Films were coated on a glass substrate using an electrically driven film coater and dried at a very low rate of solvent evaporation at ambient condition for $24 \mathrm{~h}$, and then under vacuum for $48 \mathrm{~h}$. This coater was built by Xerox Research Centre of Canada using a motor from Bodine Electric Company, Chicago, USA. The films were made using a spreader made by P. G. \& T Company. This is the same apparatus that is used by Xerox Research Centre of Canada for fabricating model photoreceptors. A set of films were also dried at $50^{\circ} \mathrm{C}$ for $48 \mathrm{hrs}$, to assess the effect of temperature. The thickness of the films was about 10-15 $\mu \mathrm{m}$. This film thickness was used for all of our previous work on model charge transport layers of photoreceptors and verified by SEM of cross sections. ${ }^{2}$ Another set of films of $\mathrm{C}_{4}$ and $\mathrm{C}_{6}$ biscarbamates with concentrations ranging from 0.75 to $10 \mathrm{wt} \%$ were also prepared.

\subsubsection{Characterization Methods}

A Zeiss Axioplan 2 imaging universal optical microscope with Q-imaging Retiga digital camera was used to record optical micrographs of the films. In addition, a JSM6400 scanning electron microscope was also used. The surface of the films was coated with a conductive layer for taking the SEM images.

The Fourier transform infrared (FTIR) spectroscopic measurements were performed at ambient conditions using a Michelson M120 BOMEM FTIR spectrometer. The BOMEM GRAMS/386 software was used for data collection. The films were attached to the holder by a small magnet and placed to the path of radiation and the spectra were recorded. 


\subsection{Results and Discussion}

\subsubsection{Polarizing Optical Microscopy}

The solutions and the films were transparent for the case of $\mathrm{C}_{4}$ and $\mathrm{C}_{6}$. The biscarbamates used here do not exhibit specific interactions with polycarbonate. In the infrared spectra, we did not observe any absorption peak for the as-prepared films that could be identified with non-hydrogen bonded $\mathrm{C}=\mathrm{O}$ and $\mathrm{N}-\mathrm{H}$ groups of the biscarbamates, although the N-H group showed a peak at $3314 \mathrm{~cm}^{-1}$ instead of the usual $3337 \mathrm{~cm}^{-1}$ that is assigned to the hydrogen bonded group. Such a shift just confirms the strength of the interactions between the biscarbamates while in the polymer matrix. Fig 7.2 shows the optical micrographs of the polycarbonate films containing the various biscarbamates. With $\mathrm{C}_{4}$ and $\mathrm{C}_{6}$, the self assembling small molecules form spherical domains, which almost look like a donut. They aggregate to form a meandering chain. The images are not sharp, as it appears that they are covered with a layer of the polymer. Note that polycarbonate itself is amorphous in this case. This is in contrast to the phase separation and crystallization of other small molecules from the polymer matrix, where the crystals forms on the surface of the polymer film. These may be compared with distinct crystals of the charge transport molecules TBD and TTA shown in Fig. 6 of Ref 2(b), Fig 3 of Ref 4, and Fig 11 of Ref 5. As the alkyl chain length increases, the crystals begin to appear, radiating from nodes (as shown by the arrows). It appears that these nodes are protruding crystals that have formed during the diffusion of the small molecule from the bulk (Fig 7.2 for $\mathrm{C}_{12}$ and $\mathrm{Cl}_{16}$ ), but the crystallization had already taken place before the completion of the diffusion step. 

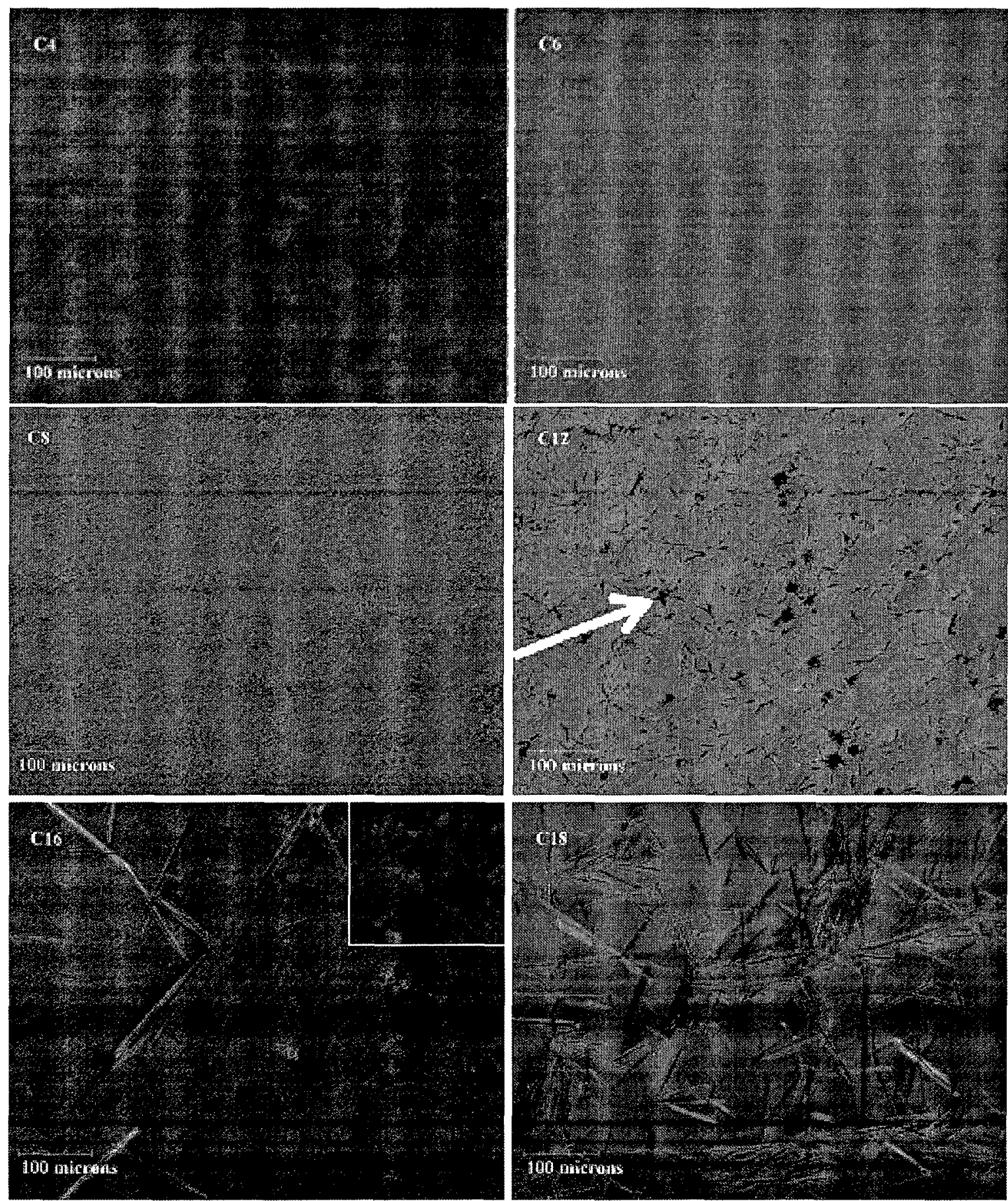

$100 \mu m$

Figure 7.2: Optical micrographs of polycarbonate films containing (3 wt \%) biscarbamates with different alkyl chain lengths.

One of these areas is shown as an inset for $\mathrm{C}_{16}$ which shows radiating lamellae from a protrusion. We have shown before ${ }^{9}$ that the rate of spherulite growth is a 
maximum for $\mathrm{C}_{8}$, and that of $\mathrm{C}_{12}$ is faster than for $\mathrm{C} 4$. Although long needles of crystals are seen in for the case of $\mathrm{C}_{16}$, there are a number of areas which show a bulge and radiating lamellae. It is due to these bulges that we infer that the growth has already taken place before the diffusion of the molecules to the surface.

Similar to the case of polycarbonate/ simple carbamates system ${ }^{11}$, we see a competition between diffusion of the small molecule from the matrix to the surface and the crystallization in the bulk of the polymer before the diffusion is complete. The rate of crystallization of the simple carbamates by themselves was very fast that we could not study their rates of spherulite formation etc. ${ }^{8}$ However, we were able to measure such rates with biscarbamates. ${ }^{9}$ As discussed before, the rate of self assembly depends on the length of the alkyl chains and it was seen that the alkyl chains stabilize the structure. ${ }^{9}$ It was shown using IR spectra that the packing of the alkyl chains is enhanced with the chain length. In the present case, with the longer alkyl chain lengths, we see some needlelike crystals on the surface as well as circular asperities that indicate crystals growing in the bulk. As we mentioned before, when small molecules crystallize out of the polymer, they would tend to form crystals of various types of habits that depend on the growth mechanism. In this case the asperities as well as the lamellae radiating from the nodes indicate an irregular spherulite formation. Thus, the faster growth rate of the biscarbamates with shorter alkyl chains result in crystallization below the surface. As the alkyl chain length increases and the growth rate decreases, crystals appear on the surface, as seen in other cases (as in Ref 2,4 and 5). Fig. 7.3 shows the micrographs of some of 
the samples that were dried at $50^{\circ} \mathrm{C}$. The morphologies for $\mathrm{C}_{4}, \mathrm{C}_{6}$ and $\mathrm{C}_{12}$ are similar to those seen in Fig. 7.2.
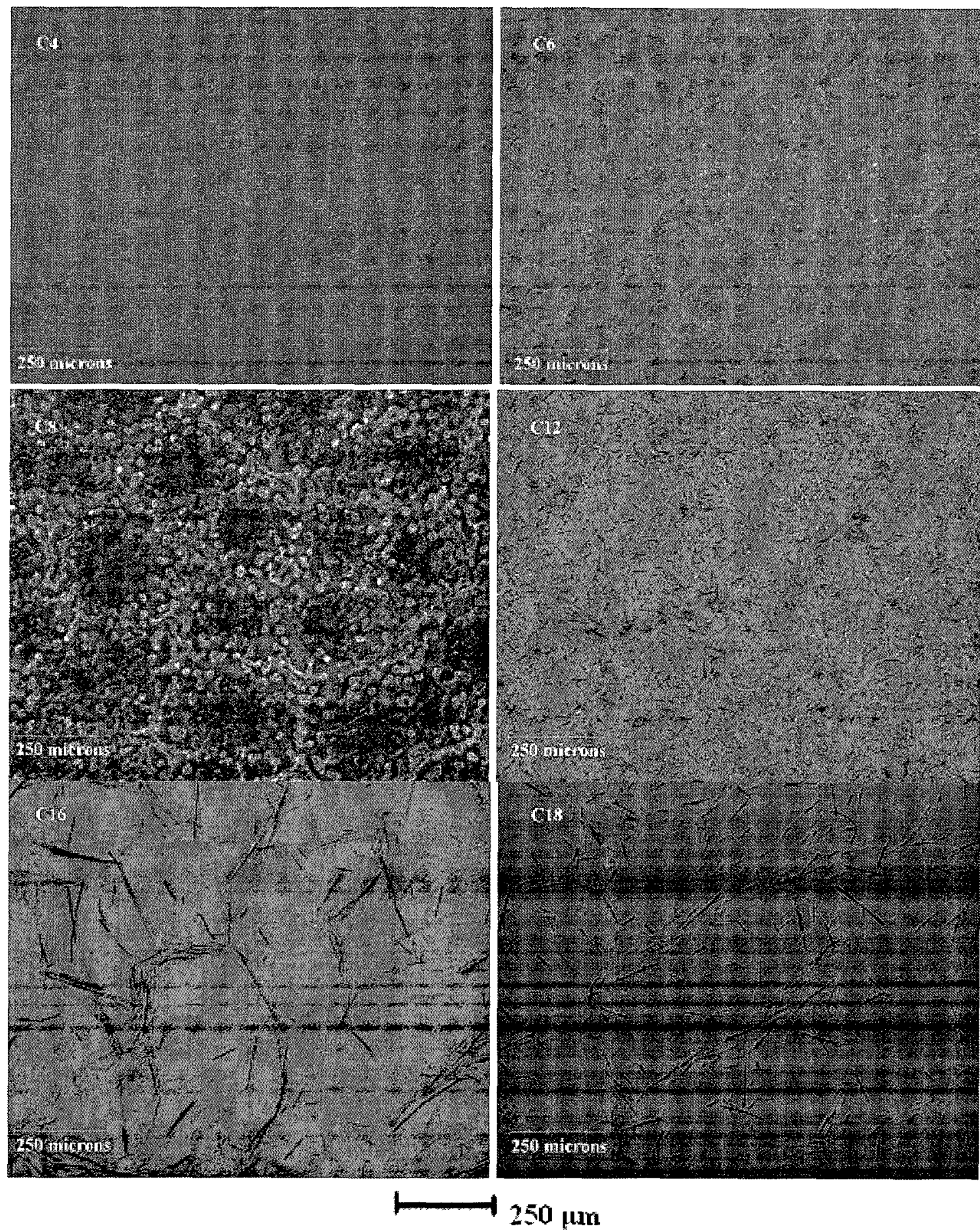

Figure 7.3: Optical micrographs of films that were dried at $50^{\circ} \mathrm{C}$. 
For $\mathrm{C}_{8}$, we observe features similar to those of $\mathrm{C}_{6}$. Spherical patches are also seen. Perhaps due to the enhanced diffusion to the surface at the higher temperature, $\mathrm{C}_{16}$ and $\mathrm{C}_{18}$ show crystals on the surface, and the bumps or nodes that were seen in Figure 7.2 are not present. Thus the morphology for the biscarbamates with longer alkyl chains differs with this mild thermal treatment. We did not examine this aspect in detail at this time.

The effect of concentration on the domains is shown in Fig. 7.4 for $\mathrm{C}_{4}$ biscarbamate. With a concentration of $2 \%$, the morphology is similar to what is shown in Fig. 7.2. With 4\%, "patchy" regions appear which increase in number with concentration. There are the crystalline domains that formed in the bulk of the polymer film. These domains are not clearly seen since these are covered by a layer of the polymer, which is amorphous. However, the polymer film itself shows strain birefringence around these patchy domains. With a high concentration of $10 \%$, dense lamellar growth is seen. 

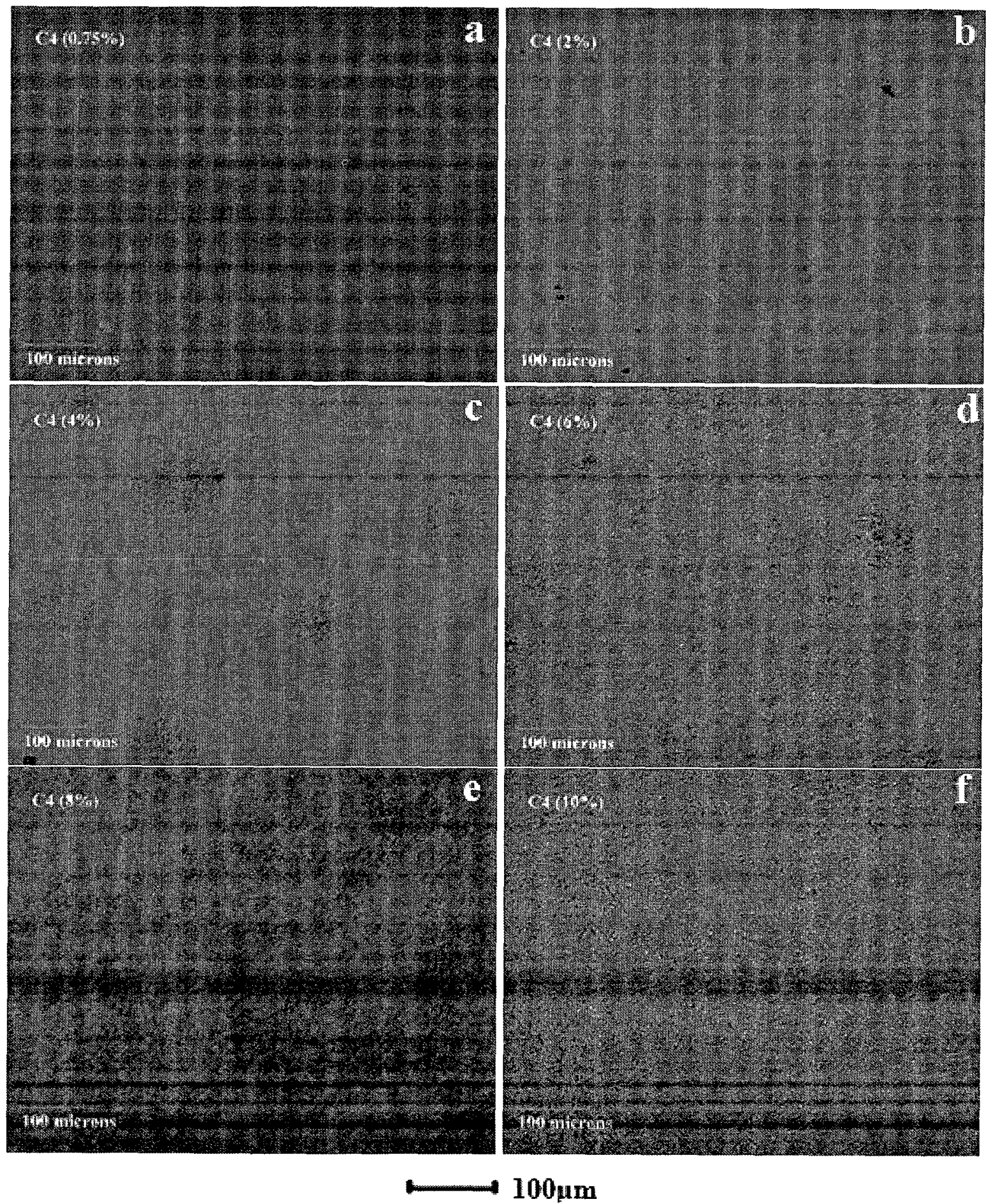

Figure 7.4: Optical micrographs of polycarbonate films with different concentrations of $\mathrm{C}_{4}$ :

(a) $0.75 \%$ (b) $2 \%$ (c) $4 \%$ (d) $6 \%$ (e) $8 \%$ and (f) $10 \%$ respectively. 


\subsubsection{Scanning Electron Microscopy}

The SEM images shown in Fig. 7.5 illustrate the sub-surface growth of the crystalline domains of the biscarbamates. The image for $\mathrm{C}_{4}$ shows the enlarged view of the morphology of the "donuts" seen in Fig 7.2.
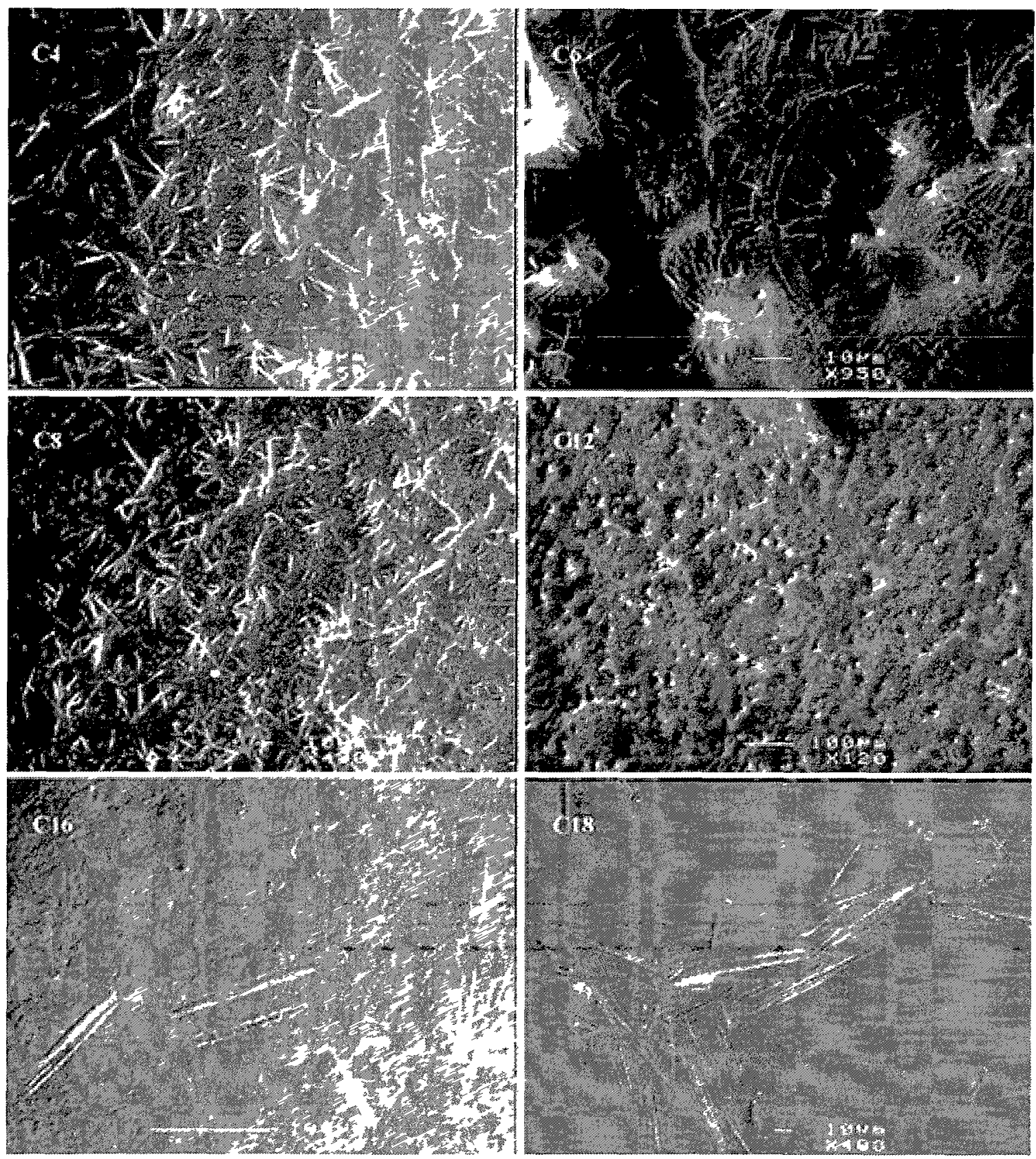

Figure 7.5: SEM images of the films made with different biscarbamates. 
It is seen that the domains appear as if covered by a layer of the polymer. This is quite pronounced in the case of $\mathrm{C}_{12}$. Even with $\mathrm{C}_{16}$, the crystalline lamellae are not well above on the surface of the film. Thus, the self assembly and the fast rate of crystallization give rise to sub-surface morphologies. As we investigate this phenomenon further, we plan to study the effect of the thickness of the film and the SEM images of cross sections.

\subsubsection{Thermal Reversibility}

With the polycarbonate/ simple carbamate system we found that the domains can be melted and reformed. The $\mathrm{T}_{\mathrm{m}}$ of the simple carbamates vary from 61.2 to $86.5^{\circ} \mathrm{C}$ as the alkyl chain length increases from $\mathrm{C}_{4}$ to $\mathrm{C}_{18}$. The $\mathrm{T}_{\mathrm{m}}$ of the biscarbamates are higher, ranging from 90 to $120{ }^{\circ} \mathrm{C} .{ }^{9}$ Fig. 7.6 shows the micrographs of the polycarbonate/ biscarbamate system, which was held at the melting temperature of each of the biscarbamates for two minutes and cooled by taking off from the hot plate (all the images on the left side in Fig. 7.6). Another set of samples was held at the $T_{m}$ for 30 minutes and cooled.

The samples that were kept for 2 minutes at the $T_{m}$ show the morphological features similar to those seen in Figure 7.2, but become more diffuse. The blur in the images is due to the covering of the polymer layer on the small molecule crystalline domains. The protrusions that were seen for $\mathrm{C}_{16}$ in Figure 7.2 are seen here for the case of $\mathrm{C}_{18}$. The process of melting and cooling did not result in the crystallization on the surface. We mentioned in the introduction that the biscarbamates are candidates as 
vehicles for phase change inkjet inks. Their melt viscosity is only a few centipoises, in the range of $8-12 \mathrm{cp}$.
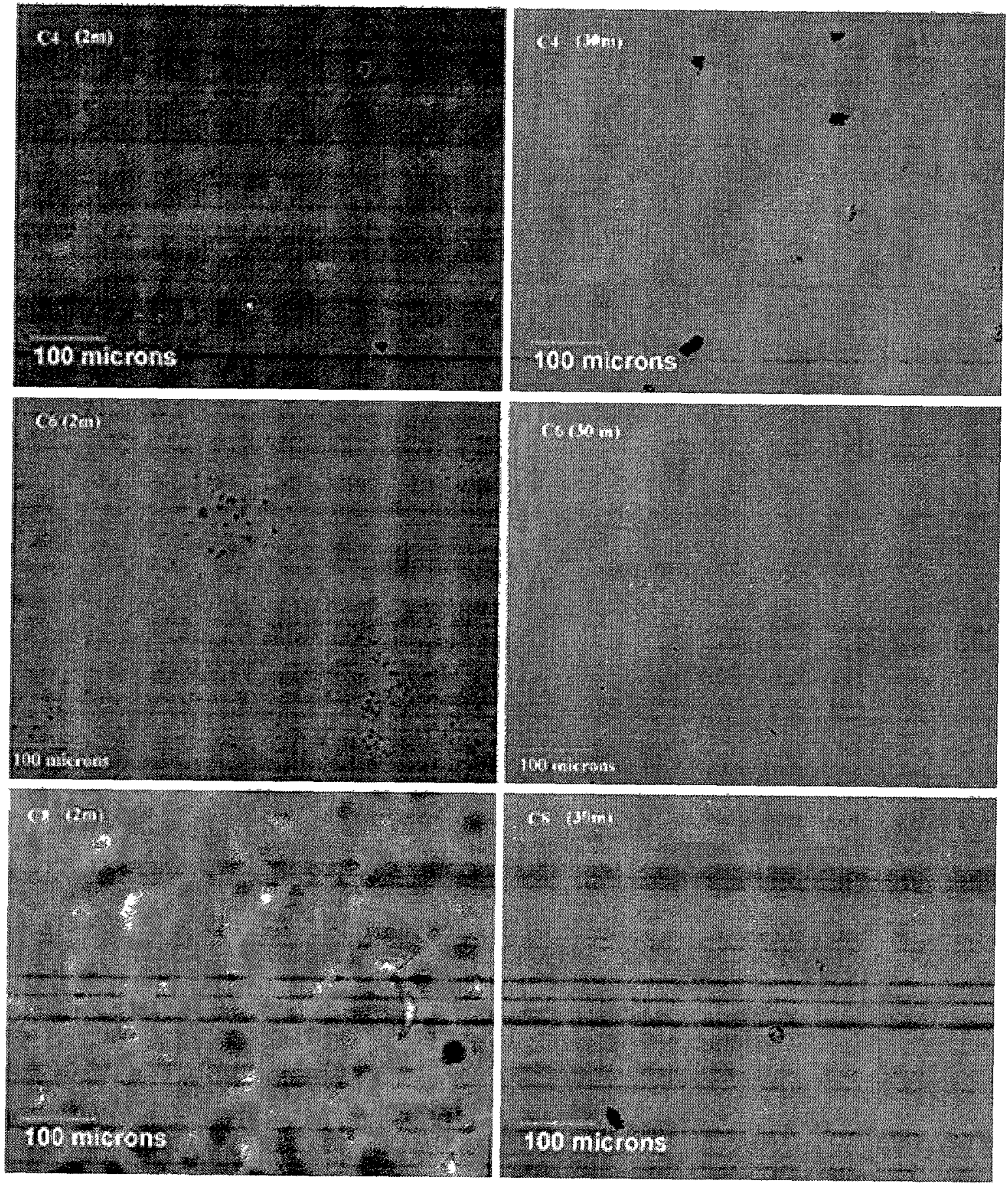

Figures continued in the next page 

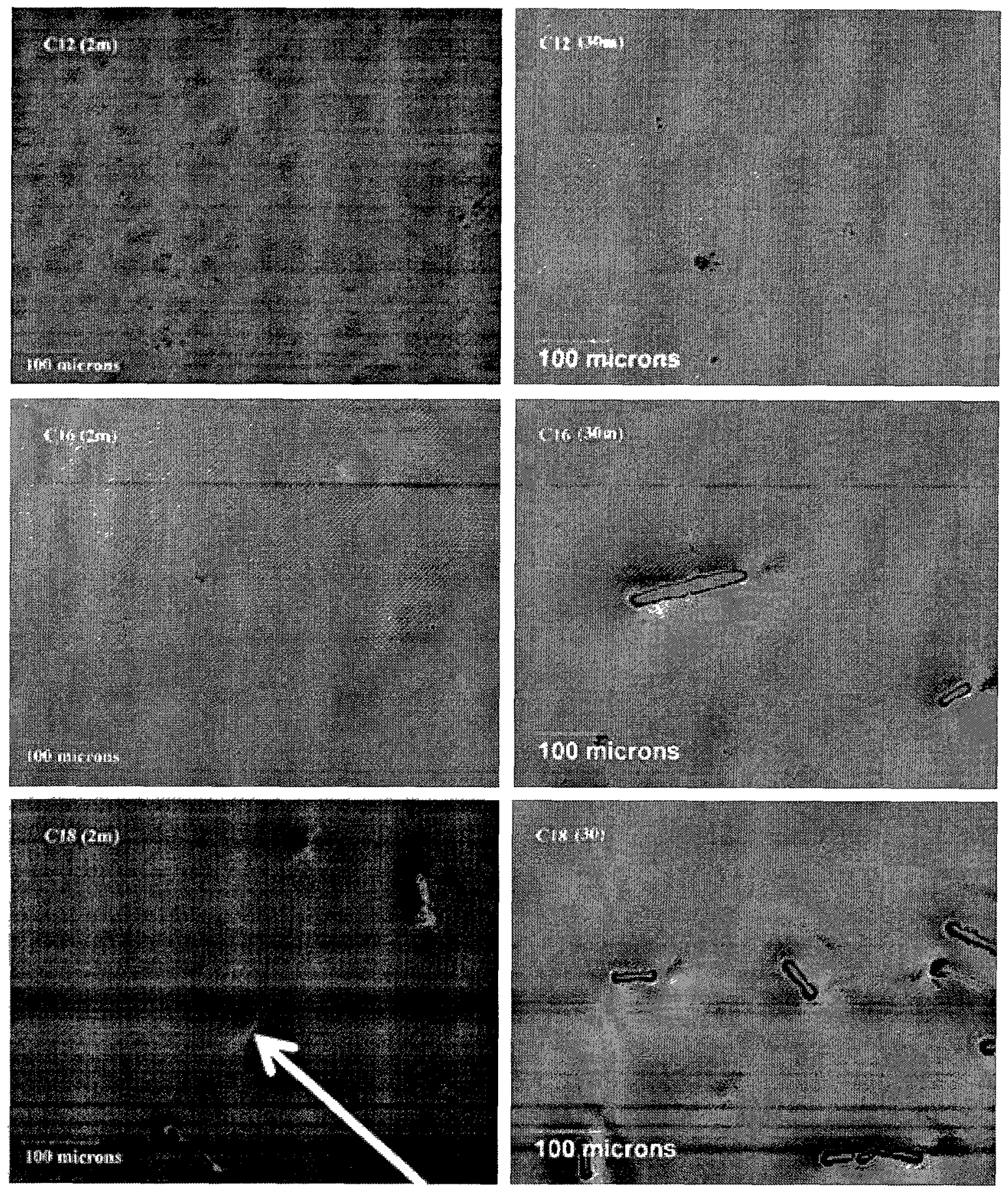

Figure 7.6: Optical micrographs of the films heated to the melting temperature of the respective biscarbamate and cooled. The images for samples that were kept at $T_{m}$ for 2 minutes (left) and 30 minutes (right) are shown. 
The images in Fig. 7.6 for the samples kept at $T_{m}$ for two minutes indicate that melt of the biscarbamates remained distributed in the bulk, and did not form droplets on the surface. Thus the interfacial tension between the polycarbonate and the melt of biscarbamate has a significant effect on the morphology, due to the low melt viscosity of the small molecule. Thus, by heating the composite to the $T_{m}$ of the biscarbamates and cooling within a short time creates sub-surface crystallization.

This effect is amplified when the samples are kept at the $T_{m}$ for 30 minutes. All the images in Fig. 7.6 for this case do not show any features. Even with $\mathrm{C}_{16}$ and $\mathrm{C}_{18}$, no crystals are observed. It appears that during the $30 \mathrm{~min}$, the melt diffused into the polycarbonate matrix and the crystals did not form again. In the FTIR spectra of these samples the peaks corresponding to the hydrogen bonded $\mathrm{N}-\mathrm{H}$ and $\mathrm{C}=\mathrm{O}$ groups (at about 3315 and $1684 \mathrm{~cm}^{-1}$ respectively) were not seen. Instead, a broad peak at about 3450 was seen for the non-hydrogen bonded N-H group. Thus, the 30 minute dwell time and subsequent cooling created a molecular dispersion of the biscarbamate in polycarbonate, with no intermolecular hydrogen bonded interaction between these molecules, which have the self-assembly motif in their structure.

\subsection{Conclusions}

The morphologies of self-assembling molecules in polymer matrices are different from those of molecules which do not possess self-assembling codes (hydrogen bond, $\pi$ interaction etc), as seen from this work as well as our previous study on a related system. ${ }^{11}$ As the small molecules would normally diffuse to the surface of the polymer 
film and crystallize, we propose that the rate of crystallization due to self assembly is faster than the diffusion to the surface. This is the cause of the sub-surface morphology that was discussed above. The biscarbamates used here do not exhibit specific interactions with polycarbonate. In the infrared spectra, we did not observe any absorption peak for the as-prepared films that could be identified with non-hydrogen bonded $\mathrm{C}=\mathrm{O}$ and $\mathrm{N}-\mathrm{H}$ groups of the biscarbamates, although the $\mathrm{N}-\mathrm{H}$ group showed a peak at $3314 \mathrm{~cm}^{-1}$ instead of the usual $3337 \mathrm{~cm}^{-1}$ that is assigned to the hydrogen bonded group. Such a shift just confirms the strength of the interactions between the biscarbamates while in the polymer matrix. There was no shift in the $\mathrm{C}=\mathrm{O}$ peak of the polycarbonate $\left(1175 \mathrm{~cm}^{-1}\right)$. The $\mathrm{Tg}$ of the polycarbonate was reduced to about $125^{\circ} \mathrm{C}$ with $\mathrm{C}_{4}$ and $\mathrm{C}_{6}$ biscarbamates ( $3 \mathrm{wt} \%$ ), but no noticeable change with the longer alkyl side chains.

The longer dwell time of the films at the $T_{m}$ of the biscarbamates results in the absence of IR peaks due to hydrogen bonded $\mathrm{N}-\mathrm{H}$ and $\mathrm{C}=\mathrm{O}$ groups which indicates the molecular dispersion of the small molecules and the lack of any association between them. Thus the behavior of self-assembling molecules in a polymer matrix is distinctly different from that of other cases, e.g., the composites of charge transport layers of optoelectronic devices, with functional photoactive molecules dispersed in a polycarbonate matrix. 


\subsection{References}

1. (a) Borsenberger, P. M.; Weiss, D. S. Organic Photoreceptors for Xerography, Marcel

Decker. New York, 1998. (b) Melnyk, A. R.; Pai, D. M.; Hard Copy and Printing Materials, Media and Process, Proc. SPIE, 1990. 1253, 141.

2. (a) Khan. F.; Hor, A. M.; Sundararajan. P. R. J. Phys. Chem. 2004, 108. 117. (b) Khan, F.; Sundararajan, P. R. Org. Electronic 2006, 7, 410.

3.Smith. P. F.; Gerroir, P.; Xie. S.; Hor, A. M.; Popovic, Z.: Hair, M. L. Langmuir 1998, 14, 5946 .

4. Khan, F.; Hor, A. M.; Sundararajan, P. R. Pure Appl Chem. 2004. 76. 1467.

5. Khan, F.: Hor, A-M.; Sundararajan, P. R. Synthetic Metals 2005,150, 199.

6. Lehn. J. M. "Supramolecular Chemistry", VCH: Germany, 1995.

7. Ikeda, M.; Nobori, T.; Schmutz, M.; Lehn, J. M. Chem. Eur. J. 2005, 11, 662.

8. Moniruzzaman, M.; Goodbrand. B.; Sundararajan, P. R. J. Phys. Chem. Part B 2003, 107. 8416 .

9. Khanna, S.: Moniruzzaman. M.; Sundararajan, P.R J. Phys. Chem Part B 2006. 110, 15251.

10. Goodbrand, B.; Boils, D. C.; Sundararajan, P. R.; Wong, R. W. US Patent, 2002, 6414-051. 
11. Tuteja, B.; Moniruzzaman, M.; Sundararajan, P. R. Langmuir, 2007, 23, 4709. 


\section{Chapter VIII}

\section{Conclusions \& Recommendations}




\subsection{Conclusions}

The purpose of the thesis was to study the self-assembly in solution and solid state of some functional organic molecules with $\pi$ - or $\mathrm{H}$ - bonding moiety in a confined state from a morphological point of view. Controlled morphology is a very active area of research due to its immense possibility of creating diverse and new applications or applications with controlled and enhanced properties. We know the potential of perylene and phthalocyanine in optoelectronic device fabrication, solar cell and biological imaging due to their extended $\pi$-system, fluorescent ability, semiconductor property and high thermal and photolytic stability. If we can achieve the control over morphology of these functional molecules in a confined state by attaching or dispersing them to polymer matrix of different nature we would be able to tune the properties as well. We have developed methodologies to control the morphologies in solution or in solid state thin films of functional molecule with $\pi$ - and H-bonding moiety attached or dispersed into a polymer matrix. This study of self assembly of small functional molecules in polymer matrix will help us control the properties through morphology in optoelctronic devices.

Our initial aim was set to prepare soluble perylene dimide block copolymers to study their self assembly and morphology in solution. In line with that a photoconducting block, perylene was attached with hydrophilic and hydrophobic polymer covalently. We have found that the self-assembly can be induced and controlled by concentration and solvent systems. We have achieved control over the extent of aggregation, stability and morphology of perylene based pseudo block copolymers either in solution or in the thin film solid state. We have also achieved the control over phase 
separated morphology of a $\pi$-bonding molecule, substituted phthalocyanine and a series of molecules with two H-bonding moieties, biscarbamates in polycarbonate and PMMA matrices. This study of self assembly in a confined state will widen our knowledge to control the properties of functional molecules through morphologies. In the following paragraphs we will see how the control over self assembly in a confined state was achieved.

We have prepared water soluble rod-coil and coil-rod-coil polymers, with hydrophilic Jeffamine ${ }^{\mathbb{B}}$ as the flexible segment and photo-conducting large aromatic perylenediimide (PTCDI) as the rigid rod. Jeffamine was attached either to one side of PTCDI (MJ-PTCDI) and the other side with isobutyl group or both sides (DJ-PTCDI). Because of the hydrophilic nature of Jeffamine, PTCDI forms $\pi$-interaction mediated aggregates in aqueous solution and these are H-stacked in MJ-PTCDI and J-stacked in DJ-PTCDI. Variable temperature UV and NMR spectra show that the assembly is stable over a large temperature range in water. The extent of aggregation and hence the mole fraction of aggregates can be controlled in a mixture of solvents. The optical microscopy of the bulk material shows aggregated crystals of PTCDI in the waxy matrix while drop cast films from aqueous solution give spherical morphology. With ageing of the aqueous solutions, formation of a fiber morphology was seen, which is believed to be formed by the coalescence of spherical aggregates one-dimensionally. Although UV-Vis spectra indicated no aggregation in non-aqueous solvents, drop-cast films of these solutions show needle-like aggregates and lego-like assemblies. In addition to this rod-coil block copolymer we have prepared a series of block copolymers (PJ-PTCDI, H-JPTCDI and 
OJ-PTCDI) where one side of perylene is attached with Jeffamine and the other side with linear alkyl chain of different lengths. We find that the morphology of this type of block copolymer can be controlled from bundle of fibers to transparent sheets and tubes by increasing the alkyl chain length in aqueous solution. Other than water they self assemble from different non aqueous solvents during evaporation and form spherical. needle or fibrous aggregates. The fiber aggregates of OJ-PTCDI show potential to form water soluble gels. Due to the solubility in water and good fluorescence of these block copolymers; they are interesting material for use as fluorescence probes in biological applications.

In an effort to study the self-assembly of perylene diimide in nonaqueous solvent by attaching short chain low molecular weight polystyrene to both sides of perylene (PSPTCDI-PS) we have shown that the morphology of the aggregate can be controlled to porous sheet or nano sphere in solid state. Although, this does not show any aggregate in non-solvent solutions with concentration, the extent of self-assembly could be controlled by a judicious choice of mixture of solvent systems like acetone-water, chloroformmethanol and acetone-methanol etc. The mode of aggregation can be controlled either to $\mathrm{H}$ - type or J-type providing a route to control the direction of electron flow in optoelectronic devices.

We have reported that the self-assembly of a substituted Octabutoxy phthalocyanine $(\mathrm{Pc})$ in polymer matrices leads to tubular morphology when the film is prepared with tetrachloroethane (TCE) and sub-surface droplet morphology with chloroform. The same morphology is seen with both bisphenol-A polycarbonate 
(BPAPC) and poly (methyl methacrylate) (PMMA) as the matrix. The sub-surface morphology results from the rapid association of $\mathrm{Pc}$ in the polymer matrix, as the film forms. Even in the absence of the polymer. Pc crystallized from TCE also shows tubular morphology, while it exhibits a columnar morphology with chloroform. We attribute the difference in the morphology to the higher viscosity of TCE, which enables slow growth leading to tubular morphology, while the instantaneous self-assembly in less viscous chloroform leads to droplets. The solvent effect observed here highlights the potential fine tuning of phase separation which could be used to tailor the morphology of such photoconductive molecules in polymer matrices. The control of the morphology of small crystalline molecule in polymer films by rational choice of solvent-polymer systems can provide a range of options for fabrication of devices for photoreceptor, solar cell.

We have studied the self-assembly of a series of biscarbamates (two hydrogen bonding moiety with symmetric side chain from $\mathrm{C}_{4}$ to $\mathrm{C}_{18}$ ) in polycarbonate (BPAPC) matrix. With shorter alkyl group they form donut-like spherical morphology where-as the others with longer alkyl chains form needle like morphology. We observe a competition between diffusion of the small molecule from the matrix to the surface and the crystallization in the bulk of the polymer before the diffusion is complete. As the small molecules would normally diffuse to the surface of the polymer film and crystallize. we propose that the rate of crystallization due to self-assembly is faster than the diffusion to the surface. This is the cause of the sub-surface morphology of biscarbamates in polymer matrix, BPAPC. With higher concentration short chain biscarbamates show lamellar morphologies. 


\subsection{Recommendations for Future Work}

Our previous study shows a vesicular nano-structure with PDMS on both sides or a web morphology with PDMS attached on one side of a perylene unit. Due to the increasing demand for environmentally friendly processing and formulation, we used water soluble Jeffamine to make water soluble perylene bisimides in our present study. Perylene diimides attached with Jeffamine on one side and PDMS or polystyrene on the other side could be an interesting field of research where a balance of hydrophilic and hydrophobic polymer chain could be maintained. We have initiated this work. We have synthesized perylene diimides where Jeffamine is attached in one side of imide nitrogen and PDMS of molecular weight 875,1500 and 3035 and polystyrene $\left(M_{n}=1000\right)$ are attached on the other side. Early result shows spherical morphologies from PDMSPTCDI-Jeffamine diimides as such and in different non-solvents. The fluorescent emission color can be tuned from green to maroon to red providing a route to control the extent of aggregation in nonaqueous solvents.

A future student can work on attaching more bulky polymers with conformational restriction like PMMA. Bisphenol A Polycarbonate or an aromatic polymer on one side and both sides of perylene to study polymer confined self-assembly in aqueous-non aqueous systems.

Another avenue of future research could be to see how the self-assembly of these perylene bisimides can be controlled or varied by increasing the length of the central perylene unit. That will certainly show a bathochromic shift in the absorption spectra due 
to extended conjugation. This approach has potential for near IR absorbing sensor application where we will be able to tune the aggregate structure to absorb or emit at a very specific frequency. Interesting morphologies could be generated as well.

The study of gelation of these block copolymers of perylene diimide could be another interesting field of research. As we know most of our perylene diimides are water soluble, we will be able to create water soluble gels of perylene diimides of controlled morphologies. This work has already been started (Elianne Dahan. $2^{\text {nd }}$ year $\mathrm{PhD}$ ) in our lab. 
Appendix A

FTIR Spectra 


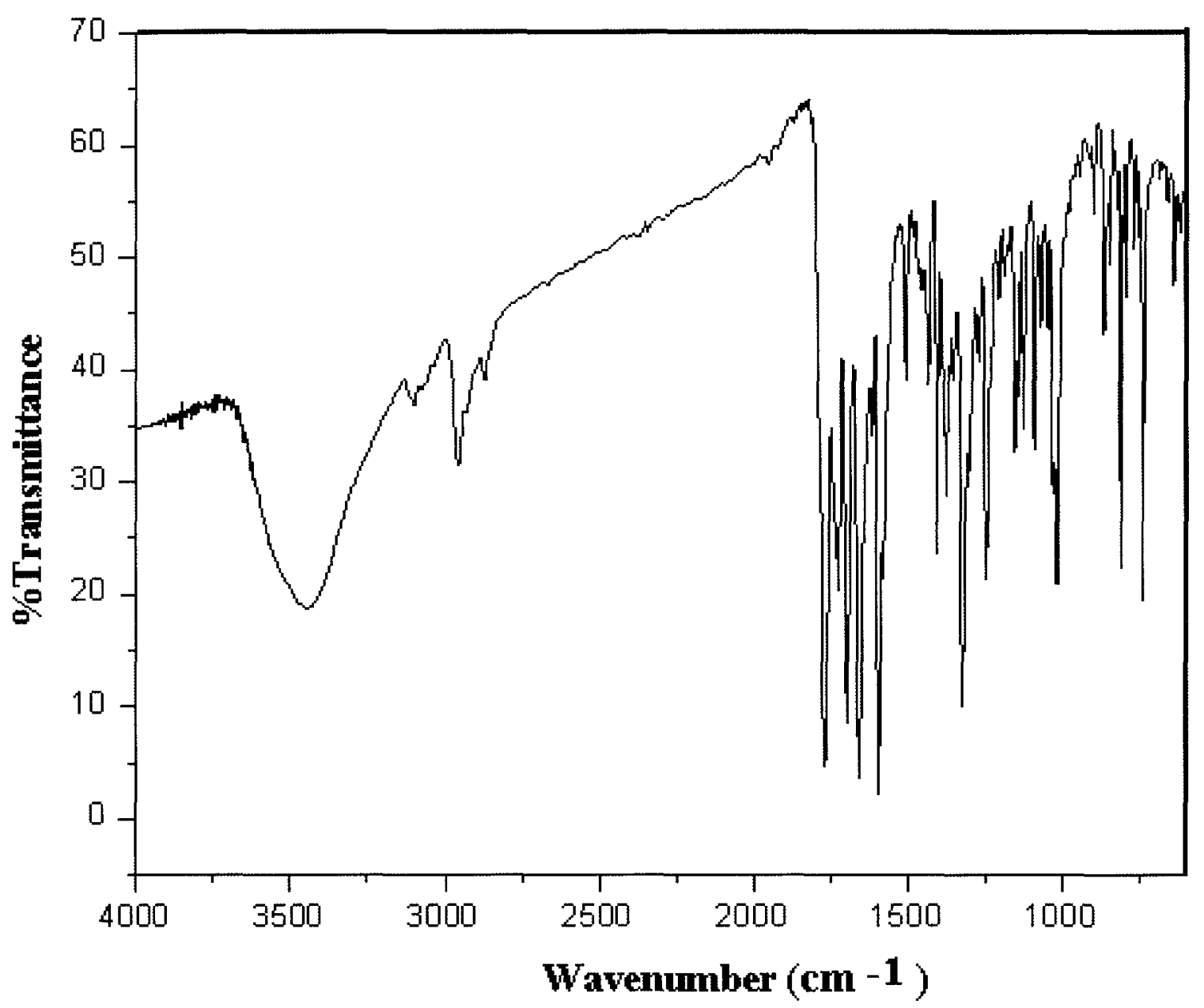

Figure A1: FTIR of N-isobutyl-3, 4, 9, 10-perylenetetracarboxylic monoanhydride monoimide. 


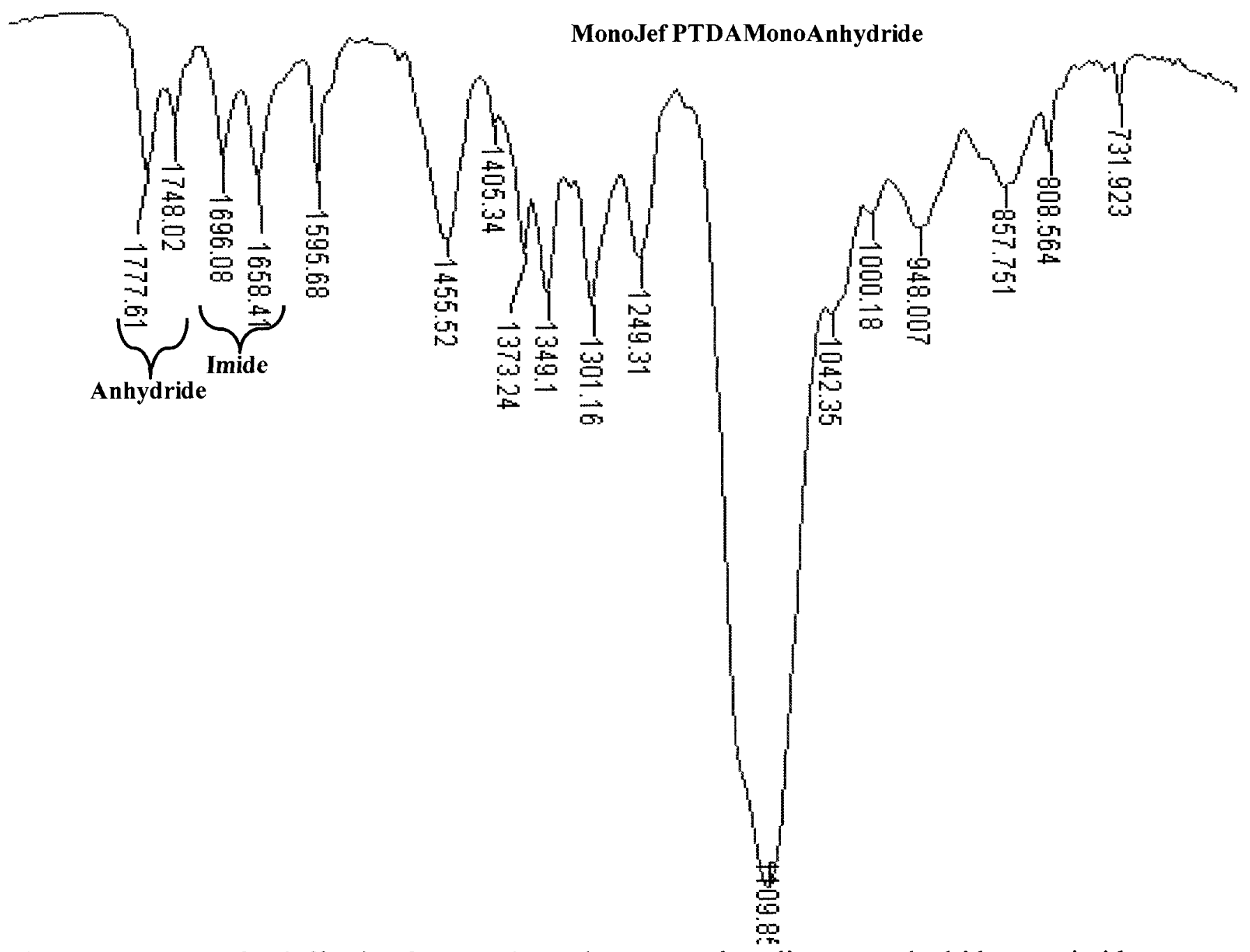

Figure A2: FTIR of N-jeffamine-3, 4, 9, 10-perylenetetracarboxylic monoanhydride monoimide. 


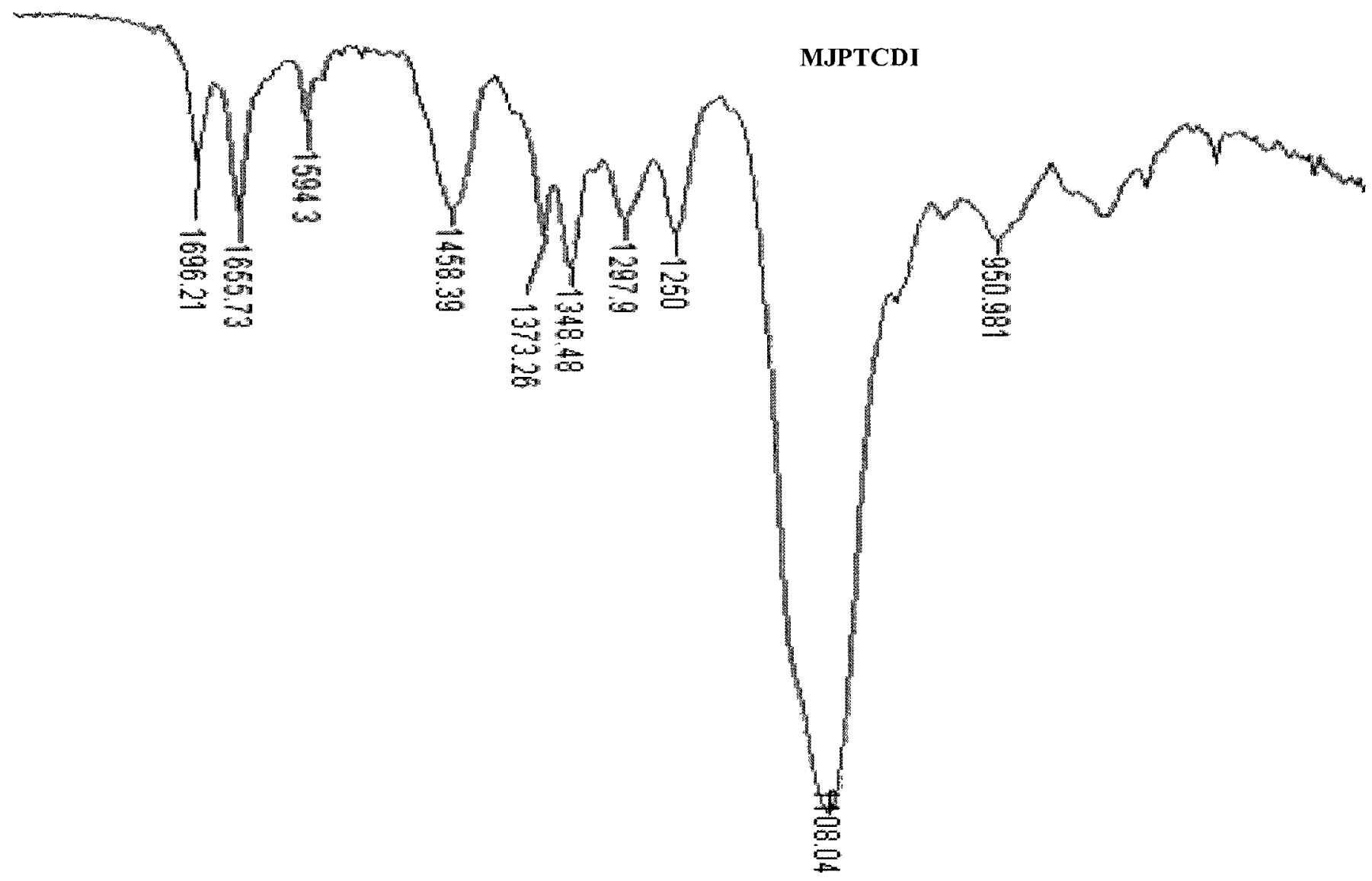

Figure A3: FTIR of MJPTCDI. 


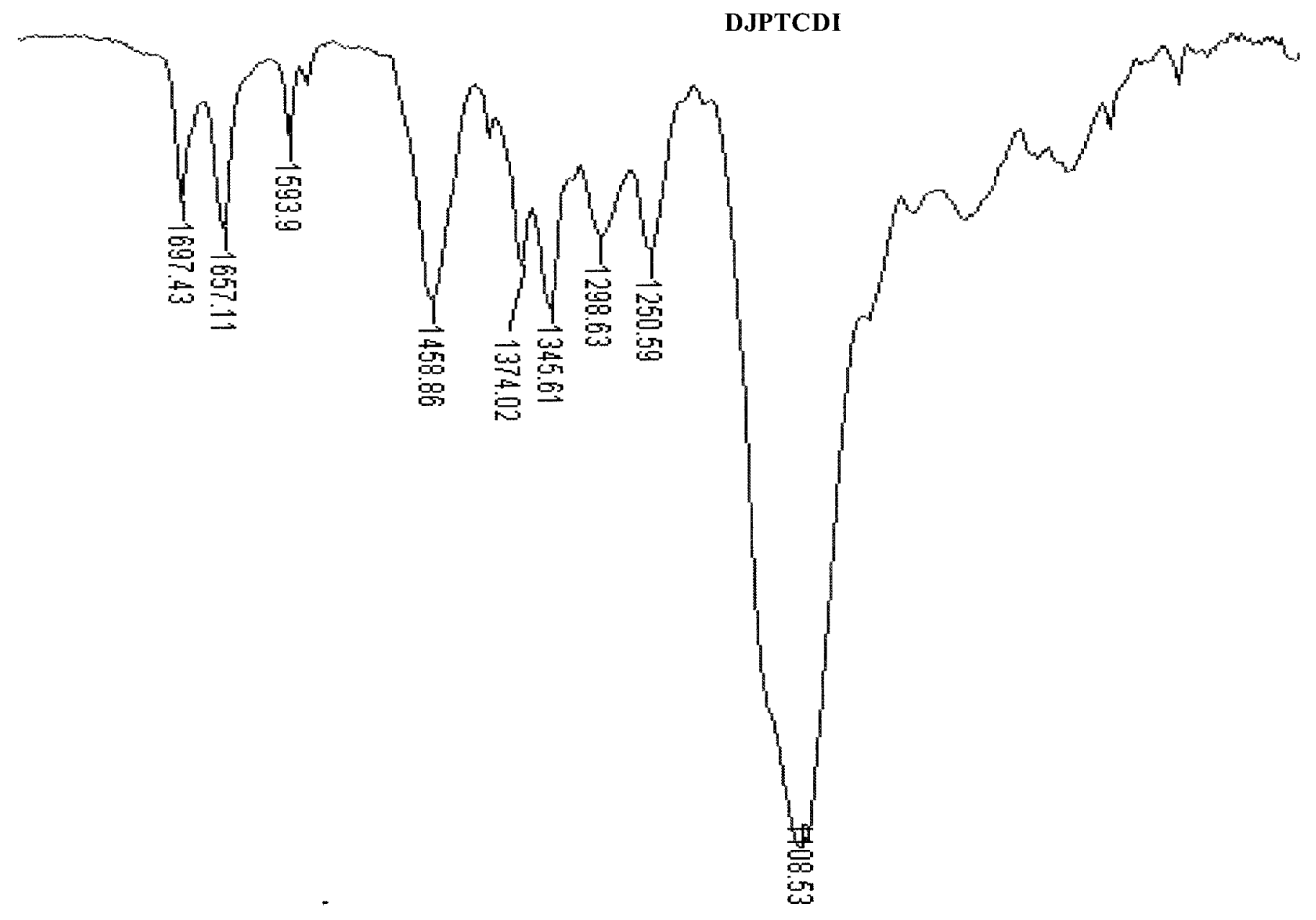

Figure A4: FTIR of DJPTCDI. 

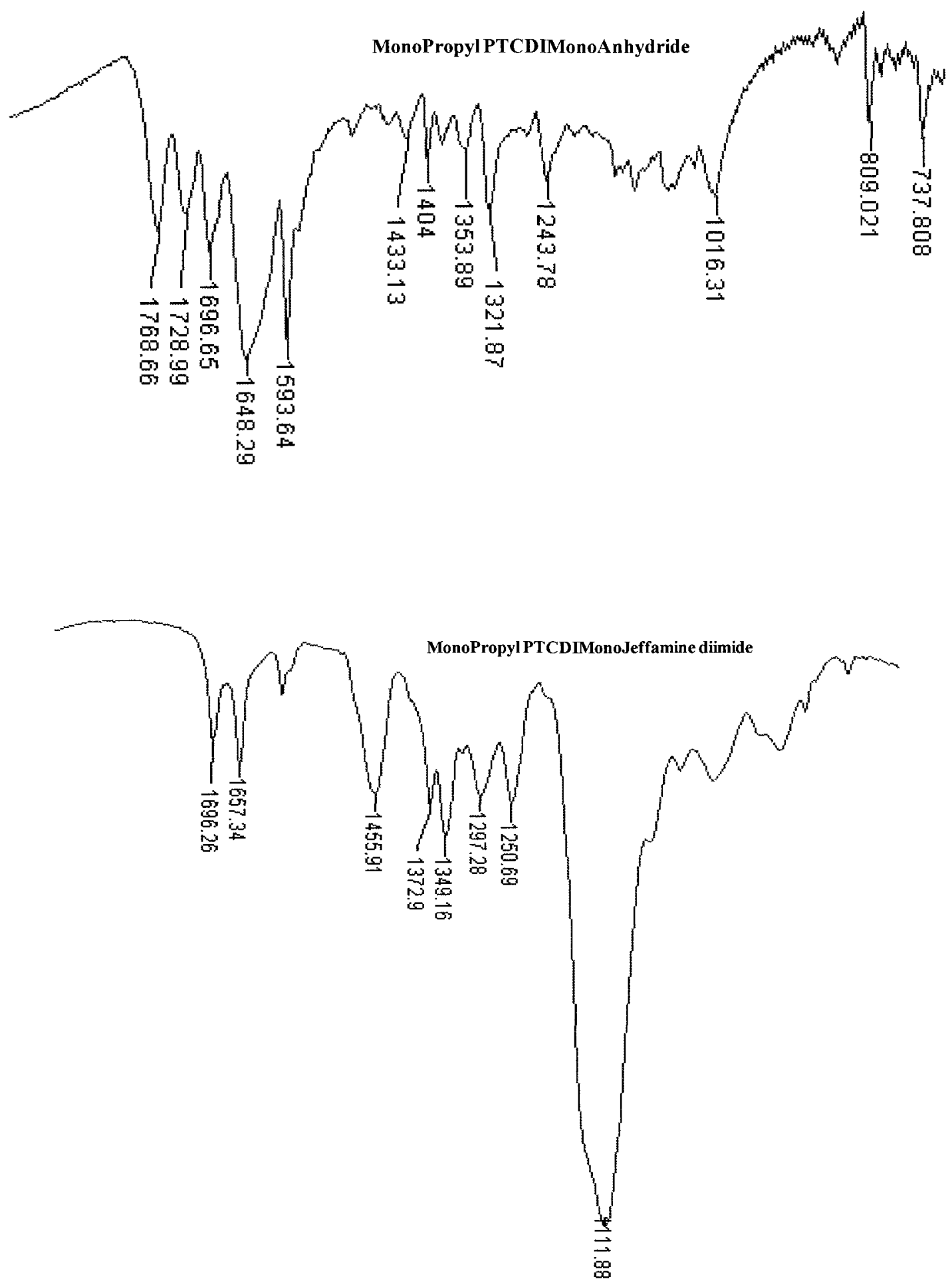

Figure A5: FTIR spectra of Monopropyl perylene monoanhydride (upper) and Monopropyl monojeffamine perylene tetracarboxylic "diimide (lower). 

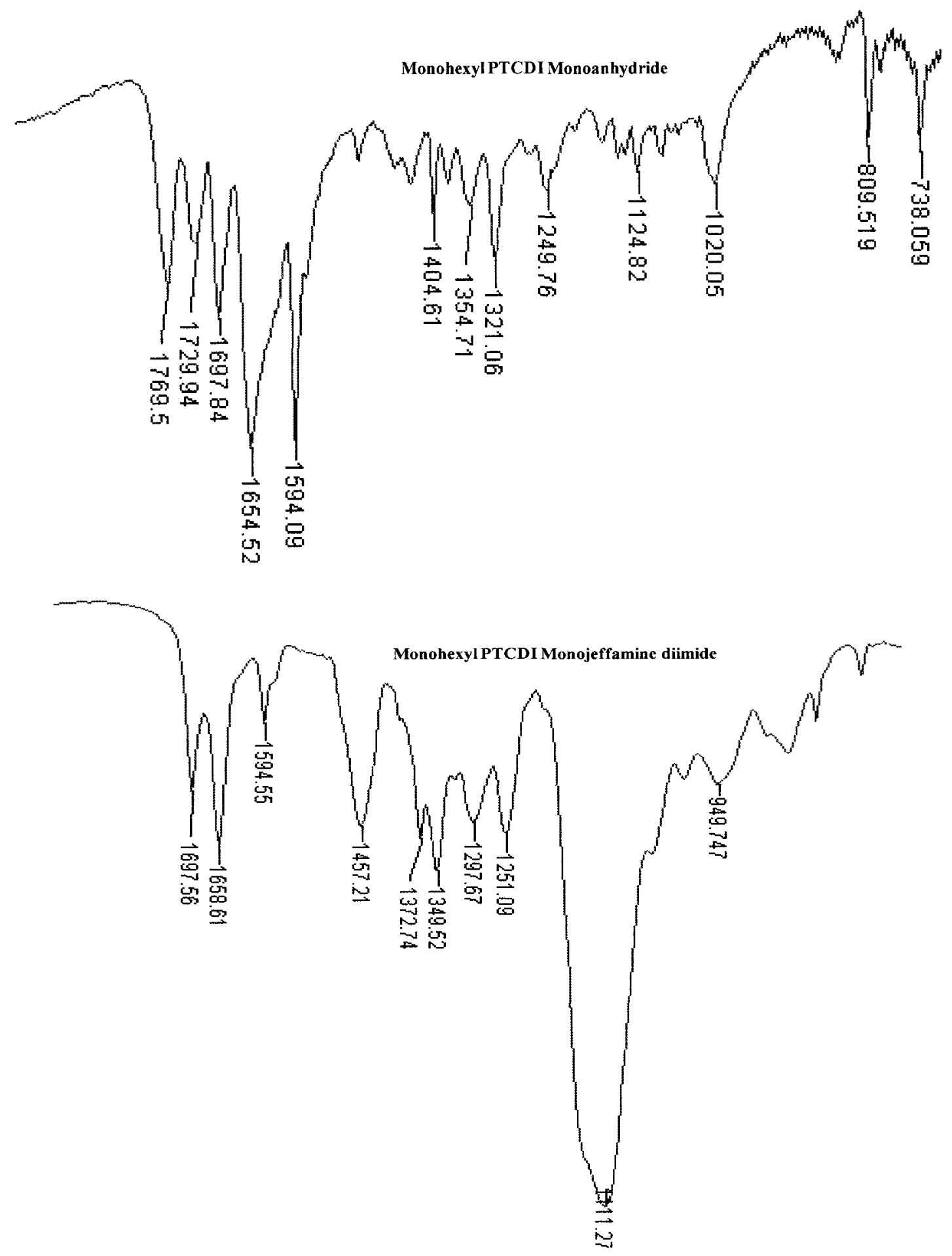

Figure A6: FTIR spectra of Monohexyl perylene monoanhydride (upper) and Monohexyl monojeffamine perylene tetracarboxylic diimide (lower). 
viii
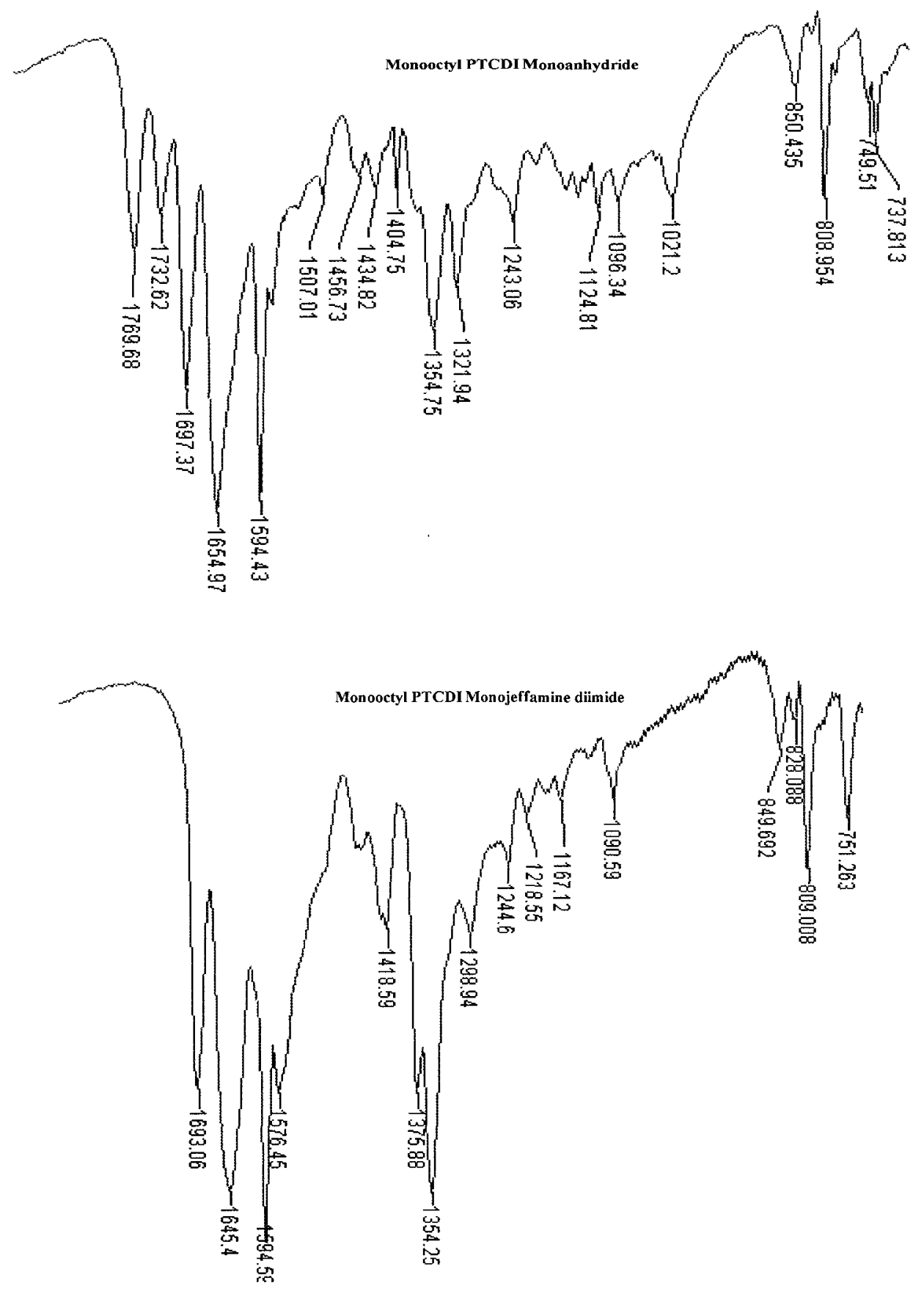

Figure A7: FTIR spectra of Monooctyl perylene monoanhydride (upper) and Monooctyl monojeffamine perylene tetracarboxylic diimide (lower). 


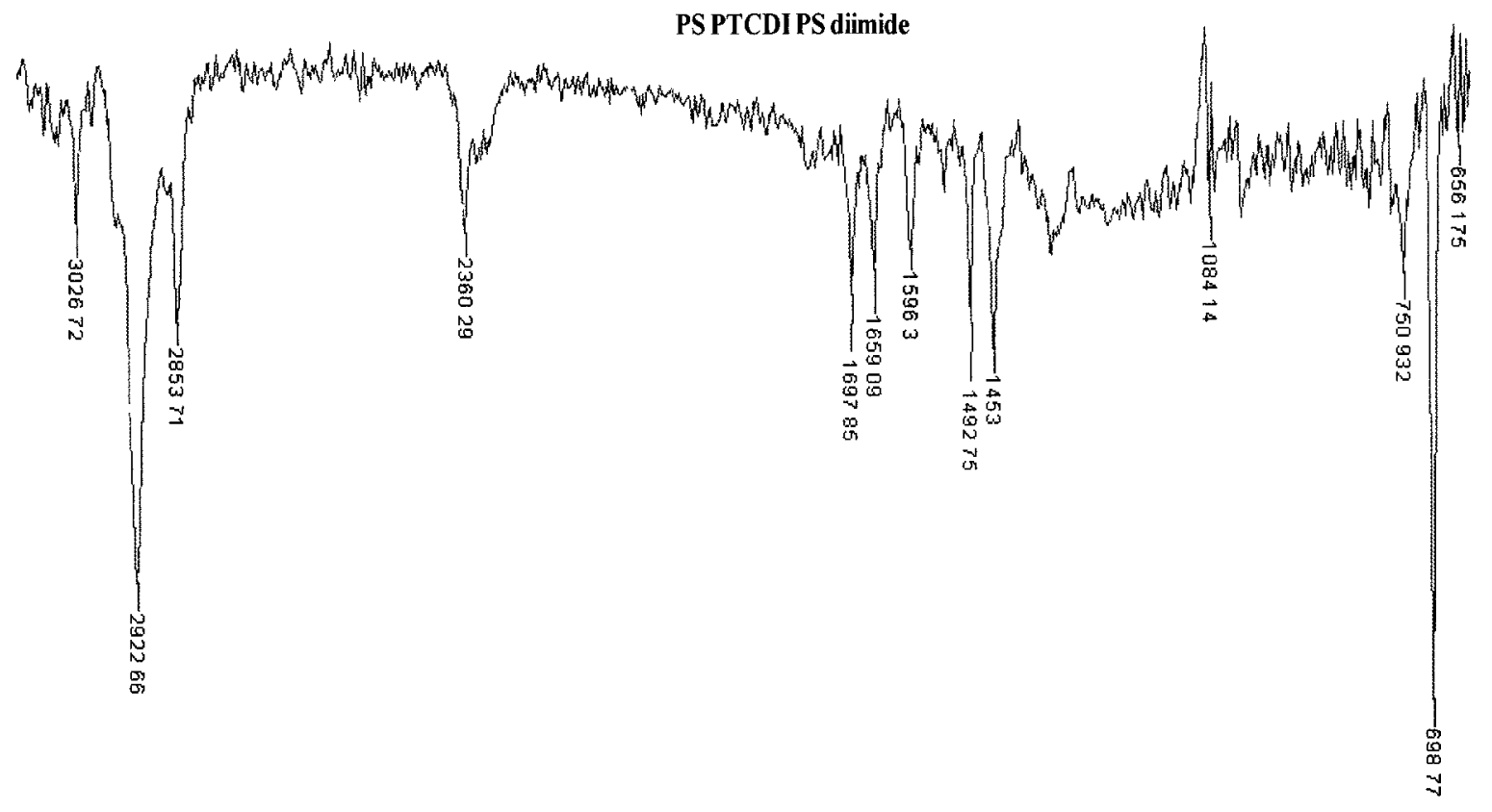

Figure A8: FTIR spectra of PS-PTCDI-PS perylene diimide. 


\section{Appendix B NMR Spectra}




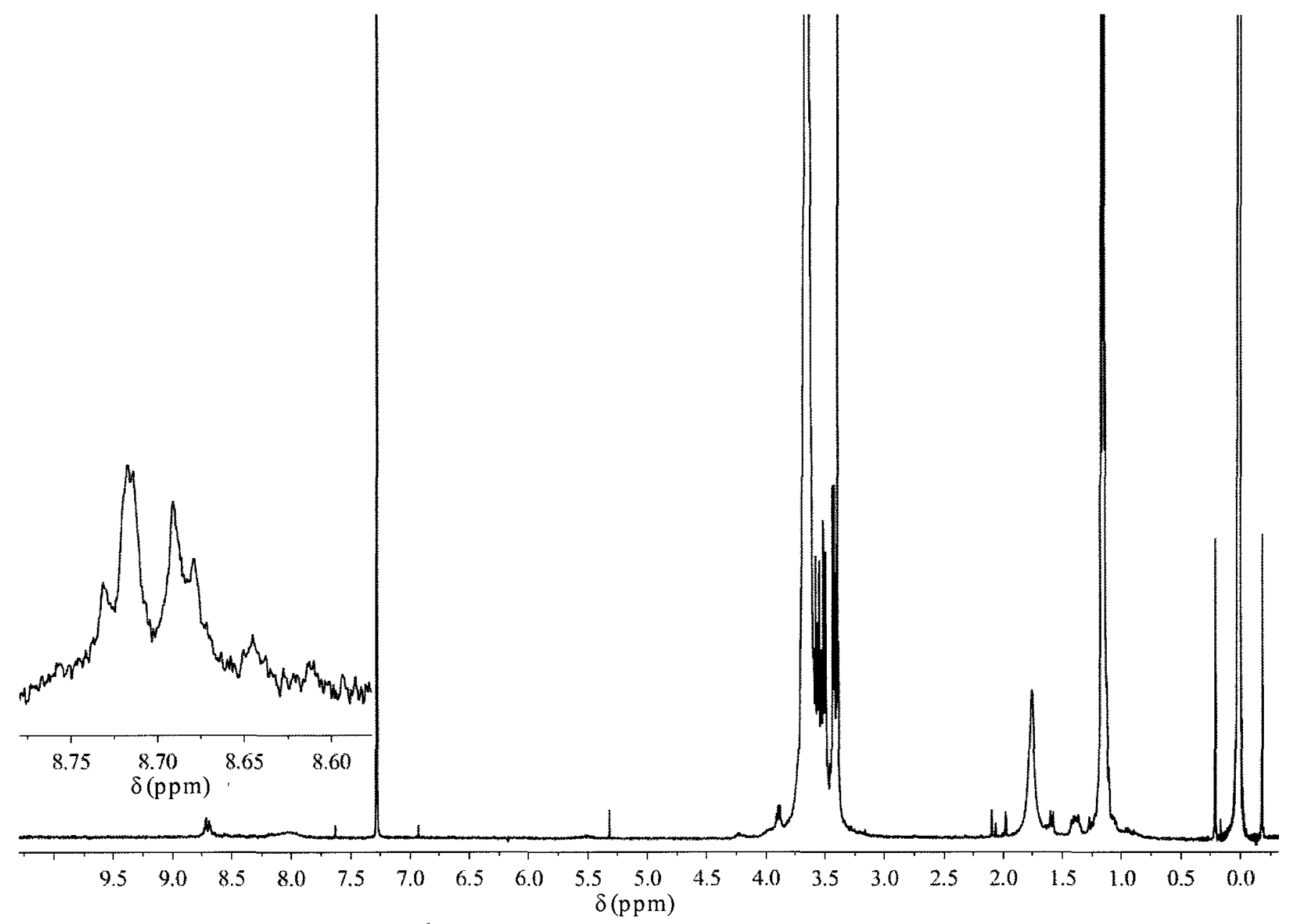

Figure B1: ${ }^{1} \mathrm{HNM}$ spectra of JeffaminePTCDImonoanhydride in $\mathrm{CDCl}_{3}$. 


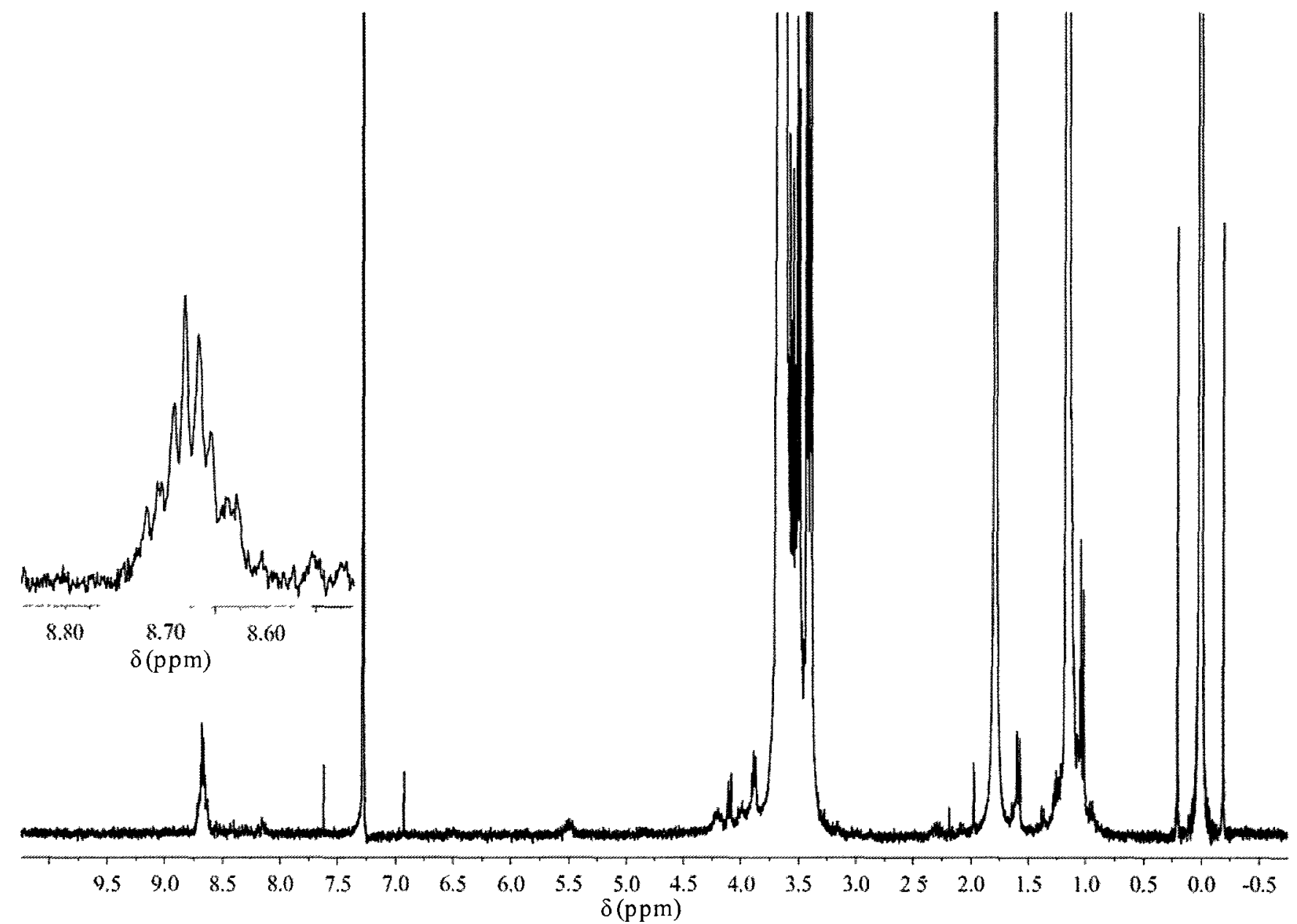

Figure B2: ${ }^{1} \mathrm{HNM}$ spectra of MJPTCDI in $\mathrm{CDCL}_{3}$ 


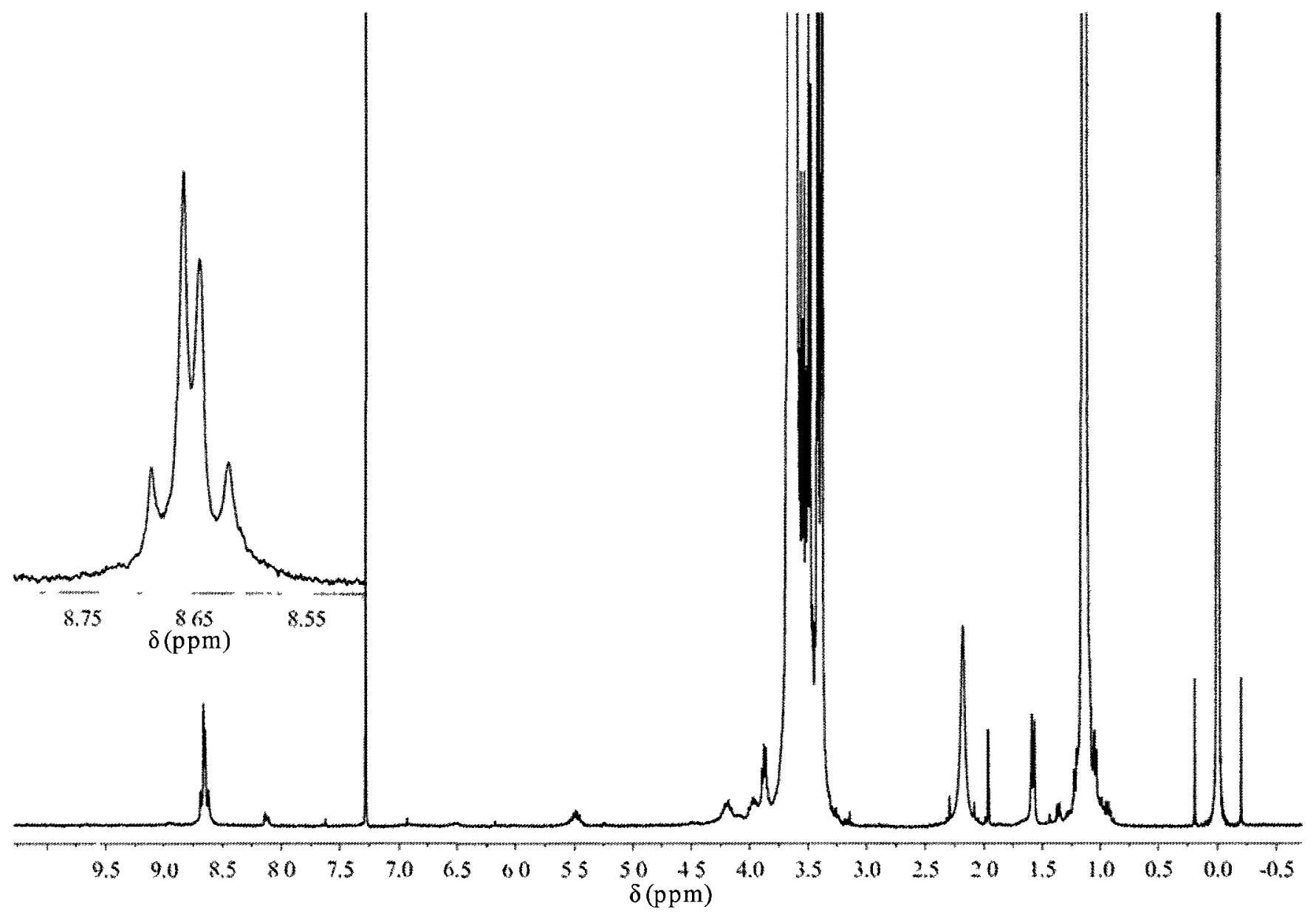

Figure B3: ${ }^{1} \mathrm{HNM}$ spectra of DJPTCDI in $\mathrm{CDCL}_{3}$ 


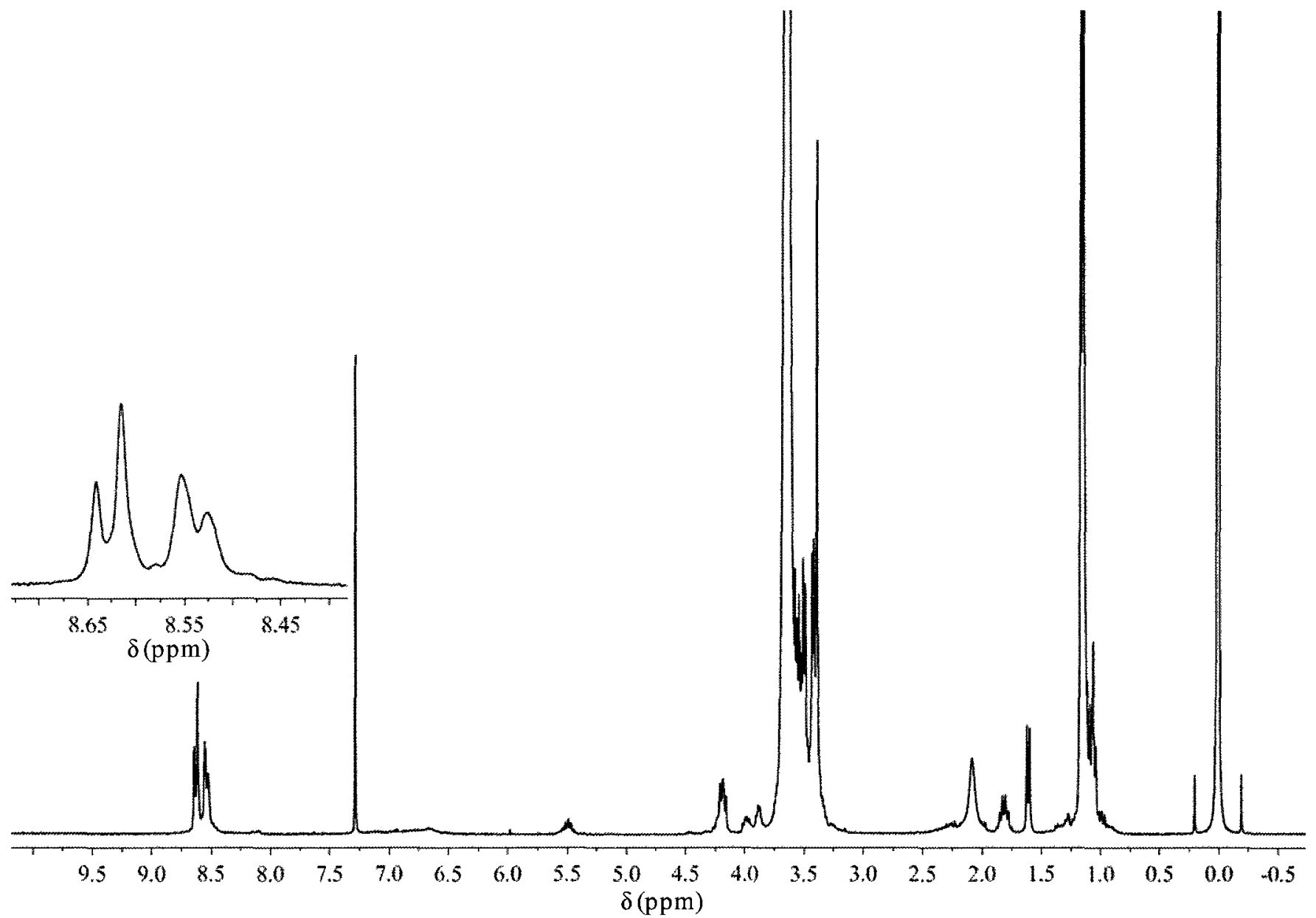

Figure B4: ${ }^{1} \mathrm{HNMR}$ spectra of PJPTCDI in $\mathrm{CDCL}_{3}$. 


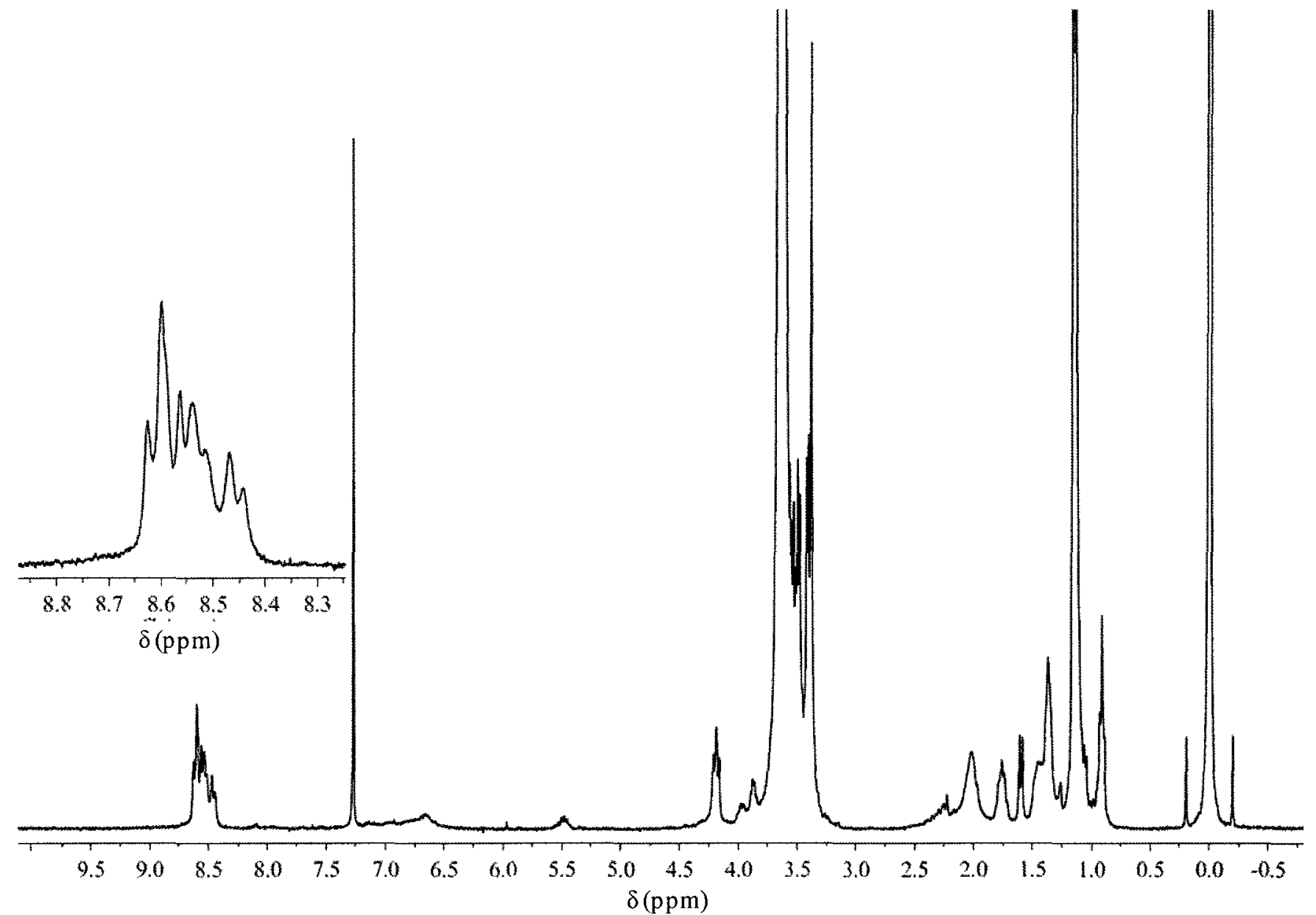

Figure B5: ${ }^{1}$ NMR spectra of HJPTCDI in CDCL $_{3}$ 


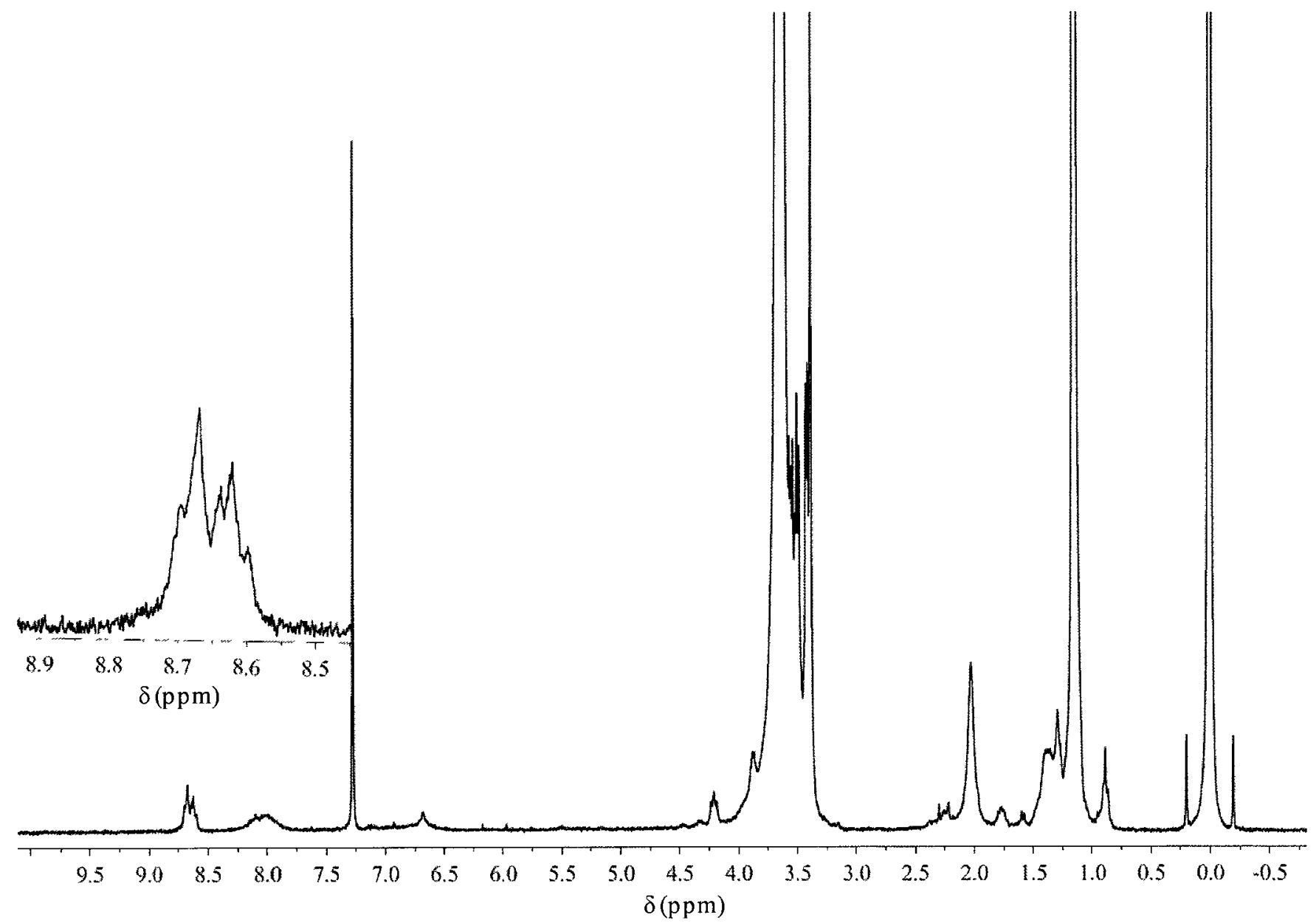

Figure B6: ${ }^{1} \mathrm{HNMR}$ spectra of OJPTCDI in $\mathrm{CDCl}_{3}$ 


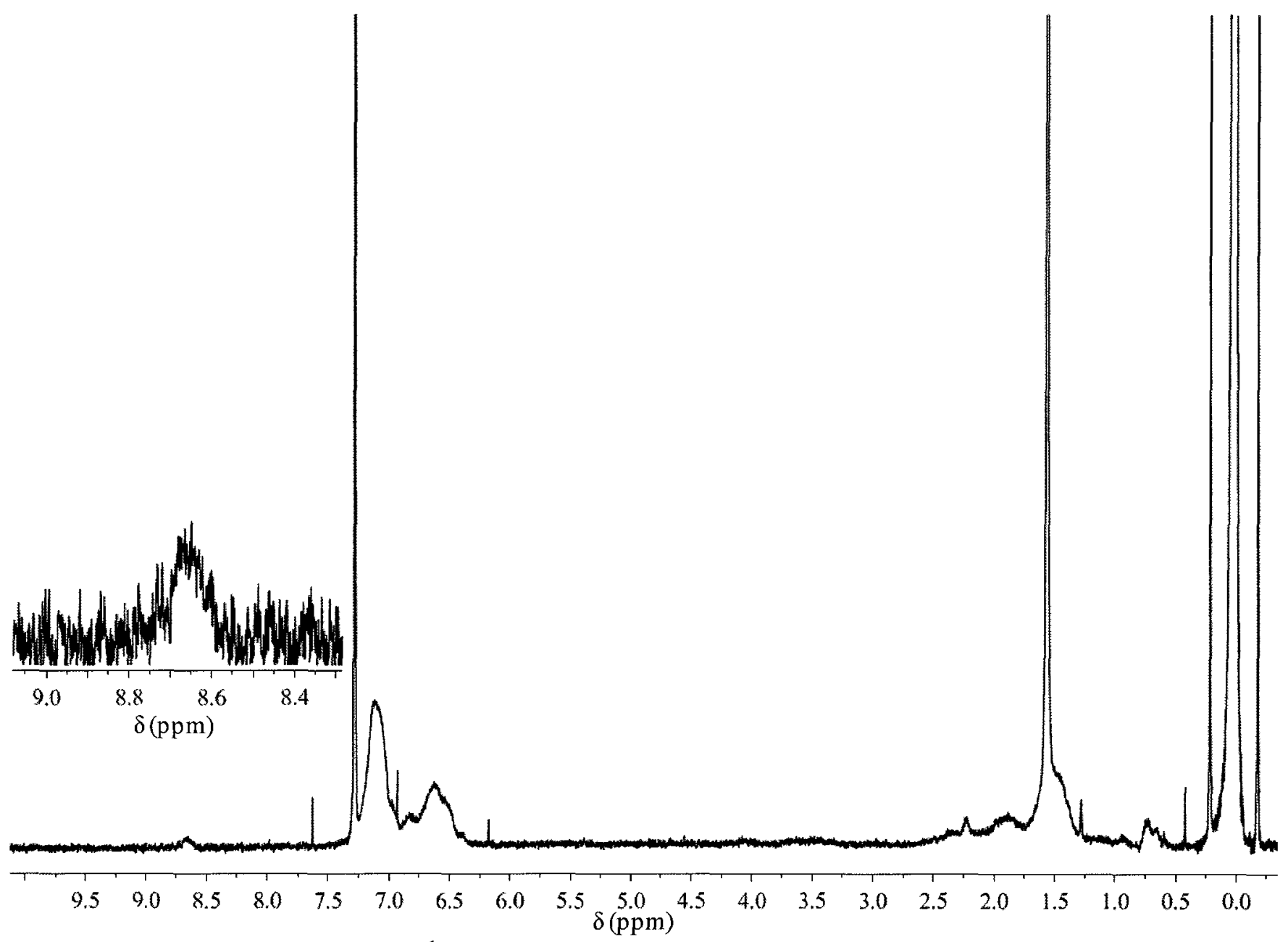

Figure B7: ${ }^{1} \mathrm{HNM}$ spectra of PSPTCDIPS in $\mathrm{CDCL}_{3}$. 


\section{$\underline{\text { Appendix C }}$}

Photographs of Solutions 

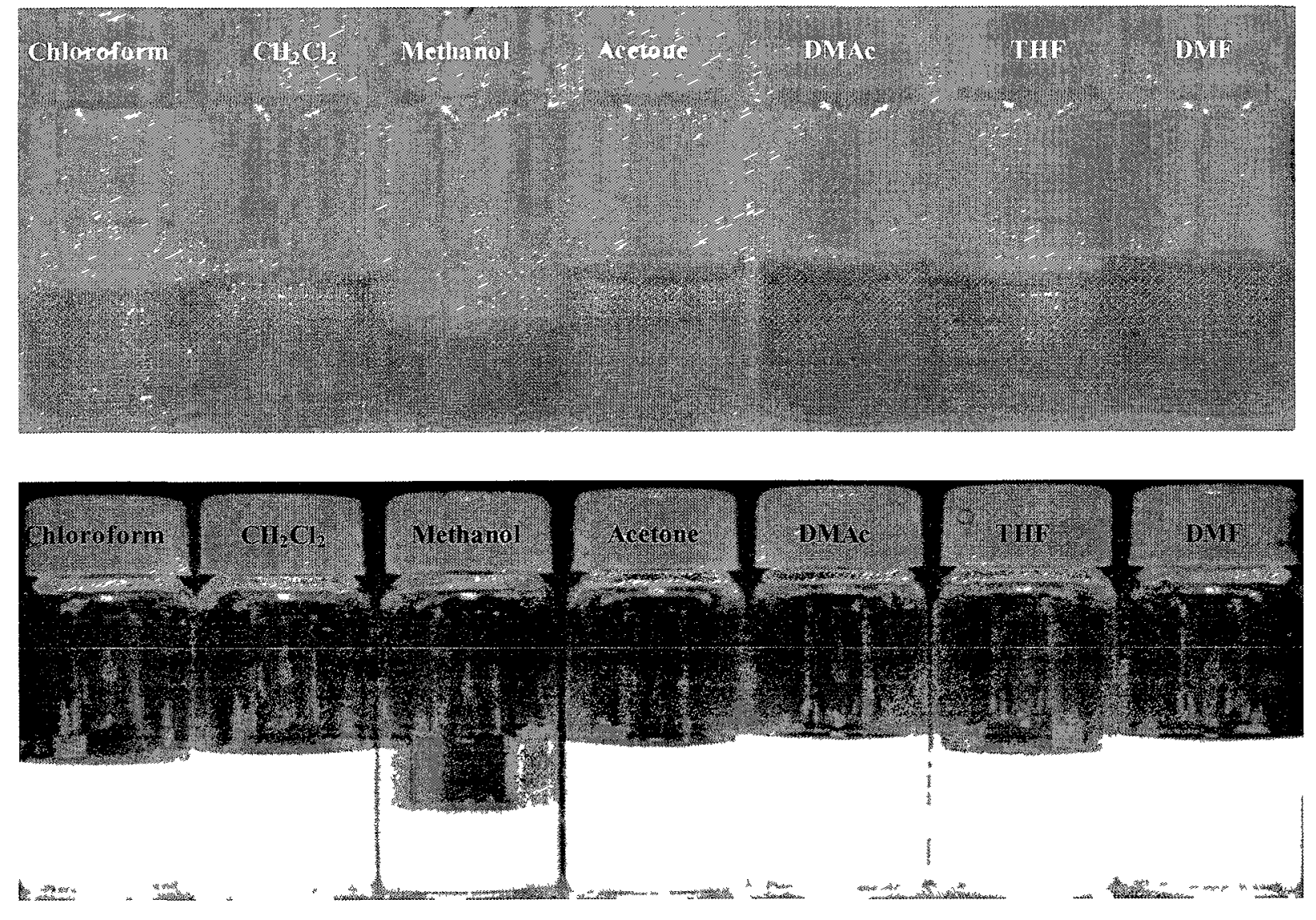

Figure C1: DJPTCDI in different solvents at visible light (upper) and excited at $366 \mathrm{~nm}$ (lower). 

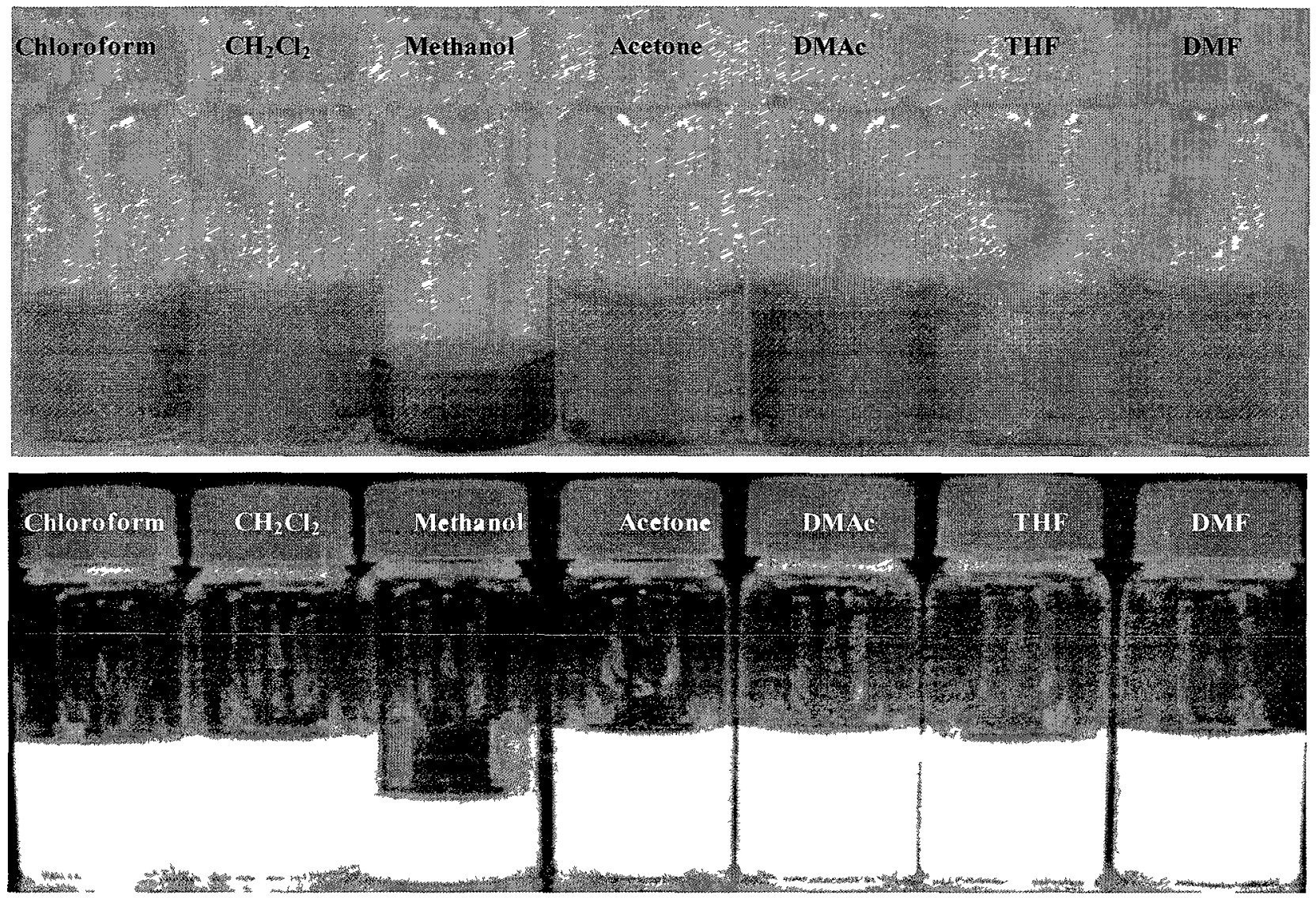

Figure C2: MJPTCDI in different solvents at visible light (upper) and excited at $366 \mathrm{~nm}$ (lower). 


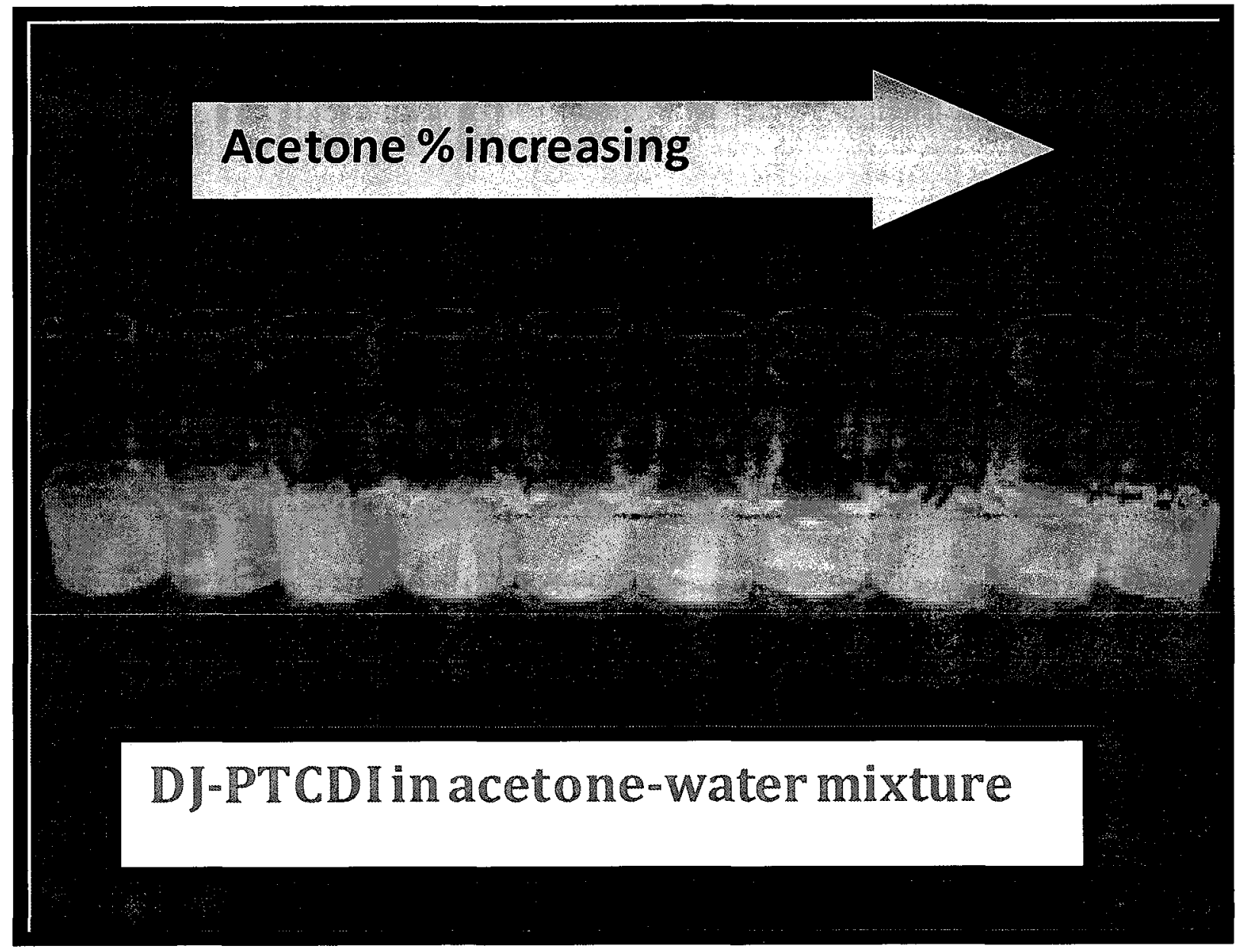

Figure C3: DJPTCDI in different acetone-water mixture excited at $366 \mathrm{~nm}\left(10^{-5} \mathrm{M}\right)$. 


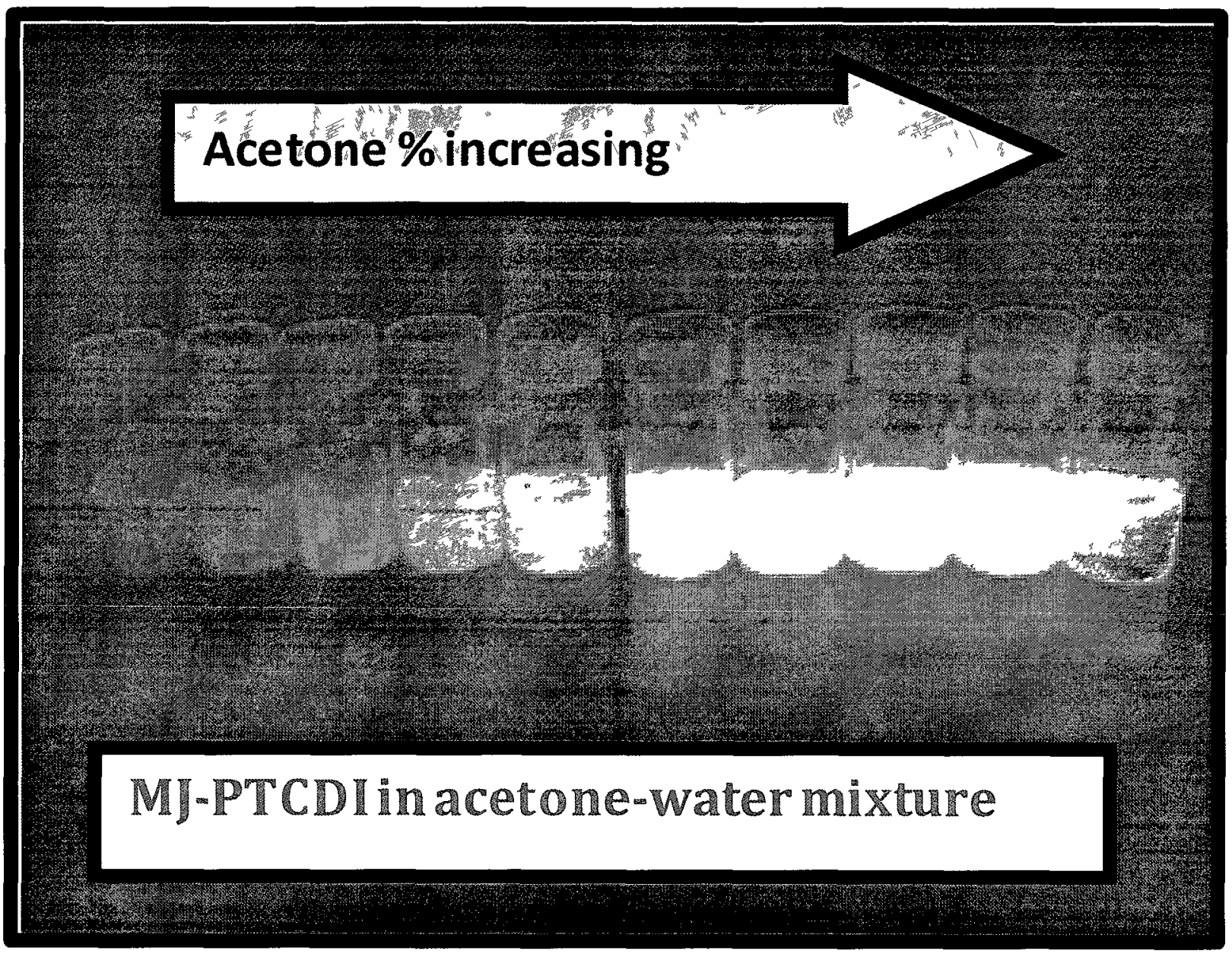

Figure C4: MJPTCDI in acetone-water mixture excited at $366 \mathrm{~nm}\left(10^{-5} \mathrm{M}\right)$. 

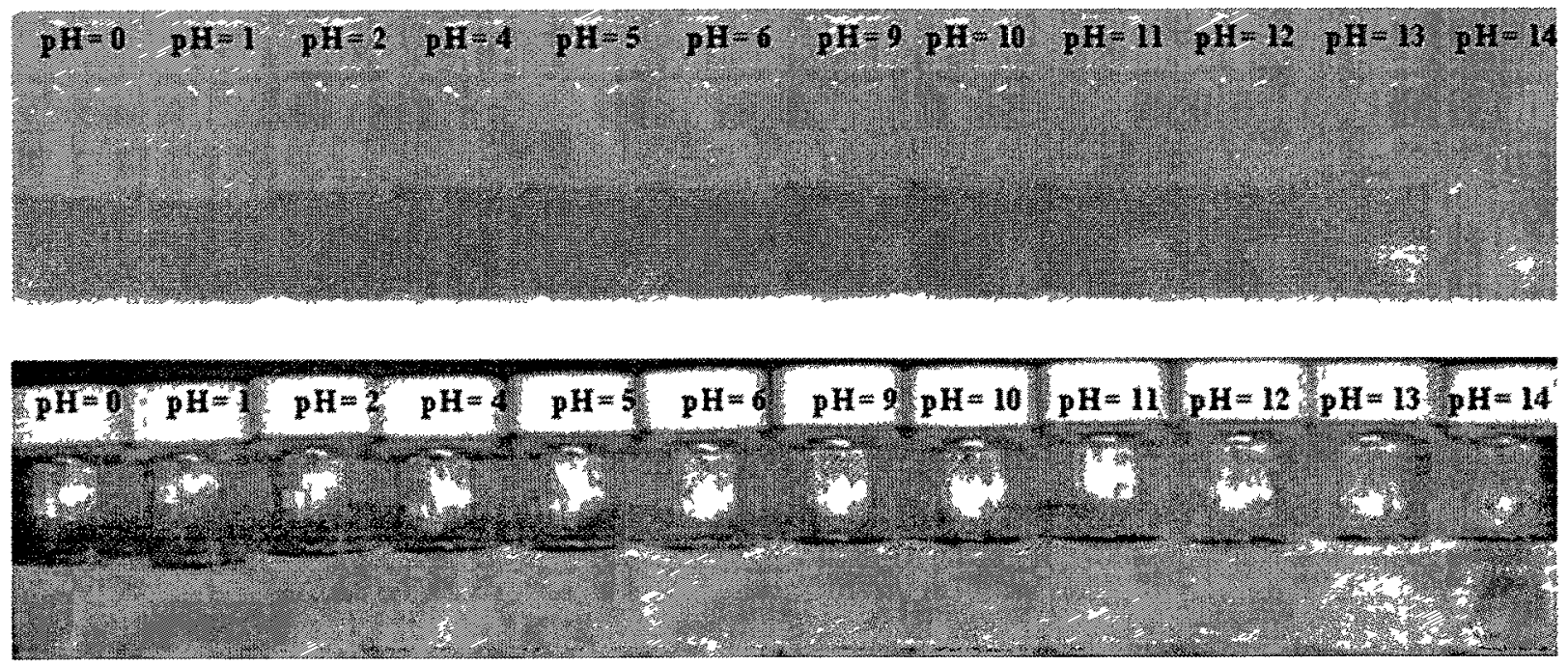

Figure C5: DJPTCDI in different $\mathrm{pH}$ solutions at visible light and excited at $366 \mathrm{~nm}$. 

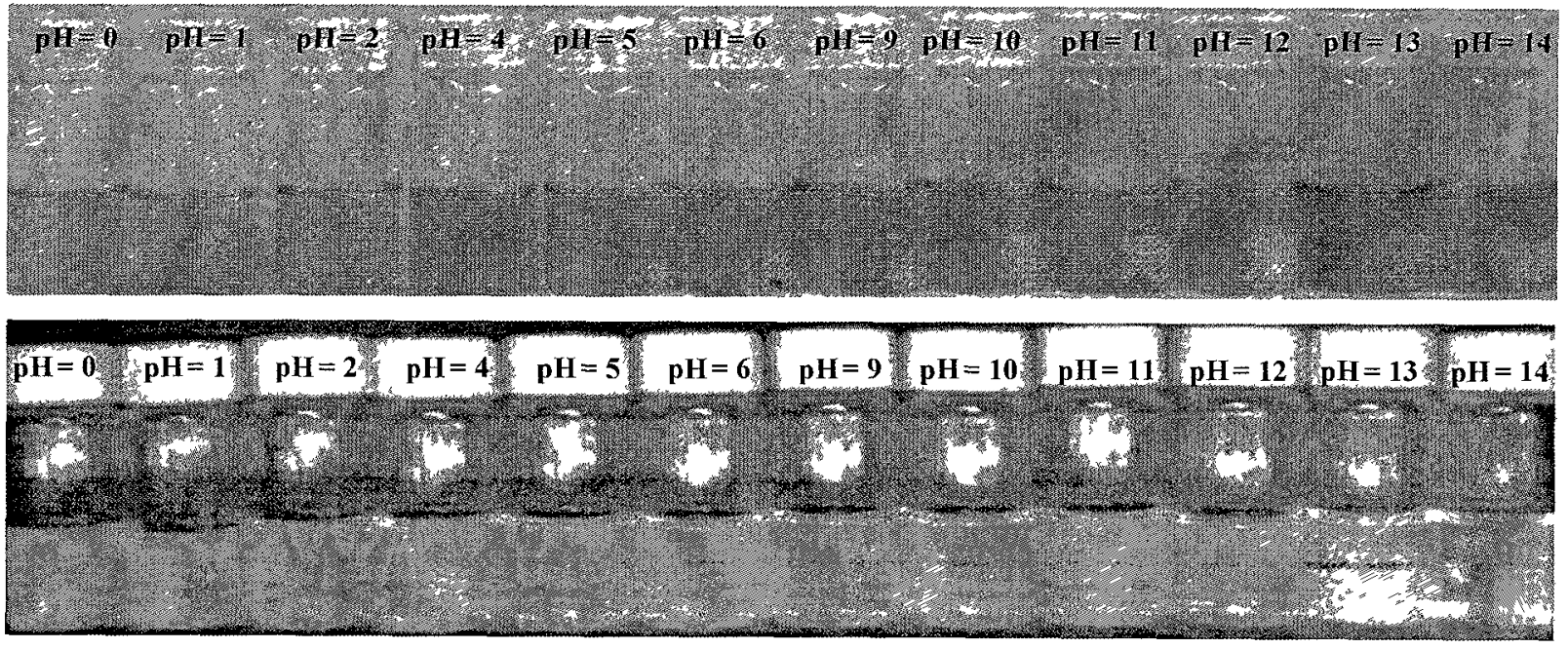

Figure $\mathrm{C}$ 6: MJPTCDI in different $\mathrm{pH}$ solutions at visible light (upper) and excited at $366 \mathrm{~nm}$ (lower). 

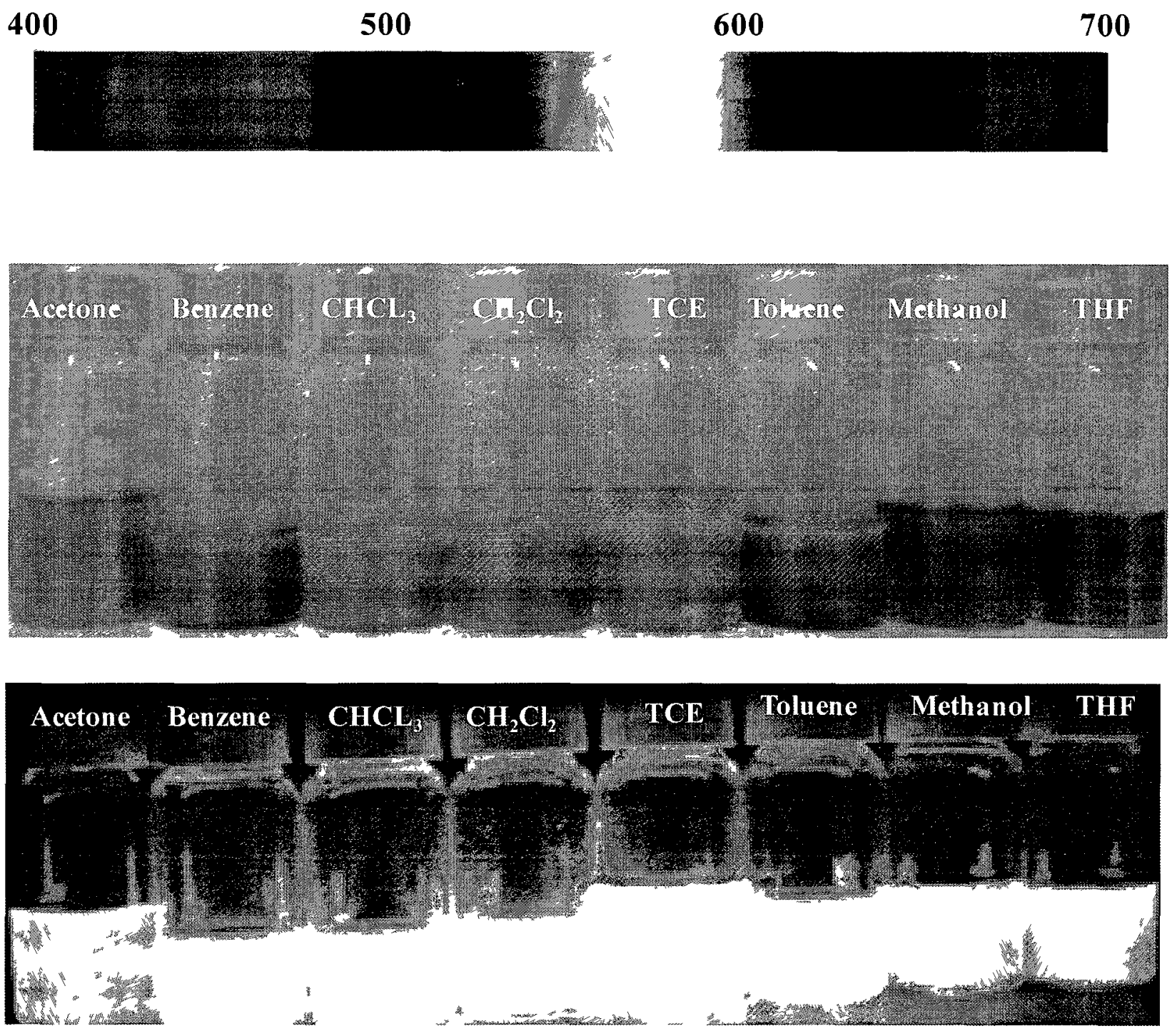

Figure C 7 PJPTCDI in different solvents at 10-4 M exposed to white light (upper) and excited at $366 \mathrm{~nm}$ (lower). 

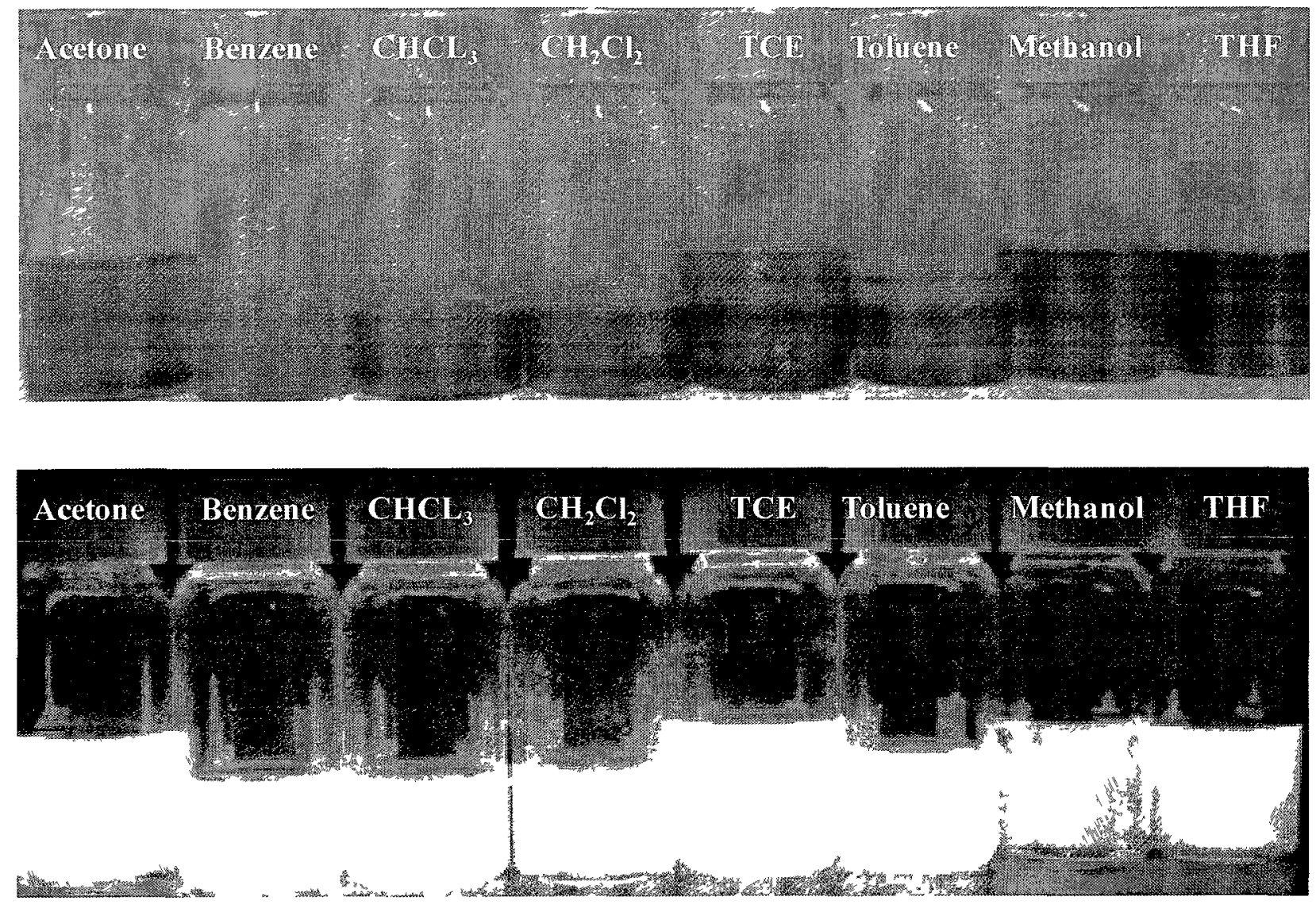

Figure C 8: HJPTCDI in different solvents at 10-4 M exposed to white light (upper) and excited at $366 \mathrm{~nm}$ (lower). 

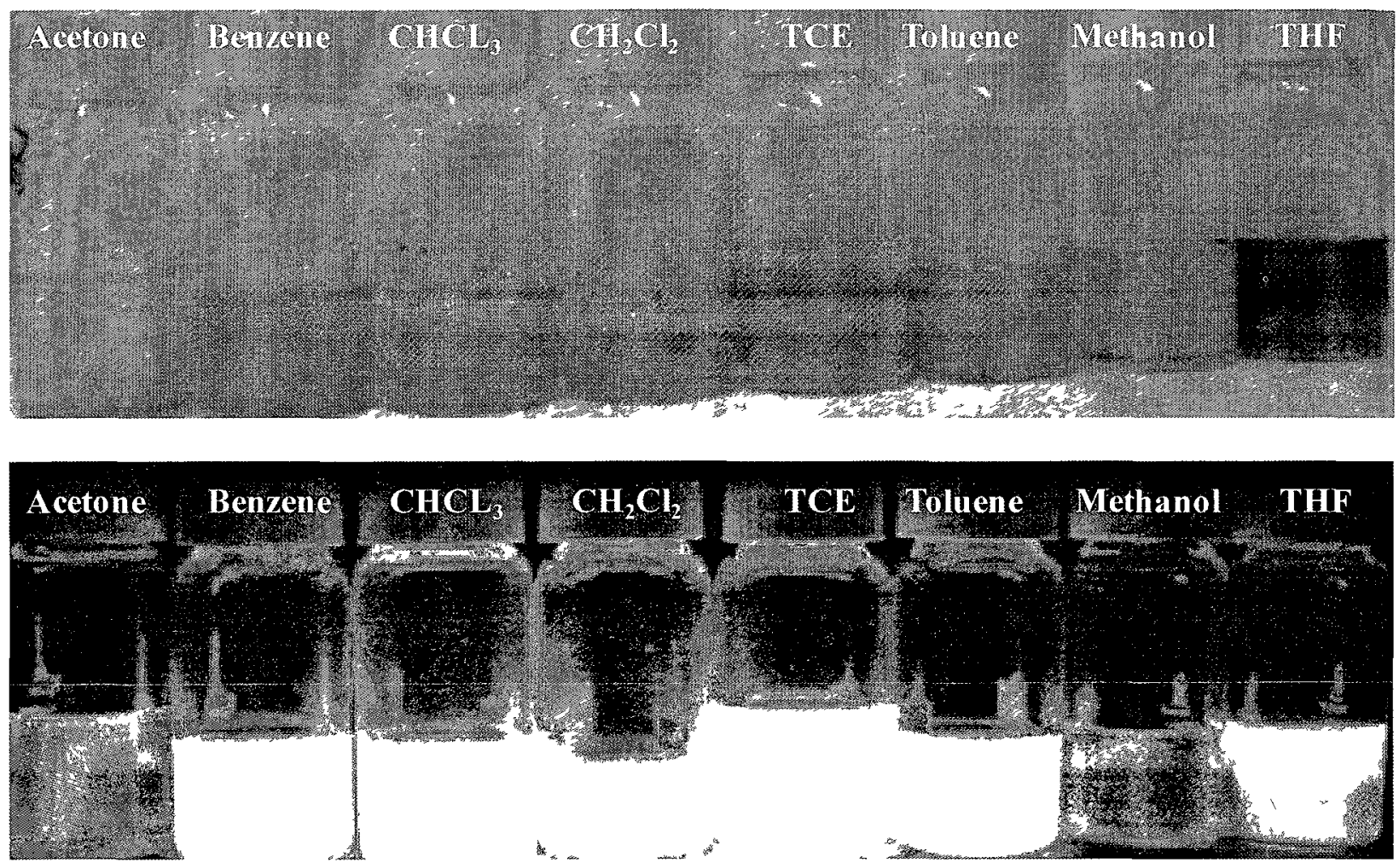

Figure C 9: OJPTCDI in different solvents at 10-4 M exposed to white light (upper) and excited at $366 \mathrm{~nm}$ (lower). 

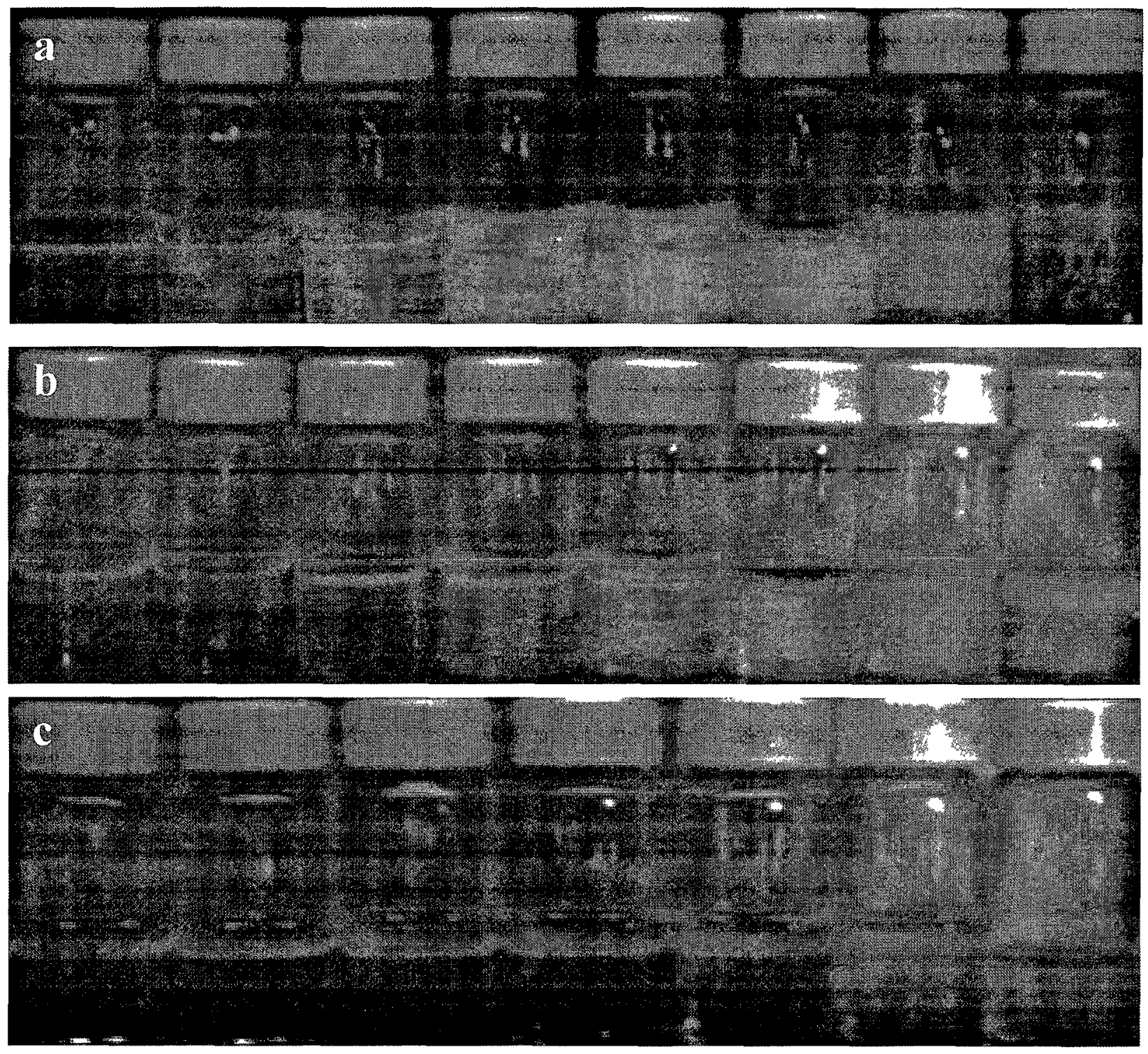

Figure C10: Solution of (a) PJPTCDI, (b) HJPTCDI and (c) OJPTCDI in water at different concentration from $10-7 \mathrm{M}$ to $10-4 \mathrm{M}$ excited at $366 \mathrm{~nm}$. 
Water

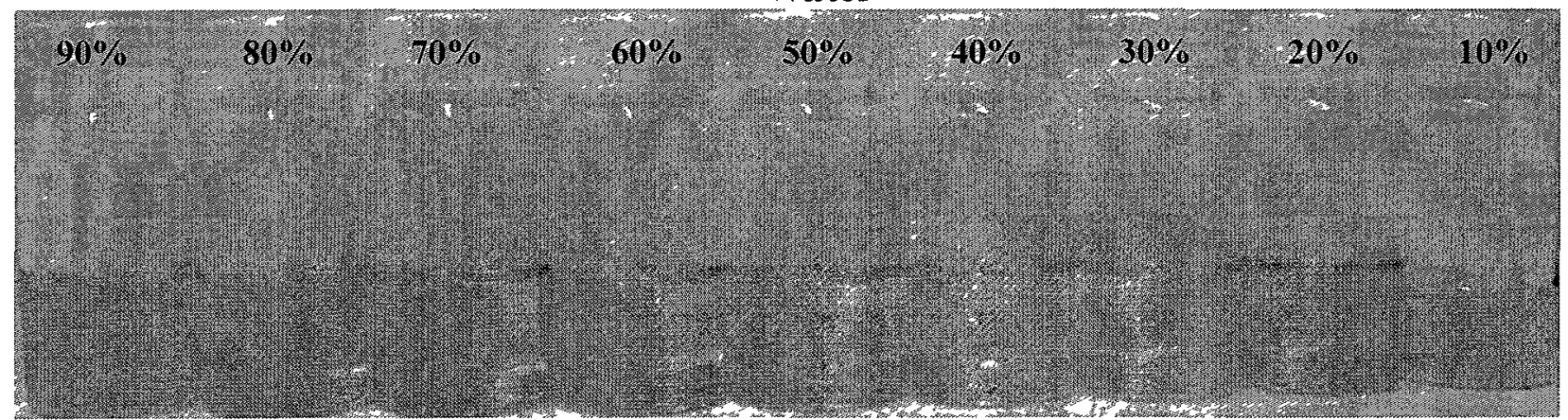

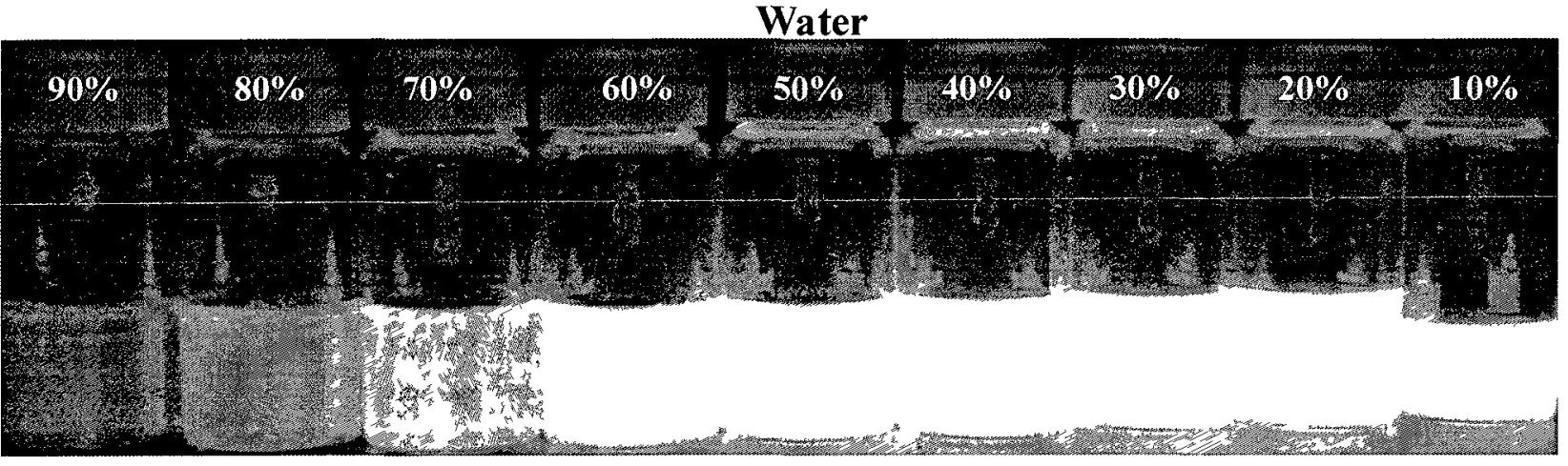

Figure C11: PJPTCDI in acetone-water exposed to white light (upper) and excited at $366 \mathrm{~nm}$ (lower). 
Water

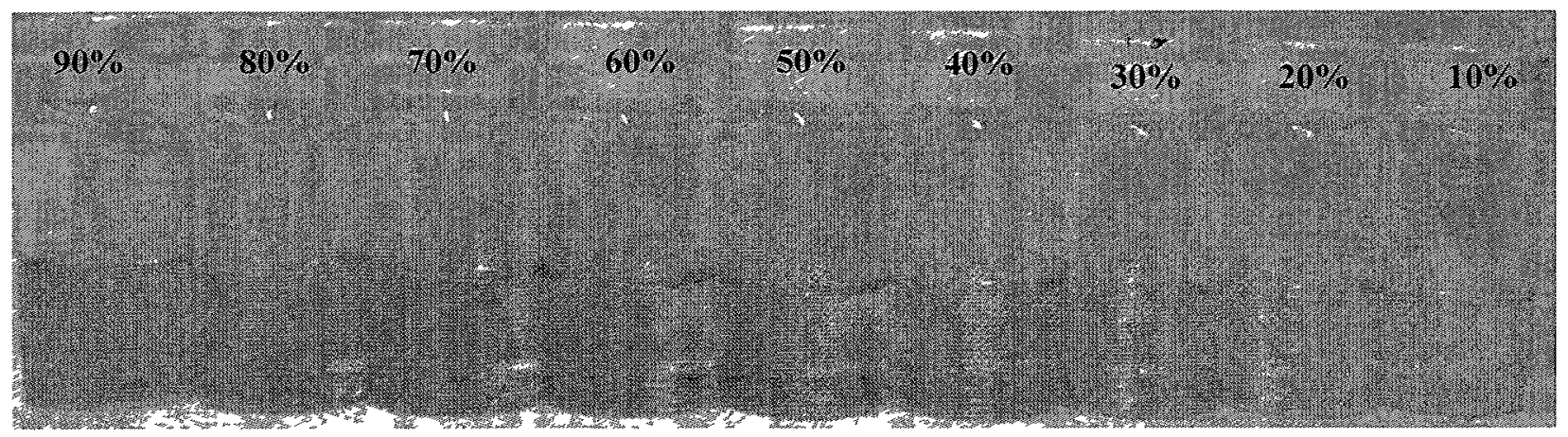

Water

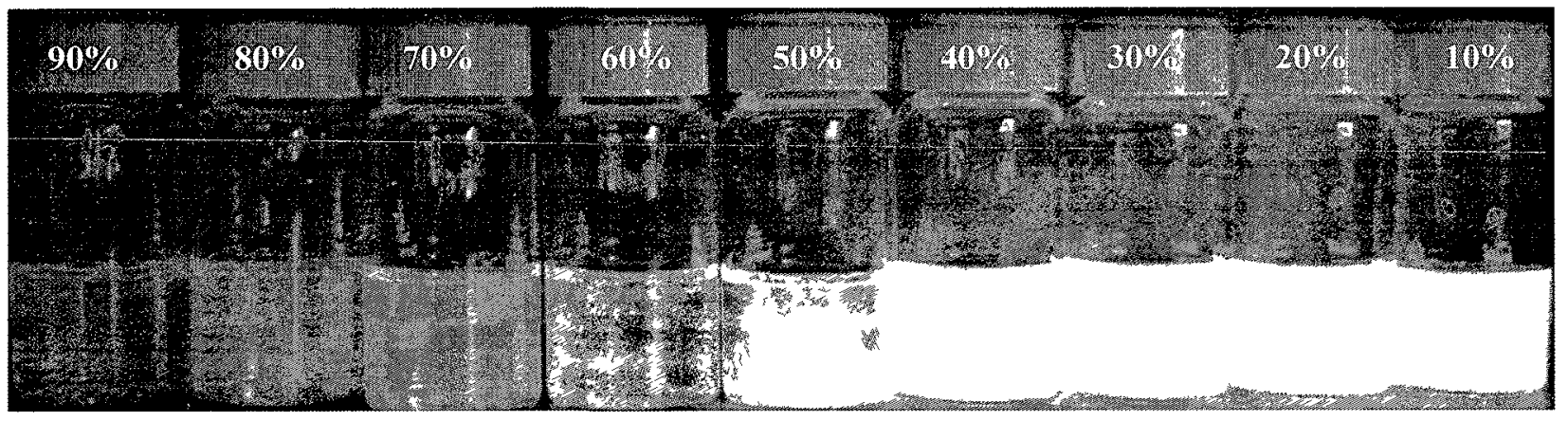

Figure C12: PJPTCDI in methanol-water exposed to white light (upper) and excited at $366 \mathrm{~nm}$ (lower). 